NCCARF

National

Climate Change Adaptation

Research Facility

\title{
以\U Griffith
}

\section{The capacities of private developers in urban climate change adaptation}

Final Report

Heather Shearer, Pazit Taygfeld, Eddo Coiacetto, Jago Dodson and Zsuzsa Banhalmi-Zakar 



\title{
THE CAPACITIES OF PRIVATE DEVELOPERS IN URBAN CLIMATE CHANGE ADAPTATION
}

\author{
DEVELOPING ADAPTIVELY: \\ The role and capacities of private sector \\ developers and financing in urban climate \\ change adaptation: \\ the South East Queensland case \\ Final Report
}

Urban Research Program, Griffith University

AUTHORS

Heather Shearer (Griffith University)

Pazit Taygfeld (Griffith University)

Eddo Coiacetto (Griffith University)

Jago Dodson (Griffith University)

Zsuzsa Banhalmi-Zakar (Griffith University)

NCCARF

National

Climate Change Adaptation Research Facility
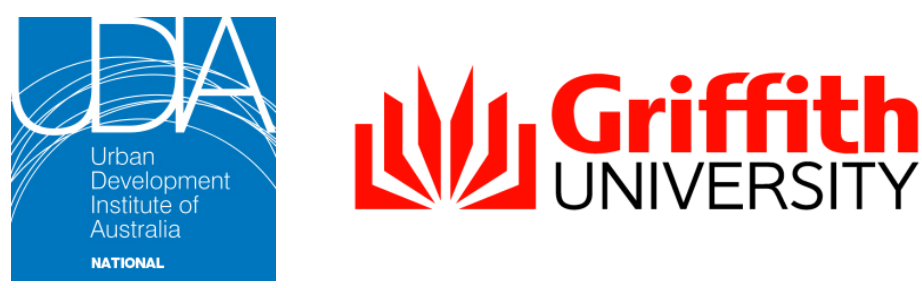
Published by the National Climate Change Adaptation Research Facility 2013

ISBN: 978-1-921609-95-4 NCCARF Publication 24/13

Australian copyright law applies. For permission to reproduce any part of this document, please approach the authors.

\section{Please cite this report as:}

Shearer, H, Taygfeld, P, Coiacetto, E, Dodson, J, Banhalmi-Zakar, Z 2013, The capacities of private developers in urban climate change adaptation, National Climate Change Adaptation Research Facility, Gold Coast, 161 pp.

\section{Acknowledgement}

This work was carried out with financial support from the Australian Government (Department of Climate Change and Energy Efficiency) and the National Climate Change Adaptation Research Facility.

The role of NCCARF is to lead the research community in a national interdisciplinary effort to generate the information needed by decision-makers in government, business and in vulnerable sectors and communities to manage the risk of climate change impacts.

\section{Disclaimer}

The views expressed herein are not necessarily the views of the Commonwealth or NCCARF, and neither the Commonwealth nor NCCARF accept responsibility for information or advice contained herein.

Cover image (c) 2012 Sheba Also 


\section{TABLE OF CONTENTS}

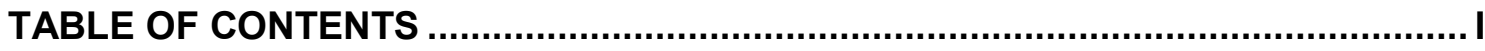

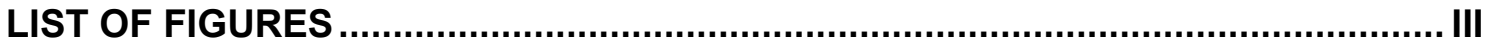

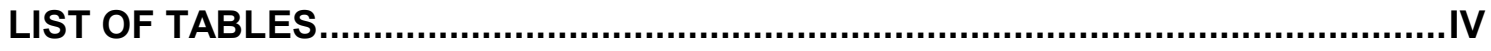

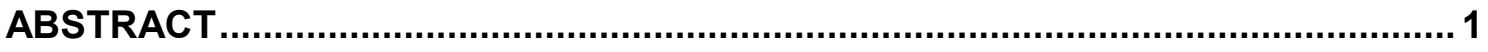

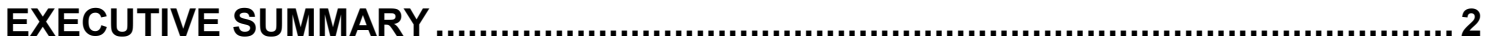

1. OBJECTIVES OF THE RESEARCH.................................................... 5

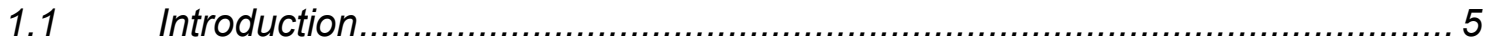

1.1.1 Aim

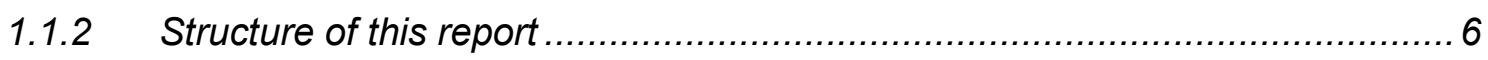

2. RESEARCH ACTIVITIES AND METHODS ................................................ 8

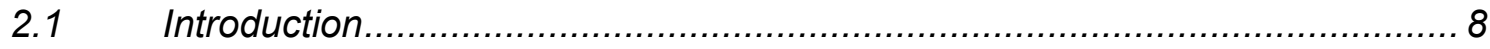

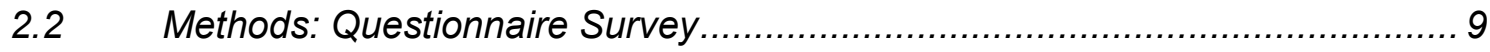

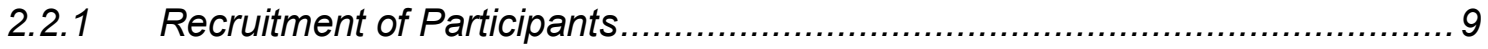

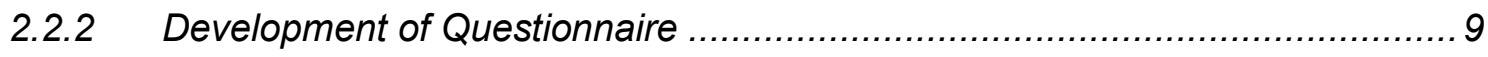

2.2.3 Format of the Questionnaire ............................................................ 9

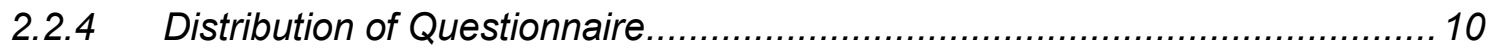

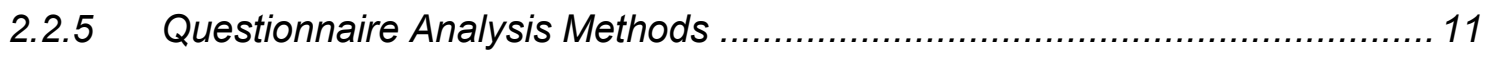

2.3 Methods: Semi Structured Interviews ..................................................... 12

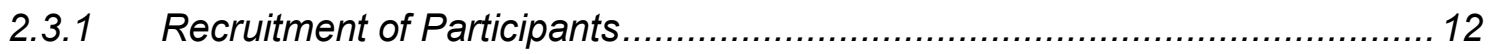

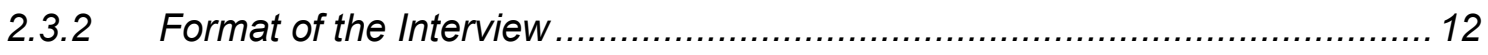

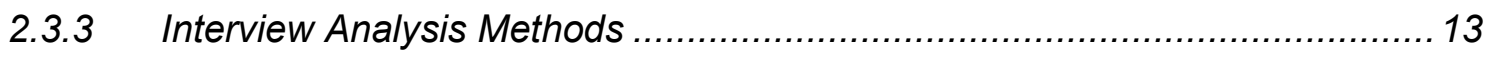

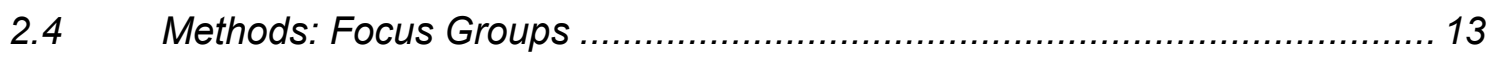

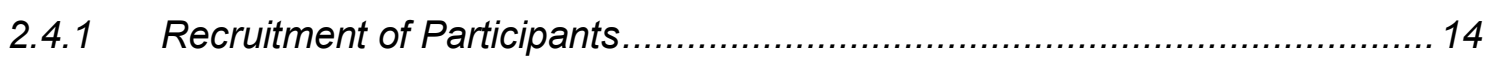

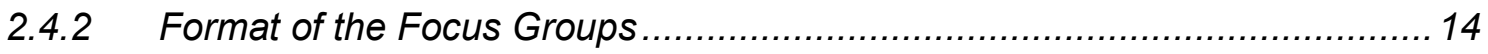

2.4.3 Focus Group and Interview Analysis Methods ...................................... 14

3. CLIMATE CHANGE AND THE PRIVATE URBAN DEVELOPMENT SECTOR -

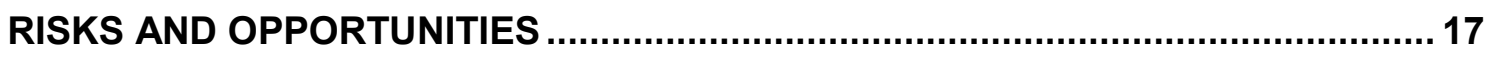

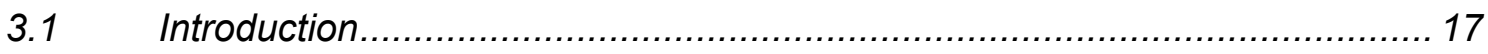

3.2 Climate Change Risks and Opportunities ............................................... 17

3.2.1 Combined Risks - Results from Survey............................................ 18

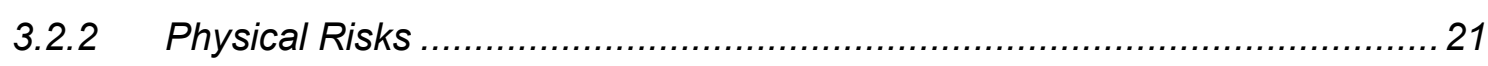

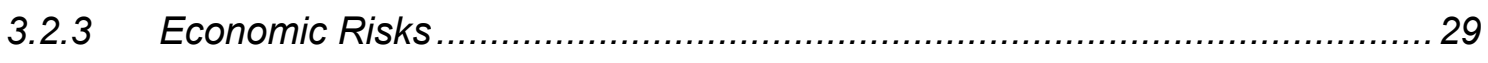

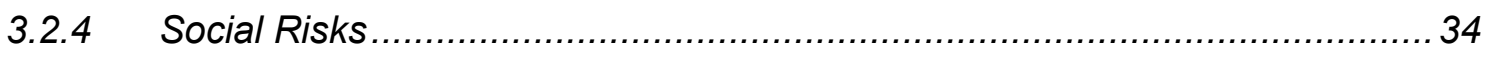

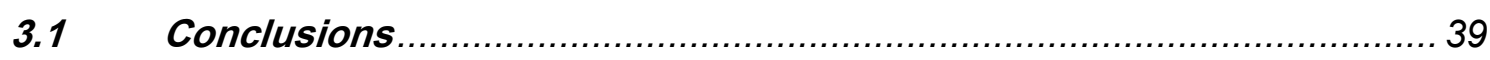




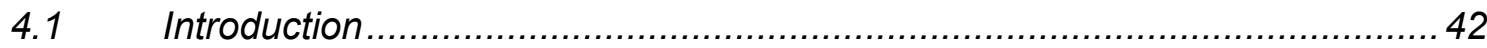

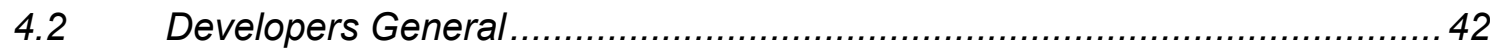

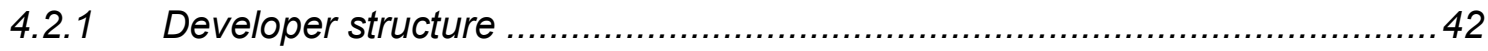

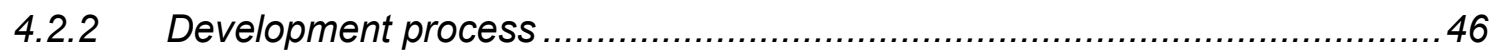

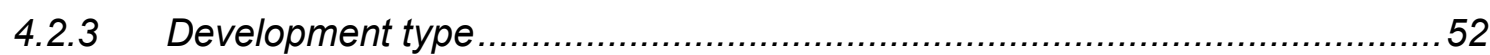

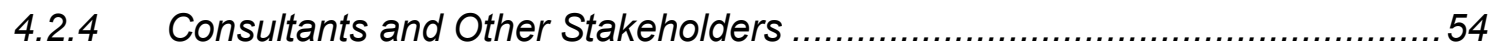

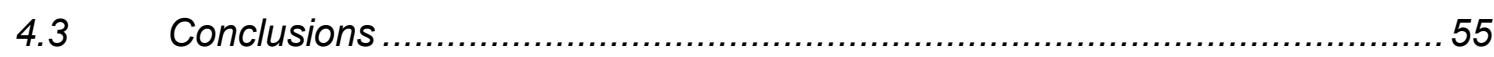

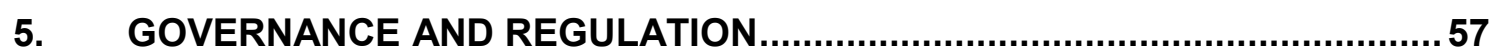

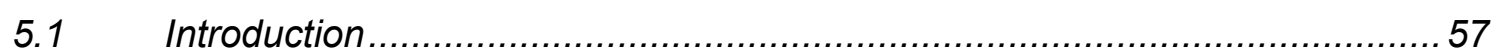

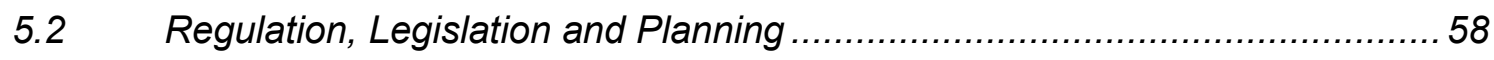

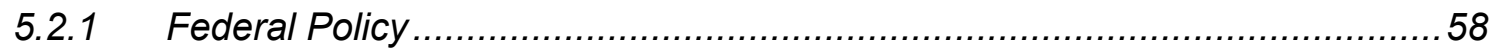

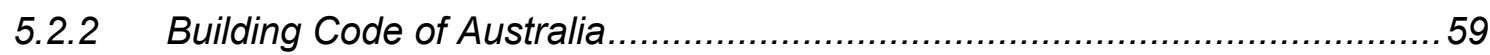

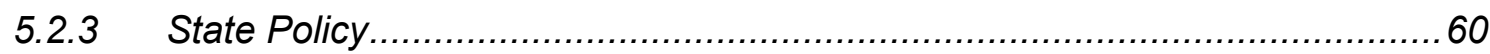

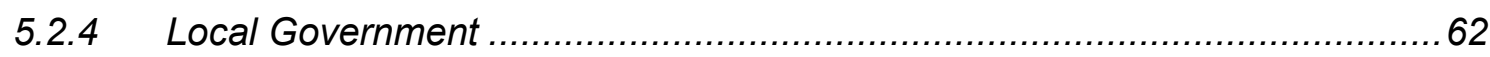

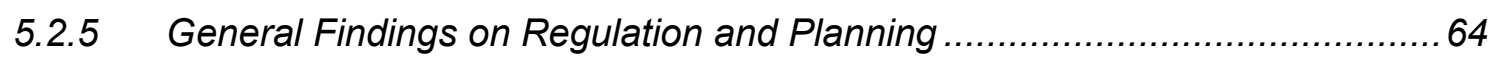

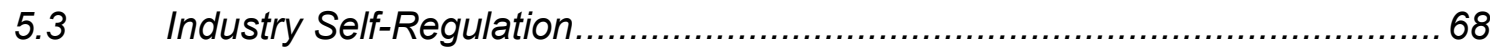

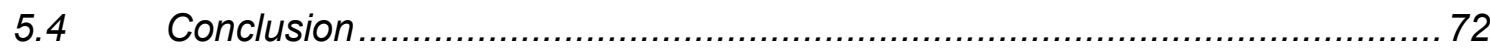

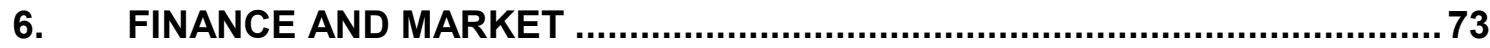

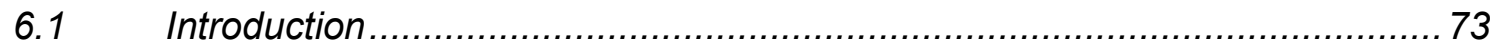

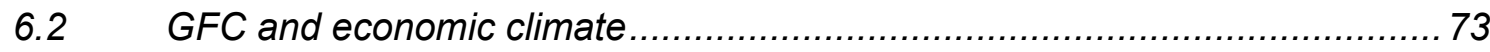

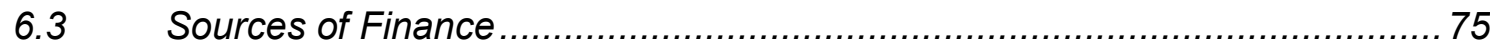

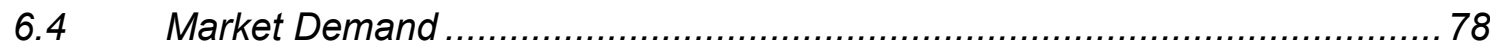

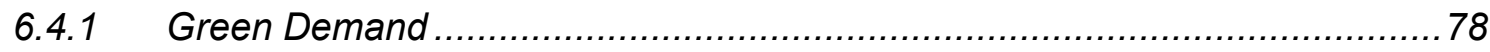

6.4.2 Development Finance and Climate Risk ............................................. 80

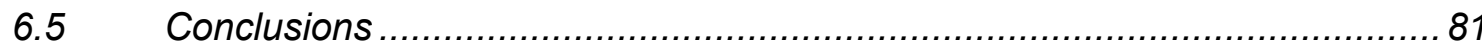

\section{THE ADAPTIVE CAPACITY OF THE PRIVATE URBAN DEVELOPMENT}

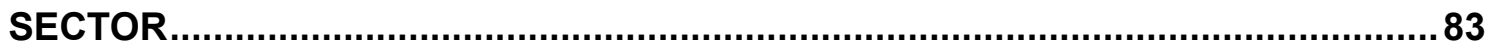

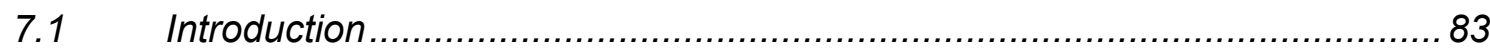

7.2 Developers Response to Climate Change .............................................. 83

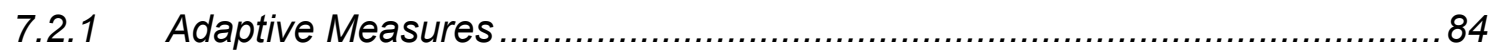

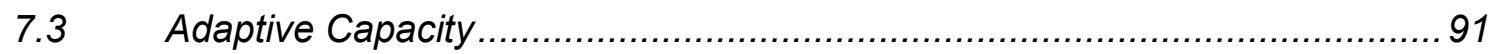

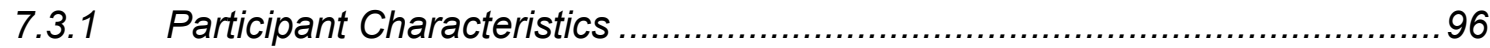

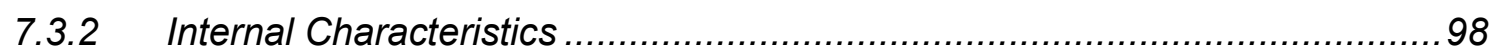

7.3.3 Development Characteristics .............................................................. 102

7.3.4 Economic and Market Characteristics.................................................. 105

7.3.1 Regulation, Legal and Governance Characteristics .............................. 108

ii The capacities of private developers in urban climate change adaptation 


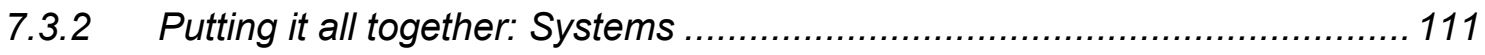

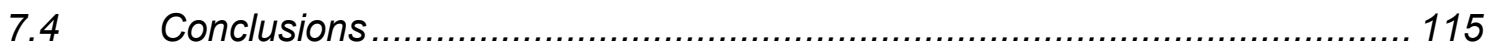

8. CONCLUSIONS, RECOMMENDATIONS AND FUTURE RESEARCH

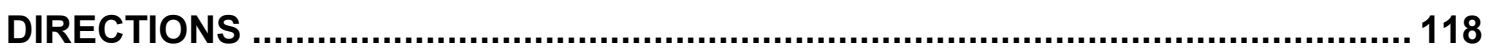

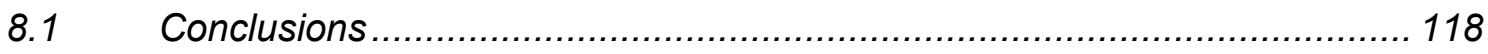

8.2 Knowledge Gaps and Future Research Directions .............................. 121

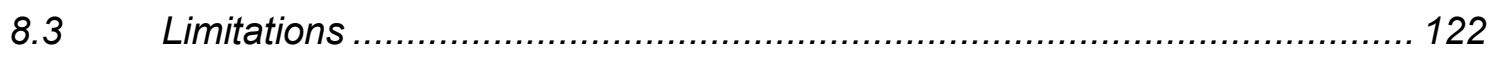

8.4 Recommendations for policy makers ............................................ 123

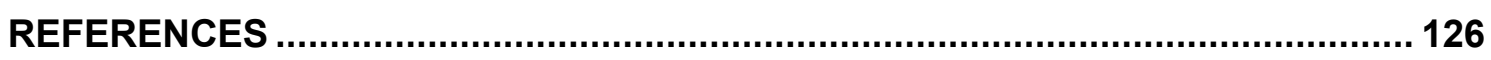

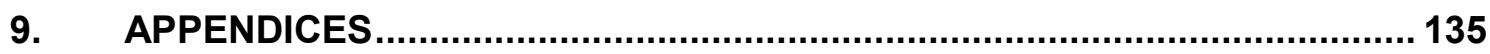

9.1 Information Sheet and Consent Form for Interview and Focus Group

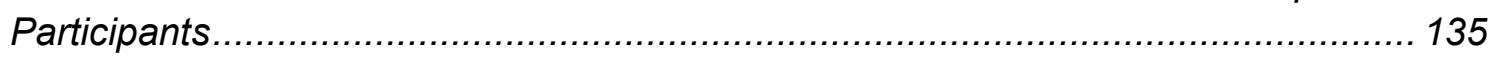

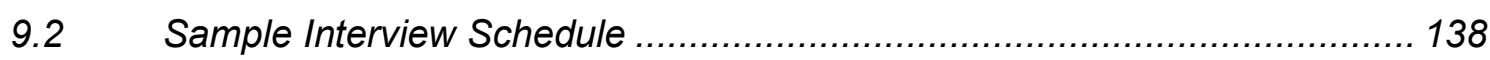

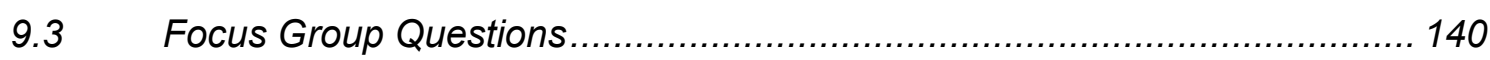

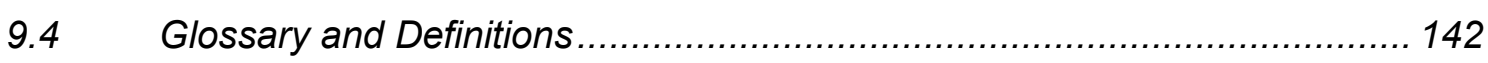

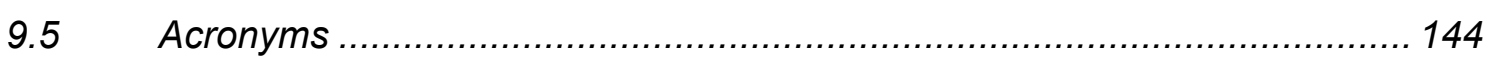

9.6 Coding for Interviews and Focus Groups.......................................... 146

9.7 Survey Questions ....................................................................... 147

\section{LIST OF FIGURES}

Figure 1 Schematic of Research Methodology......

Figure 2 Potential Impact of the Physical Hazards of Climate Change (by respondent type)

Figure 3 Storm Surge Damage from Cyclone Yasi, Townsville (Source: Rob and Stephanie Levy (2011) via Wikimedia Commons)

Figure 4 Infrastructure Damage after Cyclone Larry, Mission Beach, 2006 (Source:

http://www.rainforest-facts.com/ via Wikimedia Commons).....

Figure 5 The "Black Saturday" Bushfires, Victoria (2009) (Source: Nick Carson (2009) via Wikimedia Commons) .....

Figure 6. Percentage Losses from Natural Catastrophes (Adapted from Munich $\mathrm{Re}$ 2012)

Figure 7. Rise in North Queensland Strata insurance premiums since 2007 (Adapted from Australian Government Actuary, 2012) ....

Figure 8 Number and Type of Firms Participating in Research Project (Survey, Interviews and Focus Groups)

Figure 9. Stages in the property development process (Adapted from Birrell and Gao, 1997) 
Figure 10 Type of Development Product (Survey Respondents).

Figure 11 References to the Australian Building Code (and building codes in general)

Figure 12 Ranking of Survey Respondent Perceived Importance of Regulatory Issues in Comparison with all Secondary Issues

Figure 13 Percentage of Survey Respondents using Industry Self-Regulation Tools (note: respondents could select multiple choices) ....

Figure 14 EnviroDevelopment Logo showing Leaf Symbols (Source:

http://www.udiawa.com.au/go/industry-envirodevelopment) ..................................70

Figure 15 Sources of Funding for Survey Respondents ........................................ 77

Figure 16 Adaptive Measures (Survey Respondents) ........................................... 86

Figure 17 Survey Respondents Opinions on General Adaptability Issues ...................94 94

Figure 18 Potential Drivers for Adaptive Capacity (Survey Respondents) (green =

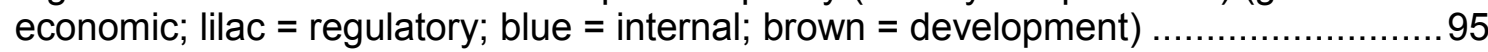

Figure 19 Potential barriers to climate change adaptation (survey respondents).........96

Figure 20 Survey Respondents Self Rating of Climate Change Knowledge ................99

Figure 21 The concepts of leadership, legacy and brand .................................... 101

Figure 22 Coding References to Time............................................................ 104

Figure 23 Schematic of Measures of Adaptive Capacity ..................................... 114

\section{LIST OF TABLES}

Table 1 Final Themes (Nodes) and Sub Themes Identified in Qualitative Analysis ..... 16

Table 2 Ranked and Categorised Risks from Survey Respondents .20

Table 3 Largest Catastrophe Events in Australia (2011 adjusted \$ amount) Adapted from Insurance Council of Australia (ICA)

Table 4 Proportion of developers working in various regions of South East Queensland (Survey Respondents only).

Table 5 Changes in key lending criteria post GFC (Adapted from Bryant 2012 and Ashe

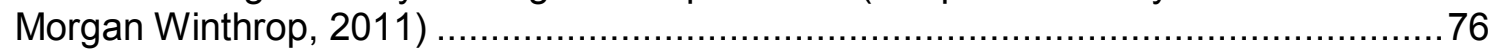

Table 6 Examples of Adaptive Measures (adapted from Sovacool, 2011; Smit \& Pilifosova 2003 and Burton, 1996) ....

Table 7 Does your firm incorporate adaptation to future climate change into decision making or current operations?

Table 8 General Measures of Adaptive Capacity (expanded from classifications of (Smit \& Pilifosova, 2003) and (Burton, 1996).

Table 9. Key findings and associated recommendations 125 


\section{ABSTRACT}

The urban environment is particularly exposed to climate impacts, due to a range of factors, such as its geographical location, spatial immobility, and high population. The urban development industry relates to climate change because the built environment itself has climate impacts; the location and scale of development products influence the degree to which users are exposed to climate hazards; and climate change presents multiple challenges to which developers will likely need to adapt. However, a major policy and knowledge gap exists in the social scientific understanding of the private sector developers' capability to respond in an effective and timely way to the climate adaptation challenge. Using South East Queensland (SEQ) as a case study, this research investigated the institutional capacity of the private urban development sector to respond to climate change. This report explores findings from an online questionnaire survey, and a series of interviews and focus groups.

In general, the adaptive capacity of SEQ development industry is dependent on a combination of complex and interconnected factors, such as: financing and market conditions; government regulation and industry self-regulation; firm size and structure; the type and spatiotemporal scale of developments; and the concepts of leadership and legacy. These factors could be broadly characterised under the following categories; participant characteristics, internal attributes, development characteristics, regulation, and economic and market characteristics.

Development firms with a greater adaptive capacity were generally larger, with greater economic resources, and were less reliant on bank funding. They also had a diverse range of development products, and could afford to hire or contract specialist consultants. Commercial developers were also more likely to incorporate adaptive measures into developments than were residential developers. This was driven by tenant demand, government rebates, and reduced operating and maintenance costs. Commercial developers also subscribed to industry certification and rating schemes such as Green Star and EnviroDevelopment. On the other hand, residential developers sold rather than leased development products; and reported little, if any demand for sustainable features from the market, which was primarily price driven.

The major barriers to adaptive capacity were economic, marketing and regulatory issues. The development economy had not fully recovered from the Global Financial Crisis (GFC). The GFC had also severely curtailed bank lending for property development; financiers required a higher percentage of equity and presales, and required loans to be repaid in less than two years. State and Local Government regulation and processes were also seen as barriers to adaptation; regulation was viewed as reactive, complex, inconsistent and inflexible. The perceived slowness of local government was a particular problem.

Generally speaking, the SEQ development industry had low adaptive capacity, but this also depended on the financial, insurance and government players. The insurance and financial sectors had the potential to drive adaptation, but current practices resulted more often in maladaptation. Further research into the role of allied professionals, such as consultants and contractors, would be valuable; as would research into the potential for the insurance industry to drive climate change adaptation. 


\section{EXECUTIVE SUMMARY}

The urban environment is particularly exposed to climate change impacts due to a range of geographic and social factors. For example, cities are often located on floodplains or near the ocean; areas that are vulnerable to floods and sea level rise. Cities are also dependent on the import of resources from external regions, which may be exposed to additional impacts, such as drought, bushfire or social unrest. However, the built environment is spatially fixed; and urban infrastructure, such as buildings, roads and rail, cannot easily or cost effectively be relocated to less vulnerable locations.

Urban development relates to climate change in at least three important ways. First, the product of development-the built environment-has climate impacts through the materials used; and the design and location of developments. Second, the nature of the developer's products and the resulting built environments-such as its location and scale-influence the degree to which users are exposed to climate change hazards. Third, climate change presents direct and indirect challenges and hazards to which developers will need to adapt.

The future environments built through property development activity will strongly shape future urban capacity to respond and adapt to climate change impacts. The current and future development decisions and the flows of investment that follow from these decisions have the potential to significantly influence the national capacity to adapt to climate change. Therefore, improving the knowledge of the capacity and weaknesses of private urban development and financing institutions in the area of climate change is a critical imperative within a wider policy of responsive climate change adaptation. Ignoring the private development sector and its institutional complexities would be to ignore a major potential source of improved adaptive activity.

This study aims to investigate the institutional capacity of the private urban development sector in South East Queensland (SEQ), Australia, to respond to the task of climate change adaptation and in turn, investigate the role of private financial institutions in funding climate adaptive urban development. To do so, it used a desktop literature review, an online questionnaire survey, and a series of semi-structured interviews and focus groups with developers, consultants, State and Local Government staff, architects, solicitors, planners and financiers.

A total of 62 people responded to the online survey; and 21 interviews and 3 focus groups (9 participants) were held. Developer firms were diverse, ranging from large ASX-listed companies to small family firms; these also included two developers representing quasi-government corporations. Likewise, consultancies were widely diverse, ranging from multinational engineering firms to one person practices; two of which also specialised in climate change adaptation. Two financiers, two architects, a solicitor, and State and Local Government representatives were also interviewed.

The results of this research demonstrated that the adaptive capacity of the urban property development industry is dependent on a combination of complex and interconnected factors. The most important factors determining adaptive capacity were economic resources, financing and market conditions; government regulation and industry self-regulation; firm size and structure; the type and spatiotemporal scale of developments; and the concepts of leadership and legacy. These factors could be broadly characterised under the following categories; participant characteristics, internal attributes, development characteristics, regulation, and economic and market characteristics. 
The size and company structure of developers was significant. Larger developers with a range of diverse products had more flexibility to introduce adaptive measures, as they were less dependent on bank funding, and could also afford to employ or contract specialist consultants. Such firms were also conscious of their enduring legacy, and intended their development products to showcase their brand. However, some smaller developers were also driven to produce high quality products, and this motivation largely depended on personal attitude.

Economic conditions and finance were highly significant. In the post Global Financial Crisis (GFC) environment, market conditions and the availability of finance largely dictated the type of development produced, particularly for smaller firms. Previously, SEQ developers had profited from the region's high population growth. However, after the GFC, the demand for property, particularly units, had plummeted. The GFC had had a major impact on the development industry, with many firms going out of business, or moving to other ventures.

The GFC also negatively impacted on the financial sector. During the post GFC period, financiers began to impose a range of increasingly onerous conditions on development loans, requiring a high percentage of pre-sales (often greater than $75 \%$ ), more stringent reporting requirements, and a higher proportion of equity. Thus, all respondents reported difficulty in obtaining bank finance for developments; with some developers seeking alternative funding sources, such as high net worth individuals, or even international private equity.

The economic situation also influenced how developers marketed their products. Pre GFC, many firms had used "green" sustainability features to differentiate their products from their many competitors. However, post GFC, purchasers were significantly more price conscious, with the result that the "nice to have green measures" were omitted.

However, commercial developers continued to incorporate sustainability features into buildings, in response to tenant demand, reduced operating costs and government subsidies. There was little demand for sustainability features from the residential market, unless these incurred no additional cost. To a limited extent, the retirement sector wanted some sustainability features; this was driven by the desire to reduce costs, particularly energy costs. Thus, market demand served as both a driver and a barrier to adaptive capacity.

The time, scale and type of development were also major determinants of adaptive capacity. In general, the larger the development, and the longer the development time frames, the more likely it was that developers were concerned about the potential risks of climate change, and would implement adaptive measures. The converse was true for smaller developments with shorter time frames. For these, the developer's main goal was to complete the development as quickly as possible, in order to reimburse bank loans. Lending policies exacerbated this, as banks were not prepared to loan money to developers for longer than a maximum of two years.

The regulatory environment was generally seen as a barrier to adaptive capacity, with a plethora of complex, confusing and inflexible regulation from all levels of government. However, the Building Code of Australia (BCA) was viewed by many as the regulatory avenue with the most potential for compelling climate change adaptation. For example, past climate events, such as Darwin's Cyclone Tracey (1974) had resulted in rapid changes to the BCA, to which all developers have to adhere.

Other potentially important avenues for adaptation were attitudes and knowledge. A number of respondents expressed confusion and scepticism regarding climate change, and the potential impacts. They associated climate change primarily with sea level rise, and saw this happening, if at all, in the distant future, and thus, posing minimal risk. Despite this, many commented on the need to incorporate environmentally sustainable features into developments, particularly if there was a market demand for them. 
Developers also regularly used industry-specific certification schemes such as Green Star and EnviroDevelopment. To some extent, the development industry appears willing to adapt to climate change, but the risks need to be better communicated.

Further, the insurance industry has the potential to drive adaptive capacity. In North Queensland, extreme weather events, such as Cyclone Yasi (2011) had resulted in large premium rises, particularly for strata titled units. Developers reported great difficulty in selling these properties, as the Body Corporate fees were extremely high due to the insurance costs. However, unless insurers link premiums to the introduction of adaptive measures, price rises are likely to be maladaptive, resulting in properties with inadequate or no insurance.

While all of the above factors could be drivers or barriers to adaptation, combining these into a rating scale of adaptive capacity would be unproductive. These factors are all interconnected, and a high "score" on one factor, may mean a low "score" on another. Moreover, adaptive capacity is not fixed; it is flexible, dynamic, multidimensional and dependent on a number of interrelated factors.

Therefore, one potentially useful way of capturing adaptive capacity is to use a systems approach; by focussing on how the firm and its development products interact with the broader social, economic, environmental and political environment in which it operates (Smith et al., 2008). In this manner, the property development industry is viewed as a dynamic player in a complex environment, which can and does change in response to the interaction of multiple, and interrelated factors.

In conclusion, this research has identified some important drivers of adaptive capacity for the urban property development industry: such as the size and structure of a developer; industry self-regulation schemes; the insurance industry; and the flexibility of the BCA to incorporate adaptive measures. Other potentially important drivers could include encouraging demand for sustainable features in the residential market; perhaps by quantifying long term savings or subsidising developers, as in the commercial sector. Further, if financiers could tie loan conditions and/or the insurance industry could link premiums to the provision of adaptive measures; this could serve as a major driver. Finally, the desire by developers to be recognised as professionals, in combination with brand and legacy, are also important.

More research is needed on many of these, particularly on the potential for the financial and insurance sectors to drive adaptation. At present, both these industries are serving more as agents of maladaptation; with financiers putting time pressure on developers, and insurers raising premiums without requiring adaptive changes. 


\section{OBJECTIVES OF THE RESEARCH}

\subsection{Introduction}

Urban environments are particularly exposed to climate change because of various social and structural reasons (Bambrick, Capon, Barnett, Beaty, \& Burton, 2011). Urban areas are often located on floodplains or near the ocean; areas which are vulnerable to floods, sea level rise and other impacts of climate change. However, the built environment is spatially fixed. Infrastructure such as buildings, roads and rail cannot easily or cost effectively be relocated.

In addition, a large proportion of the world's population lives in urban areas, and cities around the globe are rapidly growing from in-migration. This concentration of large populations exposes greater numbers of people to climate impacts (Bambrick et al., 2011). Further, cities are also dependent on the import of essential resources, such as food and water; extending their vulnerability to other regions, which may be subject to additional impacts, such as drought or bushfire.

The future impacts of climate change are likely to become more intense and potentially destructive (CSIRO, 2011). The current scientific consensus is that a $2^{\circ} \mathrm{C}$ warming is unavoidable (New et al. 2011). Moreover, it is increasingly likely that by the end of the 21st century, average global temperatures will increase by approximately $4^{\circ} \mathrm{C}$ (Meinshausen et al., 2009; Sanderson, Hemming, \& Betts, 2011; M. Stafford Smith, Horrocks, Harvey, \& Hamilton, 2011; World Bank, 2012b, p. 16).

Human society (comprising governments, firms and individuals) has two major options to respond to climate change: it can choose mitigation (implementing measures, such as carbon pricing) to reduce GHG emissions; or adaptation (changing behaviour or ways of doing things to lessen the impacts when they occur) (Smit \& Wandel, 2006).

Mitigation attempts to limit global climate change by reducing total emissions or concentration of GHGs; enhancing GHG sinks (i.e. planting trees); or changes to land use planning policies, particularly those associated with urban density, transport provision and energy (Gleeson \& Spiller, 2010; Melia \& Barton, 2011).

However, global efforts to curb emissions have to date, had relatively minor impact on GHG levels. Therefore, the world should prepare to live with the impacts associated with a dramatic increase in heatwaves, intense droughts, major floods, and other severe weather events (World Bank, 2012b, p. 16). In other words, the world needs to engage in efforts to adapt to climate change.

The term climate change adaptation has a range of different and often contradictory definitions, according to a specific discipline or policy focus (Australian Government Productivity Commission, 2011; Bosello, Carraro, \& De Cian, 2009; Smit, Burton, Klein, \& Wandel, 2000). In the context of this report, climate change adaptation is defined as:

\section{"A process by which strategies to moderate, cope with and take advantage of the consequences of climatic events are enhanced, developed, and implemented." (United Nations Development Program (UNDP), 2012)}

Therefore, climate change adaptation refers to a process whereby a range of strategies and measures are employed in an attempt to reduce the vulnerability in a system (i.e. a household, community or firm) to climate change; or to capture the opportunities presented by climate change (Smit \& Wandel, 2006).

On the other hand, it is important that adaptive measures do not inadvertently give rise to maladaptation; a process which may be defined as: 


\begin{abstract}
"Action taken ostensibly to avoid or reduce vulnerability to climate change that impacts adversely on, or increases the vulnerability of other systems, sectors or social groups" (Barnett \& O'Neill, 2010).
\end{abstract}

The future environments built through property development activity will strongly shape future urban capacity to respond and adapt to climate change impacts. How current and future development decisions are made and the flows of investment that follow from these decisions have the potential to significantly influence the national capacity to adapt to climate change; and to forestall maladaptation. Currently, many aspects of property development are indicative of maladaptation rather than adaptation. For example, property development can increase GHG emissions, and lead to path dependency (for example, large fixed infrastructure developments limit future changes) (Barnett \& O'Neill, 2010, p.212).

Urban development relates to climate change in at least three important ways. First, the product of development-the built environment-has climate impacts through various avenues including the materials used; and the design and location of developments. Second, the nature of the developer's products and the resulting built environment-such as its location, governance arrangements (e.g. covenants and bodies corporate)-influence the degree to which users are exposed to climate change hazards. Third, climate change presents direct and indirect challenges and hazards to which developers will need to adapt.

Most climate change research investigating urban socio-economic contexts has focused on household and community vulnerability, and on adaptive governance and policy tasks. Although a vast literature exists on climate adaptation policy, this has largely focused on the role of government actors and not on the private development sector and the financial sector that supports it. Thus, a major policy and knowledge gap exists in the social scientific understanding of private sector developers and financiers' capacity and capability to respond in an effective and timely way to the climate adaptation challenge.

Therefore, improving the knowledge of the capacity and weaknesses of private urban development and financing institutions in the area of climate change is a critical imperative within a wider policy of responsive climate change adaptation. Ignoring the private development sector and its institutional complexities would be to ignore a major potential source of improved adaptive activity.

\title{
1.1.1 Aim
}

The aim of this study is to investigate the institutional capacity of the private urban development sector, using South East Queensland (SEQ) as a case study to respond to the task of climate change adaptation, including the role that financial institutions play in this context. We anticipate the end users of this project will include developers, consultants, peak body representatives, Local and State Government, NGOs, and other interested members of the public

\subsubsection{Structure of this report}

This report is divided into 8 chapters. Following this introduction, Chapter Two (Research Activities and Methods) details the methods used in the research project. These included a questionnaire survey, a series of semi-structured interviews, and three focus groups. The chapter details the types of respondents who participated in the research and the firms to which they belong, and describes the methods used to analyse the data.

Chapters Three to Six discuss the themes and subthemes emerging from the research. These themes are grouped under four key headings:

- Climate change risks and the private urban development sector; 
- Institutional structure and the development process;

- Governance and regulation; and

- Finance and market.

Each theme and sub-theme is introduced by a brief literature review; followed by an overview of relevant survey results and a discussion that incorporates the results from the interviews and focus groups. This is then followed by a brief conclusion. For Chapter Four, Institutional Structure, some survey results (such as type of firm, and area of operation) are combined with the qualitative findings. When this is the case, the origin of the data is specified in the text of the report.

Chapter Three (Climate Change Risks and the Private Urban Development Sector), begins by briefly describing the issue of anthropogenic climate change, and the latest trends observed by key scientific institutions. It then describes the main potential risks that may arise from climate change, using four distinct categories; physical risks, economic risks, institutional and social risks. Each type of risk is explained by using existing literature and is further explored in the context of this study using the findings from the surveys, interviews and focus groups.

Chapter Four (Institutional Structure and the Development Process) details the institutional structure of the private property development sector, highlighting its heterogeneity. It begins by reviewing the characteristics of the participants in this study, in terms of the size and type of the firm they are associated with, and discusses how these factors influence their approach to risk management in general and in the context of climate change adaptation. The chapter also investigates how the specific characteristics of a firm play a role during the different phases of the property development process including; site selection, project planning, design and construction, financing, and marketing and sales. The chapter then briefly discusses the role that consultants play within the property development sector.

Chapter Five (Governance and Regulation) begins by reviewing the most relevant climate change related regulations and policies at national, state and local levels and discusses how developers and consultants are responding to them. Property developers operate within a diverse environment of complex legislation, regulation and economic forces. Climate change adds to this complexity, with changes to regulatory mechanisms aimed at supporting climate change adaptation and mitigation efforts. The chapter draws particular attention to the way in which the regulatory environment influences the adaptive capacity of individual firms. The chapter then briefly discusses how industry self-regulation and voluntary schemes influence a firm's adaptability.

Chapter Six (Finance and Market) focuses on the role that different sources of finance play in climate change adaptation. The role of financing is integral to the property development process, as the majority of developers are reliant on external funding. However, the interrelation of funding and development is a complex process, and is continually changing along with economic conditions. The chapter begins by briefly reviewing the major changes that occurred to property financing during and after the GFC and how these changes shape the nature of current development. It then describes the different sources of property finance in general and in the context of SEQ, and finally, how these influence climate change adaptation.

The final discussion Chapter Seven (The Adaptive Capacity of the Private Urban Development Sector) weaves together and synthesizes the disparate themes, and discusses how the findings of the research have implications for the adaptive capacity of the private property development sector in SEQ.

Chapter Eight (Conclusions, Recommendations and Future Research Directions) discusses limitations of the research, research gaps, and future research directions, and makes policy recommendations. 


\section{RESEARCH ACTIVITIES AND METHODS}

\subsection{Introduction}

This study used a mix of methods. Initially, a comprehensive review was conducted of the research literature, as well as on the "grey" literature (government reports, consultant publications, etc.). This result of this literature review was the Background Report (Taygfeld, Coiacetto, Shearer, Dodson, \& Banhalmi-Zakar, 2012).

Preliminary work also included the creation of a Project Reference Committee (PRC) to inform the process. The role of the PRC was to support the project; and comprised the research team, the EnviroDevelopment Manager of the Urban Development Institute of Australia, Queensland Chapter (UDIA), and representatives from the Planning Institute of Australia, Australia Green Infrastructure Council, Queensland State Government and the development industry (a large development firm). Other sector representatives were invited, but chose not to attend.

The PRC was an advisory, not steering body, and had the following roles.

- Advising on project direction.

- Assisting with project activities; in particular, identifying potential respondents for interviews and attending focus groups.

- Commenting on draft reports and the questionnaire survey.

- Supporting the fieldwork program including, where appropriate, acting as broker for contact with respondents.

Two meetings were held with the PRC, and some members also participated in the focus groups. The primary methods used in the research were a questionnaire survey, individual semi structured interviews, and focus groups. These methods are summarised in Figure 2 (below) and described in detail in the following sections.

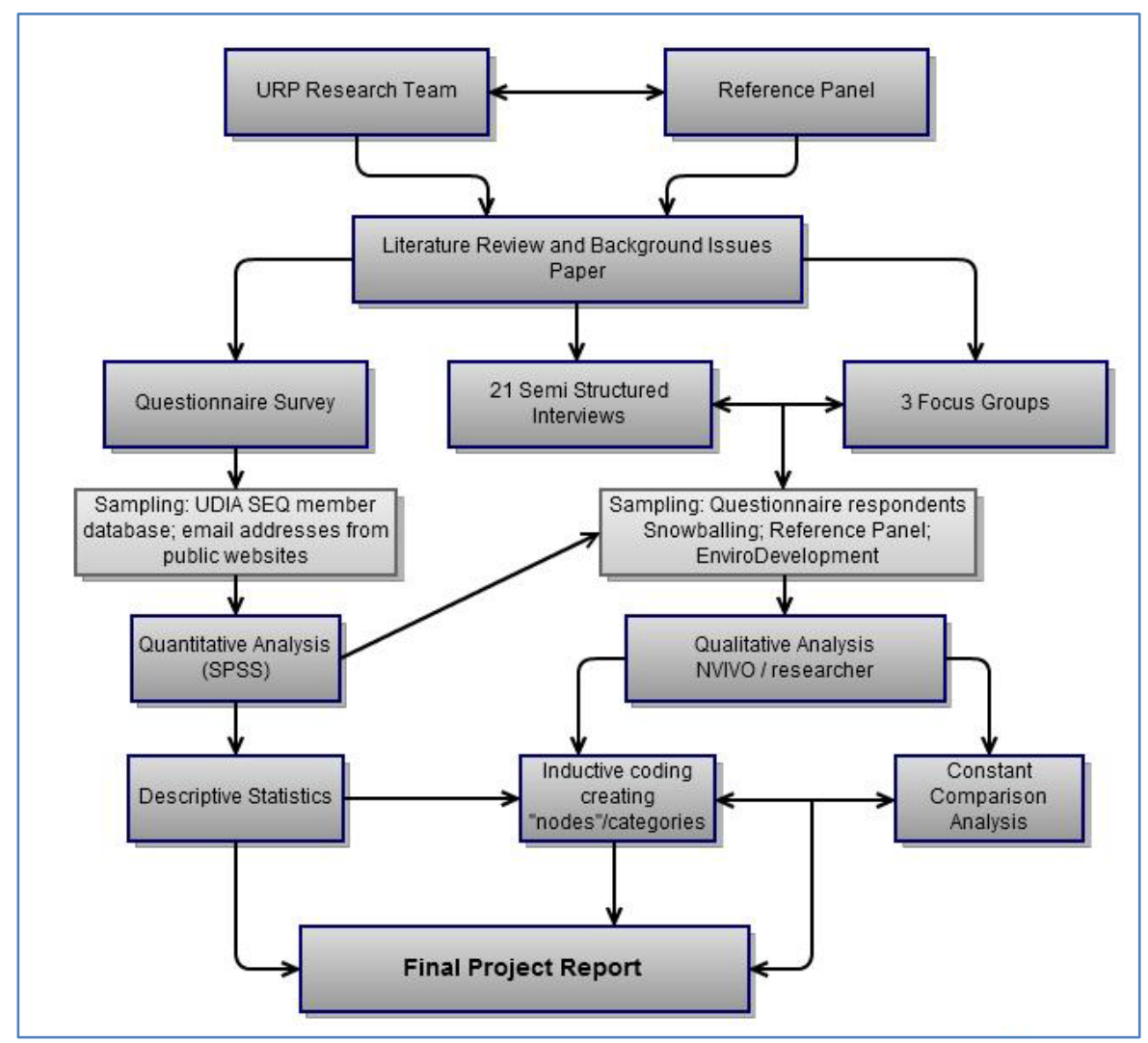

Figure 1 Schematic of Research Methodology 


\subsection{Methods: Questionnaire Survey}

A questionnaire survey was used to elicit information about the characteristics of development firms, and developer attitudes to climate change, adaptation to climate change and climate change risk. It included a final, open-ended question, which sought further opinions on climate change adaptation.

\subsubsection{Recruitment of Participants}

As the questionnaire survey involved human subjects, Griffith University Ethics approval for human research was applied for and granted (Protocol Number ENV/54/11/HREC). Informed consent was obtained through the standard online process, with a page giving information on the Ethical conduct of the survey, and consent indicated by "clicking" on the "Yes" button. The information page provided during the informed consent process included names of all researchers, and a contact name and details. All participants were assured of confidentiality and anonymity. The data was de-identified and no data provided by any participant would be published in a manner that could enable any third-party identification (protected anonymity). If requested, participants could obtain a summary of the research results.

The population from which possible participants for the questionnaire survey could be selected was members of the Queensland branch of the Urban Development Institute of Australia, (UDIA), and other SEQ developers, consultants and allied professionals who had contact details listed on public websites. The questionnaire could be completed by any employee of a firm.

The questionnaire survey was anonymous, and participants were not asked to provide any personal details. It is theoretically possible to identify a specific firm by using IP addresses, but the non-sensitive nature of the questions were deemed not to require any further security measures. Any IP data collected by the survey software was deleted.

\subsubsection{Development of Questionnaire}

The questionnaire was created using SurveyMonkey (SurveyMonkey, 2012) an online survey creation tool.

The questionnaire was constructed with reference to research questions within the approved project proposal (Dodson, Coiacetto, \& Banhalmi-Zakar, 2011); and the background paper (Taygfeld et al., 2012). Although similar research is relatively scant, the survey questions also referred to the recent paper by Taylor et al (2005).

\section{Piloting and Refinement}

The first drafts of the survey were pilot tested by chain referral sampling (using existing subjects to recruit further subjects) on a small population-members of the PRC and the research team-with a request to comment on length, comprehension, clarity and confusion. This was to identify ambiguous, repetitive, lengthy, or annoying questions (Francis et al., 2004).

After piloting, some questions were rewritten or modified; the meaning of some questions was clarified; and some were dropped to maintain a manageable length. Reasons for dropping questions included confusion in the pilot study and comments regarding relevance to the study.

\subsubsection{Format of the Questionnaire}

The questionnaire comprised six sections, with all questions optional. The full version of the questionnaire is provided in Appendix 9.5, but the questions are summarised as follows: 


\section{Pages One and Two}

The first two pages were information pages; the first with general information about the questionnaire, and the second with information about terms and conditions of the prize draw and the ethics information of the survey. Proceeding to page two was optional, but the question stated that clicking "No" implied consent to the ethical procedures on participation in the research. Details and hyperlinks to the Griffith University Ethics and Privacy web pages were also provided.

Page Three - About Your Company: Questions included: whether the respondent was a member of the UDIA, category of respondent (i.e. developer, consultant, etc.), number of staff, and annual numbers of development products completed.

Page Four - Development Profile: Questions included: areas in which the firm operated; type of property development; specialty (if any); and sources of funding. A multiple "Likert Scale" question asked what (general) issues were of significance to "you as a developer" and included finance and market changes, government regulation and climate change.

Page Five - Your Firm and Climate Change: Questions included: climate change knowledge; the degree of perceived threat to the firm; and knowledge of legislation and policy (differentiated into Queensland and Victoria) ${ }^{1}$. Two final Likert Scale questions asked to what extent the physical, direct hazards of climate change (i.e. floods and bushfire) and indirect hazards (i.e. changing regulation) might impact the firm.

Page Six - Adaptation to Climate change: Questions included: is adapting to climate change incorporated into decision-making and risk assessment; and if so, how? Further Likert Scale questions asked how firms saw their role in adapting to climate change; and what might encourage or discourage implementing adaptation measures.

Page Six contained a Question (34) designed to elicit qualitative responses, "Do you have any further comments?" It also included a final question (35) asking if respondents wanted to participate in further research and/or enter a prize draw. If answering "Yes" to any of these, the respondents were directed to a final page, for entering information (name, address, email address, etc.).

\subsubsection{Distribution of Questionnaire}

The questionnaire survey was distributed via the UDIA email newsletter list, the exact number of recipients of which is unclear, as these emails are likely to be circulated more widely than the initial "mailout"; for example, forwarded externally. It was also sent by direct email to 214 developers and allied professionals, such as consultants. Given that the UDIA newsletter posting only resulted in one response, for purposes of analysis, the number of surveys distributed is considered to be 215. A further tranche of questionnaires was sent to non-respondents some 4 weeks after the initial posting; in an attempt to obtain a larger returned sample percentage.

Of the total surveys emailed (and including one response from the UDIA mailout), 62 surveys out of 215 were returned. This resulted in an estimated final response rate of $28.84 \%$. This is relatively good, given that response rates for surveys have generally been decreasing over time, partly because of saturation of the potential surveyed audience (Krosnick \& Presser, 2010). Further, Taylor, et al. (2012) had recently surveyed the same population, so a degree of survey fatigue may have been evident.

\footnotetext{
${ }^{1}$ The original project proposal included Victoria; however, this was not included in the final draft due to difficulty contacting industry representatives.
} 
All questions were optional, and some applied only to specific respondents. Therefore, no one person completed every single question. Allowances were made for this in the statistical analysis, but for certain types of analysis, some respondents (16) were eliminated due to a large proportion of unanswered questions.

The questionnaire survey also offered an incentive in the form of a $\$ 150$ book voucher. The prize winner was selected randomly.

\subsubsection{Questionnaire Analysis Methods}

The questionnaire remained open for approximately $3 \frac{1}{2}$ months (April $13^{\text {th }}$ to 1 August 2012). As the initial distribution method of posting the survey link in the UDIA newsletter was unsuccessful, the individual emails were only sent to potential respondents from mid June 2012.

After the questionnaire was closed, the results were downloaded from the Survey Monkey website. Survey Monkey allows results to be downloaded in a variety of formats, included Excel, CSV, PDF, etc. It also allows results to be downloaded in expanded or condensed format. Condensed format places all answers in one column, except where the question was multiple choice, in which case it was expanded, with each answer choice assigned to a column. Questions can also be exported in numerical or text format, with numerical values assigned to questions in the order of choice. For this exercise, the results were downloaded in condensed, text format, and conversion to numerical format was done manually.

The downloaded Excel file was imported into SPSS (v20) statistical software. The first step was to ensure the questions were numbered the same as in the questionnaire. Second, all questions were coded numerically. When choices for questions were given on a Likert scale; the answers were manually coded and weighted according to the question. For example, for each of the 15 choices given in Q19: What issues are of most significance to you as a developer; the "Likert Scale" options given were: very important, important, neutral, not important, very unimportant. To code the questions, very important was rated 5 , to very unimportant, which was rated 1 . Missing values were not coded because SPSS automatically codes and accounts for missing values during statistical analysis procedures. Questions were also categorised according to the type of variable, for example, Nominal, Ordinal or Scale (Ratio and Interval data are treated the same in SPSS, and termed Scale).

Third, each question was given a unique name (i.e. Q1A, Q1B) and all possible values listed in the values field. For example, Q3: Category of Respondent; was coded as:

1. Developer/Builder $<\$ 10$ million turnover

2. Developer/Builder $>\$ 10$ million turnover

3. Developer/Builder $>\$ 20$ million turnover

4. Consultant

5. Institution or Non Profit

6. State School / Local Government / Utility Authority

7. Statutory Authority.

\section{Statistical Analysis}

After coding and cleaning the data, basic descriptive analysis was conducted using SPSS to calculate statistics such as mean, frequencies and simple correlations. Answers to questions such as Q15: Type of development; Q16: Development product and Q17: Do you specialise in any residential market sectors, were graphed to better visualise the responses. The majority of questions were subjected to descriptive analysis only, as this research was primarily exploratory, and the dataset was too small for more complex statistical analysis. 
For much of the statistical analysis, the results were split according to the category of respondent. These categories were based on answers to Question 3 (Category of Respondent) and included; Large Developer (turnover $>\$ 20$ million, $n=4$ ); Medium Developer (turnover $>\$ 10$ million and $<\$ 20$ million, $n=8$ ); Small Developer (turnover $<\$ 10$ million, $n=7)$; and Consultant $(n=27)$. The aim of this was to differentiate responses from developers and consultants.

For the following questions, the answers were combined in a single table (Table 2, Ranked and Categorised Risks, Survey Respondents).

- Question 19: What issues are of most significance to you as a developer?

- Question 26: To what extent would the following primary hazards of climate change impact the development activity of your firm?

- Question 27: To what extent would the following secondary hazards of climate change impact the development activities of your firm?

For this combined analysis, as well as for Question 30 (How does your firm see its role in adapting to climate change?); Question 31 (Which of the following would make your firm more likely to implement climate change adaptation measures?) and Question 33 (How likely are the following to prevent your firm implementing climate change adaptation?); one way Analysis of Variance Analyses (ANOVAs) were conducted.

\subsection{Methods: Semi Structured Interviews}

\subsubsection{Recruitment of Participants}

A number (21) of interviews were conducted in order to further explore and obtain an in-depth understanding of the adaptive capacity of the SEQ property development industry. These interviews also sought to further explore the wide range of interconnected drivers and barriers to the adaptive capacity of the sector.

The initial participants were contacted by the EnviroDevelopment Manager of the UDIA (Queensland). They were then emailed by the research team, and meetings organised. Other participants ticked "yes" to the Further Research section in the questionnaire. Other participants were recruited by snowballing, either at seminars and workshops, through personal networks, or by asking interviewees to recommend people.

Each interview lasted approximately one hour, and was generally held at the interviewee's place of work, or a nearby coffee shop. When permitted by the interviewee, the interviews were recorded using a "Voice Recorder" application (app) on the investigator's mobile telephone. Interviewees were given an information sheet, and asked to sign a disclaimer (Appendix 9.1).

\subsubsection{Format of the Interview}

A series of questions based on the project proposal and the draft report were prepared prior to the interviews, and partially crafted according to the known characteristics of the respondents (Appendix 9.2). The first half of the interview questions related to the development activities and company structure of the interviewee, and the second half to climate change adaptation. When it was known that the interviewee had completed the survey, the questions about the company structure were omitted or shortened. However, because the survey was anonymous, the researchers had no way of knowing this information, unless the respondent chose to inform them.

Generally, two members of the team conducted the interviews, but at times, due to conflicting schedules, only one team member attended. The interviews began by the researcher/s introducing themselves. The researchers explained the research, and what they were hoping to find out. They also reiterated that the interviews were confidential, and that no identifying material would be published. 
They discussed the ethics and privacy provisions of the research, and asked permission to conduct the interview, and record the interview. When participants requested no recording, the interviews were manually transcribed.

Questions about the development activities and structure of the firm included questions on the internal structure of the firm; types of development and other activities of the firm; the geographical scope of operations; challenges and risks for development; and sources and issues regarding financing. The interview process then moved on to climate change and climate change adaptation. The introduction to the section was paraphrased from the following:

"One issue that we think might have a big impact on development is the big question of climate change. How it could affect development is not clear-and we are interested in exploring that with you. We are also interested to know how you think developers could get ready for it and what it means for the industry. We believe that climate change could have direct impacts on development (e.g. from severe weather events and sea level rise) but also indirect affects thought new rules and regulations, through markets (changing consumer demand, through lending and borrowing, etc.)" (Research Team, 2012)

Questions included the participant's thoughts on what climate change might mean to their business; their attitudes and perceptions towards various direct and indirect climate change risks, and how these might be managed; questions on finance availability; whether insurance and litigation had had any impact on the firm; market demand for greener products; attitude to the regulatory environment; and what climate change adaptation meant to them.

These questions are reproduced in detail in Appendix 9.2. Of note, the interviews were semi-structured, so not all questions listed were used for each respondent, and other questions not listed, were asked.

\subsubsection{Interview Analysis Methods}

All interviews were recorded, and manually transcribed, using Express Scribe transcription software and a foot-pedal controller. The transcriptions were then uploaded into Nvivo (v10) software and key words, paragraphs and phrases were categorised under "Nodes", which related to common themes in the interviews and focus groups, and to the chapter structure of this report.

Nvivo is a qualitative data analysis software from QSR International; and is designed to analyse rich, large datasets of text, audio and video recordings, surveys and other media (QSR International, 2012). Nvivo has a number of search, query and visualisation tools, and is extensively used in qualitative research (Hughes, Lewins, \& Silver, 2010; QSR International, 2012). This is explained fully in the following Section 2.4 Methods: Focus Groups, as the analysis used was the same for both methods.

\subsection{Methods: Focus Groups}

The aim of the focus groups was to gather opinions, beliefs, and attitudes about issues pertinent to the research (e.g. the capacity of the private property development sector to adapt to climate change) and to encourage discussion about climate change adaptation and the private property development sector in general.

Three focus groups were conducted. It was originally aimed to differentiate the participants by category; i.e. developers, consultants and peak bodies (i.e. UDIA, Australian Green Infrastructure Council, Planning Institute of Australia, State Government). However, due to difficulties with scheduling, the eventual focus groups 
were more diverse, with representatives from more than one category. The average number of participants was 3.

\subsubsection{Recruitment of Participants}

Participants for the focus groups were recruited by snowballing previous interviewees (see above); as well as targeting respondents who expressed an interest in further participation by indicating so in the survey.

\subsubsection{Format of the Focus Groups}

Participants in the focus group signed a disclaimer and consent form (Appendix 9.1). Each focus group lasted approximately 90 minutes. A series of questions was asked, and discussion was encouraged. Participants were also reminded that, like the interviews, the focus groups were confidential, including confidentiality between attendees.

The first focus group comprised three owners, or staff in the development section of developer firms, and one representative from a quasi-government development firm. The second focus group was intended to target consultants and peak bodies. However, limited numbers of participants were able to attend. Therefore, in order to get a wider diversity of responses, the third focus group comprised a range of participants, including a developer and two consultants.

A member of the research team acted as facilitator; and the other team members asked additional questions, and transcribed the proceedings. To begin, the researchers briefly described the project, and introduced themselves. Participants were then encouraged to give their names, where they worked, and describe the type of development work they conducted (or type of consulting). Then, a series of openended questions were asked, with subsequent questions based on participant responses.

The starter questions differed according to the makeup of the focus group; for example, questions asked of consultants differed from those asked of developers. Where the focus group comprised representatives from more than one category, questions were adapted accordingly. A number of different questions were asked, and included:

- What is the timescale and spatial scale of the projects on which you work?

- How does financing set the parameters for what the developer can and cannot do?

- Can you comment on changes to policy and regulation, the capacity of developers to respond to changes in regulation or political uncertainty?

- How do you believe climate change is likely to affect the property development sector?

Some focus group questions are summarised in Appendix 9.3. Of note, the focus groups were more informal than the interviews, so the range of questions was large, and dependent on the mix of participants. Therefore, the exact text of the questions is not included.

\subsubsection{Focus Group and Interview Analysis Methods}

The interviews and focus groups were also recorded. This recorded material (or notes from meetings when participants requested no recordings) was then manually transcribed into Microsoft Word 2010, using Express Scribe software. The questions asked by the interviewers were not transcribed verbatim, but in dot points, where necessary. When possible, an interviewee's responses were transcribed verbatim; however some recordings were made outdoors and the sound quality was affected by external noise. 
The transcripts were then imported into Nvivo (v10) software. The primary method used was a form of qualitative content analysis, the "constant comparison" approach. However, as described in Section 2.2, the research also included quantitative methods, specifically in analysing the questionnaire survey.

This analysis primarily used inductive coding in Nvivo rather than deductive coding. Deductive coding in Nvivo is customarily used in hypothesis-driven research, when the answer to a specific research question is required. Inductive (or bottom up) coding is used more often in exploratory research such as this study, when many aspects of an issue (i.e. a developer's capacity to adapt to climate change) are investigated (Walsh, 2002; Wiltshier, 2011; Zhang \& Wildemuth, 2009).

A number of methods exist for text coding including; classical content analysis, word count, keywords-in-context, domain analysis, taxonomic analysis, componential analysis and the constant comparison approach (Wiltshier, 2011; Zhang \& Wildemuth, 2009). For example, keywords-in-context is more commonly used when the data is in the form of short, one-phrase answers, or when researchers wish to investigate the frequency of use and understanding of specific words. Domain analysis is used when researchers want to explore the symbology and cultural significance of different words or phrases, and is more commonly used in ethnographic studies. Taxonomic analysis is usually conducted subsequent to domain analysis. Classical content analysis is similar to constant comparison analysis, except the nodes and themes are also counted (Owens, 2012; Zhang \& Wildemuth, 2009).

The majority of these pertain to specific research methodologies, which were not relevant to this study. Therefore, as indicated above, the coding was informed by the "constant comparison" approach. Constant comparison is one of the most common qualitative data analysis techniques used for text coding (Zhang \& Wildemuth, 2009). It was developed by Glaser and Strauss (1967) and further modified over time (Owens, 2012). In this method, inductive reasoning is used to derive categories (or nodes/themes) from the data. The text is broken down into discrete categories, which can be used in both descriptive and explanatory analysis.

In brief, the process used for the constant comparison method was as follows. First, five free "nodes" (or themes) were created. A node in Nvivo is essentially a category, and can either be singular (a free node), or sub (tree) node, situated within a hierarchical structure. Common themes were based on the Background Project Report (Taygfeld et al., 2012) and further refined at a number of meetings of the research team, to test their validity and robustness. These categories were used to set up the high level free node structure, and also informed the headings in the results (Chapters Three to Seven) of this document. The top level nodes were Climate Change, Institutional Structure, Governance, Finance and Market, and Adaptive Capacity.

Having set up the top level free nodes, each coder worked individually, creating their own Nvivo project. Each primary coder read the texts and categorised comments under each free node, in an inductively derived node structure. Each coder's structure was then merged, to form the final project file. As the coders worked through more of the data, new nodes were created, and existing, similar nodes were merged. Nodes were also moved between higher-level categories. The creation of this categorical node hierarchy was a fluid process, and was only finalised at the end of coding, and after a project team workshop. The final node hierarchy is illustrated in Table 1. 
Table 1 Final Themes (Nodes) and Sub Themes Identified in Qualitative Analysis

\begin{tabular}{|c|c|}
\hline Top Level Node & Sub Nodes \\
\hline \multirow[t]{7}{*}{ Climate Change } & Attitude and perceptions of climate change \\
\hline & Bankers Responses \\
\hline & Direct Risk \\
\hline & Indirect Risk \\
\hline & Regulatory risks \\
\hline & Sea Level Rise \\
\hline & Site risks \\
\hline \multirow{6}{*}{$\begin{array}{l}\text { Institutional Structure and } \\
\text { Governance }\end{array}$} & Consultants and Other Stakeholders \\
\hline & Developers General \\
\hline & Developer structure \\
\hline & Development process \\
\hline & Development Type \\
\hline & Land banking \\
\hline \multirow[t]{7}{*}{ Governance and Regulation } & Building Codes \\
\hline & Coastal Plan \\
\hline & Local Government \\
\hline & Regulation, Legislation and Planning \\
\hline & Time Delays \\
\hline & Industry Self-Regulation \\
\hline & Political environment and Government \\
\hline \multirow{5}{*}{$\begin{array}{l}\text { Economics, Finance and } \\
\text { Market }\end{array}$} & GFC and economic climate \\
\hline & Green and Market Demand/ Sustainability \\
\hline & Marketing \\
\hline & Sources of Finance \\
\hline & Valuation \\
\hline \multirow[t]{12}{*}{ Adaptive Capacity } & Adaptive Measures and Responses \\
\hline & Community and Social \\
\hline & Barriers to adaptation \\
\hline & Cost of adaptation and mitigation \\
\hline & Attitude \\
\hline & Communication, Information and Knowledge \\
\hline & Drivers, motivation \& opportunities for adaptation \\
\hline & Environmental Concern \& Sustainability \\
\hline & Insurance and Legal \\
\hline & Leadership, Legacy and Reputation Branding \\
\hline & Returns for adaptation \\
\hline & Systems approach \\
\hline
\end{tabular}




\section{CLIMATE CHANGE AND THE PRIVATE URBAN DEVELOPMENT SECTOR - RISKS AND OPPORTUNITIES}

\subsection{Introduction}

It is now widely accepted that the climate is changing, largely due to the release of greenhouse gases from human related activities (Braganza \& Church, 2011; IPCC, 2011). The term greenhouse gas (GHG) is most commonly used to refer to carbon dioxide $\left(\mathrm{CO}^{2}\right)$, but also includes methane, water vapour, nitrous oxide and ozone (Kiehl \& Trenberth, 1997).

Records of global temperatures reveal that, over the past 60 years, average temperatures in Australia have increased by about $0.7^{\circ} \mathrm{C}$ (Della-Marta, Collins, \& Braganza, 2003); and during the past 100 years, global sea levels have risen by an average of $1.7 \mathrm{~mm}$ per year (Bindoff et al., 2007). Changes have also been recorded in rainfall patterns, the frequency of extreme weather events,; and rises in oceanic temperatures, salinity and acidification (Bindoff et al., 2007; Durack \& Wijjfels, 2010).

Scientists have repeatedly warned that a failure to immediately reduce $\mathrm{CO}^{2}$ emissions would trigger abrupt and irrevocable changes in climatic conditions-a tipping pointwhereby temperatures will increase beyond $2^{\circ} \mathrm{C}$, exposing humanity to disastrous consequences (Hansen, 2008; Hastings \& Wysham, 2010; IPCC, 2007). A recent report, released by the World Bank, sends a stark warning that the world is facing $4^{\circ} \mathrm{C}$ of climate warming by the end of the century, which will be manifested in 'unprecedented heatwaves, severe droughts, and major floods in many regions, with serious impacts on ecosystems and associated services' (World Bank, 2012b, p. 16).

Ignoring climate change is therefore no longer a responsible response, given the severe social, economic and environmental implications of inaction (CSIRO, 2011; Garnaut, 2011; IPCC, 2007). Across the globe, efforts to mitigate (i.e. reduce GHG emissions) are currently underway. However, as indicated by the World Bank report, and other recent data, these mitigation measures are largely failing to curb GHG emission levels, which continue to track higher. Thus, some degree of climate change is unavoidable and adaptation should be considered an essential complementary measure to mitigation (Barnett \& O'Neill, 2010; Smit \& Pilifosova, 2003).

\subsection{Climate Change Risks and Opportunities}

Climate change could result in serious consequences for the urban environment and for the private urban development sector. For urban property developers, climate change presents a range of challenges - and even potential opportunities-which arise directly from the physical changes in the climate and indirectly from the intensifying regulatory and social responses to climate change.

The built environment is influenced by climate in many ways through its location, form and design considerations. For example, developments located along the coastal foreshore are exposed to storm surges and sea level rise; whereas developments located in heavily vegetated areas could be exposed to bushfire risks and severe weather events. Building materials and other design considerations, such as height and aspect, influence the level of exposure to extreme weather events and the potential damage associated with such events.

The 2011 and 2013 Queensland floods and the 2009 Victorian bushfires, while not definitively having a climate change 'fingerprint', provide an important reminder on how severe weather events could impact urban infrastructure and property. Similarly, governmental and societal responses to climate change could also present developers with a new set of challenges, ranging from new regulations and policies to changes in consumer preferences. These potential risks may be considered as indirect risks 
because they do not rise directly from the physical changes to the climate, but occur as society responds or adapts to climate change.

For example, in February 2012, the Queensland government introduced the (now superseded) Queensland Coastal Plan (the Coastal Plan), which was a statutory document stipulating a range of restrictions with respect to future developments along the Queensland coast. The plan was developed partially in an attempt to protect future developments from the impacts of projected climatic changes in these areas (The State of Queensland, 2012b). The release of the plan, however, created a strong sense of confusion, uncertainty and anger amongst developers and allied professions; they said it was developed and implemented too hastily, giving developers little or no time to adjust (Property Council of Australia (PCA), 2012).

The Coastal Plan may typify a new feature of regulatory changes in the context of climate change, as the pressing nature of some of the risks may not enable a gradual or incremental change in regulations. Developers, on their part, may have to be prepared to operate in an uncertain regulatory environment, whereby changes to legislation, planning schemes and building codes are commonplace.

However, as the property development sector is highly heterogeneous and complex, the risks and opportunities arising from climate change may differ markedly according to the location of development with respect to climate risks (i.e. on the coast), the nature of the development, financing and insurance arrangements, and the specific characteristics of the firm or individual who is undertaking the development.

In this context, the way that property developers view climate change as a risk to their operation and product is a key question that this chapter explores. We answer this question, first by using the literature to provide a generic overview of some of the potential risks that developers may face as a result of climate change; and second, through the surveys, interviews and focus groups with developers and allied professionals with respect to those potential risks.

As mentioned above, there are two broad risk categories: direct and indirect. While it is reasonably easy to define direct risks, indirect risks are somewhat harder to define and are therefore further divided into three subcategories: economic, institutional and social risks. Accordingly this chapter discusses these risks under four broad risk categories, namely:

1. physical risks (i.e. flooding, coastal storm surges, cyclones and fire);

2. economic risks (i.e. insurance, market demand, lending and litigation);

3. institutional risks (i.e. regulations, policies, industry structure); and

4. social risks (i.e. marketing, green demand, and reputation).

This chapter begins with an overview of the survey results with respect to how developers rank these different types of risks and continues with a discussion that incorporates the results from the interviews and focus groups.

\subsubsection{Combined Risks - Results from Survey}

The results from the questionnaire survey provided a useful representation of how developers and consultants rank direct and indirect climate change related risks. The questionnaire survey asked a range of questions on the different types of risk faced by developers, and those from other allied or intermediary roles, such as consultants. 
These questions were rated on 5 point Likert scales ranging from very unimportant (1) to very important (5).

The results in Table 2 represent a combination of 3 questions (all of which were on the same scale ${ }^{2}$ ):

- What issues are of most significance to you?

- To what extent would the following primary hazards of climate change impact the development activity of your firm?

- To what extent would the following secondary hazards of climate change impact the development activities of your firm?

The respondent were differentiated into four categories; small, medium and large developers, and consultant (all others). The most important category of risk identified by survey respondents (of all types) was institutional risk; and of these, the three most significant were changed (lengthier) approval times, more onerous government charges, and regulatory risk. Of note, the first two can also be seen as economic risks.

Most respondents ranked the above risks similarly. However, some differences in responses were noted between large developers and consultants (which were relatively consistent) and small and medium developers. This is not unexpected as many large developers have a range of staff roles, and surveys on climate change may well be answered by specialist staff such as sustainability managers. In many cases, such a respondent from a large development firm may perform a similar role to a specialist consultant (i.e. a sustainability manager).

The second ranked category was economic risks. Of these; changed market demand, higher government charges, and changes in valuation; were considered the most important risks to developers. Of note, these categories are not discrete, and there was some overlap; many risks can be considered both institutional and economic.

Larger developers were also concerned about risks such as changes to taxation regimes, while medium and smaller developers felt that value loss from location (i.e. changes to flood lines) and interest rate changes were important. Consultants were neutral on most economic risks, although they did rank economic risk in general, and higher government charges as important to their firm. With regard to changes in market demand for greener properties (which may also be categorised as an economic risk), participant responses were predominantly 3 (neutral), except for medium developers who ranked this as 4 (important).

Finally, direct (physical) risks were mostly ranked 3 (neutral) or 2 (unimportant), suggesting they were not seen as a critical issue. These are discussed in more detail in the following section; however larger developers and consultants tended to view physical risks as more important than small and medium developers. This is likely related to the greater spatial and temporal scale of their developments. In addition, the higher rankings of flooding, cyclones and extreme rainfall are likely an effect of the greater salience of the Brisbane floods and Cyclone Yasi in 2010/2011.

An Analysis of Variance (ANOVA) was conducted on the means of the different physical risks. There were no significant differences between groups, with the exception of sea level rise $(F(3,31)=2.927, p=.049)$, which was likely due to the very low rankings given to this by small developers.

\footnotetext{
${ }^{2}$ The total $(\mathrm{n}=35)$ is less than the total number of respondents who answered the survey as, respondents who did not complete all three questions were excluded for this exercise.
} 
Table 2 Ranked and Categorised Risks from Survey Respondents

\begin{tabular}{|c|c|c|c|c|c|c|}
\hline $\begin{array}{l}\text { Risk } \\
\text { Category }\end{array}$ & & $\begin{array}{l}\text { Large } \\
(n=4)\end{array}$ & $\begin{array}{l}\text { Medium } \\
(n=6)\end{array}$ & $\begin{array}{l}\text { Small } \\
(n=6)\end{array}$ & $\begin{array}{l}\text { Consultant } \\
(n=19)\end{array}$ & $\begin{array}{l}\text { Total } \\
(n=35)\end{array}$ \\
\hline & & \multicolumn{3}{|c|}{ Developers - Total $n=16$} & & \\
\hline \multirow{2}{*}{$\begin{array}{l}\text { Social } \\
\text { Mean }\end{array}$} & \multirow[t]{2}{*}{ Green Demand } & 2.8 & 4.0 & 3.0 & 3.6 & 3.5 \\
\hline & & 2.8 & 4.0 & 3.0 & 3.6 & 3.5 \\
\hline \multirow{13}{*}{ Institution } & Competition & 3.5 & 3.4 & 2.6 & 3.8 & 3.5 \\
\hline & Carbon Tax & 4.3 & 3.5 & 4.2 & 3.3 & 3.6 \\
\hline & Post Development Risks & 4.3 & 4.4 & 3.5 & 3.7 & 3.9 \\
\hline & $\begin{array}{l}\text { Property Value loss from } \\
\text { Legislation }\end{array}$ & 4.5 & 4.5 & 4.2 & 3.6 & 4.0 \\
\hline & Land Use Planning \& Zoning & 4.3 & 3.8 & 3.3 & 4.3 & 4.0 \\
\hline & More onerous Building Codes & 4.3 & 4.4 & 3.7 & 4.0 & 4.1 \\
\hline & Indirect Climate Change & 4.5 & 3.8 & 3.5 & 4.3 & 4.1 \\
\hline & Delays from Climate Change & 4.5 & 4.6 & 4.0 & 4.0 & 4.2 \\
\hline & More Regulation & 4.3 & 4.8 & 4.5 & 4.4 & 4.5 \\
\hline & Regulatory Risk General & 4.7 & 4.1 & 4.5 & 4.7 & 4.5 \\
\hline & Government Charges & 4.8 & 4.8 & 5.0 & 4.4 & 4.6 \\
\hline & Approval Times & 4.8 & 4.9 & 4.8 & 4.8 & 4.8 \\
\hline & & 4.4 & 4.2 & 4.0 & 4.1 & 4.1 \\
\hline \multirow{13}{*}{ Economic } & Insurance Risk & 2.3 & 2.8 & 2.8 & 3.2 & 2.9 \\
\hline & High Australian Dollar & 2.5 & 3.1 & 2.8 & 3.0 & 2.9 \\
\hline & $\begin{array}{l}\text { Property Value Loss from } \\
\text { Location }\end{array}$ & 3.0 & 4.0 & 2.8 & 3.3 & 3.3 \\
\hline & Insurance Difficulties & 3.5 & 4.0 & 3.0 & 3.5 & 3.5 \\
\hline & Tax Risk & 4.5 & 3.6 & 4.3 & 3.1 & 3.6 \\
\hline & Economic Risk (GFC) & 3.8 & 3.9 & 3.8 & 4.1 & 3.9 \\
\hline & Interest rate changes & 4.0 & 4.3 & 4.5 & 3.6 & 4.0 \\
\hline & Financial Risk & 3.5 & 4.4 & 4.5 & 3.9 & 4.1 \\
\hline & Finance Difficulties & 3.8 & 4.3 & 4.8 & 3.8 & 4.1 \\
\hline & Valuation & 4.3 & 4.3 & 4.3 & 3.9 & 4.1 \\
\hline & Higher Government Charges & 4.3 & 4.8 & 4.7 & 4.2 & 4.4 \\
\hline & Changed Market Demand & 4.5 & 4.8 & 4.7 & 4.5 & 4.6 \\
\hline & & 3.7 & 4.0 & 3.9 & 3.7 & 3.8 \\
\hline \multirow{10}{*}{ Direct } & Higher temperatures & 2.3 & 2.7 & 2.2 & 2.7 & 2.5 \\
\hline & Drought & 2.3 & 3.0 & 2.0 & 2.7 & 2.6 \\
\hline & Bushfire & 2.0 & 2.7 & 2.5 & 2.7 & 2.6 \\
\hline & Storms (hail and wind) & 2.5 & 2.8 & 2.3 & 3.0 & 2.8 \\
\hline & Sea level rise & 2.3 & 2.7 & 1.5 & 3.4 & 2.8 \\
\hline & Storm surge & 2.3 & 2.8 & 2.0 & 3.3 & 2.9 \\
\hline & Storms (cyclones) & 2.5 & 3.5 & 2.7 & 3.1 & 3.0 \\
\hline & Extreme rainfall & 3.5 & 3.2 & 2.5 & 3.4 & 3.2 \\
\hline & Direct Climate Change & 2.8 & 2.8 & 2.8 & 3.8 & 3.3 \\
\hline & Flooding & 3.0 & 3.0 & 2.2 & 3.8 & 3.3 \\
\hline Mean & & 2.5 & 2.9 & 2.3 & 3.2 & 2.9 \\
\hline
\end{tabular}




\subsubsection{Physical Risks}

At larger scales, developers are exposed to risks and climate change because of the spatial location of their developments; and at smaller scales, because of the characteristics of the development sites. For example, a developer specialising in marina developments in North Queensland may be exposed to sea level rise, storm surge and cyclonic activity; whereas one specialising in retirement units in central Queensland may be exposed to bushfires, drought, heat stress and inland flooding.

In the survey, few respondents thought that the physical hazards of climate change would pose a significant threat to their firm (Figure 3). Most rated these as 3 (neutral) to 4 (unimportant). Of these, the most significant threats were flooding, extreme rainfall, cyclones and storm surge. However, it was mostly the consultants who viewed these as a greater threat than the developers, and in particular, small developers.

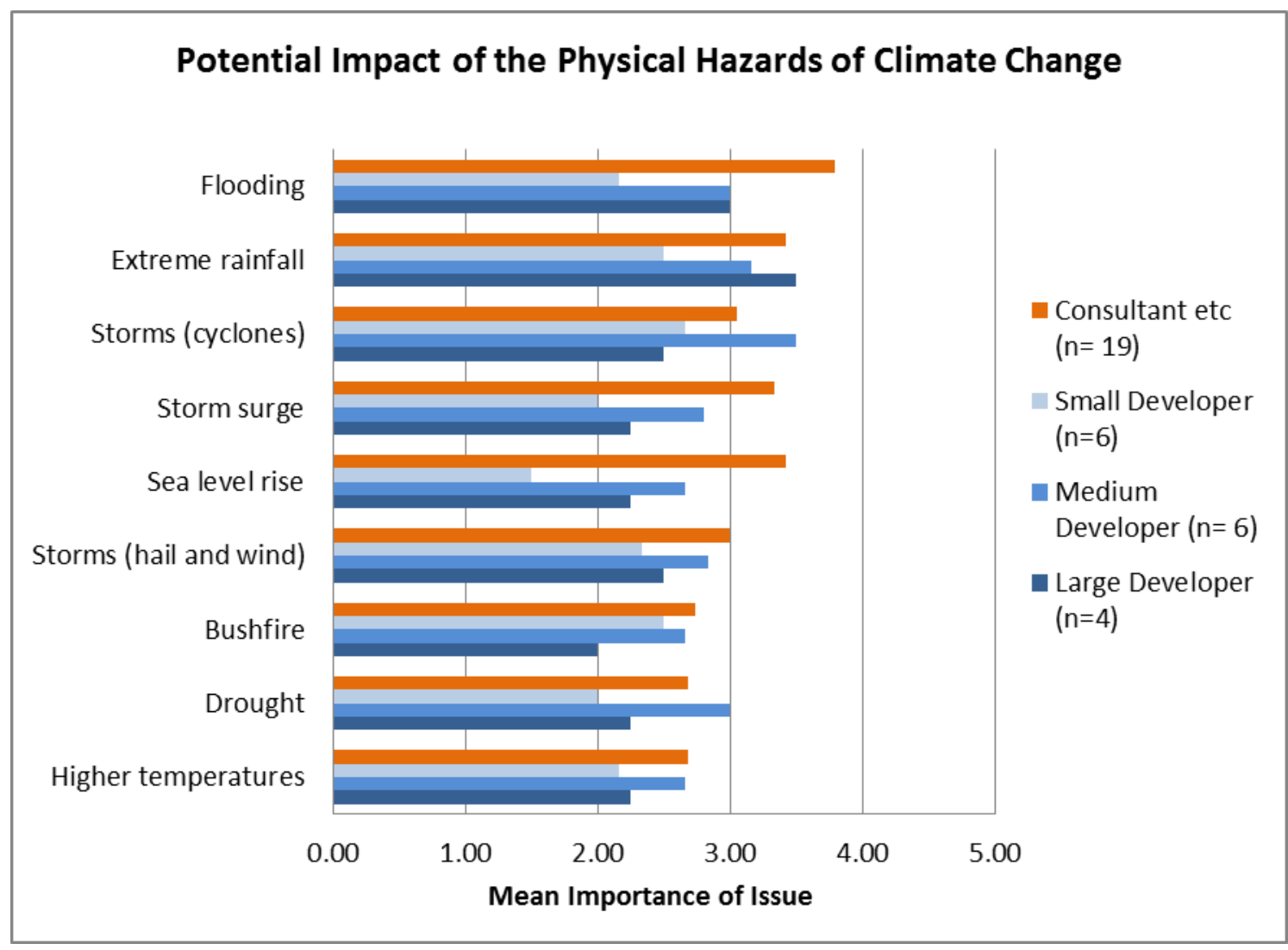

Figure 2 Potential Impact of the Physical Hazards of Climate Change (by respondent type)

\section{Flooding}

There is currently much debate in the Australian insurance industry about the appropriate definition for flood. This debate surfaced as insurance companies struggled to cope with repayments to cover the severe damage caused by the Brisbane floods in January 2011. The present report defines flooding according to the Geoscience Australia definition:

A general and temporary condition of partial or complete inundation of normally dry land areas from overflow of inland or tidal waters from the unusual and rapid accumulation or runoff of surface waters from any source ((GA), 2001). 
Every year in Australia, floods cause millions of dollars of damage to buildings and infrastructure. The Insurance Council of Australia estimates the cost to the insurance industry of the 2011 Queensland's floods at $A \$ 2.4$ billion (ICA 2011, p.1). This is only a small fraction of the total cost to the Australian economy, which was estimated at between $\$ 13$ and $\$ 30$ billion (PwC, 2011, p.2).

While it is difficult to project with certainty that flood events will increase in the future as a result of climate change, a recent report by the IPCC (2011) suggests that:

There is medium confidence (based on physical reasoning) that projected increases in heavy rainfall would contribute to increases in local flooding, in some catchments or regions...[and] There is high confidence that locations currently experiencing adverse impacts such as coastal erosion and inundation will continue to do so in the future due to increasing sea levels, all other contributing factors being equal. ((IPCC), 2011, p.12).

Development in or near flood prone areas (coasts, wetlands and rivers) may increase flood risk due to three factors: (1) development can destroy natural barriers such as mangroves and sand dunes to be replaced by artificial ones (e.g. dykes/levees or sea walls); (2) development increases the number of people and extent of infrastructure exposed to flood risk and damage; and (3) artificial barriers may prove to be inadequate under more extreme weather events, as was the case in the May 2011 tsunami event in Japan, Hurricane Katrina in 2005 and the 1995 river flooding in the Netherlands. In all of those events, existing flood mitigating barriers failed, with catastrophic results (Nicholls et al., 2007).

State and local governments across Australia have put into place a number of policies that attempt to protect buildings and infrastructure in flood-prone areas. In Queensland, the Coastal Plan attempted to restrict development along coastal areas that are vulnerable to sea level rise, storm surges and flooding ${ }^{3}$. Local planning schemes also stipulate restrictions with regards to the appropriate height and other design factors for buildings in flood prone areas.

Regulatory provisions that aim to address the risk of flooding are generally well accepted by developers. However, this study found that while developers are happy to comply with codes and regulations that are clear and sensible (i.e. those which can be resolved with relatively simple engineering solutions), they were highly sceptical and critical about the Queensland government initiative to prohibit development in "Coastal Hazard Areas" as stipulated in the Coastal Plan (The State of Queensland Department of Environment and Resource Management (DERM), 2012a, p.44).

With respect to flooding, many participants mentioned that they had lived with flooding before and that an 'extra flood here and there' is not likely to present a real issue to developers. Exposure to floods, they argued, is influenced not only by the location of the development but also by the duration of the development process. As most developers aim to be 'in and out' as quickly as possible, that is, one or two years, their actual exposure to flooding risk is minimal and does not warrant special consideration. In addition, if flooding does occur during the development process, then the developer or builder assumed it would be covered by their insurance.

\footnotetext{
${ }^{3}$ The Queensland Coastal Plan has been suspended from operation since October 2012.
} 
This may be slightly different for large scale developments that span 25 years or more. Companies involved with this type of developments (e.g., master planned developments) are likely to consider flooding more seriously because of the higher likelihood of experiencing it during the development process, but also because their reputation may be at stake if a significant flooding event impacts their development, and the community living within.

Although it is unlikely that developers will go beyond what is required from them by regulations and building codes to address issues such as flooding; there appears to be a 'natural process of adaptation', whereby an event such as the Brisbane floods influences the design of new developments to comply with new regulations and in some cases, insurance demands. One participant discussed at length how certain extreme weather events in the past, including flooding, shape the way we design and build houses in affected areas, drawing some examples from the tornado that swept through the Brisbane suburb of Tarragindi in the early 80s, as well as the 2011 Brisbane floods.

"The flooding last year is driving a new form of house construction in areas that are prone to extreme flood events, so your downstairs areas become sacrificial, becomes a vast open space that the water just goes through, all your surfaces can be wet so you don't do Gyprock in those areas any more, you do rendered walls or something like that, so the floods come through and disappears and then you hose it out and start using it again" (CS6, Consultant).

In this study, developers were generally extremely risk averse, particularly when economic conditions are not in their favour (e.g., the GFC period). Attempts to restrict or prohibit development in flood prone areas simply means that some developers will seek to develop land in areas where regulatory restrictions are not as costly or risky. One participant mentioned a developer who 'ran to the hills' when the Coastal Plan came into effect. Most small developers do not have land banks and there is little chance that they would expose themselves to additional risk by purchasing land in areas that are likely to be impacted by restrictive regulations.

On the other hand, large development companies who may have land banks in those areas are likely to wait before developing until the political and economic climate is in their favour. Some participants were cautiously optimistic about the new State government in Queensland flagged its intentions to remove some of the previous government's bureaucratic processes and legislation that hinder development potential. These different responses to regulatory changes demonstrate the heterogeneity within the private property development sector and how particular characteristics of a development firm (e.g., large or small) influence the nature of their response to the risks of climate change.

In summary, the risk of increased flooding due to climate change was not perceived as significant by the participants of this study due to five key factors; development location, the duration of the development process, the uncertainty associated with climate change and associated flood risks, the existing familiarity with flooding, and the current capacity within the industry to deal with severe weather events.

\section{Coastal Storm Surges and Inundation}

Coastal inundation and shoreline erosion pose significant risks to coastal settlements and infrastructure. According to the Australian Government Department of Climate Change and Energy Efficiency's (DCCEE) latest report on Climate Change Risks to Coastal Buildings and Infrastructure; between 196,500 and 288,800 coastal buildings are potentially exposed to inundation and erosion hazards at a sea level rise of 1.1 metre (high end scenario for 2100) ((DCCEE), 2011, pp. 3-9). 
In addition, the report states that between 27,000 and $35,000 \mathrm{~km}$ of roads and rail are potentially exposed to inundation and erosion damage (Figure 4). It evaluates the replacement value of commercial, industrial, road and rail and residential assets at more than $\$ 226$ billion (Department of Climate Change and Energy Efficiency (DCCEE), 2011, p. 3)).

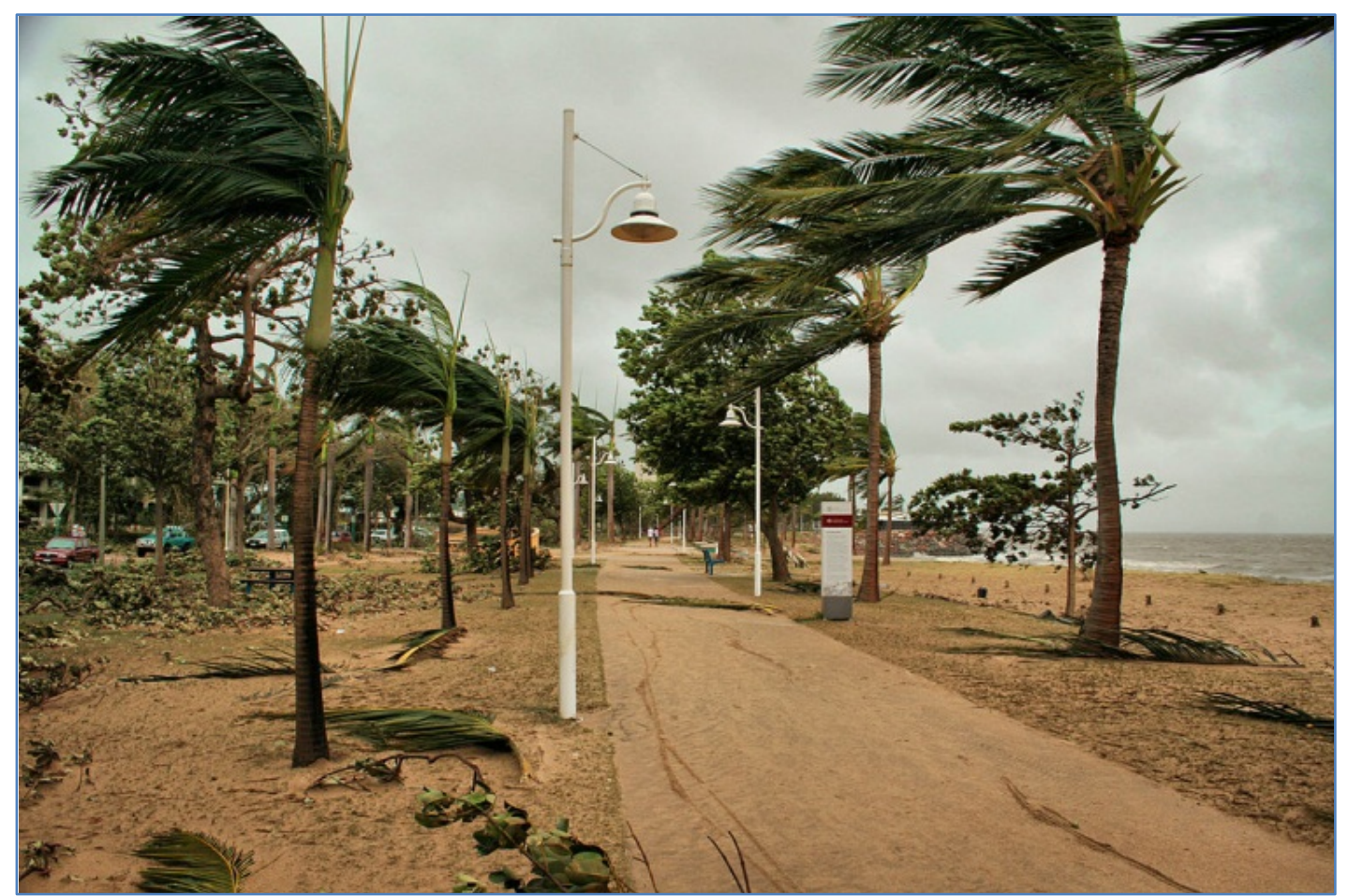

Figure 3 Storm Surge Damage from Cyclone Yasi, Townsville (Source: Rob and Stephanie Levy (2011) via Wikimedia Commons)

As development in coastal areas continues, these costs are expected to grow (CSIRO, 2011; Department of Climate Change, 2009; Wang et al., 2011). Australia's coastal areas continue to experience population growth above the levels of the national average. By 2050 , the population of non-metro coastal areas is forecast to increase by $77 \%$; from 6.9 million in 2010, to 12.2 million (NSCTF 2011, p.6).

Such growth is of concern; despite the increasing pressure to release new land for houses, many coastal Local Government Authorities (LGAs) are yet to develop appropriate policies to restrict development in areas which may be vulnerable to inundation and sea-level rise (National Sea Change Task Force (NSCTF), 2011). Given the potential for growth in market value and future profit; some developers may be tempted to purchase land in the coastal zone, particularly if they have the financial capacity to wait until the economic and regulatory conditions are favourable and supportive of development. This increases the potential risks to property owners and governments from climate change (Department of Climate Change, 2009, p. 136).

To date, little discussion on how these growth trends and projected climatic events could affect the property development sector and the property development process has occurred. According to (2008a, 2008b), however, sea level rise and flooding may impact development at different stages. For example:

- The construction phase could be delayed or temporarily stopped due to flooding, resulting in an increase in costs to the developer.

- Developers in climate vulnerable areas may be challenged by investors, who may choose to 'offload vulnerable assets and invest in climate proofed assets instead' (Bengtsson, 2008a, p.6). 
- Marketing and brand reputation may be damaged if flooding physically impacts the development. In the long-term, failure to consider the potential impacts of climate change across the lifetime of a development could result in a loss of reputation, increased insurance costs, and difficulties in obtaining funding for future developments (Bengtsson 2008a).

In the interviews, developers were asked to comment whether they believed that storm surges and coastal inundation posed a significant risk to their development activities. As with the case of flooding, most developers did not believe that these posed a particular threat to them and their developments. This is mainly because most were not developing on the coast and had a minimal level of exposure to such events. However, even when asked to consider developments in "high risk" locations (e.g., coastal land), most developers still believed that current building standards and regulations would protect these assets from such events and therefore negate the risk of damage.

Furthermore, as with the case of flooding, most participants did not believe that the frequency of these events was high enough for them to be considered a threat to development.

"Most developers would be thinking that they are in and out of there within a time that it is not going to be a problem and even if it is a problem it is not going to be a problem in the time scale over which their development takes place" (CS3, Consultant)."

Interestingly, when asked about storm surges and coastal inundation, most participants kept referring to sea level rise as one of the key "symptoms" of climate change. In many conversations, sea level rise and climate change were mentioned almost interchangeably and without any prompting. A number of participants suggested that this could be the case because governments and the media draw so much emphasis to sea level rise, which is often used as a symbol of climate change. In addition, there seemed to be some level of denial whether storm surges would actually occur.

One participant argued that, given that the combination of conditions necessary for a significant storm surge to occur are so rare, this issue was considered very low risk. The consultant argued that attempts to prohibit or discourage development in vulnerable areas were unrealistic ${ }^{4}$.

"We need a Q100 rainfall event, a highest astronomical tide, and a cyclone all at exactly the same time, which is an extremely rare combination of circumstances...exceptionally rare' (CS9, Consultant).

In addition, many participants commented about the intangibility of concepts such as sea level rise. Sea level rise is one of the more difficult climate change related phenomena to grasp. Unlike floods, bushfires and storms, sea level rise does not occur rapidly and the damage associated with it is often similar to "normal" coastal cyclical climatic variability. Some island nations are already experiencing the negative impacts of sea level rise, but Australia's coastal development has yet to be impacted in any significant way.

One of the participants in the study spent a significant amount of time explaining why, in his opinion, sea level rise may be highly unlikely:

\footnotetext{
${ }^{4}$ Of note, this comment was made prior to the severe storm surge in New York and New Jersey in November 2012 which resulted in a widespread damage to houses and infrastructure
} 
"At the moment sea level rise is not really high enough...the rate of sea level rise around Australia is such that it is hard to get confidence that the predicted sea level rises will occur. We are potentially spending a significant amount of money for a phenomenon that is predicted to occur not for another 40 years" (CS8, Consultant).

This sentiment was echoed by other participants who did not believe that there is 'real evidence' to support the sea level rise theory, and felt it was irrelevant to current developments. The issue of real evidence was raised a number of times, prompting questions about participant's information sources. The majority of developers and planning consultants replied, from <other> consultants, the UDIA and government. On the other hand, sustainability consultants and employees from large engineering firms, sought information from credible scientific sources such as the IPCC, academic peerreviewed publications and other scientific bodies such as the CSIRO.

The vast majority of consultants who were asked whether their clients (i.e. developers) requested any specific information about the topic said that developers were not interested in information about climate change related risks unless they were required by law or other regulations to do something about it. According to one consultant, this lack of interest could stem from the fact that:

"At the moment a lot of the climate projections and sea level rise mapping hasn't been released to the general public, or it is not really available, and there is a lot of restrictions on what mapping is, or is not in, the public domain" (SC1, Sustainability Consultant).

This apparent lack of transparency may influence public opinion, which in turn could shape the way in which developers respond to climate change related risks.

\section{Cyclones}

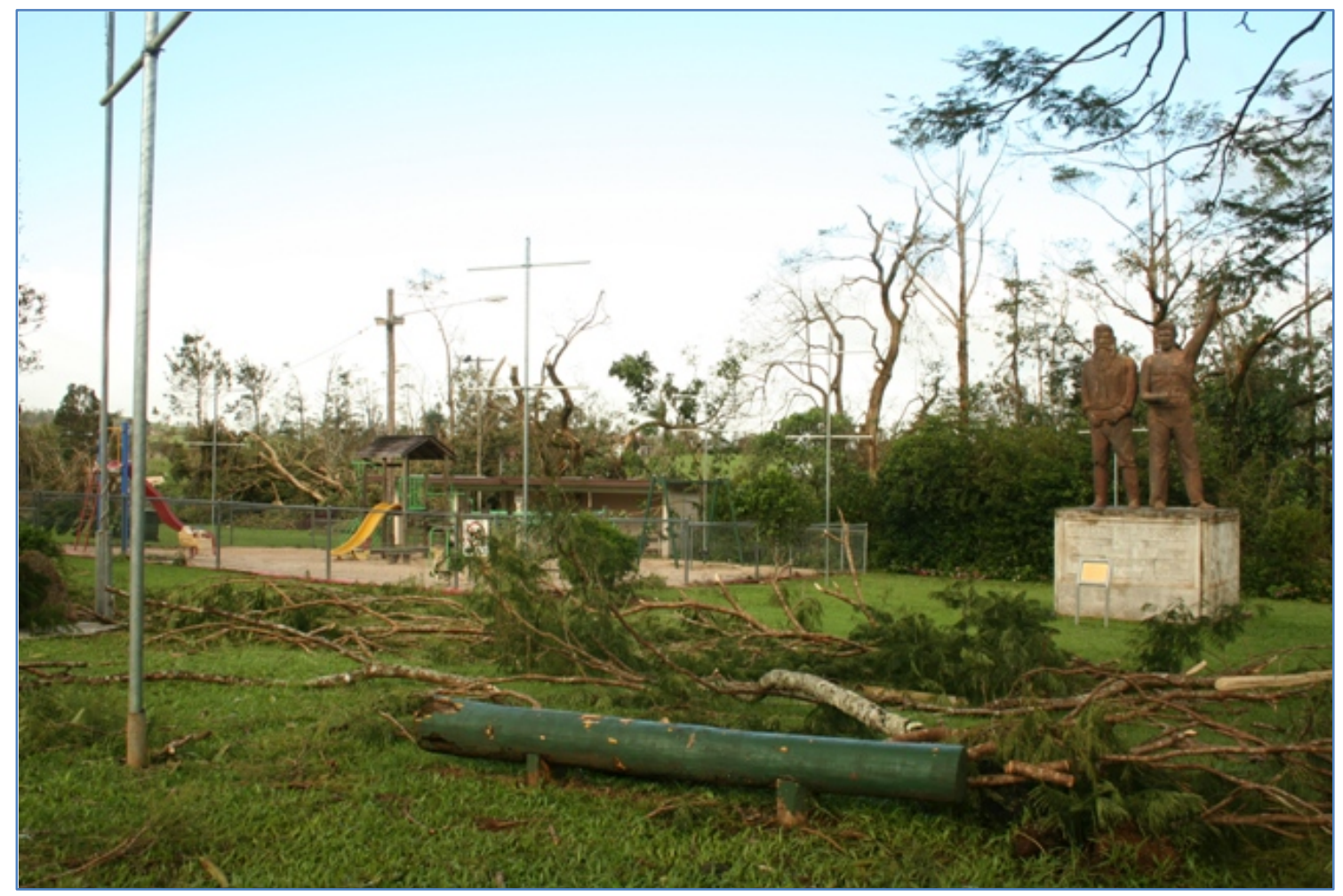

Figure 4 Infrastructure Damage after Cyclone Larry, Mission Beach, 2006 (Source: http://www.rainforest-facts.com/ via Wikimedia Commons) 
The latest predictive climate change weather models for Australia forecast a decreasing trend in the total number of cyclones, but an increasing trend in severity (Bureau of Meteorology (BOM), 2011; Department of Climate Change, 2009). According to the Australian Bureau of Meteorology (BoM), these studies found that severe Category 3-5 cyclones would increase markedly; implying more intense winds and an increase in precipitation levels (Bureau of Meteorology (BOM), 2011).

The major impacts from tropical cyclones and storms result from extreme winds, coastal storm surges and very heavy rain (Snow \& Prasad, 2011, p. 7). Buildings and infrastructure can suffer extensive damage from cyclones and associated high winds. For example, an increase of $25 \%$ in peak gusts results in a $650 \%$ increase in building damage (Insurance Australia group (IAG), 2002)(Figure 5). Many buildings, particularly those built pre 1980, were not designed with appropriate wind loading features, and high winds put greater strain on building material fixtures, claddings and fasteners. Similarly, more intense rainfall increases runoff levels; reduces structural integrity and creates drainage problems and associated flooding (Snow \& Prasad, 2011).

Building codes in Australia have been changed a number of times in different states and territories in response to cyclone damage ( $\mathrm{Li}, 2009)$. For example, following Cyclone Tracy, which hit Darwin in 1974, the NT Government immediately introduced strict wind codes to new and retrofitted buildings. Although these codes were initially accepted, they were soon eroded due to the high costs associated with implementation (Li 2009).

This example raises an important issue, which is the high cost associated with some adaptation measures. Many participants argued that the benefit in going above the specified requirements does not justify the high costs associated with such improvement. In addition, if the changes do not immediately translate into a tangible saving for the consumer, many may be unwilling to pay for it.

More so, with respect to cyclones, SEQ has experienced those in the past and will continue to experience them in the future. However, similarly to the other physical threats, the projected increase in the severity of the cyclones was not viewed by participants as a significant risk to their development activities. One developer felt that even severe cyclone impacts could be readily overcome...if the economic conditions of the region were good. They argued that the responsibility to ensure that developments are protected against such events should lie with the Australian Building Codes Board and not with the developer.

\section{Fire}

Fire weather conditions in Australia are expected to increase as a result of climate change (Hennessy et al. 2005). Research into the potential impact of climate change on fire weather in South-East Australia suggest a significant increase in very high and extreme Fire Danger days by the end of the $21^{\text {st }}$ Century (Hennessy et al., 2005, p. 5).

Fires pose a significant risk to people, buildings and infrastructure. In 2009, Australia experienced what was termed "the country's worst natural disaster"-the "Black Saturday" bushfires in Victoria-which claimed the lives of 173 people and destroyed 2,133 houses (2009 Victorian Bushfire Royal Commission (VBRC), 2010, p.13). This catastrophic event occurred after three consecutive days of temperatures above $43^{\circ} \mathrm{C}$ near Melbourne, Victoria (Figure 6).

Bushfires can cause major damage to buildings. Chen and McAneney (2010) argue that development in fire-prone areas (i.e. adjacent to highly vegetated areas) is one of the key contributors to the increase in fire related home damage in Australia (Chen and McAneney 2010, p. 18). 


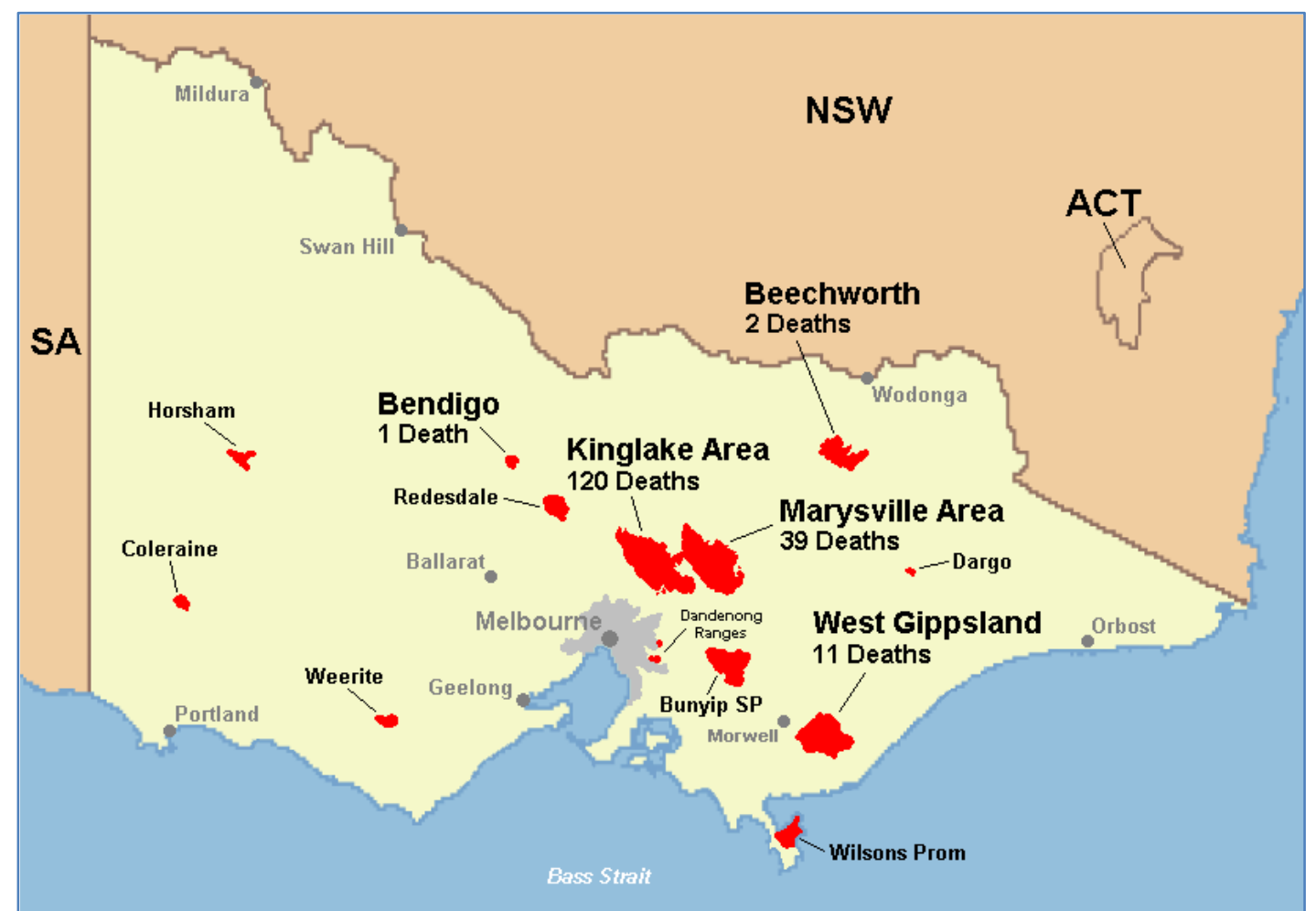

Figure 5 The "Black Saturday" Bushfires, Victoria (2009) (Source: Nick Carson (2009) via Wikimedia Commons)

The Victorian Bushfires Royal Commission (VBRC 2010) reached a similar conclusion. Their recommendations include the introduction of a set of planning and building regulations, aimed at reducing bushfire risks to people and infrastructure (for more information see: VBRC 2010, pp. 31-35).

Most of the participants in this study did not identify fire as a climate change related hazard. This could be because Queensland has experienced unusually wet weather over the past two years due to the La Niña cycle and the memory of bushfires has faded. As the weather once again becomes dry and hot, bushfires may become a significant threat to developments in fire prone areas. Nonetheless, most developers argued that bushfire risks are minimal during the development process. According to the participants, the real risk is not from bushfires occurring during the development phase, but from unexpected regulatory provisions or development restrictions that may arise as governments attempt to mitigate bushfire hazard.

This study found a recurring theme that suggests that developers will only comply with what is required from them by law. This could expose developers, and the residents of their developments, to climate threats in areas where the LGA lacks the capacity to identify climate change related risks and to put in place appropriate measures to minimise potential impacts. This issue could become even more problematic, given that the current Queensland State government is removing some of the previous government's regulatory provisions to address climate change risks. This may leave developers exposed to hazards that they may not be aware of, given that they are largely reliant on government bodies for information. 


\subsubsection{Economic Risks}

This study found that economic issues were the primary risk factor driving the development industry. As one developer stated, "you have got to make a dollar, and it is harder, it is real hard at the moment" (SD2, Small Developer). Factors such as the GFC and the ongoing introduction of planning regulations/restrictions have the potential to compound the economic hardship experienced by many developers. In the context of climate change, economic risks are presented from rising insurance costs, litigation, and more constrained lending conditions. The following sections discuss these risks and the findings from this study.

\section{Insurance}

Weather related insurance claims and subsequent loses have risen dramatically since the 1950s (Mills, Lecomte, \& Peara, 2001 ). Moreover, weather related natural catastrophes $^{5}$ (i.e. drought, flood, storms, cyclones and fire) can result in extremely high costs in human lives and property damage (Figure 7). Globally, over a period of 41 years; $87 \%$ of catastrophe events, $90 \%$ of fatalities, $88 \%$ of overall losses, and $90 \%$ of insured losses, could be attributed to weather related events, with the remainder attributed to geophysical events, such as earthquakes (Figure 7) (Munich RE, 2009).

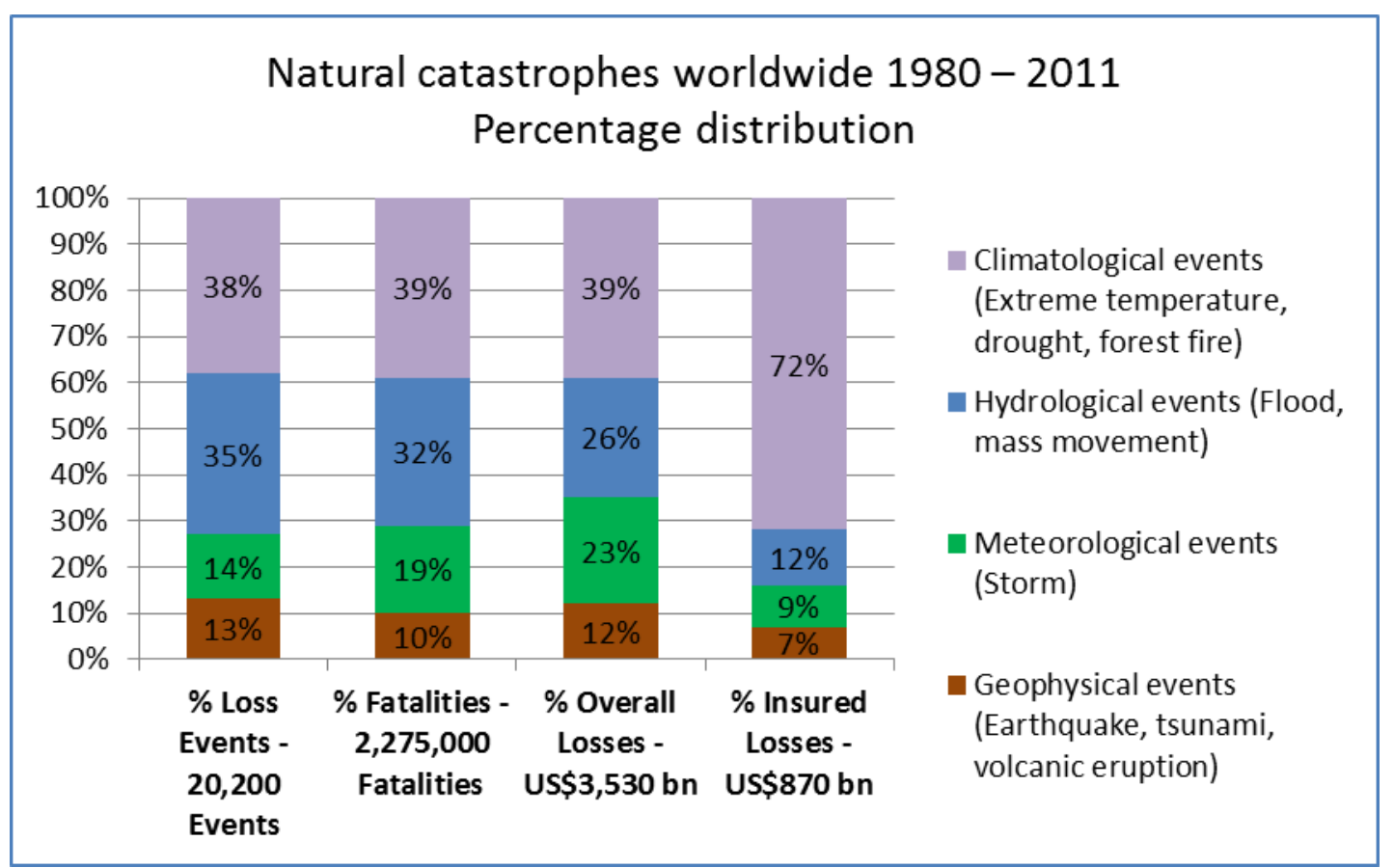

Figure 6. Percentage Losses from Natural Catastrophes (Adapted from Munich $\operatorname{Re} 2012)$

According to Insurance Australia Group (IAG) (2003, p.2), $87 \%$ of insurance losses in Australia between 1960 and 1999, could be attributed to extreme weather events. More recently, a review undertaken by the Insurance Council of Australia found that of the 10 largest catastrophe events in Australia, 9 were weather related (Table 3).

\footnotetext{
${ }^{5}$ Catastrophe events are large natural or man-made disasters causing significant number of claims in a region. (IAC 2011, online).
} 
This rise in weather related insurance losses may be due to two key factors: (1) more people living in high risk areas; and (2) even a small change in a climate factor (e.g. higher temperature) may trigger disproportionate damage to properties and infrastructure (Mills et al., 2001 ) (Table 3).

Table 3 Largest Catastrophe Events in Australia (2011 adjusted \$ amount) Adapted from Insurance Council of Australia (ICA)

\begin{tabular}{l|lll}
\hline Event & Year & State & $\begin{array}{l}\text { Loss (AUD } \\
\text { Millions\$) }\end{array}$ \\
\hline Flood & $2010 / 11$ & Queensland (Qld) & $\$ 2,380 \mathrm{~m}$ \\
Hailstorm & 1999 & New South Wales (NSW) & $\$ 1,700 \mathrm{~m}$ \\
Several Storms & 2007 & NSW & $\$ 1,480 \mathrm{~m}$ \\
Tropical Cyclone Yasi & 2011 & Qld & $\$ 1,352 \mathrm{~m}$ \\
Earthquake & 1989 & NSW & $\$ 862 \mathrm{~m}$ \\
Tropical Cyclone Tracey & 1974 & Northern Territory & $\$ 200 \mathrm{~m}$ \\
Hailstorm & 1985 & Qld & $\$ 180 \mathrm{~m}$ \\
Ash Wednesday Bushfires & 1983 & Victoria/ South Australia & $\$ 176 \mathrm{~m}$ \\
Flood & 1974 & Qld & $\$ 68 \mathrm{~m}$ \\
Tropical Cyclone Madge & 1973 & Qld & $\$ 30 \mathrm{~m}$ \\
\hline
\end{tabular}

It is difficult to establish a direct correlation between the increases in weather related insurance claims and climate change (Bouwer, 2011). However, a growing number of insurance companies are beginning to consider climate change in their risk analyses (Mills, 2009; Ward, Herweijer, Patmore, \& Muir-Wood, 2008). This is because climate change presents significant monetary and reputational risks to the insurance sector, as well as some opportunities (Mills 2009).

Insurers are already responding to the increased risks from severe weather conditions by increasing premiums and in certain areas or market segments (for example, Emerald and Roma which are high flood risk areas), some insurers will not even insure properties, as they believe the risks and costs of doing so are excessive ((IAA). 2011, p.16). At present, properties located in high weather risk areas are paying significantly higher insurance premiums than properties which are located in low risk areas (IAA 2011). The difference in premiums ranges from $\$ 50$ per home in low risk areas to more than $\$ 3,000$ per home in high risk areas (IAA 2011, p.2). The IAA (2011) warns that insurance premiums could more than double in response to climate change and associated extreme weather events.

However, despite the floods of 2010/2011, there is little evidence that SEQ insurers are raising their premiums or implementing any other measures to respond to potential climate change. Most SEQ respondents reported no premium rises or other insurance issues. However, in North Queensland (Maryborough to Cairns), insurance premiums have risen significantly, particularly for strata and group title complexes. In some cases, insurance premiums have risen by over $300 \%$ (Figure 8 ). These price increases began after Cyclone Larry (March 2006) and major storms in Mackay in 2008, but increased more rapidly after Cyclone Yasi (January/February 2011).

More recently, floods have again occurred in Queensland (January 2013). These floods are too recent for the costs to be quantified, or for the possible response of the insurance sector. However, it is highly likely that premiums in flood affected areas (particularly those repeatedly affected), will rise significantly (Thompson, 2013). 

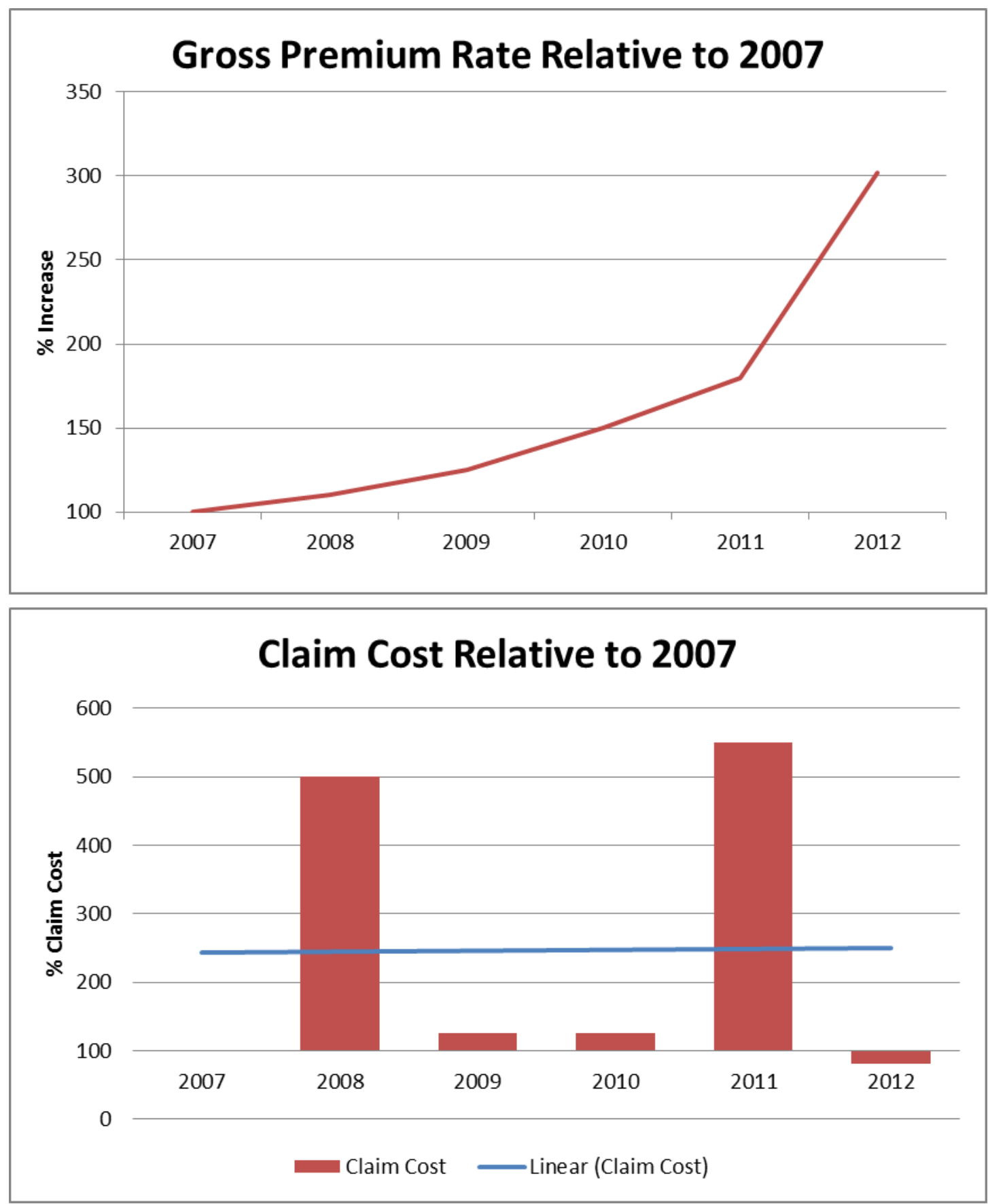

Figure 7. Rise in North Queensland Strata insurance premiums since 2007 (Adapted from Australian Government Actuary, 2012)

So steep was the increase in premiums, that the local Federal Government Minister instituted an enquiry into the issue, one result of which was a major report by the Australian Government Actuary (2012). The report concluded that the premium rises were justified, given that the small number of strata title insurers in North Queensland, recent rises in catastrophe reinsurance rates, and previous under-pricing of insurance.

However, these premium rises have had significant negative impacts on the property development industry in many North Queensland towns, exacerbated by the GFC and the collapse of a large investment company (Storm Financial). For example, unit prices in some areas have reduced by over $200 \%$ and a number of large developers have gone bankrupt. Despite the dramatic reduction in prices, some buildings still have over 
$50 \%$ unsold stock, because the Body Corporate fees (including insurance premiums) are almost equivalent to the interest payment on the mortgages (Studdert, 2012).

While this issue has not yet impacted SEQ, there is high level discussion amongst reinsurers on the potential risk of weather catastrophes to the region. Some insurers are reportedly considering doing further risk assessments and increasing premiums based on climate change risk, but have not yet signalled any intentions to the market.

The survey and subsequent discussions with developers and allied professionals suggests that, at present, insurers are not signalling any intentions to change existing insurance policies for the SEQ building and property development sector in response to climate change. However, most participants acknowledged that there are some discussions with respect to climate change in the higher levels of the insurance industry, and that it is possible that this could become a future problem to their firms.

As insurers continue to carefully inspect the damage from severe weather events in Australia and abroad, and as information about climate projections becomes more consistent, it is likely that premiums will continue to increase. This could have an indirect effect on developers who might find that their prospective target market is more cautious about purchasing properties in areas that are prone to severe weather events, flooding and bushfires. As in North Queensland, some developers may be left with unsaleable housing stock, or stock that is particularly difficult to sell, which in turn could impact their ability to secure funding for future development.

When asked whether they felt that increasing premiums would affect their target markets, most developers replied that people don't seem to be bothered about it, and that other, more important factors, such as the price and location of a property influence purchasing decisions. Interestingly, they believe this is likely to change in future. At present, increasing premiums are only being felt in specific localities, but as the area impacted by severe weather events expands, the signals about potential climate change risks are likely to strengthen and filter down to the consumer and subsequently to developers.

Developers also did not express concern about the possibility that insurance companies would refuse to insure developments in risk prone localities, such as coastal areas or fire prone areas. Many respondents believed that lending institutions, consultants, LGAs and builders would be more impacted by such changes to insurance policy, than developers.

\section{Litigation}

In recent years, a growing number of climate change related legal disputes between developers and local LGAs have occurred (Briggs, Taberner, Voros, \& Bilski, 2010; Fitzgibbon, 2010). Most disputes were in relation to development in coastal areas where the risk of flooding or inundation due to sea level rise was deemed as too high (Freehills, 2010).

The UDIA has acknowledged the growing risks of litigation for property developers as a result of climate change (Urban Development Institute of Australia (UDIA), 2008). It argues that developers need to be aware of the potential legal consequences for developments in areas that are vulnerable to climate change hazards (e.g. floods, sealevel rise and cyclones).

However, as with the case of insurance, developers do not believe that the risk of litigation lies with them. Instead, they believe that those who develop and enforce regulations, i.e. government, will mostly be exposed to litigation risks if things go wrong. To date, most legal disputes with respect to climate change are associated with an appeal against decisions by LGAs to prohibit development on a particular site. There have yet to be any cases where developers have been sued by the public for negligence with respect to climate change. In a sense, because developers always 
comply with what is required by law, the risk of litigation is minimal and is transferable to those who approved the development in the first place (e.g. local government or affiliated agencies).

However, litigation risks may derive from unexpected sources, such as shareholders (Guzy, 2007). According to two participants, both consultants, company directors are required by law to disclose any information with respect to known risks. A failure to disclose known risks is a legal offence as stipulated in the Corporations Act 2001. This requirement may put developers, mainly large scale developers, in a position whereby they could be challenged in court for failing to protect their shareholders' interests with respect to climate change risks. However, developers may be able to argue that climate change risks do not fall within the categorisation of "known risks" as there is too much uncertainty surrounding them. Nonetheless, if a company's reputation is compromised due to a failure to consider the latest evidence about climate change related risks, there could be a scope for a legal challenge ${ }^{6}$.

\section{Lending}

Most developers relied on external sources of finance to carry out their developments. Property finance may be obtained from a range of sources, but most commonly from banks. In Queensland, developers tended to use two key banking institutions, namely: ANZ and the Westpac Group (which includes St. George Bank), and to lesser extent, the National Australian Bank (NAB). During the GFC period, banks significantly restricted their lending conditions, with the result that local lending opportunities were severely curtailed, and some developers sought alternative sources of finance, such as foreign investors (mainly from Asian countries) and joint ventures (Ashe Morgan Winthrop, 2011; Coiacetto, 2012, pp. 56-8). The restrictive conditions that have characterised property lending over the past three years have resulted in a greater scrutiny over the nature of the proposed development and associated risks.

Climate change could potentially present a number of indirect risks to developers who seek external property finance. Lenders, naturally, would like to ensure that their capital is invested in the least risky way. Property developers who intend to develop land located in hazardous areas (e.g., flood or cyclone prone, etc.) are required to provide all the necessary information to demonstrate that the proposed project is not likely to be negatively impacted by such events. Financiers interviewed in this study required that developers obtain all the necessary approvals for their development prior to seeking finance. This means that if there are any regulatory restrictions associated with the site, the lending institution will be aware of them well in advance, and is most likely to refuse the application; as indicated by one of the interviewees, "we take little or no planning risk...we won't fund a site that doesn't have appropriate approvals" (FI1, Financier) ${ }^{7}$.

Discussions with representatives from two large banking institutions reveals that at present, banks do not consider climate change per se as a significant risk for development. Climate change related physical and regulatory risks simply fit under a risk categorisation framework. However, location of development is one of the key factors that banks consider when assessing a loan application. Nonetheless, while some coastal properties, for example, may be deemed as highly vulnerable to cyclones, storm surges and floods, that same location might provide good views and would appeal to the higher end of the market, thereby, promising a good return for

\footnotetext{
${ }^{6}$ This point has yet to be tested in court.

${ }^{7}$ Of note, this is more relevant to Australia than other countries where lending has been less risk averse.
} 
investors. In that case, the financing institution will use a number of factors to assess the level of risk.

One of these factors is the duration of the loan, which is rarely more than 2 years. This is perhaps one of the key aspects that influence financiers approach to climate change related risks. The risk calculation is based on the likelihood that a particular event will occur with the duration of the loan, which in most cases is unlikely. According to one participant:

"If it was significantly flood prone, from normal seasonal type of stuff, you almost certainly wouldn't get approval - the developer wouldn't receive approval or we would have a strong view that we might let that one pass. If it is a 1 in 25/50/100 (risk of flood) and we think that we are going to be out of it in 18 months, the chances are that we will just go and do it" (FI1, Financier).

Banks, like developers, rely heavily on information provided to them by external consultants. This strongly influences the degree of scrutiny used with respect to climate change. Once again, the quality of the information provided by the consulting entity will greatly influence the degree of scrutiny employed by the lender when it comes to assessing climate change related risks. Consideration of climate change is therefore given only as far as approvals are obtained from the relevant agencies and all the appropriate boxes were ticked.
"We look at maybe a little shortish to medium situation here and saying is this site fully approved by all levels, because there are plenty of referral agencies, and if the answer is yes, and we have a client that knows how to do it, and we have got somebody to build it, and we have sold a whole lot of that product, then we are going to be fine with it" (FI1, Financier).

Therefore, the impact for developers seeking to obtain finance lies predominately with the costs associated with gaining the appropriate approvals. In cases where technical challenges are presented (e.g., difficult slope, water management on site, etc.), developers are required to employ professionals, approved by the lending institution, to minimise potential complications. This is likely to incur additional costs and could make high risk developments non-profitable. In addition, as banks require a high level of competency from the development team, and as lenders appear to be uncompromising when it comes to planning and regulatory risks, this might provide a 'natural protection' barrier from some climate change related risks.

\subsubsection{Social Risks}

\section{Market risks}

Market risk is arguably one of the most important risks in development. It is often realised at the end of the project, when the investment of substantial finance and resources has been committed. To some extent, the GFC changed the timing of market risk, as financiers are insisting on high levels of pre-sales before they will consider funding a development. Other risks, such as planning risk, usually occur earlier in the project, when considerably less has been placed at-risk financially.

The development task is to accurately target the relevant market or submarket and to create the right products for that market. Markets present considerable risk and uncertainty. Demand and supply can both be volatile and difficult to predict. Developments have long gestation periods and that creates timing problems. The developer's challenge is not merely targeting a market opportunity that exists in the present but rather one that the developer anticipates will exist at some time in the 
future, when the development products come on stream (notwithstanding some capacity for pre-sales).

Developers require certain skills and competitive strategies to operate in certain markets (and this creates a friction against switching to other markets and opportunities that might emerge). Some developers try to specialize in certain markets to compete and thus create a niche for themselves. They may over time evolve an organization, staff and skillset to effectively supply and compete in specific markets and that helps define but also constrains their development activities.

Climate change is likely to affect market change through the potential modification of consumer preferences (e.g. for greener developments), demographic change and so on. Also, particular market sectors and segments may be more vulnerable to the physical impacts of climate change than others. For example, industrial property is often on level and low-lying land, possible at risk of flooding. In residential segments, the lower and upper end lifestyle markets may both be at risk, for example on coastal and/or riverfront locations.

However, consumer preferences for greener development have stalled over the past few years, possibly as a result of the GFC. According to most of the participants, there is no current demand for climate resilient development, particularly if it comes at additional cost. This 'lack of interest' in addressing climate change risks may be the result of a number of factors. First, the "appetite" for green or sustainable development is directly linked to economic conditions and consumers will respond negatively in times of economic hardship. Second, some participants argued that people do not necessarily comprehend what climate change could mean to them and how that relates to the house they may choose to buy.

"The change is slow and long term. I mean, for me I will be dead before the major changes are evident. I think most people say, well that's not going to be my problem today. I have got bigger issues to deal with today. Food, petrol for my car, kids at school' (CS1, Consultant).

While climate change is generally viewed as occurring in the future, other issues such as rising costs of electricity and water, do influence the choices people make with respect to their homes. Many participants said that from their experience, people are willing to participate in conserving energy or water, which are tangible issues that have been addressed by governments in the form of incentives such as rebates for rainwater tanks, solar hot water systems and PV solar panels for generating electricity.

Although there appears to be a decline in the demand for green products, there is some indication that many developers do continue to seek green or sustainability accreditation (e.g., EnviroDevelopment). However, such schemes do not use climate change as their 'catch phrase', but rather the term 'sustainability' to ensure that the message transmitted to the consumer is one of "feel good" and hope. Consumers' preferences have a direct impact on the way developers design and build their product. Many developers and consultants have acknowledged how the 2011 Brisbane floods changed the way properties are built in flood prone areas.

According to one consultant: "There are some interesting interactions there between the market wanting something and developers wanting to respond to that in a really positive way. I was just reading yesterday about a developer in the Gold Coast on the floodplain, and he has all these conditions like the body corporate will have a boat and a trailer, and a helicopter pad on top of the tower, and an early warning system to their residents" (CS5, Consultant). 
Similarly, another respondent said: "We are seeing services elevated beyond even the first floor. Put up on the roof or the second to top floor. We are seeing a lot more understanding of Q100 levels and the importance of having a setback from even those levels and integrated Water Sensitive Urban Design, in parks and open spaces and low-lying areas. And yes, that is a response to actual events, but it is also a response, again, to the consumer, or the tenant saying if that happens again I don't want to lose my staff and my operations for six weeks" (IN1, Industry Body Representative).

These comments indicate that developers are incrementally adapting to changes in consumers' preferences by modifying the design of their products. In the context of climate change, this could mean that, as people become more aware of the link between climate change and severe weather events, the demand for climate resilient housing will rise and the development sector will respond appropriately. However, it is yet to be clear which developers have the capacity to respond to changes in consumers preferences. This possibly depends on their access to resources, information and skills. More discussion on adaptive capacity is found in Chapter Seven, The Adaptive Capacity of the Private Urban Development Sector.

\section{Reputation and Legacy risks}

Maintaining or enhancing corporate reputation is one of the primary goals of any business. However, to what degree climate change is perceived as a factor that could influence a business' reputation is a matter that varies from one firm to the next. A survey conducted by the Institute of Sustainable Enterprises in Michigan University explored the key factors that motivated businesses to address climate change risks. The results of this survey revealed that the third most important motivator was to 'Enhance Corporate Reputation' (Hoffmann, Worthington, \& Higgs, 2006). This suggest that the business community recognises the benefits associated with addressing climate change issues, or alternatively, can see its potential to damage their reputation if they fail to do so.

The findings from this study reflect a similar sentiment, albeit not necessarily in the immediate context of climate change. Reputation and legacy issues were raised many times by the participants in the context of sustainable development. As a whole, the development sector apparently seeks to be viewed positively by the public, as seriously considering the well-being of future generations.

Many developers and consultants argued that the development sector is becoming more responsive to issues such as water and energy conservation. However, there seems to be a strong link between economic conditions and the willingness to address sustainability issues; the better the economic conditions, the more developers were willing to engage in efforts to incorporate green and sustainable principles.

Although both large and small developers viewed reputation as an important factor, the larger developers seemed to be more committed to 'doing something about it'. This is not surprising given the high costs associated with implementing sustainability measures, and that larger developers usually have the financial capacity to access resources and engage appropriate professionals (e.g., sustainability and climate change consultants). Nonetheless, it is not to say that smaller developers were not actively pursuing ways to enhance the sustainable nature of their developments. The level, however, to which such efforts were implemented, often depended on the target market and its interest in such schemes.

"Big companies are very concerned about the enduring value of their brand, and their position as a developer. They want to show long term value. How do you build that legacy? That reputation?" (AR2, Architect). 
Another point of difference between smaller and larger developers was with respect to the ongoing commitment that they have towards their product. Large developers tend to have lengthier time commitments, and therefore, their reputation may be tested over a longer time scale. On the other hand, smaller developers may have a very short term commitment to their product, but their overall brand may be damaged if they fail to provide good and reputable standards and product quality. With respect to climate change risks, the larger developers who tend to remain involved for longer periods of time, have a stronger incentive to consider potential risks simply because these are more likely to eventuate while they are still involved in the development. For the smaller developer, this is unlikely to be the case.

"Legacy is critical for those sorts of companies. These are publically invested companies. Their reputation, it is critical to them. You look at $<$ large developer> advertising itself with its master communities where it's still staying and managing a lot of them. Its critical to them. Absolutely. Because it impacts on their next sale" (CS1, Consultant).

To sum up, reputation and legacy are issues that were raised by many participants regardless of the size of the firm or the type of product offered. Nonetheless, there were degrees of difference in the context of climate change; mainly due to the time scale of development and the overall level of involvement and commitment of the company post development.

\section{Timing and Uncertainty}

Some issues raised in this study can be grouped to a number of distinct themes, as they seemed to repeatedly occur in different contexts. These themes were; the way in which timing of development influenced participants response or attitude towards climate change related risks, uncertainly of climate change, and the intangibility of some climate change related risks such as sea level rise and storm surges.

The short term commitment that most developers have to a site or project has implications for their attitude and perceptions of climate change. The key implication of this short term commitment is that physical climate events pose a relatively low risk to the developer; for example, the risk of flooding or other severe weather events during the actual development phase is likely to be fairly low because this phase may only last 1-2 years. For example, a Q100 flood has a probability of occurring once in a hundred years. Although, of course, such floods can, and do, occur more frequently, the short duration of the development phase does remove some risk from developers, leaving the bulk of the physical risk with the eventual users, the community, the insurers, and the government.

Timing is therefore one of the key factors that influences how developers felt about climate change risks to their projects. As mentioned above, developers aim to get in and out as quickly as is feasible mainly due to financial considerations. However, developers are liable for damages for seven years after completing a development; but once again, this liability might be transferred to builders, insurers or even the approving agencies.

While physical risks associated with climate change were not perceived as significant or even real by most of the participants, regulatory risks were taken seriously. There was a strong negative sentiment towards government interventions with respect to climate change adaptation, and more specifically, to the Coastal Plan, which aimed to restrict development in certain coastal areas. During interviews and focus groups, there were often strong negative comments and high levels of dissatisfaction with the principles underpinning the Coastal Plan and the processes that preceded its release. Further investigation into the sources of these sentiments suggests that it partly stems from the way these respondents perceive climate change and its associated risks. 
Although the Coastal Plan aimed to protect future development from floods and sea level rise, participants did not believe that these risks were significant enough or even real, and therefore, were not convinced by this justification.

Participants argued that the science about climate change is ever-changing and that there is still much uncertainly with respect to the projections:

"Government is saying at the moment that cyclones might become $10 \%$ more intense, severe rainfall intensities might increase by $10 \%$ and climate change sea level rise might occur. I don't know if there is a lot of science behind those $10 \%$ changes, they seem to be a reaction that simply says we need to do something right now. So let's make it $10 \% "$ (CS8, Consultant).

This lack of clarity on behalf of government may have influenced why many participants heavily criticised regulatory attempts to address climate change.

Interestingly, while some participants thought that the science about climate change is uncertain and that government is overreacting, some participants (mainly sustainability consultants) argued that government is using very conservative models of climate projections and is failing to provide accurate and up to date data to its constituents and those who operate within its boundaries (e.g., developers).

\begin{abstract}
"Politicians run around saying we are doing the right thing but when it gets down to the actual imposition of conditions and legislation, the details are not there at the moment, and governments take a quite conservative view about the impacts" (CS8, Consultant).
\end{abstract}

Further, many LGAs may not have the capacity to investigate climate change risks in their local context, and the regional, state or national based studies may fail to identify those risks.

The implications of these scenarios can be far reaching with respect to developers' ability to respond to climate change, that is, their adaptive capacity. If developers rely on information provided to them by government, insurers or other regulatory bodies (e.g., the Australian Building Codes Board (ABCB)) with respect to climate change risks, they may underestimate the potential for those risks to occur. This lack of awareness could in turn negatively influence their capacity to adapt to climate change and in some cases lead to maladaptation.

\title{
Tangibility issues
}

Perceptions towards climate change are also influenced by the public media, and how it communicates the threats of climate change.

"There is a real communication problem around climate change. Every time on the news we talk about climate change, there is a picture of a melting ice cap and a polar bear. That is what people think is climate change. That is what I thought of as climate change until I go no, you are supposed to know more about this and break it down. So I think if you said to people, are you worried about bushfire risk with your projects you get a different response. So l'm almost at the point where we need to stop talking about climate change" (IN1, Industry Body Representative).

This statement reflects the majority of the participants' opinions about climate change. In many of the interviews, climate change was used almost interchangeably with sea level rise and many participants "complained" about the intangibility of climate change and argued that they cannot take climate change related risks seriously because it was difficult to observe any evidence of sea level rise or increased severe weather events in Australia. However, when the risks were broken down into familiar concepts such as 
flooding and bushfire, most participants expressed some level of understanding and appreciation of the potential damage that can be caused by such events. Nonetheless, when it came to developers actually addressing these issues, they relied almost solely on the regulatory requirements of government.

In conclusion, the physical risks of climate change were not perceived seriously by most developers and consultants, primarily because of a lack of belief that major climate events would occur during the development process. Therefore, climate change, if considered at all, was just part of a myriad of development related risks and not a significant issue that required any additional consideration.

On the other hand, there was more concern with regards to indirect climate change risks such as changing regulations and consumer preferences. Developers and consultants repeatedly complained about the lack of certainty from government with respect to development approvals and the added complexity that environmental issues, including climate change, bring to the system.

"If we took the biggest extreme example of it and attempted to adapt to it, then where do we do actually development? Because there is risk all over the place. If drought is one of your climate risks, maybe Australia should be left alone. What I am saying is that you can adapt anywhere in that thing, but somewhere we need to draw a line and say: this is where we are with climate change" (CS3, Consultant).

\subsection{Conclusions}

Climate change poses a range of direct and indirect risks to property developers and allied industries, such as consultants and financiers. Direct risks include physical risks (i.e. floods and cyclones); and indirect risks include economic risks (i.e. financing and insurance), institutional risks (i.e. regulation, policy and industry structure), and social risks (i.e. green demand and corporate reputation).

Direct, physical risks were not seen as particularly important. Survey respondents ranked very few risks above 3 (neutral). Flooding, cyclones and extreme rainfall ranked higher than other physical risks, and are likely indicative of the recent Brisbane floods (2010/2011) and Cyclone Yasi (2011). When differentiating by category, larger developers and consultants tended to view physical risks as more important than did small and medium developers. Most small developers ranked physical risks as unimportant.

Likewise, few interviewees or focus group attendees viewed physical risks as particularly important. The only exceptions to this were large developers and specialist consultants (i.e. sustainability consultants). This is likely to represent the different spatiotemporal scales of developments; smaller developers aimed to be "in and out as quickly as possible", whereas bigger developers had a longer exposure with much larger projects.

Developers and consultants were very aware of flooding, probably because of the 2010/2011 Brisbane floods. However, they felt that their exposure to risk could be managed by adhering to government regulations and the $\mathrm{BCA}$, and with insurance. It was noted that severe weather events, such as major floods or cyclones, had the capacity to rapidly change the BCA to adapt to future such events.

As with flooding, most respondents did not consider that storm surges and inundation would pose any significant threat to their business. Many also felt that storm surges were not a major issue because they did not develop in coastal areas. One respondent in a low-lying coastal area felt storm surge was not a threat because it would require an 
extremely "rare combination" of a Highest Astronomical Tide (HAT), cyclone and a significant rainfall event.

Of note, many respondents when asked about storm surges, referred to sea level rise; considering this as the primary impact of climate change. These respondents felt that sea level rise was only projected to be a major concern in "90 years' time" and thus was not relevant to them. They also expressed scepticism about the likelihood of sea level rise occurring at all, often referring to personal experience, and past weather trends.

Interestingly, when the interviewers mentioned other potential impacts of climate change, such as cyclones tracking further south, then respondents felt they would pose a high risk to their business. This highlights a potentially important communication and information gap; in that the media often portrays climate change in terms of melting icecaps and rising sea levels, and not the entire gamut of changes projected to occur.

Few respondents specifically mentioned or even considered that other physical risks, such as bushfire, were of importance. This is unsurprising, as most participants were based in SEQ, generally not a high risk bushfire area. Most respondents considered that any such risk would be covered by LGA planning codes.

Unlike physical risk, indirect risks were viewed as significant by survey respondents. Of these, institutional risks were the most important; and the major risks were seen as: more lengthy approval times; more onerous government charges; and regulatory risk. The second highest ranked risk category was economic risks, and of those; changed market demand, higher government charges, and changes in valuation; were considered the most important risks. Of note, these categories are not discrete, and there is some overlap between them; for example, many risks can be considered both institutional and economic (i.e. lengthy approval times can lead to cost overruns). These risks are discussed further in Chapters 4, 5 and 6.

Like survey respondents, interview and focus group participants also viewed economic and financial risks as extremely important. Developers and consultants felt that the GFC had severely impacted their business and said that many firms had gone bankrupt or exited the market. This had had a flow-on effect on bank lending, and all participants reported that it was extremely difficult to obtain bank finance. The issue was exacerbated by the regulatory environment, which was seen as unnecessarily delaying development approvals, thus costing money.

The insurance industry has the potential to be a major driver of climate change adaptation, by increasing premiums in high risk areas, or requiring adaptive measures in exchange for reduced premiums. Further, it is an industry that is exposed to very high risk from climate events. However, despite the Brisbane floods, premiums had not risen, at least in SEQ. This was not the case in North Queensland, where some premiums had risen by over $300 \%$ in the aftermath of the 2008 Mackay storms and 2011 Cyclone Yasi.

The threat of litigation (i.e. if a development is flooded) could also be seen as a climate risk. However, this was not seen as posing any significant risk to developers or consultants. Respondents felt that if they adhered to the building codes, government regulation and planning requirements, and often, their own code of conduct, they would be protected from any litigation. Litigation was more often used to counter adaptive measures; i.e. by developers suing local governments that wished to implement planned retreat (i.e. Belongil Beach in the Byron Shire Council).

Lending institutions can potentially pose a significant risk for developers, if they refuse loans on properties subject to high levels of climate risk. Although bankers were very conscious of site risks, such as flooding, they generally assumed that these had been dealt with at the development approval stage (and they would not lend money without 
an approval in place). No respondents or bankers reported that climate change was an important criterion when assessing loans.

Further, banks might be more willing to lend money on some high risk areas, such as waterfront properties, if all approvals were in place, and the development had a high proportion of pre-sales. In addition, the short time frame of most development loans (under two years) meant that banks had a relatively low exposure to climate risk. Banks also relied heavily on information provided by the regulatory agencies and consultants, but this information was not necessarily comprehensive or accurate.

Market risks are arguably the most important risk faced by the developer because they occur at all stages of the development. Pre GFC, most market risk was found at the end of the development, after the developer had invested significant money and resources into the project. However, with the requirement by bankers of a high proportion of pre-sales, the timing of market risk had shifted somewhat.

Market demand is relatively fickle and can change rapidly. Therefore, risk could manifest in an increased consumer demand for "greener" properties. Because property development is a lengthy process, "older" style developments may not sell due to changed demand. At present, no demand for green products was evident in the residential market, which was principally price conscious. However, there was demand for sustainable, energy efficient products in the commercial and retail sector, and to some extent, in the retirement market.

Attitude to climate risk differed widely amongst participants, ranging from some who denied it existed, or that it was caused by man; to consultants specialising in climate change adaptation. To a large extent, attitude is based on knowledge, and the information used to acquire that knowledge. Most survey respondents considered they had good to high degree of knowledge about climate change. However, the more qualitative process of interviews and focus groups identified that this perception was inaccurate. Many respondents did not have a good grasp of climate change, were often sceptical, and often equated it only with sea level rise.

To a great extent, the general lack of concern about physical risks could be attributed to the timing of development. As most developers wanted to be "in and out as quickly as possible" (and the banks would only lend money in the short term), they considered physical risk too distant, or too far in the future to be an issue. If a climate event did happen during the development process, most respondents said that their insurance policies would cover it.

Complicating the timing issue, was uncertainty over climate change. If developers and consultants are sceptical about climate change, and they saw it only happening in the distant future, if at all, they were not likely to consider it much of a risk. Moreover, many respondents appeared confused about the complexity and intangibility of climate change. This was largely related to their attitude, and their sources of information. Climate change was also deemed to be overly politicised, and a loaded term. 


\section{HOW INSTITUTIONAL STRUCTURE AND THE DEVELOPMENT PROCESS INFLUENCES CLIMATE CHANGE ADAPTATION}

\subsection{Introduction}

The development industry is complex, heterogeneous and dynamic. Arguably, every developer is different, and is subject to differing levels of governance, depending on the company structure and operating strategies (Adams, Croudace, \& Tiesdell, 2012; Coiacetto, 2001, 2007, 2009). Moreover, the type and scale of development, as well as the development process, largely depends on the structure of the developer (Coiacetto, 2007, 2009).

To approach the problem of climate change adaptation in the development industry, it is vital to first acknowledge these differences (Coiacetto 2001; 2007). It is important to identify the developer, to identify how the challenges and issues of climate change adaptation will influence the different participants in the industry.

Different developers have different exposure to the direct risks of climate change; particularly with regard to the duration of a developer's commitment to a site or project relative to the life of the built environment. Further, project commitments fall along a temporal continuum; from the very short term (taking a year to plan, construct and sell), to those which last for many years, if not decades.

This study found that the institutional structure and operating strategies of SEQ property developers differed widely, along both spatial and temporal scales. Developers ranged from large multinational companies with multiple developments around Australia, to very small firms, engaged only in one development. The developments themselves were extremely diverse; from thousands of lots in masterplanned communities, to high rise, multi-use buildings in the CBD, to "mum and dad" duplex sites. The duration of development works ranged from less than one year to 20 years or more.

In addition to the actual developers, the development industry engages a wide range of sub-contractors, architects, builders, consultants, and other specialists. Some firms only use sub-contractors, some only employ in-house staff, and some use a combination of in-house staff and sub-contractors. A common type of sub-contractor is a consultant, who navigates the complex regulatory environment, by preparing development applications, sometimes even lobbying politicians for legislative change.

This chapter details the findings of the research; the diverse nature of the property development sector; the structure of developers, the development process and some development types. It also describes the use of allied professionals, the subcontractors and consultants. The work is informed by the results and findings of the research, as described in Chapter 2 (Research Activities and Methods), with statistics on development firms, and quotes to add deeper layers of meaning to the quantitative results.

\subsection{Developers General}

\subsubsection{Developer structure}

There is no one type of property developer, nor do they practice the same operational strategies (Coiacetto, 2007, 2009). However, when considering the capacity of developers to adapt to climate change, it is important to consider who the developer is, and more importantly, their operational strategies. Identifying the developer can establish how the challenges and issues of climate change adaptation will influence the different participants in the industry.

42 The capacities of private developers in urban climate change adaptation 
The internal structure of development firms or development sections within larger corporations can differ greatly. For example, a large scale developer may be an individual who relies mostly on consultants and contractors, or a large organization employing a large number of in-house staff (Coiacetto, 2009). This internal organisation influences the degree of control over the development process and the quality of the final product; the firm's capacity to tailor its product to the market and to adjust supply to changing and emerging circumstances (Coiacetto, 2007). Further, the size of a development company can directly influence not only a developer's operational strategies, but other factors, such as exposure to risk, lending ability and type of development products.

A diverse range of SEQ developers and allied professionals (a range of different consultants, architects, solicitors, and State and Local government staff) participated in this study. This diversity was directly related to the geographic area, type of developer and development, and process and timescale of developments. Moreover, this diversity directly influenced developer's ability and willingness to adapt to climate change. The size of a developer, and the spatial and temporal scale of their developments, were the most significant factors determining whether the respondents felt climate change posed a major risk to them.

The majority who answered the survey question on category of respondent (41 out of 45 , or $91 \%$ ) represented small to medium sized firms, with turnovers under $\$ 20$ million dollars. Of those with turnovers in excess of $\$ 20$ million, five were listed on the Australian Stock Exchange (ASX). Two participants were State Government developers; semi-independent bodies, managed as corporates, but funded in part by the Queensland Government ${ }^{8}$ (Figure 9 ). The numbers of staff directly involved in property development differed widely. In the survey, most firms were small, with fewer than 5 staff (38\%), but approximately $11 \%$ had greater than 200 staff, with the same number listed on the ASX ${ }^{9}$. Nearly all development sizes were larger than 50 lots or homes $(56.1 \%)$, and $38.6 \%$ of firms had been in business for longer than 20 years.

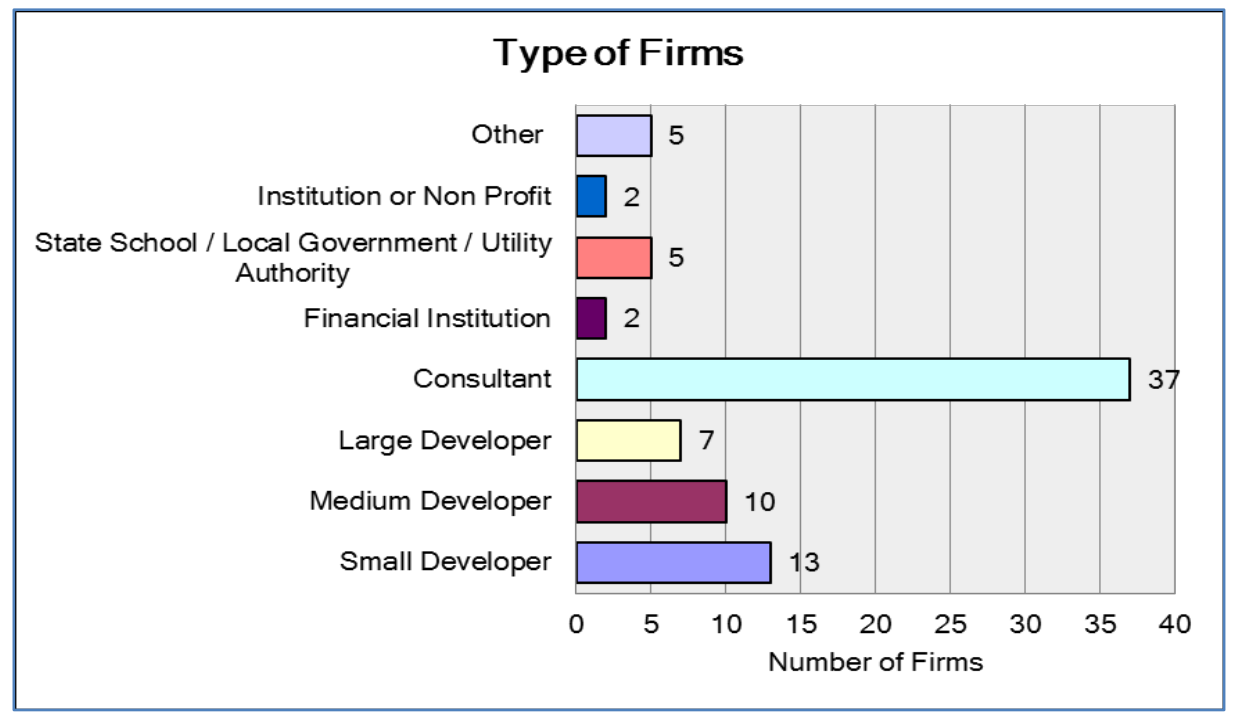

Figure 8 Number and Type of Firms Participating in Research Project (Survey, Interviews and Focus Groups)

\footnotetext{
${ }^{8}$ However, both of these organisations are currently being disbanded by the Newman LNP government, and will be subsumed into Brisbane City Council and the Department of State Development.

${ }^{9}$ Of these, four were consultancy firms, and one was a large development firm
} 
Property development was the sole business of just over half $(55 \%)$ of respondents, with other sectors including construction, mining, environmental auditing, engineering, general consulting, architecture, infrastructure, town planning, and organising major events. Of the developers themselves, two of the large developers ( $>\$ 20$ million turnover) also operated in construction, mining and retail and commercial investment. Of the medium sized developers ( $\$ 10$ to $\$ 20$ million turnover), two also operated in civil construction, consulting, and project and property management. Of the smaller developers ( $<\$ 10$ million turnover), three also operated in retail and property ownership and investment, and commercial leasing.

In the interviews and focus groups, larger scale developers generally had more adaptive capacity, not only because of greater financial and staffing resources, but because they tended to engage specialist consultants. They also tended to work over longer time frames, were often publicly listed, and were very concerned about their reputation and brand. The majority of larger developers interviewed stressed the issues of leaving a legacy, and the desire to create long term communities.

Developers had varying levels of sophistication; i.e. three consultants expressed the view that many developers were unsophisticated, with a short term outlook. This was, to some extent related to the size of the developer. Some smaller developers thought that adapting to climate change should only be the responsibility of larger developers; as they only operated over short time frames. As a consultant stated: "Most developers only ask three questions: is it possible (i.e. can they get an approval), how much is it going to cost, and how long will it take?" (CS6, Consultant).

On the other hand, some smaller developers were seen as having vision and passion; were self-educated, and were driven to produce quality products. These strongly motivated developers often used specialist consultants to provide additional advice and technical capability. However, these developers were generally an exception in the industry, as the majority of smaller developers had neither the capacity nor the financial resources to conduct extra studies.

Nevertheless, most developers who had survived the GFC were considered to be relatively professional, as "all the cowboys went bankrupt during the GFC" (SD1, Small Developer).

This accords with research showing that a predictable, calculated return on a given investment is not always the aim of developers, and are motivated instead by status or achieving high development standards (Coiacetto, 2001). These developers may have independent sources of finance and a given return may be secondary to the motive of 'making a statement'. On one hand, such developers might be less risk averse and more innovative (potentially useful in adapting to climate change) and may set high environmental standards. On the downside, they may also be attracted to high risk areas for physical climate impacts-i.e. waterfronts, rivers and beachfronts.

However, this study found that most developers were motivated by financial returns, and the desire to reduce financial risk. Some developers were seen as "just in it to make as much money as quickly as possible" (SC2, Sustainability Consultant). Further, some interviewees felt that this was, "aided and abetted by the banking industry, which are even more conservative" (LG1, Local Government). Especially for smaller developers, lending practices tended to drive certain behaviours, such as reluctance to build innovative products.

In general, lenders were extremely risk averse. They based their lending decisions on the proposed development product, and were more willing to lend to developers who had successfully completed similar products in the same geographical area. This tended to discourage most developers from innovation. To a major extent, the GFC had tempered most willingness to be innovative and to take risks. Most developers 
were highly risk averse and conservative due to the combination of the GFC, lending difficulties and regulation issues.

Moreover, many respondents expressed the opinion that smaller developers could no longer compete with larger developers, particularly in difficult economic times, and that the development industry would end up with the big developers in a similar position to the major Australian grocery chains, and driving out smaller competitors.

The majority of residential developers wanted to complete and sell their development products as quickly as possible. This was directly related to the constraints imposed by lenders, who generally only allowed 18 months to a maximum of two years to repay loans. This was true of all firm sizes; however, due to the scale of the development products they produced, larger firms generally staged their developments over a longer period, and were less affected by short lending cycles. In some cases, firms held back their higher value land because of a perceived lack of market demand in the current economic climate. In general, for the residential market, once all development products were sold and the bank paid back, most developers wanted to move on, and concentrate on their next project.

Although a longer term development project can increase exposure of a developer to the direct risks of climate change, it is still relatively short term, and the commitment of an individual developer to a site might be less because such projects often change hands. This short-termism has implications for adaptation for climate change. The products of development are enduring, and may be exposed to climate risks long after the developer has left the site; and become the responsibility and risk of others. This short term commitment versus long-term consequences is fundamental to the issue of sustainability in property development.

This was backed up by the findings of the study. Virtually all interviewees and focus group participants ( 21 out of 25 , or $84 \%$ ) commented on the temporal nature of the industry and how this influenced development. This study found that development projects ranged from very short term (i.e. a year to plan, construct and sell), to long term developments, such as master planned communities, which can take 25 years or longer to fully complete.

Many developers expressed a desire to, "get in and get out as quickly as possible" (CS1, Consultant). However, the timely completion of developments was often difficult to achieve, particularly for smaller developers. These developers were more at risk from the timing costs of changes to the planning and regulation system or even from their own attempts to introduce innovative design elements. For example, approvals for one development were held up for years by LGA planners, unsure how to deal with a proposed on-site stormwater treatment and reuse plant. Therefore, to alleviate some risk, especially from time delays, developers commonly put aside contingency funds for unforseen issues.

However, some developers were less constrained by the exigencies of bank finance. One developer interviewed had a high net worth business partner and another lead consultant was working for a similar interstate investor. Organisations with access to other sources of finance can afford to take a more relaxed approach to gaining approval or seeking changes to development consent conditions.

In addition, many larger developers practise land banking; buying and holding large sites for future development. These developers can also take more planning risk, by purchasing unzoned land, with the intention of future rezoning. One smaller firm was also developing a site that had been owned by their family for many years. This is relevant to the adaptive capacity of developers, as when a potential developer already owns land, they can afford to take a longer term approach to dealing with planning and regulation (J. Ratcliffe, Stubbs, M. and Keeping, M., 2009). 
The majority of smaller developers saw the practise of land banking as detrimental, as it prevented them from purchasing large or desirable sites. Moreover, many of these firms did not have the cash flow to hold on to large tracts of land. On the other hand, some developers got around this by not directly purchasing the land, but by entering into agreements with the landowners, paying them in instalments with sales of development products.

Nonetheless, even developers with fully owned land could incur significant extra expense from delays to approvals; as government charges, such as land taxes and rates, added significantly to holding costs.

\begin{abstract}
As a developer stated: "People don't understand that about development, you have to buy on a big margin because everything is put in your way to erode that margin, whether it is planning approvals, the market, a whole host of reasons, and time is your biggest killer"; and "developers are not a charity; they are there to make a profit. It is scary how much taxes and charges are on land" (SD1, Small Developer).
\end{abstract}

How development fits into a corporation's other activities can affect how it deals with different risks (including climate risks). For example, a multi-portfolio firm may engage in a range of different risk-level activities, such as undertaking a high risk development with a potentially high return (i.e. a marina development) together with a range of other lower risk activities. This strategy will be different to a developer who carries out development as its sole activity. Not all firms will respond to climate change risks in the same way.

A number of interviewees agreed with this, and felt that diversification was key to their long term success, giving the ability to ride out the various economic cycles. In general, developers felt that concentrating on only one type of development was too risky, and most developers who had done so in the past "went broke during the GFC" (CS3, Consultant). For example, one developer based his business model on that of larger firms; his development portfolio included a mixture of commercial, industrial, retail, apartments and residential lots.

On the other hand, some participants felt that gearing, not diversification was the most important success factor. Most developers who had gone bankrupt during the GFC were highly geared, and couldn't service bank loans in a falling market. Yet others said that successful developers created a niche, and maintained good relationships with the LGAs in which they developed.

\title{
4.2.2 Development process
}

When examining developers' capacity to adapt to climate change, it is important to consider the property development process. This is because different climate change related adaptation requirements may be present at different stages of the development process, which could result in financial or other implications, depending on the stage of the project.

For example, a developer may be required to use specific building materials and/or change building designs to comply with regulations in order to receive a building permit. If these are accounted for in the early stages, a developer may realise that the costs associated with the development are higher than initially calculated. This, in turn, could impact on both the capacity of the developer to borrow money, and the feasibility of the development.

Using a development process framework to examine adaptation issues can minimise the potential to overlook important challenges or opportunities arising from climate change. By using such a framework, it is easier to explore the potential challenges and opportunities comprehensively and methodically. 
However, as with development firms, the development process is diverse and multifaceted, and no generic model can be applied to any given development (Gore \& Nicholson, 1991). The development process is generally categorised by any one, or a combination of approaches: sequential or descriptive; behavioural or decision-making (emphasising the roles of different actors); production based (a macroeconomic perspective); or structures of provision (differentiation according to institutional, financial, and legislative frameworks) (Gore and Nicholson, 1991).

This study focussed on the sequential or descriptive approach, e.g. (Birrell \& Gao, 1997; Miles, Berens, \& EWeiss, 2000; J. Ratcliffe, Stubbs, \& Keeping, 2009; Syms, 2002). However, it must be noted that the steps in each approach do not vary significantly (Miles et al., 2000, p. 5), keeping in mind that (a) no two developments are the same and (b) the processes are often complex and (c) the sequence of events are rarely linear. In this approach, the phases of the property development process include: market research; site selection and investigation; feasibility studies; financial analysis; planning and development applications; purchase; design; construction; marketing; and sales and/or letting (Figure 10) (Birrell \& Gao, 1997).

\begin{tabular}{|c|c|c|}
\hline \multicolumn{3}{|c|}{ Site Selection } \\
\hline \multicolumn{2}{|r|}{ site investigation } & feasibility studies \\
\hline \multicolumn{3}{|c|}{ Project Planning } \\
\hline $\begin{array}{c}\text { planning \& development } \\
\text { applications }\end{array}$ & $\begin{array}{l}\text { community consultation } \\
\text { (if required) }\end{array}$ & $\begin{array}{l}\text { Itation operational works } \\
\text { applications }\end{array}$ \\
\hline \multicolumn{3}{|c|}{ Financing } \\
\hline financial modelling & $\begin{array}{c}\text { financing (research \& } \\
\text { application) }\end{array}$ & site purchase \\
\hline \multicolumn{3}{|c|}{ Design and Construction } \\
\hline $\begin{array}{c}\text { design (architecture, drafting, } \\
\text { landscaping) }\end{array}$ & $\begin{array}{c}\text { tendering/contracting } \\
\text { (consultants, construction etc) }\end{array}$ & $\begin{array}{l}\text { earthworks, construction and } \\
\text { landscaping }\end{array}$ \\
\hline \multicolumn{3}{|c|}{ Marketing and Sales } \\
\hline marketing and promotion & $\begin{array}{c}\text { creation of new property } \\
\text { titles }\end{array}$ & letting and/or sales \\
\hline
\end{tabular}

Figure 9. Stages in the property development process (Adapted from Birrell and Gao, 1997) 
Of note, few development projects contain a standard sequence of events, and nor are they undertaken in a linear fashion-much variability exists in the makeup and chronology of the phases (Birrell \& Gao, 1997). For instance, marketing and promotions and even sales may commence before any physical development of the site, especially where off-the-plan pre-sales are involved (Healey, 1991, p.224). This is almost always the case in the post GFC environment, with lenders requiring $75 \%$ or more pre-sales before they will consider funding a development. Further, a residential subdivision might not include any building at all, while a new building development may not include any subdivision at all.

\section{Development Process}

This study found that the development process was highly diverse, and very risky. Many interviewees stated that development was all about risk management. In the words of one developer, "what we do is try and remove as much risk as possible and come up with a good product" (MD3, Medium Developer). Of note, despite the impacts of the GFC, many smaller developers were still highly leveraged; for example, some had mortgaged their own homes against their developments.

The development process was likened to the manufacturing process, only less efficient, and with slow technical innovation. A common theme was the lack of efficiency in the development process. It was felt that improving efficiency would significantly help to reduce costs.

\section{Selection of Site and Location}

With regard to geographical area, the research specifically targeted developers and consultants who operated in SEQ. However, many also developed in other areas, especially resource areas such as Mackay and Gladstone. The majority of survey respondents (75\%) operated in SEQ; but others worked elsewhere in Australia and some consultants worked internationally. Within SEQ, respondents operated in most regions, with a slight preference for the outer suburbs (from $10-25 \mathrm{~km}$ from the Brisbane CBD) and nearby urban areas such as Caboolture and the Sunshine and Gold Coast (61\%), with slightly fewer in the CBD (Table 4). Of the interviewees, two Brisbane based developers also operated in northern New South Wales.

\section{Table 4 Proportion of developers working in various regions of South East Queensland (Survey Respondents only).}

\begin{tabular}{|l|l|}
\hline Area of Development & Percentage $^{\mathbf{1 0}}$ \\
\hline Brisbane CBD & $38.5 \%$ \\
\hline Inner Urban (<5km from CBD) & $57.7 \%$ \\
\hline Inner Suburban (5 - 10km from CBD) & $50.0 \%$ \\
\hline Outer Suburban (10 - 25km from CBD) & $65.4 \%$ \\
\hline Regional Commuter (25 - 100km from CBD; i.e. North Lakes, & $50.0 \%$ \\
\hline Regional Urban (Gold Coast, Sunshine Coast, Ipswich) & $61.5 \%$ \\
\hline
\end{tabular}

The interviewees and focus group participants felt that location was critical. In many cases, developers chose sites based on the economy of the region, and the risk profile of the site itself. Areas with wide, diverse economies were preferred to those with narrow economies. For example, Townsville in North Queensland, was considered to be a good market, due to having a more diverse economy than single economy towns,

\footnotetext{
${ }^{10}$ Note, respondents could select more than one area, so percentages add up to more than $100 \%$.
} 
such as Dysart (mining) or Airlie Beach (tourism). These areas were considered as extremely risky, because they had single sources of income, and were more vulnerable to economic cycles and downturns.

In SEQ, developers tended to concentrate in certain geographic areas because of local knowledge and increased likelihood of bank finance. Many developers preferred outer suburban or greenfield areas, from $10-25 \mathrm{~km}$ from the Brisbane CBD. This was largely due to the greater availability and lower cost of large land parcels. For example, some smaller firms stated an unwillingness to develop in the CBD or inner urban areas, due to high land prices and oversupply of product. Nonetheless, some developers, mostly catering to higher income clients, specialised in waterfront or oceanfront areas.

Site selection was difficult for smaller developers, because of the practice of land banking by larger developers. Some smaller developers felt that the larger developers already owned the best sites, and if a potential developer hadn't entered the market 10 years ago, they were already at a major disadvantage. For this reason, some developers bought land outside SEQ, as it was cheaper.

Prior to purchasing a site, most developers conducted extensive due diligence research. Developers investigated sites themselves, and used consultants, such as surveyors and town planners. They also used LGA and State Government Geographic Information Systems (GIS) and satellite imagery (such as NearMap ${ }^{11}$ ) to investigate sites for problems, such as flooding. One developer pulled out of a site purchase because it was supposedly flood free, but NearMap showed it had been inundated in the 2011 Brisbane floods. The physical attributes of a site were extremely important.

Site selection was also opportunistic as, in many cases, potential development sites were not for sale. If a potential development site was less than optimum (i.e. was lowlying and subject to flooding); extensive cost benefit analyses were conducted on the expense to fill the site prior to purchase. Most developers and consultants felt that site issues were manageable with engineering solutions, as long as they were cost effective. However, it was also noted that filling sites or major earthworks, could impact neighbouring properties, resulting in possible litigation.

Another potential issue with site selection was contaminated land, which can add significantly to costs. It is possible to search State Government databases for contaminated land, but not all sites are recorded; and contamination can remain unknown until after purchase, when preliminary earthworks are undertaken. One financier spoke of a site costing $\$ 1$ million and after the discovery of contamination; the site cost over $\$ 3$ million to remediate, thus negating any profit from the development.

Climate change was considered just one risk amongst many when selecting development sites. For example, developers looked for the best land, which was generally not flood prone-the market tended to pay more for elevated land with views. Moreover, developing flood prone land was considered extremely risky, because locals would be aware of this issue, and this would restrict the market to external or interstate buyers. Further, the banks would likely not fund developments on flood prone land.

One issue with site selection was not whether the site was subject to climate events, but whether access routes were at risk. A consultant spoke about a LGA development refusal which was overturned by the Planning and Environment Court. Although the site was flood prone, the court felt the developers had provided sufficient contingencies for the residents to evacuate in an emergency.

\footnotetext{
${ }^{11}$ A firm specialising in high resolution satellite and aerial images; http://www.nearmap.com/
} 


\section{Project planning}

Project planning includes market research and analysis, feasibility, due diligence and cost benefit studies, and planning and operational works applications. In most cases, financiers expected this to be completed prior to granting any loans. Financiers often requested full feasibility studies, including comprehensive valuation and quantity surveyor reports, detailed lot layout plans and location of parks. As part of project planning and feasibility studies, developers frequently attended pre-lodgement meetings with LGA and State planners and engineers.

These were considered onerous requirements, as the costs of hiring specialist consultants to conduct studies could run into hundreds of thousands of dollars even before the developer was granted finance. However, other developers said that they would conduct as many studies as necessary (and as many as they could afford) to reduce as much risk as possible.

The stages of the development process generally depended on the size of the developer. Smaller developers tended to have a few staff, each specialising in certain aspects of development. For example, one small developer had a dedicated staff member for each construction, planning, accounting and design role. However, if other skillsets were required, the tasks were outsourced to consultants.

Project planning was intrinsically related to the location and physical characteristics of the development site. For example, a sustainability consultant stated:
"We look at location and design, the stakeholders that are moving in, the destruction of landscape. We look at each of the different elements and each of the elements, such as around sea level rise, temperature, rainfall, wind and the like would have an impact on those development elements, and we provide an overall rating and recommendations around adaptation measures" (SC1, Sustainability Consultant).

Generally, most planning and development applications were conducted by consultants, and not by developers. Even the larger developers used consultants to navigate the maze of legislation. However, one developer noted that consultants sometimes missed important information. Two sites were being investigated for a feasibility study, and the developer dropped one because he knew a forthcoming State Planning Policy would impact on the site.

Although community consultation is a key aspect of the development planning process, particularly for larger developments and those requiring Environmental Impact Assessment; few participants mentioned this stage. However, it was implicit in discussions about community building, and leaving a legacy (see Chapter 7).

Of note, some large developers and consultants reduced planning risk by employing lobbyists, or used the Property Council of Australia (PCA) to directly lobby government, to change planning laws to permit development.

The regulatory aspect of project planning is discussed in further detail in Chapter 5 , Governance and Regulation.

\section{Design and construction}

Larger developers generally designed their own products using in-house architects and drafters. Many of the large project builders produced variants of only a few plans, using what a respondent termed "matchstick construction" (AR1, Architect). These detached dwellings were considered efficient, and cost effective, because they used standard sized fittings, and many of the components were made up in kit form in factories. They were also simple to erect, and could be managed by the builder's apprentice on site. 
However, the majority of developers used external consultants and subcontractors for design and construction. Some developers and consultants felt that subcontractors had too much influence on the final product. This could have significant implications for adaptation to climate change. In the words of a consultant employed by a large firm:

\begin{abstract}
"If builders and contractors can make a saving, they will cut any adaptation initiative that they can, if they see it as a cost or if it hasn't been securely locked into a contract or consent approval, it will definitely be left off. It is very important to consider adaptation right at the early stages so it is just embedded as part of the concept plan or concept design or the master plan for a project. So that it is right there for the start. If you try and bolt it on at the end, it is not integral as part of the project. That will then be identified by the contractor or the constructor as a cost saving. And they will cut corners" (SC1, Sustainability Consultant)
\end{abstract}

Large development firms also produced a wider diversity of product, from shopping centres, to luxury riverfront apartments, to very large master-planned estates. The majority of these firms used in-house staff for everything from initial planning through to construction. Others tendered out various aspects of the development process, such as infrastructure or landscaping, to consultancies. However, sourcing contractors in certain areas was quite difficult; a medium sized developer said that North and Central Queensland developers had a problem keeping contractors, as they were frequently "poached" by the resources sector.

With regard to climate change, developers didn't want to build anything that would leave them liable for a higher level of risk than their competitors because they felt this would over-service the development, and impact on final profits. For example, one developer stated that he could build Category 5 Cyclone rated houses on the Gold Coast, but as none of his competitors were doing so, he would soon go broke.

"At the end of the day, they <developers> are builders. They build a product and sell it and they need to turn it over as quickly as possible. that doesn't mean some of them don't have a commitment to do the best job they can with built form, materials, orientation, all those sorts of things but they are not going to spend one cent that's not going to return to them" (CS1, Consultant).

\title{
Financing
}

Many developers face major uncertainty whether a project can obtain funding, particularly from conventional sources, such as banks. A developer has to invest considerable resources and time before an institution will even consider funding a project. This may include negotiating a land purchase deal, obtaining development approval and completing a comprehensive market study. Nearly all participants in this study reported increasingly onerous and costly conditions imposed by the banks, as well as lower Loan to Value Ratios (LVR) and increased security requirements (J. Ratcliffe, Stubbs, M. and Keeping, M., 2009).

There also risks concerning the loan conditions, duration, repayments and interest rates. All but the largest developers reported increased difficulty in obtaining finance since the GFC. The 'Big Four' banks, ANZ, Westpac, NAB and Commonwealth, were the commonest sources of funding; as many medium and smaller banks were, "burnt during the GFC" and no longer focus on development funding (FI2, Financier).

This difficulty in obtaining finance, together with the implications of the GFC, can influence a developers' ability to adapt to climate change. Bankers were unwilling to fund innovative development products that were untested in the current market, preferring to fund products similar to those built and sold in the past. 
The extreme risk aversion of financiers largely did not support developers wishing to experiment with new and more sustainable products.

Given the integral nature of financing to the development process and adaptation to climate change, it is discussed in greater detail in Chapter 6, Finance and Market.

\section{Marketing and sales}

Another serious risk faced by developers is selling or letting development products; as it can be challenging to anticipate the state of supply and demand at the completion of the project, because this rapidly changes in response to external factors, such as economic cycles and interest rate changes. Residential markets are also highly segmented and each submarket, such as first home-buyers or investors, require different competitive strategies (Coiacetto, 2007).

This study found that virtually all developers and consultants considered that one of the major sources of risk in the development process was marketing and selling the products of development. Some developers conducted their own marketing, but many used specialised consultants to do market research, and run the marketing campaign.

Bank's requirements exacerbated marketing issues. Many developers (and banks) reported that they were unlikely to obtain funding unless a significant percentage of pre-sales was recorded. In some cases, banks required a minimum of $75 \%$ (or even $100 \%$ ) of pre-sales before they would consider funding a development. Therefore, it was crucial to successfully market and sell developments as rapidly as possible.

Further, many respondents stated that they would not undertake any sustainability or climate adaptation measures unless there was a marketing advantage to be gained by doing so. Despite this, both a financier and a State Government representative emphasised that the better developers still produced high quality, sustainable developments, regardless of marketing advantage. In addition, a medium sized developer, noted for innovative and high quality products, expressed excitement over the possibility of building houses that were totally "off the grid". However, the developer stressed the real issue was getting the market excited about it, and at present, buyers were more concerned about price.

In general, the current economic situation and difficulties in obtaining funding made marketing sustainability extremely difficult, particularly if upfront costs were higher. Prior to the GFC, more developers had been willing to try innovative or "green" designs to gain a competitive market edge. However, since the GFC, very few were willing to take this risk. A small developer from Brisbane summed this up as follows: "Climate change is how you feel. Some purchasers are concerned about it and some are not, some are more concerned about having a greener house and some are not." (SD2, Small Developer).

Marketing and the provision of "green" developments is further discussed in Chapter Six, Finance and Market.

\subsubsection{Development type}

For the survey respondents, most projects were relatively large residential developments; in excess of 50 lots or dwellings. Few developers focussed on smaller, "Mom and Dad" type projects, such as a "six-pack" of units, or on large, mixed use development. The commonest type of development was reconfiguration of lots-i.e. land subdivision, cutting up a large piece of land into smaller lots, constructing the infrastructure, and then selling the smaller lots (Figure 11). Developers also worked in building construction, but tended to construct attached buildings (multi-storey residential and townhouses) rather than detached dwellings. 
The area of development was correlated with the type of product and land prices; with greater numbers of land subdivision and detached dwellings in the outer suburbs and regional areas, in comparison to the inner city. Fewer numbers of developers worked in temporary dwelling construction, such as mining camps, renovations of existing buildings; and redevelopment (typically from industrial or commercial to residential).

Developers also specialised in specific market sectors, with the major types being aged care, affordable housing, and "family" type housing. Other specialty areas of development included first time homeowners, social housing, student housing, tourism, high end residential, and specific locations, such as waterfront or rural residential.

Developers said that some housing types were more efficient and cheaper to build. For example, detached project houses were considered one of the most efficient construction types because, "everything is standardised and out of the box" (LG1, Local Government). Other housing types such as three storey walk-ups were seen as more efficient and cheaper to build, but there was no demand for these.

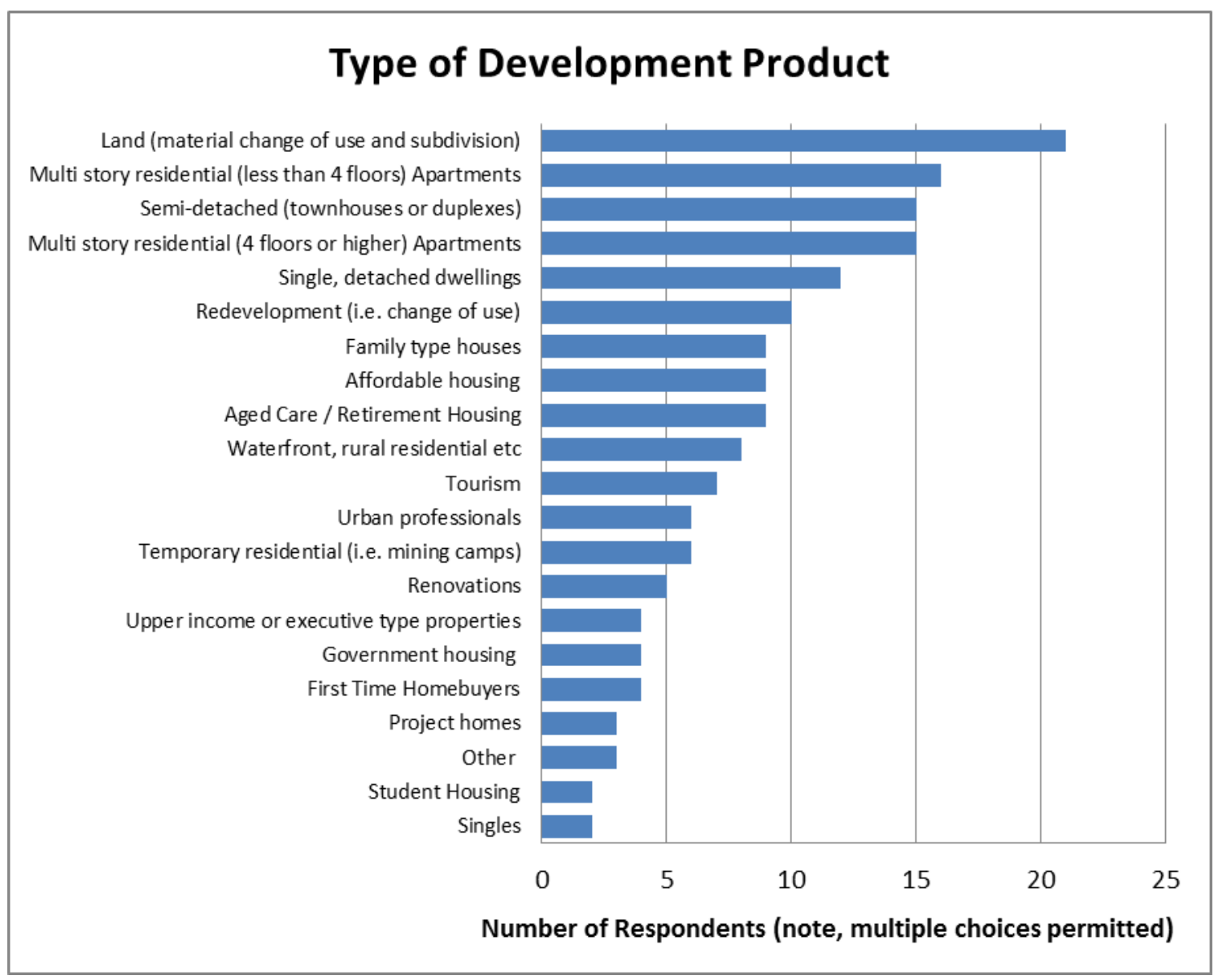

Figure 10 Type of Development Product (Survey Respondents)

The majority of developers subdivided land, and sold vacant lots. Lot sizes have reduced significantly in recent years (Hall, 2009) and although governments consider this a result of densification policies, the developers disagreed. An employee of a large development firm said that smaller lots had nothing to do with policy, but everything to do with government charges and the selling price of their products. Developers wanted to keep lot frontages as narrow as possible, to deliver more affordable land, and appeal to a wider market. 
A major issue was delivering affordable housing. Developers felt that this problem was exacerbated by the range of taxes and charges imposed on land, from all levels of government. In some cases, these charges could add up to $40 \%$ of the price of a block. One developer stated, "we are being taxed out of being affordable and regulated out of being affordable and that is the problem" (SD2, Small Developer). Nevertheless, the provision of affordable housing was considered a potential driver for climate change adaptation. Some affordable housing designs incorporated rainwater tanks and energy efficiency measures, and were attractive to price-conscious buyers who also wanted to reduce long term operating costs.

Developers correctly viewed their market as highly diverse, and sought to produce diverse products to target different market sectors and environments. However, they felt that the inflexibility of the built and the urban form locked them into a certain type of product. Further, they felt that the emphasis of the South East Queensland Regional Plan on encouraging compact urban form precluded some innovative products. Finally, the planning system was seen as confusing housing need with housing choice; for example, some wanted small, compact, attached houses in the CBD, but many others still wanted space and a backyard, especially if they could afford it.

\subsubsection{Consultants and Other Stakeholders}

A common operational strategy is to manage risk by operating in a limited area, and to rely on local information and contacts (Logan, 1993). For example, development funding is often easier to obtain for developers who have, with past projects, shown a good knowledge of the local area and the sub-markets within. However, when developers operate in markets and regions other than where they are based, the most common strategy is to use the services of local consultants.

Consultants are commonly used by developers to navigate the complex regulatory environment. However, the costs of hiring consultants can be onerous for smaller operators, and such costs may represent barriers to entry and participation in the industry and affect competition. This, in turn, can have flow-on effects, such as the capacity of industry to deliver innovative climate mitigation or adaptive designs (Coiacetto, 2009). Thus, smaller developers often have to rely on their own ability to acquire knowledge of the complex, and sometimes rapidly changing regulatory environment. This may leave them liable to insurance or legal claims if they omit to keep up to date with the regulation.

A developer or peak consultant coordinating sub-consultants has more control of what goes into the final product, but also increased risk because, "the financial trail goes through you" (AR1, Architect). Such developers are reliant on the expertise of the subconsultants and if these made recommendations (for example, regarding an energy saving innovation) and the predicted results do not eventuate, the developer may be liable for any costs or legal action. This issue can sometimes lead to perverse incentives, such as cost and time "rewards" for being reactive and conservative.

In this study, a range of consultants were interviewed and surveyed. Like developers, consultants differed widely, with firms ranging from large multi-national ASX-listed companies, to single person operations. Some consultancies operated more as head contractors, coordinating a range of sub-contractors and consultants. Consultants also tended to specialise in specific fields, including planning and climate adaptation.

Similarly to developers, consultancies had been hit hard by the GFC, with two reporting losing $50 \%$ or more of their staff during the GFC.

Most larger (and a few smaller) development firms outsourced all their expertise to consultants. The most common consultancy services offered were conducting and submitting planning and development applications to government, and engineering services. 
Many larger consultancies also used sub-consultants to conduct further research. Consultants were also limited by data availability; some expressed frustration with State and Local Government data provision; much government data was expensive, inadequate, poorly communicated, and difficult to obtain. Another issue with consultants was the reliance on grey literature, and previous consultancy reports.
"Engineers by and large are not necessarily doing the research, or have the time to do the research which is necessary, so we rely on other bodies to present us with the information we should use, so that probably means engineers are quite reactive as well in terms of dealing with these types of issues" (CS8, Consultant).

Consultants had varying degrees of influence over the development process, depending on whether they were considered a part of the development team. Sustainability consultants said their worth was judged on how much money they could save the company-unlike engineers or planners, who were seen as integral to the development process. Not all developers viewed consultants favourably: "Consultants tell developers what they are legally obliged to do, and they don't like it" (CS3, Consultant).

Most consultants and professionals, such as architects and engineers, are bound by codes of conduct, which require them to be environmentally responsible. One engineer commented that previously the firm only had to be concerned about engineering constraints, but now they also had to be "across the legislation" (CS8, Consultant). This was thought to have substantially changed the field for engineers and developers.

Finally, it was considered that professionals such as consultants, could instigate change to a certain extent, but they were often limited by the development or construction manager. A few respondents, including a banker, stated that builders deliberately made money out of regulation changes, by issuing variations.

\subsection{Conclusions}

In accordance with previous research, the property development industry, together with allied professionals, such as consultants, was highly heterogeneous. A wide range of firms practised property development activity; and projects ranged from large, multistage, master planned developments to small townhouse complexes. Common themes arising from the research included: the diversity of developers and their product; the time and scale of development; the difficulties in obtaining finance; and the overarching importance of the market. The importance of consultants to the development process was also highlighted.

These all have implications for adaptation to climate change, which presents a variety of risks to developers; which are more or less relevant to different developers. Moreover, how developers evaluate and deal with climate risks will also be different. For example, some firms may be willing to accept a bigger climate change risk (i.e. developing in popular coastal areas) instead of taking a lesser risk in another area, such as market risk (i.e. developing in an inland area).

Further, all developers' exposure to physical climate risk is limited by their relatively short commitment to a site or project compared to the enduring nature of their development products. Even the longest term development is relatively short term in comparison to the built product, which may endure for a century or longer. Arguably, developers may have a long-term social and environmental responsibility to mitigate and adapt to the risk of climate change hazards for the eventual users of their products. 
Development projects also require a considerable investment of financial resources, so gaining access to funding is critical for developers, and for development to occur. However, because of the risk involved in development, gaining access to finance is traditionally problematic for developers. Moreover, the constraints imposed by financiers also have direct implications for adaptation to climate change.

In the post GFC environment, financiers have become increasingly risk-averse.

Developers noted that banks were very unwilling to fund new or innovative products, which were untested in the market. Further, the time frames required by bankers to repay loans were typically under two years, and this further exacerbated the short term focus of the development industry.

Marketing and selling was absolutely fundamental to development, as most developers wish to profitably sell or lease their development products. It was also fundamental to obtaining finance, as many banks would not lend money unless $75 \%$ or more of the development had been pre-sold. Few developers reported any demand for "green" products by the residential market. The only exception was the retirement housing sector, but this was based on a desire to save money rather than for sustainability.

Finally, consultants were commonly used throughout the development industry, in a range of roles, from specialists, to development coordinators. Consultants were regarded as an important source of expertise, and often used to provide extra "checks and balances" to reduce risk to developers. 


\section{GOVERNANCE AND REGULATION}

\subsection{Introduction}

The previous chapter showed how property development firms differed widely in both their organisational structure, and their operational strategies; and how these determined the operating ability, and the extent of their capacity to adapt to climate change. However, equally important to adaptation is the regulatory settings under which private property developers operate.

Property developers operate within a diverse environment of complex legislation, regulation and economic forces. Climate change adds to this complexity, with changes to regulatory mechanisms aimed at supporting climate change adaptation and mitigation efforts.

Regulatory changes are occurring across all levels of government in Australia, and include the Carbon Tax legislation (National) and the (recently suspended) Coastal Plan (Queensland). Regulatory changes may also come from within the industry or from associated institutions, such as financiers and insurance companies.

The implications of changes to the policy and regulatory framework for developers can be significant, ranging from an increase in the cost of development to permit refusal and legal challenges (Briggs et al., 2010; Taylor, Harman, Heyenga, \& McAllister, 2012; Urban Development Institute of Australia (UDIA) South Australia, 2011, p. 3).

As a business, the sector has to operate under the relevant legislation, policy and regulation pertaining to the type of development it practises. Some legislation, for example, the Federal Carbon Tax, does not directly impact many smaller developers, but may indirectly impact them due to supplier price increases. However, most developers are directly impacted by the Building Code of Australia (BCA), State legislation such as the Sustainable Planning Act, and LGA planning schemes. In addition, many development firms adhere to a wide range of voluntary standards and certification measures.

Planning and regulatory risks are often approached differently by different developers. Most developers will adhere to the relevant planning constraints imposed by the Local or State Government agency, or the BCA. For example, developers may only seek appropriately zoned land; i.e. a site in the SEQ Regional Plan's Urban Footprint, above the Q100 flood level ${ }^{12}$. However, other firms may choose to risk a long term approach to seeking land for their development purposes; such as purchasing unzoned land, or filling low-lying flood-prone areas.

Different developers also respond to regulation in different ways. Planning and regulatory risks are often approached differently by different developers. Developer strategies may place more emphasis on some risks than others (Coiacetto 2001; 2007). What strategy a firm takes, may depend on factors like political influence, the funding source and supply, and the firm's overall strategy.

Further, different types of firms have different capacity to absorb the costs of regulatory compliance. In general, the increasing complexity of regulation is likely more onerous for smaller operators. Such costs may represent barriers to entry and participation in the industry and thus affect competition in the industry, which in turn, can have flow-on effects, such as the capacity of industry to deliver on affordability (Coiacetto 2009).

\footnotetext{
${ }^{12}$ The height of a flood level that has the probability of occurring once in one hundred years. Used in Queensland LGA planning instruments.
} 
Further, smaller developers are less able to afford specialist consultants to navigate the planning maze, and often have to rely on their own ability to acquire knowledge of the complex, and sometimes rapidly changing regulatory environment. This in turn may leave them liable to insurance or legal claims, if they omit to keep up to date with the regulation, and the development is impacted by a climate hazard, such as flooding or bushfire.

This study found that the majority of developers and consultants were critical of government legislation. Most of the criticism was directed at Local and State Government regulations, but one larger developer commented on the onerous reporting requirements of Federal legislation. Very few respondents mentioned the Carbon Tax despite its prominence in the media.

Most developers cited the BCA as the most significant legislation for their businesses. Respondents generally supported the BCA, and considered that these were sufficiently flexible and responsive to adapt to future climate change. In addition, they felt that if they adhered to the provisions of BCA and other regulations, they would be protected from any litigation regarding climate events.

\subsection{Regulation, Legislation and Planning}

\subsubsection{Federal Policy}

The Clean Energy Act (aka the Carbon Tax) sets up a carbon pricing mechanism in an attempt to reduce carbon pollution in Australia. This tax affects approximately 500 businesses in Australia. In general, a threshold of 25,000 tonnes of $\mathrm{CO}^{2}$-e emission applies when determining if business is required to pay a carbon tax (Australian Government, 2011).

The Carbon Tax does not directly influence most developers, as they are not included on the list of affected businesses, but it may add to the costs of development, as supplier companies; such as concrete companies, are affected and will likely pass added costs on to their customers. However, this will likely differ between products and businesses (Deloitte Touche Tohmatsu, 2011, p. 6).

Prior to the implementation of the Carbon Tax, the industry's key concern was a possible sharp decline in the demand for housing following the introduction of the tax (as happened after the implementation of the GST). Fears were for a loss of jobs in an industry which accounts for $7.3 \%$ of Australia's GDP. There were also concerns about the projected increase in the cost of housing $(\$ 2000-\$ 6000)$ and the subsequent erosion in housing affordability (Government News, 2011).

However, only a few survey respondents (5) felt the Carbon Tax was an issue for their business. These were all large developers or consultants, and no medium or smaller developers considered it would pose any serious problems to their businesses.

In the interviews and focus groups, few respondents (3) even mentioned the Carbon Tax, and none felt it posed a major risk to their businesses. One LGA employee said that it had had very little, if any, influence on local government, and none on developers. A major developer felt that the Carbon Tax had had some benefit, as it had made people stop and think about their energy use, and how they might save money in the long term by including energy efficient products in their home. The final respondent mentioned the Carbon Tax in the context of political uncertainty, amid a general criticism of the Federal Government. Nonetheless, a degree of uncertainty remains regarding the potential effects of the Carbon Tax on the property development industry, and the UDIA has called for industry-specific modelling to shed more light on potential future impacts (Government News, 2011). 
One component of the total Clean Energy Act package, the National Greenhouse and Energy Reporting (NGER) Scheme, was specifically alluded to by one large developer. The NGER scheme aims to track Australia's progress against the Kyoto Principles (Clean Energy Regulator, 2007). It is managed by the Clean Energy Regulator, and is relevant to larger developers, which are required by law to report their emissions and energy data (under the Australian National Registry of Emissions requirements).

The requirement to report emissions was thought to impose additional requirements on developers, significantly adding to staff workloads, as each government department required the same data, but in different formats. The developer considered that reporting the same data to suit varying government needs was time consuming and inefficient. They proposed that an alignment on data reporting across different levels of government and departments would significantly improve efficiency, compliance and analysis of emissions data.

\subsubsection{Building Code of Australia}

The most commonly cited Federal legislation was the Building Code of Australia (BCA), which forms part of the National Construction Code. The BCA sets out technical provisions for building, including resistance to fire and energy efficiency.

"The goal of the BCA is to enable the achievement of nationally consistent, minimum necessary standards of relevant, health, safety (including structural safety and safety from fire), amenity and sustainability objectives efficiently" (Australian Building Codes Board, 2011)

Most respondents alluded to the BCA (Figure 12), with $77 \%$ of survey respondents stating that changes to regulation, such as the BCA would have a significant or highly significant impact on their business. Developers and consultants also stated that they always followed the minimum requirements of the BCA and planning regulations for their projects. A financier said that the bank always assumed that consultants will inform them if the project has been signed off against the requirements of the BCA. Others felt that the BCA restricted what they could do; and as long as they followed these requirements, it protected them from any liability.

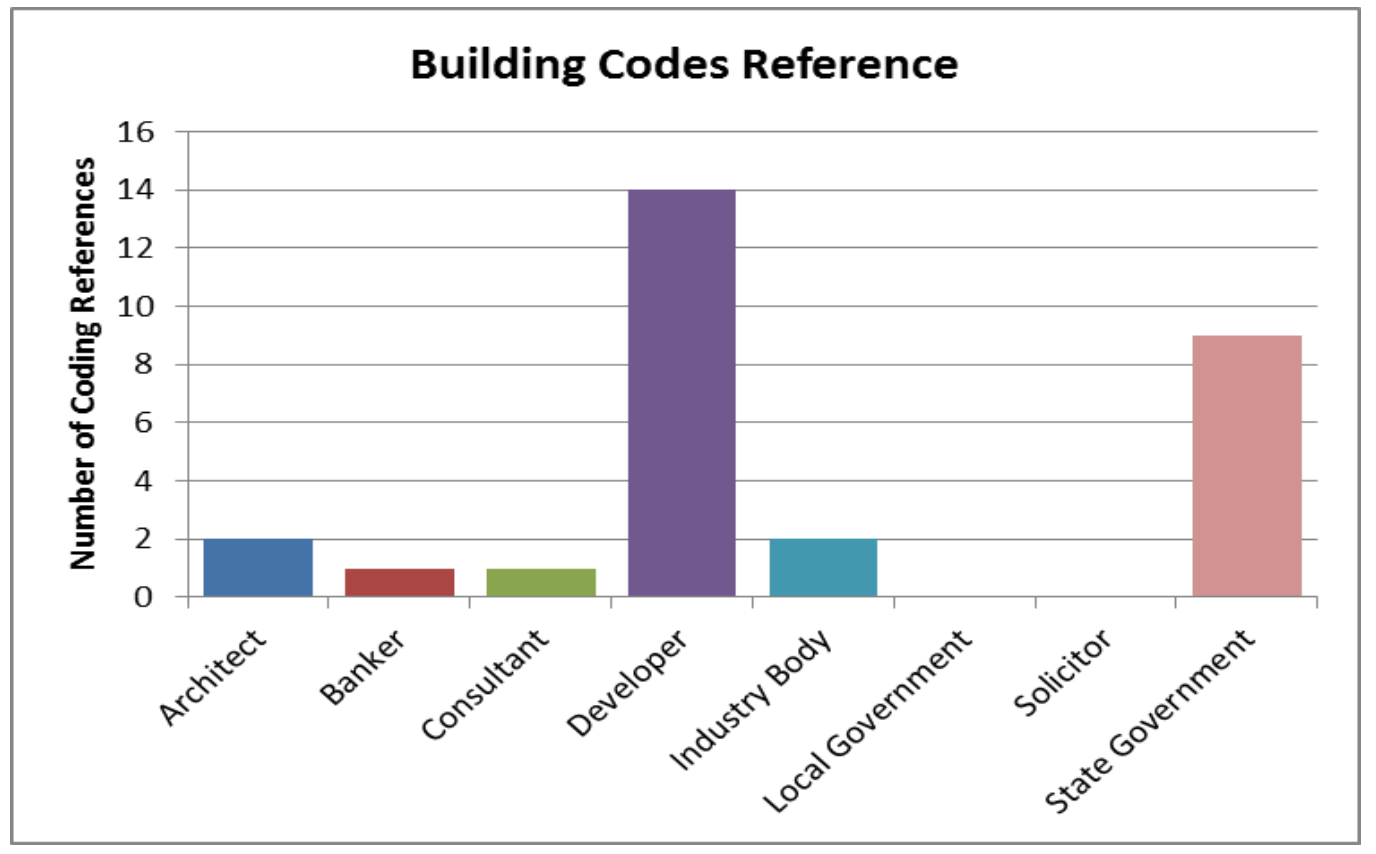

Figure 11 References to the Australian Building Code (and building codes in general) 
A State Government employee commented that LGAs try and cover everything in their planning schemes, sometimes resulting in duplication and inconsistency between the BCA and planning schemes. In addition, the BCA frequently did not interface with the planning scheme, because building certifiers looked at the BCA and not the planning scheme.

Some consider the BCA inadequate to protect buildings against the impacts of climate change; as it has no provisions to minimise the risk of inundation from sea-level rise, nor adequate provision for buildings outside the current cyclone limit (Department of Climate Change, 2009, p. 150). Arguably, there is an urgent need to review existing codes and modify them in the context of climate change. Delaying the process could potentially result in high economic losses due to the incompatibility of existing building stock to deal with extreme weather events and sea level rise (Department of Climate Change, 2011; Stewart \& Wang, 2011). According to DCC (2009) economic losses associated with extreme weather events could significantly be reduced by adopting a range of regulatory changes that target 'at risk' infrastructure and development.

However, the Australian Building Codes Board $(A B C B)$ is currently reviewing the $B C A$ in the context of climate change, by investigating design guidelines for building in flood affected areas and other areas prone to the impacts of extreme weather events (e.g. cyclones and bushfire). For example, the $A B C B$ has proposed to revise requirements for construction in cyclone affected regions, by shifting the boundary of Cyclone Region $C$ south to include the Sunshine coast, but not Brisbane (thus increase the design for wind force by approximately 50\%) (Australian Building Codes Board, 2012).

However, changes to the BCA are unlikely to provide an immediate protection from the impacts of climate change due to a considerable time lag between the implementation of new building codes, and their reflection in a majority of the building stock' (Department of Climate Change and Energy Efficiency (DCCEE), 2011, p. 17).

Nonetheless, most developers and consultants felt that adherence to the BCA was sufficient to cope with climate change. Generally, developers felt as long as they adhered to the minimum legal requirements in the BCA, they would not be liable for climate change impacts. They also felt that, as the BCA abided by Australian and International standards, they were adequate, and to some extent, best practice. LGAs also assumed that the BCA could effectively protect property at the dwelling or lot level.

Most developers and consultants thought the BCA was flexible enough to adapt to climate change, as these were constantly updated in response to climate events, such as cyclones. The attitude of most respondents echoed the following comments:

"Building codes are all based on probability; with changes in the future, the developer just moves where he builds on that probability curve" (CS8, Consultant); and

"Climate change is no bigger (risk) factor than anything else. The sustainability of houses has now been mandated in the building code" (SD2, Small Developer).

\subsubsection{State Policy}

Queensland State policy generally follows a hierarchical, cascading model. The overarching SEQ Regional Plan 2009-2031 (SEQRP) aims to provide integrated planning at a regional level. The main goals of the SEQRP are to manage growth, guide future land use patterns, and protect the environment and lifestyle (Office of Urban Management, 2009). 
Below the SEQRP, the Queensland Sustainable Planning Act 2009 (SPA) is the legislative framework for all planning and development undertaken in Queensland. The SPA aims to manage and regulate land use in order to achieve the most sustainable (environmental, social and economic) outcome; and seeks to direct development to the most suitable sites. It requires decision making processes to take into account the effect of development on climate change; and to lessen or avoid the adverse environmental effects of development (SPA 5(1) (a) (ii); SPA 5 (c) (i)).

In coastal areas, the overarching legislation (was) the Coastal Protection and Management Act 1995, which incorporated the Queensland Coastal Plan 2012 (Coastal Plan); the State Planning Policy 3/11: Coastal protection; the State Planning Policy for Coastal Management 2012; the Coastal Hazard Guidelines 2012 and the Adaptation Strategy Guideline. The Coastal Plan aimed to provide clear and consistent guidelines for coastal management and future urban development along Queensland's coastal areas (The State of Queensland, 2012b). LGAs that were impacted by the Coastal Plan were required to prepare an adaptation strategy for urban localities in the next 5 years, taking into consideration a range of options to mitigate coastal hazards.

The Local Government Association of Queensland objected to the Coastal Plan as it could severely affect new development in a difficult economic environment (LGAQ News Release online, 2012). Similarly, the UDIA and the PCA expressed discontent with the perceived haste in which the Coastal Plan was developed and introduced; and considered that it required extensive training and consultation to assist property owners, developers and LGAs to correctly interpret the policy (PCA 2012).

However, due to the recent change of State government, at the time of writing (October 2012) Queensland legislation and policy is in a state of flux. As of 8 October 2012, the State Planning Policy 3/11: Coastal Protection (SPP) has been suspended, and has been replaced with the Draft Coastal Protection State Planning Regulatory Provision; but the State Policy for Coastal Management is still in effect (Department of Environment and Heritage Protection, 2012).

Although part of the Coastal Plan has been suspended, it was still in effect during the research process. In addition, it was mentioned by the majority of respondents. However, as the Coastal Plan was the first piece of legislation in Queensland that attempted to respond to the risk of sea level rise, some findings are reported below.

The majority of survey respondents said they had an average to good knowledge of State legislation, including the Coastal Plan, the SPA, and the State Planning Policy 1/03; Mitigating the Adverse Impacts of Flood, Bushfire and Landslide. As was expected, consultants considered themselves to have the best knowledge (as these frequently deal with planning legislation). Small developers had the least knowledge.

Most interview and focus group respondents did not mention any specific legislation, with the exception of the Coastal Plan, and to a lesser extent, the Koala Plan and the Vegetation Management Act. A common criticism was that some legislation, such as the Koala Plan and the Coastal Plan, had been implemented too rapidly, were unnecessarily complex, and developers had been given insufficient forewarning of the legislative changes. It was also felt that the Coastal Plan was too broad, and could be interpreted in many different ways.

As one consultant stated, "the Coastal Plan is a very poor piece of legislation, has not been well drafted, <it> seems to be a knee jerk reaction to whatever the government thought they were trying to do. But it has created difficulties in the profession because it is such a difficult document to interpret" (CS8, Consultant). 
The major criticism of the Coastal Plan was the inclusion of sea level rise and climate change adaptation. It was felt that sea level rise should be separated from the Coastal Plan, as it required developers to ascertain the risk of sea level rise and climate events such as cyclones, and this had to be assessed by the LGA. Many respondents felt that neither developers nor LGAs knew how to interpret the Coastal Plan, and that if they did so incorrectly, they could be held liable in court. The Coastal Plan was also site specific, so it put the onus on the LGAs to assess risks for a site.

The Coastal Plan also referred to risks to future operations. For example, a LGA might permit a multi-use development in the coastal zone, with residential units situated on higher levels, and retail below. However, repeated flooding of the lower levels would impede the operation of that development; with a range of negative impacts, such as loss of trade, cost of replacement fixtures and fittings, and obstructed access by residents. For example, in the Brisbane floods of 2011, a commercial building that was flooded had lease applications for retail stores. One tried to pull out, but changed their mind after some negotiation.

In addition, it was felt that if the Coastal Plan were ever fully implemented, many existing developments would fail, because access routes were likely to flood. However, raising access routes was considered very difficult, as many were major highways, and this would likely incur very high costs.

\subsubsection{Local Government}

LGAs will likely play a key role in adaptation to climate change, as most, if not all developers will interact with them at some time during the development process.

Many LGAs are currently amending their planning policies to address climate change. New amendments include: floodplain and bushfire risk overlays; requirements for setback and erosion controls; climate change rate changes; and compulsory use of rating tools. Some are also developing specific policies to manage, mitigate and adapt to climate change. For example, Brisbane City Council lists a variety of issues that climate change will impact, and that will require a planning response, including flooding, storm surge, Transport Oriented Development and incentives for sustainable development (Australia Green Infrastructure Council (AGIC), 2010).

While such changes have the potential to present a range of opportunities to the property development sector, they can also create a sense of uncertainty and confusion due to the rapid rate in which they were introduced; the inadequate level of consultation and the limited skills within government and the property development sector to implement the proposed changes (Local Government Association Queensland (LGAQ), 2012).

These issues were corroborated by respondents, who felt that dealing with LGA rules and regulations was often difficult, complicated and time consuming. The issue of time was striking throughout the research, but particularly in the context of LGA processes. These delays caused major problems for many developers as, "you make your profit when you buy and not when you sell", and, "time is money" (SD1, Small Developer). For example, one LGA took 7 years to approve a development application, and another developer said it would be 10 years before, "the first bit of ground was turned" on the development (MD3, Medium Developer).

Many reasons were given for delays, including "green" LGAs wanting to stop population growth by stalling development; others seeking to keep rates under control before elections; and LGA staff inability to assess innovative infrastructure. Another issue leading to significant time delays was disputes with the LGA, which could cost the developer significant amounts of money. During these often lengthy disputes, regulations sometimes changed, so the developer had to revise the proposal, costing more time and money.

62 The capacities of private developers in urban climate change adaptation 
Respondents felt that LGA capacity varied widely; most large LGAs were relatively sophisticated, but some smaller, regional LGAs were reactive, inexperienced and inadequately prepared for increased development in regional areas. The difficulties in attracting qualified staff to the regions was seen as contributing to this. Further, planning decisions were often dependent on the specific officer assessing the application, and many regional LGAs had high staff turnovers; one developer dealt with five assessment officers for a single development. A possible solution for these issues was increased education of LGA staff, and demonstrations about innovative projects in other areas.

Many LGA planning schemes were thought to be too complex, inflexible and attempting to duplicate State policy. In some schemes, areas were deemed suitable for development, but actually developing some sites was impossible, because they were constrained by multiple overlays, or conflicts with State policy. Nevertheless, many developers and consultants relied on LGA data and mapping to inform land purchases; by investigating the strategic plan, constraint layers, flood lines, etc. However, a developer had no way of knowing whether the information was correct, or up to date.

The perceived inability of some LGAs to deal with the diversity of developers and developments was seen as a major risk. When assessing a development application, developers said LGA staff didn't like to make decisions that were not clear cut, or on alternative methods of doing things that were not covered by the Planning Scheme and Codes. According to one respondent, LGA staff wanted to, "tick boxes and not have to think about anything. It's planning by numbers" (SG1, State Government). Developers argued that their ability to deliver sustainability depended on certainty, and such planning does not deliver this.

Many LGAs were seen as promoting sustainability, but in reality, were unwilling to consider innovative development; either because they viewed it as requiring additional maintenance (thus costing more), or because they were looking for "any excuse" to stall development approval and force developers elsewhere (CS8, Consultant). Many developers and consultants were seen as forward thinking, but they noted resistance from certain LGAs who didn't want change: "Councils tend to...do nothing it's easier to do nothing, and stick to what we <the Council> always do. Keep in our comfort zone" (SD1, Small Developer).

For example, a developer spent a year trying to persuade a "green" LGA to approve an innovative Water Sensitive Urban Design (WSUD) pressure sewerage system, which would treat the development's waste and stormwater on site, and reuse this in parks and gardens. After extensive lobbying, including field trips for staff, the LGA refused to pass the system.

"We were <expletive deleted> over by council, so we are not of a mind to push through those types of things anymore. We had a project with great environmental outcomes, and council just stifled it. We won't do these types of innovative development any more, takes too long, and there's not enough money in it" (MD3, Medium Developer).

Nor did LGAs provide incentives for innovative or sustainable development. For example, headworks charges were the same no matter how many sustainable elements were included in the development. As one developer stated, "the market and councils limit going ahead with great ideas. Councils stifle innovation, and markets won't pay for it' (MD3, Medium Developer).

Nonetheless, certain local governments, such as Logan, Ipswich and Moreton, were seen as more proactive and open to innovative developments. In general, developers felt that LGAs could play a major part in adapting to climate change, as long as they were prepared to be proactive. 
They also thought that some LGAs required a greater capacity to deal with extreme events; although some were already doing so, asking for standards such as 1:200 instead of 1:100. More frequently, it was felt that LGAs assumed the BCA would protect them. Finally, although not commonplace, if developers successfully completed an innovative development, some LGAs would incorporate elements of the design into their standards.

\subsubsection{General Findings on Regulation and Planning}

Regulation and planning were highly significant issues to survey respondents and interviewees. Government regulations were ranked very highly by survey respondents. $50 \%$ of the ten most significant issues were regulatory risks, and ranked first (DA approval processes and time to process applications) and second (infrastructure and headworks charges). Other major regulatory issues were ranked fourth, fifth and sixth (Figure 13). All regulatory risks were ranked as 4 (important) or 5 (very important).

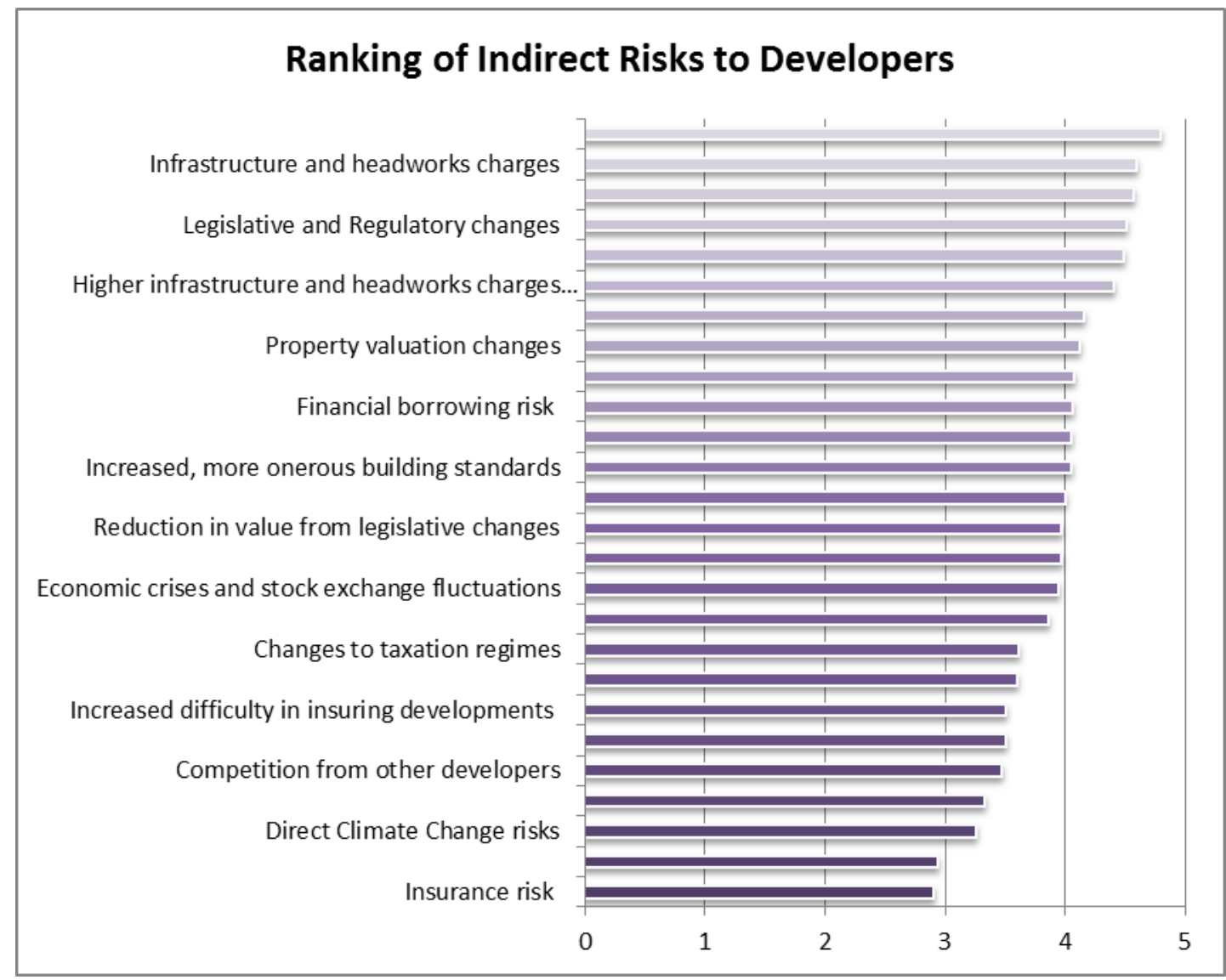

\section{Figure 12 Ranking of Survey Respondent Perceived Importance of Regulatory} Issues in Comparison with all Secondary Issues

Respondents felt that there was contradiction, confusion and duplication between planning instruments both horizontally (i.e. between local levels) and vertically (i.e. between Federal, State and Local governments). This lack of integration of various legislation and regulation was seen as a major issue. Some respondents also felt that the speed of legislation change was also a serious problem, and they sought more consultation.

Most respondents felt that more onerous regulation posed a major risk to them; and said that many smaller, "mum and dad" developers, had exited property development because they could not deal with the volume of approvals required. On the other hand, others felt that most developers understood well that regulatory risks were integral to 
any property development. Some larger developers even lobbied government, or used the PCA, to lobby government to change regulation they did not like.

Generally speaking, respondents felt that the industry was quite happy to follow regulation if they thought it logical, but not if, "you come out with crackpot things like the Coastal Plan" (SD2, Small Developer). However, different respondents held diverse opinions about the optimum levels of regulation. On one hand, some developers felt that virtually any regulation was too much, and that the market should decide the optimum product. To encourage innovation, they thought that rules needed to be freed up, and that government should stop trying to minimise every possible risk. In the words of a respondent, "you don't achieve better sustainability by regulating more" and "I have never seen a developer in 30 years who enthusiastically says, give me more regulation, it is giving me the incentive to do better" (SG1, State Government).

"Ok every so often you will get rid of the worst cowboy through regulation, you will prevent the worst excesses, but my experiences over time; society, industry, whatever you call it, weeds those out anyway. Their product doesn't sell. I don't know of too many shonky developers who are still in business after years and years and years. Their reputation is damaged. Having been in private practice you know the shonky developers. When they pick up the phone and say I have got a job for you, you say, sorry no work here at the moment. Because you know they won't build what you design, and you will be lucky if you ever get paid. And your name gets associated with a horrible product, and unless you are a shonky consultant yourself, and you are not in business for very long anyway...." (SG1, State Government).

On the other hand, some felt that although development practices had improved significantly over the past 20 years, and developments had become more environmentally sustainable, this has been largely driven by the regulating authorities. They also felt that developers would not do anything more than the minimum requirements of legislation and the BCA unless there was a distinct market advantage to doing so. Many had similar views to the respondent who said the recent change in government in Queensland meant that, "the regulatory stick is going to be put back in the cupboard for the next few years, so other drivers will have to be used" (SG1, State Government).

A common theme was that the planning process had become increasingly complex, confusing and contradictory; and that the supposed "improvements" to the planning process had not succeeded, and instead made planning more complicated. For example, a respondent stated, "The complexity of the planning process has robbed a generation of planners of their ability to exercise fundamental judgement about what is good or what is bad" and "Some planners said they feel they lost the plot years ago what planning was supposed to be achieving and what it was all about" (SG1, State Government). Although the government had tried to set up rules that "even monkeys could follow", the planning process required intelligent engagement from assessment officers (SG1, State Government).

The plethora of "red and green" tape, the increase in checks and balances, and the requirement to produce extensive studies and reports was seen as extremely onerous and time consuming. One developer felt that Information Requests received from LGAs or State Government often reflected that the assessing officers had not even read the expensive reports they had requested. Some State Government departments, such as the Department of Environment and Heritage, were seen as particularly problematic in this regard, requiring extensive studies for relatively minor issues. 
"Regulation changes all the time. A lot is necessary, but a lot of it is just dumb and filling forms for the sake of filling in forms, and spending on consultants not necessary. Politicians are just there to make rules" (SD2, Small Developer).

Developers also felt that issues with regulatory agencies often resulted in long periods to get developments approved. Government structures were seen as barriers to efficient development processes, because of fragmentation and silos within agencies and different, sometimes contradictory legislation. Improving consistency between agencies was seen as vital. In addition, respondents often had difficulty locating public servants with sufficient delegated authority to sign off a development approval. Respondents also felt that many assessing officers were confused by some developments, and applications were often passed from officer to officer. Further, there was a lot of churn amongst assessing officers, and planning advice changed with each new officer. A developer discussed the fast-tracking of the Grantham rebuilding:

"You know what the crazy thing is, normally this process would have taken us 2-3 years, it took them 6 months...exactly the same outcome" (SD1, Small Developer).

Another theme was the desire for more certainty from planning processes. Participants thought it was very difficult to do the preliminary work for a development without certainty from the regulators. A common opinion was that they had to work within a system that didn't know how to make decisions on things that were uncertain. Further, many wanted more accountability and transparency from regulators; as well as greater flexibility.

"The legislation gives uncertainty, but the developer also wants

flexibility... and this creates tension" (CS2, Consultant).

Many expressed the opinion that there was a lack of flexibility in government planning processes and codes. Government agencies wanted fixed information such as yield and number of lots, but this caused problems with large, staged projects. For such projects, the developers wanted flexible approvals, so they could change aspects of a development depending on variables such as market demand and future transport nodes.

Therefore, some said they just gave very broad assumptions to government requests for detailed information. They felt that it was impossible to predict, for example, higher level issues (such as future use of public transport), as there were so many variables that could influence this. On one hand, respondents wanted certainty that the government would not move the goal posts, but on the other hand, they also wanted flexibility of approval. Thus, many felt that planning needed to be streamlined, not by corner cutting, but by ensuring that things were done more efficiently.

One way in which developers dealt with the issue of "excessive legislation" was to move to another area. They tended to develop where it was simplest, as many developers were relatively mobile, and even moved interstate to source cheaper land, or to escape onerous regulations.

A potentially significant issue for adaptation to climate change was that developers relied on planning agencies to have accurate data and provide correct information. Related to this, was an overreliance by insurers, bankers and developers that the planning authority and LGAs had done their due diligence and their risk modelling was correct. However, in many cases, government mapping is conducted at relatively large scale (1:100,000 scales or larger), and base information is sourced from the grey and not the academic literature. Concern was also expressed that government had no real understanding of the implications of climate change, especially if some of the higher level models proved accurate. 
"You set up a system where the inputs to decision making that specified by the system, are not the sufficient to inform the inputs, so if you rely on that, you are on very shaky ground" (SG1, State Government).

Many developers felt that changes in regulation and legislation regarding climate change were happening very quickly. However, they felt the issue was more with the legislation around climate change, than climate change itself. Most developers did not think climate change was particularly important, and nor were they interested in the actual issues of climate change. Some respondents expressed the opinion that adapting to climate change was nothing more than complying with the regulations. However, it was necessary for assessment agencies to find a middle ground, or they would be taken to task by both developers and the environmental sector.

A smaller number of respondents thought climate change required a distinct policy on its own, and that dealing with it as part of SPA or the Coastal Plan was inefficient. Some also stressed that climate change and all the concomitant regulation was too confusing, and there were too many funders, levels of government and agencies dealing with the issue; and that there should be one integrated agency (champion) to drive the cause.

The change of government in Queensland was seen to have implications for climate change (and other environmental) regulation. One consultant felt that the Newman government was cutting every "green" program, and a huge amount of change and shifting accountabilities was evident. Further, many projects, i.e. a green infrastructure project, of which a component is climate change adaptation, had been negatively impacted by government withdrawals and lack of funding support from State and Local Government.

Some developers also thought the change of government meant that now (October 2012) was a good time for developers to start projects or put in applications, because the new State government was pro-development, LGAs wanted to encourage economic growth and the market was still flat. One planner stated that the current government wanted to preserve the best of the planning element but to remove the process elements, which were just "red tape".

Many felt that if climate change adaption regulation were to be introduced, there would not be resistance from the industry, particularly if the regulations were worthwhile and logical. It was also considered important that planning guidelines for adaptation should be clear, and incremental. However, others thought that private industry (developers, insurers and bankers) would be the drivers of change, and government would follow:

"Many in industry understand sustainability; they build well oriented houses, with water tanks, solar, etc., they do this without regulation forcing them to do so" (SG1, State Government).

However, some large and powerful developers reportedly lobbied government ministers directly (or indirectly through the PCA) to thwart any attempts to introduce what they considered onerous regulations about climate change. Because these firms knew they could shift the politics by donating money to government, they felt regulation changes did not pose a major risk. Even smaller developers had factored a change of government into future plans, deciding to persevere with a development because they saw a conservative government as supportive of development.

Despite this, nearly all respondents felt that improved cooperation between government and developers would give better outcomes. Developers saw themselves as constrained by time, money and government (regulation). If government did not allow them to be innovative, by encouraging flexibility and reducing red tape, then they would not embrace innovation. 
However, some felt that industry would not be able to deal with the issues raised by climate change, particularly given the uncertainty in the market. Even though large consultants were gearing up to deal with climate change, they observed very little leadership from government on practical methods to achieve this. Further, it was widely assumed that government did not understand that developers were business people, and their primary motivation was to make profit; and any regulation that potentially impacted on profitability was viewed extremely negatively. Finally, some commented that it was important to spend money on research, especially on how business and government could work together to adapt to climate change.

\subsection{Industry Self-Regulation}

In addition to government legislation, many property developers, financiers and industry bodies have developed their own ratings and certification schemes. In essence, a developer will voluntarily implement policies and measures to improve the environmental (and social and economic) sustainability of a development.

Numerous development companies promote social and environmental goals. For example, some UK development companies have integrated social and environmental performance into their core business models to ensure their products achieve high standards (Bryson \& Lombardi, 2009). Certain Australian developers are also noted for producing high quality developments, which are both economically profitable and socially supportive (for example, Land Lease and Mirvac). These organisations include, in their corporate material, multiple references to achieving sustainability.

Various voluntary industry-specific codes, standards and conglomerations attempt to address the environmental and social impacts of property development. These include reporting tools such as Corporate Social Responsibility (CSR); the Green Star and the National Australian Built Environment Rating System (NABERS) rating systems; and the EnviroDevelopment certification scheme. These are commonly used in commercial development, and to a lesser extent, in residential development.

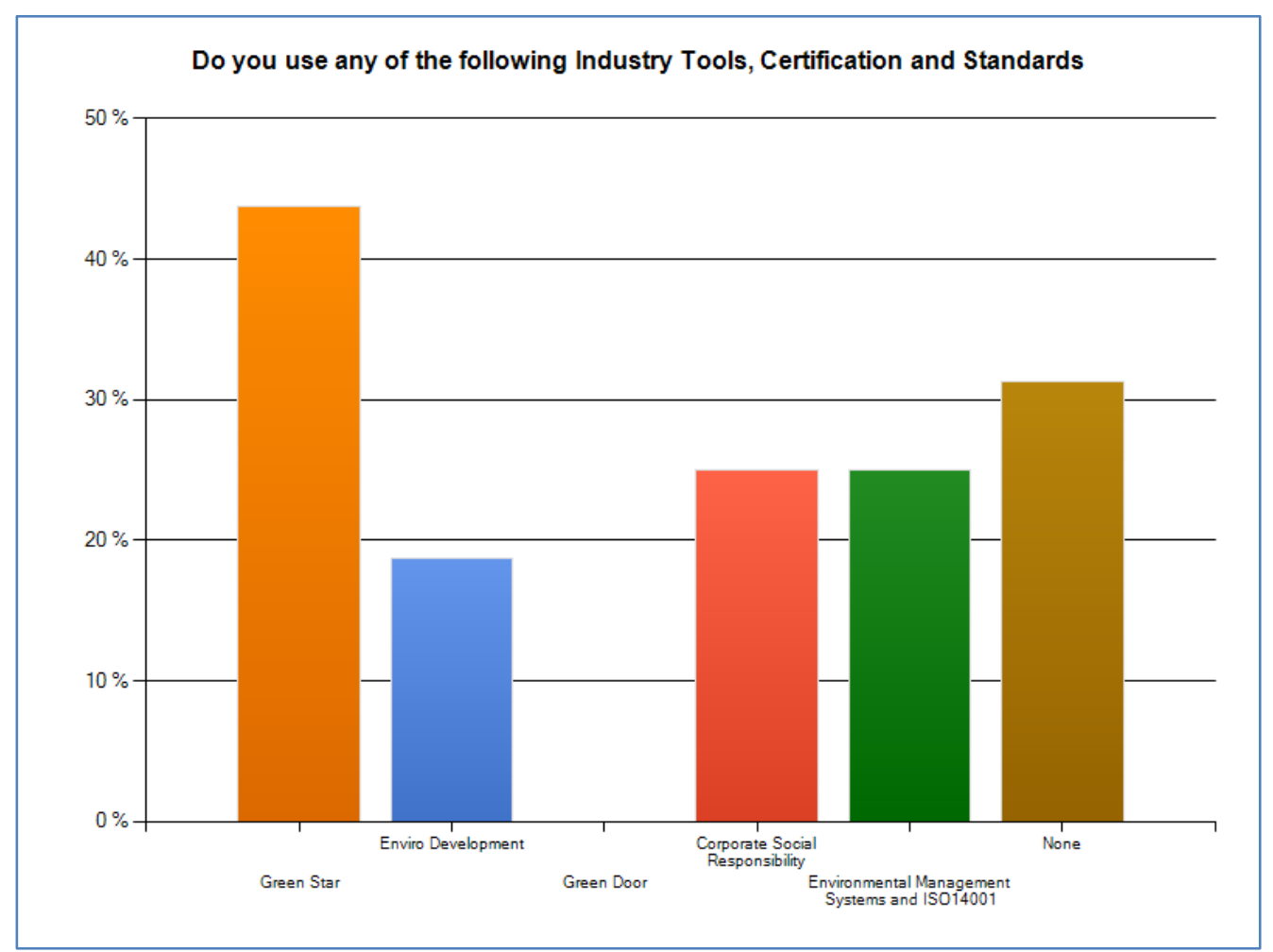

Figure 13 Percentage of Survey Respondents using Industry Self-Regulation Tools (note: respondents could select multiple choices)

68 The capacities of private developers in urban climate change adaptation 
As indicated in Figure 14, almost half of survey respondents $(44 \%, n=14)$ used Green Star; a smaller percentage used CSR and EMS $(25 \%, n=8)$, and $19 \%(n=6)$ used EnviroDevelopment. Approximately one third $(31 \%, n=10)$ used no self-regulation tools. There was no significant difference between category of respondent or type of development with regard to industry self-regulation.

\section{Corporate Social Responsibility, Legacy and Branding}

CSR is broadly defined as the implementation of activities that addresses environmental and social issues 'beyond' regulatory compliance and a company's interest to maximise its profits (McWilliams, 2006; Prakash, 2001). It is generally accepted that CSR 'goes beyond' compliance, but the extent to which it supersedes profit maximisation goals, is debated (Decker, 2004; McWilliams, 2006). Many companies that implement CSR standards and practices do so hoping to reap some benefits, usually by capitalising on new business opportunities or enhancing their reputation.

CSR is not a new concept in the property development and development finance sectors. Many development firms, or allied industries, were active in the social responsibility field relatively early; for example, Weiss (1987) argues that much of what are considered as good planning principles didn't come from planning, but are principles that were worked out on the ground by 'good' developers.

No respondents specifically mentioned CSR, although many referred to social responsibility, and corporate reputation. To some extent, CSR was analogous to the terms "legacy" and "brand". Developers were extremely concerned about their reputation, and desire not to be seen as "part of the white shoe brigade" (in reference to the past "cowboy" developers of the Gold Coast) (SD1, Small Developer). A common theme was a desire to counter the perceived public opinion of developers as unprofessional; "there's a perception out there, we've got a bad legacy" (SD1, Small Developer).

"Developers take on a very significant risk to deliver things to the community and I don't think it is something that is recognised. That role in the community. They see us as pretty evil, evil corporations trying to screw them, and we take on pretty significant risks to achieve these things" (MD3, Medium Developer).

Larger developers were particularly concerned about their brand and legacy. Larger developers had corporate reputations to uphold, as well as responsibilities to their shareholders. Some also spoke of having a community responsibility, to ensure that their developments were socially as well as economically and environmentally sustainable.

"They [large developers] don't want to build these new developments of 4,000 to 10,000 people that are going to be vulnerable to extreme events because that will have an impact on their brand and their legacy that they are leaving" (SC1, Sustainability Consultant).

\section{EnviroDevelopment Certification Tool}

This tool was designed by the UDIA in an attempt to address the increase public and industry awareness of environmental concerns and the public demand to uptake sustainable development. The tool is defined as; '[a] scientifically-based branding system designed to make it easier for purchasers to recognise and, thereby, select more environmentally sustainable developments and lifestyles' ((UDIA), 2011). The EnviroDevelopment framework has been structured to encourage innovation and incentives for incorporating sustainable development principles in residential, commercial, industrial and multi-use developments across Australia. 
The EnviroDevelopment framework offers six categories of certification (with a "leaf symbol" for each category of accreditation) (Figure 15). These leaf symbols include: water (improved water efficiency); energy (energy efficiency, and reduced GHG emissions); ecosystems (protection of environmental sustainability and native biodiversity): community (cohesive, sustainable communities, with good networks, public transport and affordable housing); materials (reuse, recycle and lifecycle considerations); and waste (waste management practices and procedures).

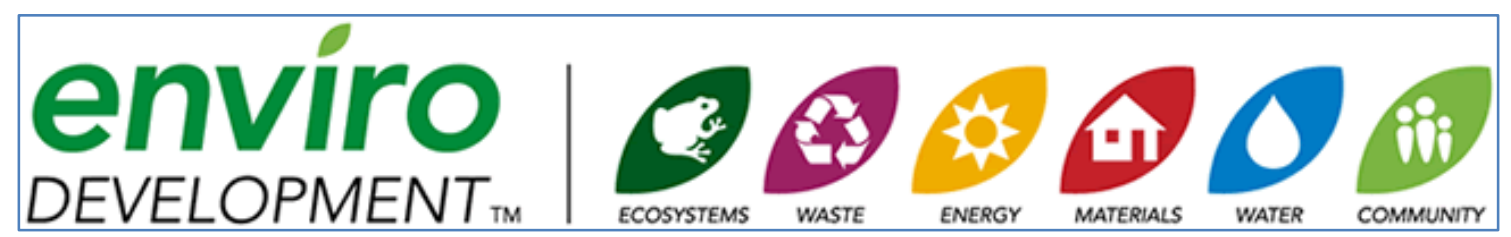

Figure 14 EnviroDevelopment Logo showing Leaf Symbols (Source: http://www.udiawa.com.au/go/industry-envirodevelopment)

EnviroDevelopment was used by many participants in the study; and was generally viewed very favourably. Some became involved in EnviroDevelopment because they wanted to do the right thing for the environment, while others nominated it as their primary source of information on sustainability. Of note, the majority of the interview participants were sourced from those who had participated in the UDIA

EnviroDevelopment framework, so these findings should be viewed with some caution. Approximately $20 \%$ of survey respondents also used the framework (Figure 14).

One medium sized developer said they thought that if they didn't self-regulate, then the government would come in over their heads and do it for them. Others mentioned that they joined EnviroDevelopment primarily for the marketing advantage, but also to do the right thing, and to stay ahead of the constantly changing regulation.

\section{Green Star}

Green Star is an environmental rating and certification system devised by the Green Building Council of Australia (GBCA 2012). Green Star is mostly used for commercial and industrial buildings, but it also incorporates multi-unit residential buildings. The Green Star tools can be used from the design phase to the end of construction. The tool rates each building under nine weighted categories; Management, Indoor Environment Quality, Energy, Transport, Water, Materials, Land Use and Ecology, and Emissions. Each category is further broken down into sub-categories and the tool also includes metrics such as proximity to public transport, and indoor air quality.

Green Star was the most commonly used industry scheme, with almost $50 \%$ of survey respondents using it; and it was frequently mentioned in the interviews and focus groups (Figure 14). Green Star ratings were seen as beneficial for commercial properties, and developers used it as a market tool to get quality tenants. For example, State Government agencies and some large corporates demanded minimum Green Star ratings before they would consider renting premises in a commercial building. A major bank stated that the main reason they chose a specific building for their corporate offices was the factor 6 Green Star Rating (FI2, Financier). The demand for "greener" office buildings was a major push factor in this market, and developers even competed with each other to produce higher rated buildings.

"There is a very good lesson in green star. Don't get a premium, but you get quality tenants. You are not going to do this to say why are my margins going up, because they do, but maybe if you take a long term view and say that your asset value is going to be maintained" (SD1, Small Developer). 
Attitudes to Green Star were largely positive, although one developer said that such rating scales constantly changed, and it was necessary to "lock in" developments at a certain level. Further, it was felt that there was a "law of diminishing returns" in rating systems, with the higher levels costing significantly more money. The differences between, for example 7 and 5 star rated buildings, were relatively minor, and customers may not be willing to pay the higher prices.

By contrast, Green Star (and other rating systems) was not as commonly used for residential buildings (apart from some high-rises). However, there was virtually no market demand for higher rated residential buildings, largely due to perceived higher costs and little future benefit. This is related to the timescale of developments; residential developers usually "get in and out" as quickly as possible, whereas commercial developers tend to keep the asset and lease it out. As higher Green Star ratings reduced operating and maintenance costs of the building, this was seen as a major benefit.

\section{NABERS (National Australian Built Environment Rating System)}

NABERS is often used in concert with Green Star ratings (NSW Office of Environment and Heritage, 2012). It seeks to measure environmental performance of a building by measuring energy efficiency, water usage, waste and indoor air quality. It uses information such as utility bills and converts these into a star rating from 1 to 6 stars. It seeks to rate residences as well, but like Green Star, is mostly used in commercial or retail buildings.

Rating systems such as Green Star and NABERS are widely used in Australia, and have the potential benefit of normalising environmental (and thus climate change) issues in construction. Common to such systems is a relatively rigorous and weighted checklist to estimate the overall environmental rating of a building (Nalewaik and Venters 2009). Further, such ratings can offer tangible, measurable benefits; including lifecycle cost savings (to developers and home owners); design savings; improvements in construction process; and the "feel good factor" (Nalewaik \& Venters, 2009).

These non-regulatory tools appear to offer the potential for major opportunities from a changing climate. The overall cost-benefit is favourable, and they can potentially save money in design and construction, and even mitigate some GHG emissions. In some cases, developments using these rating schemes can also be marketed at a higher price, as "green", therefore increasing the profit from the development.

The major point of difference between developers using rating systems was that commercial buildings are generally kept for the long term and leased to tenants, whilst residential buildings are sold. If developers kept their commercial buildings, they wanted two things, to attract the best quality tenants, and to reduce operating costs. Systems such as Green Star and NABERS could achieve both goals.

A major driver for introducing sustainability rating systems was demands by quality tenants (such as Government agencies or large corporates) for higher rated buildings. In addition, Brisbane City Council also introduced an incentive scheme for certified buildings, with developers paid a rebate.

"It was through the Brisbane City Council. They had the rebate scheme that said if you have a NABERS building, we will give you a value of money, for that contribution towards getting that rating, per square meter, depending on your contribution. That actually signalled to the market so everyone including Leightons, and they had quite a few commercial buildings at the time, went and pushed to get that rating, because they got about $3 / 4$ of a million dollars for one of their buildings. That actually drove it" (LD2, Large Developer). 


\subsection{Conclusion}

Regulatory changes are currently occurring across all levels of government in Australia. Some examples include; the introduction of the Carbon Tax and the requirement to consider climate change in SEQ planning schemes. Regulatory changes may also come from within the industry or from associated institutions, such as lenders and insurance companies (discussed in the following chapter). These changes are dynamic and in theory, are aimed at supporting climate change adaptation and mitigation efforts. However, challenges for developers can rise from the inflexibilities or inefficiencies of existing regulatory mechanisms, or changes to regulation.

The implications of changes to the policy and regulatory framework for developers could be significant, ranging from increase in the cost of development to permit refusal and legal challenges (Briggs et al., 2010; Taylor et al., 2012; Urban Development Institute of Australia (UDIA) South Australia, 2011, p. 3). A key challenge associated with the dynamic nature of the regulatory environment is the uncertainty surrounding the introduction of new policies and regulations.

Very few respondents commented on Federal legislation and regulation, including the Carbon Tax. However, most respondents had (mostly negative) comments about State government and LGA regulation. The most criticised legislation was the Queensland Coastal Plan (now partially suspended by the Newman LNP government).

State Government was criticised because of multiple, complex regulation, and lack of integration between the different government agencies. It was felt that the multiple processes introduced to simplify planning during the last 20 years had not succeeded. Planning and legislation had become ever more complex, but its assessment had been reduced to a tick box system of "performance-based" codes, which did not reflect the diverse and complex environment of developers and development.

LGAs also received criticism, but this partly depended on their size and sophistication. The larger LGAs were viewed relatively favourably, but some smaller LGAs were widely criticised. The major criticism of LGAs was the time they took to approve developments. In some cases, LGAs had stalled development approval for longer than a decade. These time delays had cost the developers large amounts of money, and in many cases, had forced them to move their business elsewhere. Some felt that the reason for this was that LGAs would not approve innovative developments, as LGA officers were unable to assess developments that did not adhere to their codes. LGAs were seen as paying lip service to sustainability, but in reality, stalled sustainable developments that required more maintenance. Many LGA staff were considered relatively unsophisticated, particularly in regional areas. This was partly due to the "churn" of planners, because of staff poaching by the resource industry.

Different respondents held diverse opinions on regulation. Some felt that most regulation was unnecessary, and that the industry and the market should drive innovation and adaptation to climate change. On the other hand, others felt that developers would not adapt without the "regulatory stick", and it was essential that government drove any changes. However, most felt that government should introduce climate change legislation incrementally, and that any legislation introduced should be logical and practical. Nearly all respondents wanted government to consult more.

Industry self-regulation appears to be a potentially valuable driver for sustainability and increased adaptation to climate change. However, at present, most rating systems are used only in commercial buildings and not in residential development. The demand for these is driven by tenants, government subsidies and reduced operating costs. Only the largest developers introduce major sustainability initiatives into residential developments, and all reported that there is no demand for "green" buildings from the residential sector. 


\section{FINANCE AND MARKET}

\subsection{Introduction}

"They are in this to make money in the short term and that what the property development sector does...end of story" (SC2, Sustainability Consultant)

The role of financing is integral to property development, as the majority of developers are reliant on external funding. However, the interrelation of funding and development is a complex process, and is continually changing along with economic conditions.

Therefore, financial or economic risk is one of the primary sources of risk to the property development industry. Financing development can be extremely risky, as it is influenced by a range of external and dynamic factors such as local and global economic and market conditions. Regulatory changes introduce a new dimension to financial risk; that of time. Arguably, for all developers, "time is money" and the longer it takes for a development to be approved, the more it can cost.

Multiple sources of development finance exist apart from traditional lenders, such as banks. These include insurance companies, superannuation funds, internal funding, property companies, the construction industry, government, not for profit organisations and private individuals. Further, the process of securitization-the process of transforming an asset into a security, to secure further borrowing-has allowed new types of organizations to become involved in development finance, by giving access to longer term funding, and aiding the evolution of global conglomerates (Logan, 1993).

Climate change may add further elements of risk to borrowing by introducing additional physical and regulatory hazards. Physical hazards include the cost of the direct impacts of climate change; and regulatory changes are those that are implemented by governments or by different economic sectors. These changes could influence lending decisions and conditions. For example, some lenders may not be willing to fund developments in areas exposed to risk, and others may impose onerous requirements on the developer.

The insurance industry is a source of further risk when financing and marketing property. Extreme weather events, such as cyclones, are forecast to increase in intensity due to climate change (World Bank, 2012b, p. 16). The insurance industry in North Queensland has already raised premiums since Cyclones Larry (2006) and Yasi (2011). In some areas, even those which suffered little or no cyclone damage, such as Townsville and Mackay, some premiums have risen over 300\% (Insurance Council of Australia, 2012). This has particularly affected strata titled unit and townhouse complexes, as body corporates have to insure not only the property, but also common areas. In some regions, prices of unit complexes have dropped significantlysometimes by nearly $50 \%$-due to depressed economic conditions and higher body corporate fees from to increased insurance premiums (Australian Government Actuary, 2012; Studdert, 2012).

\subsection{GFC and economic climate}

Property development in Australia used to be primarily a small-scale activity, conducted by local firms in small markets. However, in the early 1990s, new ways began to emerge how firms raised funds on the global capital market (Logan, 1993). This is known as 'securitization'; converting an asset, such as real estate or mortgages, into a financial instrument that can be traded on financial markets (Logan, 1993, p. 8).

Securitization enabled new types of organizations to become involved in development, by giving access to longer term funding, at lower costs and with less direct intervention and control by lenders. This led to the development of large conglomerates, many of

The capacities of private developers in urban climate change adaptation 73 
which carried out their own funding operations; and financial institutions which carried out property development. This also changed the capacity of developers, enabling them to undertake larger and longer term projects, and to spread their risk across multiple projects and sub-markets (Coiacetto, 2007) and across the vicissitudes of economic cycles (Logan 1993, p.49).

Arguably, the process of securitization was a major contributing factor to the Global Financial Crisis (GFC) between 2008 and 2009 (Allen \& Faff, 2012). Property prices in the USA (and Australia) had dramatically increased from the early 2000s until late 2007. This was related to a form of lending called "subprime" mortgages: borrowers, who previously could not qualify, were granted loans, sometimes greater than $100 \%$ of the value of the property. These loans were then bundled up and resold to investors, in the assumption that continuing capital growth would make up for their high risk nature. However, the bursting of the "property bubble" in the USA caused a cascading effect around world markets. House prices dropped dramatically, with the consequence that many of these borrowers defaulted on their loans and the value of the securitized assets plummeted (Allen \& Faff, 2012; Harper \& Thomas, 2009). Because of this, many lending institutions either went bankrupt, or had to be "bailed out" by governments.

While Australia had less exposure to subprime mortgages than the United States, property prices also had risen dramatically since 2000; in some cases, more than doubling. This was partly due to the economic flow-on effects of the Sydney Olympic Games, and the concomitant rise in house prices. It was also partly due to the Howard Government's institution of a generous First Time Homebuyers Subsidy. As Sydney house prices rose, many sold their houses, and bought in areas where the prices had not yet risen, such as SEQ. The subsequent population growth in these areas was dramatic; over 4\% in some Queensland LGAs (ABS, 2009).

The SEQ property development industry profited significantly from the increased investment and population growth. There was a huge demand for property, particularly from interstate purchasers, who could sell a small 3-bedroom house in a "bad" suburb of Sydney and purchase a 4 bedroom house in a "good" suburb of SEQ. Because of this demand, property development activity flourished, and high profits were almost guaranteed from capital growth.

Now, in addition to established property developers, new players, eager to profit from the boom, embarked on property development. Many had no experience in property development, and little equity. However, the lending institutions were almost "throwing money at developers and buyers"; often at very high Loan to Value Ratios (LVR) (SD1, Small Developer). Because of sustained capital growth, the risk to the developers, and the lenders was relatively minor. Speculation was rife, with buyers happy to buy off the plan, at relatively high prices, because they "knew" they would make a profit on resale.

However, no market rises forever, and all property booms eventually end. The GFC had an all-encompassing and radical impact on the property development industry. Some regions were impacted more severely than others, particularly areas like the Sunshine Coast, with a larger proportion of high risk industries, such as building construction. Regions which had high percentages of new developments, such as the Gold Coast, were also severely affected. Other regions, such as Cairns and Airlie Beach are heavily dependent on tourism. The combination of the GFC, the high Australian Dollar and Cyclones Larry and Yasi, had a severe impact on their economies. In general, if an area had a strong economy, such as Brisbane, it was better able to survive the financial storm (i.e. the GFC) and the climatic storm (i.e. the 2010/2011 floods). 
No matter how strong the local economy, the GFC still made development very difficult. During and after the GFC, many developers and consultants declared bankruptcy, exited the market, or laid off staff. Firms that were highly geared (i.e. owed more money and did not have the cash flow to service this) generally did not survive the economic crisis.

"Through that post GFC situation the market pretty much crashed. So broadly speaking there was no market; purchasers weren't purchasing, if they signed a contract they weren't settling. Generally speaking, people only settled if they had to, a lot of them resorted to legal manoeuvring to try and get out of some contracts" (FI1, Financier).

Although the GFC ended in approximately 2009 , the property development sector has not fully recovered. Many bigger developers have postponed starting some projects, and are just "riding it out" until the market turns and there are more margins in development; and the banks begin easing lending policies. Consumer confidence remains low, with the result that the development sector is relatively stagnant.

Many respondents felt that, by now, the market should have recovered to a greater extent. Some economic indicators, such as new home approvals, have begun to improve; however, as a small developer stated:

"There was a lot of stock on the market and a lot of projects in the pipeline that just couldn't be finished, the developer had gone broke. Because we had a long party, the hangover is over, and it is the longest I've seen"(SD1, Small Developer).

\subsection{Sources of Finance}

Development is risky, and expensive. A developer places a considerable investment of financial resources at risk. Therefore gaining access to funding is critical for developers, and for development to occur. Due to the risk involved, gaining access to development finance is traditionally problematic.

In the past, most developers were local businesses who would raise money for a project by applying to a lending institution, typically a bank (Logan 1993). These still make up the majority of smaller developers. Any application for funding is assessed against the potential range of risks it poses to the lender, including contractor risk, site risks, planning risks, borrower risk (e.g. track record and management capacity), and market risk (Coiacetto 2012, 56-7). If successful, the developer obtained a loan for a specific project, such as land acquisition or construction. The loan was generally shortterm, the interest rate included a margin for perceived risk, and was subject to conditions such as site inspections and progress reports.

Since the 1990s, numerous additional sources of funding exist, but change regularly, as they are influenced by changes in the economy and other factors. In 2007, prior to the GFC, the market was dominated by the four major Australian banks (e.g. Westpac, ANZ, Commonwealth Bank and National Australia Bank), followed by regional Australian banks and foreign banks, mortgagee trusts and other sources of finance (Ashe Morgan Winthrop, 2011). Other sources of finance included insurance companies, superannuation funds, internal funding, property companies, the construction industry, government, not for profit organisations, private individuals, international private equity funders, and securitization.

Since the GFC had a significant impact on the sources of development finance. The financing market underwent a number of changes in both the source of the funding (e.g. banks, insurance companies, etc.) and in the form of the funding (e.g. mezzanine and equity funding) (Bryant, 2012). The number of funding institutions has been significantly reduced post GFC (from approximately 40 to just three or four major 
lenders) (Bryant, 2012). So risky was property development finance that a medium sized bank, highly active in development lending, almost went bankrupt, before the Federal Government intervened (FI2, Financier).

An additional consequence of the GFC was that conditions under which developers can gain funding have become much more restrictive (Ashe Morgan Winthorp 2011; Bryant 2012). Lending institutions have again become extremely conservative and risk averse, particularly about high risk lending, such as for property development.

Therefore, the process of development funding has to some extent, reverted back to pre-1990s, with 'traditional' developers sourcing funding from major banks, with multiple conditions attached to the loan.

Table 5, adapted from Bryant (2012; and Ashe Morgan Winthrop, 2011) outlines how key lending criteria (i.e. developer, sector, location and gearing) were modified during and post GFC for development in Queensland Australia.

Table 5 Changes in key lending criteria post GFC (Adapted from Bryant 2012 and Ashe Morgan Winthrop, 2011)

\begin{tabular}{|l|l|l|l|}
\hline $\begin{array}{l}\text { Key Lending } \\
\text { Criteria }\end{array}$ & $\begin{array}{l}\text { Pre GFC } \\
(<2007)\end{array}$ & $\begin{array}{l}\text { During GFC } \\
\mathbf{( 2 0 0 7 - 2 0 0 9 )}\end{array}$ & $\begin{array}{l}\text { After GFC } \\
(\mathbf{2 0 0 9 )}\end{array}$ \\
\hline Developer & Almost anyone & $\begin{array}{l}\text { Only very } \\
\text { experienced }\end{array}$ & Experienced \\
\hline Development Type & $\begin{array}{l}\text { All, some lenders } \\
\text { specialised in } \\
\text { certain sectors }\end{array}$ & Mostly residential & $\begin{array}{l}\text { Mostly residential } \\
\text { until recently, now } \\
\text { some commercial }\end{array}$ \\
\hline Location & All Queensland & $\begin{array}{l}\text { Major centres or } \\
\text { SEQ only }\end{array}$ & $\begin{array}{l}\text { Mostly metropolitan } \\
\text { or resource areas }\end{array}$ \\
\hline Gearing (\% cost) & $80 \%-85 \%$ & $0 \%-70 \%$ & $60 \%-75 \%$ \\
\hline $\begin{array}{l}\text { \% of Completed } \\
\text { Value }\end{array}$ & $>75 \%$ & $?$ (likely $<65 \%)$ & $65 \%$ \\
\hline Pre-Sales & $0 \%-60 \%$ & $100 \%-110 \%$ & Min. 75\%-80\% \\
\hline Equity & $0 \%-20 \%$ & $25 \%-100 \%$ & $80 \%-100 \%$ \\
\hline
\end{tabular}

The results of Bryant's analysis demonstrate the following post GFC trends: (1) major lenders favour lending money for residential development projects as opposed to commercial and industrial projects; (2) lending institutions favour prime metropolitan locations over regional locations;(3) gearing and presale conditions have become tougher and developers are required to have $>25 \%$ equity, generally using the land as security;(4) the size of the deals in terms of monetary value have declined; and (5) lending fees have increased (Bryant 2012).

In this study, the majority of smaller developers obtained funding from the major banks, but there was a significant diversity of funding sources. Notable differences between the size of developers and consultants and funding sources were apparent. For example, only medium and large developers noted cash as a funding source, whereas smaller builders and consultants apparently obtained funding from many sources. Developers also accessed a diverse range of other funding sources, such as minor banks, high net worth business partners, family trusts, private equity, government grants, NGOs and Superannuation funds (Figure 16).

76 The capacities of private developers in urban climate change adaptation 


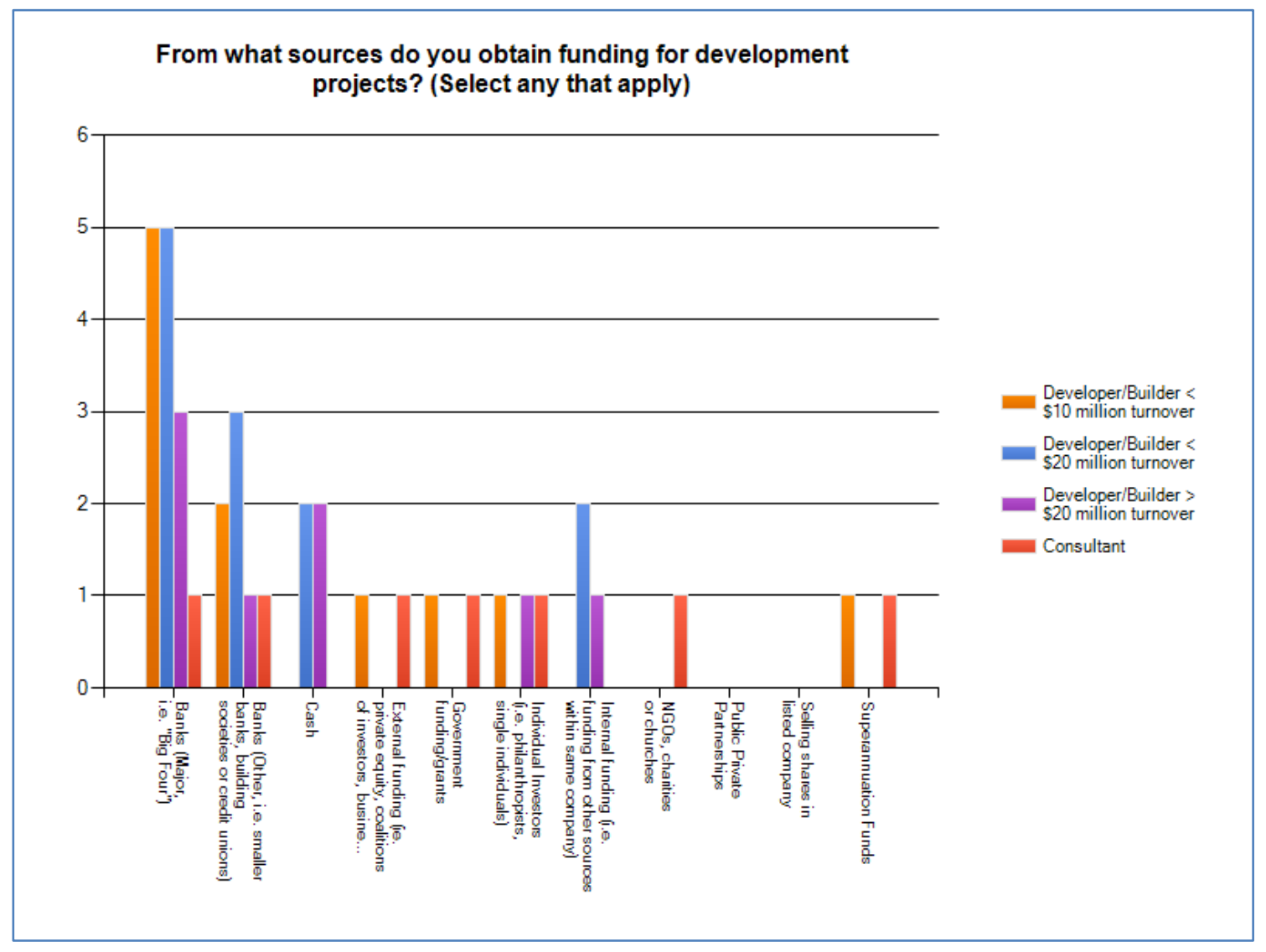

Figure 15 Sources of Funding for Survey Respondents

Since the GFC, banks have imposed more onerous conditions on development funding. Only if a developer had a good relationship with their bank, and ample equity and capital, then would the bank consider lending them money. Typical conditions included: lower LVRs; a higher percentage of security for the loan; and the requirement for a minimum of $75 \%-80 \%$ (and in some cases, $100 \%$ ) of pre-sales.

As a developer said: "Banks say they are lending again, but they have pretty high requirements, especially in pre-sales; want $100 \%$ debt cover in the form of pre-sales" (SD1, Small Developer).

This concurs with Ratcliffe et al. (2009, p.430) where the key factors that determine the source of finance are: (1) 'the status of the developer', and (2) 'the degree of risk attached to the proposed project'. Since the GFC, banks have weighted the degree of risk to a much greater extent; developers and consultants were almost unanimous in highlighting an extreme shortage of funding, and felt that obtaining bank finance was one of the biggest hurdles for developers.

Banks were also unwilling to take any planning risk, and refused to finance developments if planning approvals are not already in place. Further, banks were not willing to fund certain areas, as there was seen to be no demand; i.e. high risk apartment buildings on the Gold Coast, where there was a major oversupply of stock.

Because of the difficulty in obtaining bank finance, some developers reported accessing private equity funds from overseas; or attempting to do so. Mezzanine finance was available, but it was significantly more expensive than bank finance.

"Overseas finance means investors from overseas who have big bundles of money and they want to park it somewhere. Australia is very politically stable and a good place to put their money. Almost no bank finance. They are just not lending" (CS4, Consultant).

The capacities of private developers in urban climate change adaptation 77 
Not all developers were reliant on bank finance. Large publicly listed developers or consultants funded their projects from different sections of their business. For example these used more secure businesses (i.e. a commercial property leased to long term tenants) to fund riskier businesses (i.e. residential development). Consultants were largely funded for providing consultancy services to developers and government. Other developers obtained finance from high net worth business partners, or other private equity sources. No participants obtained funding from superannuation funds or charity/NGOs.

Allied to the problem of obtaining bank finance, were issues with property valuation. Valuers often collaborated with banks, so were viewed as working against a developer's best interests. Some said that valuers were undervaluing properties due to lack of market demand, and at amounts they knew banks would recoup if there were a "fire sale".

\subsection{Market Demand}

Aside from finance, possibly the most serious risk faced by property developers is market risk, because it occurs after the developer has invested resources in the project. The most difficult challenge is to anticipate the state of supply and demand at the completion of the project. The broader economy moves through unpredictable boom and bust cycles, and the supply and demand for development products is in continual flux. Consumer demand in different market sectors and submarkets is affected by external factors such as interest rates, fashion; and demographic and economic changes. In addition, projects by other developers may also come on to the market, and these could oversupply the anticipated demand. Moreover, residential markets are highly segmented and each submarket, such as second home-buyers or investors, presents its own risks and opportunities which require different competitive strategies (Coiacetto, 2007).

In this study, most participants felt that the major concern of the banks when deciding to loan money was the percentage of pre-sales, i.e. the market demand for their product. Further, consumer sentiment was seen as changing very quickly, based on issues such as overseas stock exchange fluctuations, or the European debt crisis. As one developer said, "The biggest risk is are we going to sell it and how quickly are we going to sell it, and are we going to get our money back" (SD2, Small Developer)

However, they felt that the entire market was price-pointed, and buyers wanted everything for the cheapest possible price. A number of respondents commented that purchasers were not prepared to pay a premium for anything; and they would even forego previously higher priced properties (i.e. in better locations) for cheaper priced land.

\subsubsection{Green Demand}

The GFC, the currently depressed market, and difficulties in obtaining bank finance, have complex and interrelated implications for climate change adaptation. The general consensus from this study was that financial and economic issues were more important and posed a greater risk to consumers than climate change. This differed significantly between residential and commercial developers, with a marked lack of demand for sustainable (or "green") residential properties.

Prior to the GFC, some developers marketed their products as sustainable to gain a competitive advantage over their competitors. Since the GFC, the concern about sustainability and climate change had been superseded by concern about the economy. A few developers continued to construct sustainable residential projects, but the majority did not provide additional sustainability features unless required to do so under the regulations or BCA, or unless they gained a market advantage. 
In general, developers felt that those who were still building additional sustainability features were doing so out of personal conviction. For example, one consultant said, "those who are still doing it...because they actually do care about it. Most are only focussed on money"; and "at the end of the day, it comes down to cost" (CS9, Consultant).

Some developers continued to market themselves as "greener", but even these reported little public demand for sustainable products, at least in the residential sector. Given the lack of market demand, developers were largely unwilling to add sustainable features. Thus, the uncertainty of the economy and the market largely stopped developers from introducing any sustainable features other than required under the BCA or other regulation.

Few push factors were observed for more sustainable buildings in the residential market. This lack of demand was widely reported, with virtually no demographic group prepared to pay extra for sustainable properties.

"The elements of choice are not there. You are flat out making anything work economically and those sorts of things will appeal to some. People which have significant cash to spend, but there are not too many people in that space. It will change. It has to change." (CS6, Consultant).

In general, residential customers only chose to add sustainable features to their houses if the final cost was no different than the same property without them. Many purchasers had the capacity to pay for extra features, such as solar panels, but were unwilling to do so. Moreover, it was thought that customers were not willing to pay money upfront for sustainable features, even if they could save money in the long term. Further, they also wanted tangible features, such as larger rooms, rather than intangibles, such as higher cyclone ratings or lower energy costs. Investors (who bought property to hold and rent) were more likely to pay for sustainable features than were owner-occupiers.

Participants reported that most residential buyers did not care about sustainability and star ratings unless they could directly quantify any savings. For example, in extreme climates, such as Canberra, the star rating of houses was used by real estate agents as a marketing too ${ }^{13}$. However, in the milder climate of SEQ, there were few temperature extremes for which residential buildings needed to adapt. As a result, most adaptation or mitigation measures were seen as "nice to haves", but were also the first to be cut in economically stressful times.

"That adaptation thing is not really at the forefront of developer's minds. Especially with the current impacts of the GFC and that, we have much more important things, in the short term, to worry about things that may happen in 90 years' time, or over a period of time" (MD3, Medium Developer).

The lack of demand for innovative development was also blamed on the banks. Bankers stated that they were more likely to fund similar products to previous successful developments. They were unwilling to risk funding very innovative developments unless a developer could demonstrate a high proportion of pre-sales, and provide sufficient equity as security. As banks required a minimum of $75 \%$ presales, and the market was generally unwilling to pay extra for anything that was too different, developers generally considered very innovative projects as unfeasible, and highly risky, without sufficient returns.

\footnotetext{
${ }^{13}$ Energy Efficiency ratings are included in the majority of www.realestate.com.au listings for Canberra; i.e. "Eco Friendly Features = EER (Energy Efficiency Rating):Low (1.0)"
} 
This issue was also related to timing. Most banks wanted to fund developments for no longer than two years, but some funders, such as superannuation funds looked to the longer term, and were prepared to fund more sustainable developments. However, few superannuation funds currently invested in residential development, as they felt it was too risky, with too many restrictions imposed by government.

On the other hand, the commercial sector demanded buildings to have minimum sustainability ratings, such as Green Star and NABERS. Developers of commercial buildings stated that if they had sustainability ratings, they would attract "better" tenants, such as government agencies. In turn, the tenants demanded minimum certification ratings (i.e. 5 Star) of buildings. One banking representative said that the "6 Star" rating of their head office building was "the major factor in <us> moving there" (FI2, Financier). In addition, shareholders could pressure developers and corporate tenants to introduce sustainability features.

Certification was also seen as valuable because the buildings had lower operating and maintenance costs. This was very important, because the developers of these buildings generally did not sell them, but kept them for longer periods. In addition, tenants in these buildings had proven increases in productivity because of better work environments, with less sick days, and cheaper operations.

The major reason for the dichotomy between residential and commercial developers was that the latter were apparently responding to different market expectations. The commercial sector was viewed as more sophisticated and more concerned over the long term life-cycle cost of buildings. This more sophisticated market expected certain aspects and, unlike "mum and dad", were willing to pay for it. The commercial sector also reported some innovative features, such as green leases to manage carbon footprints and passive solar heating and cooling.

Respondents reported an increased demand for sustainable features by the retirement sector, but driven more by economics than a desire for sustainability, as this market demanded features such as solar panels and rainwater tanks to minimise long term costs, especially from energy price increases. This is potentially a significant driver for adaptation, as economically vulnerable sectors, such as the retirement sector, can be severely impacted by extreme climate events and price increases.

However, commercial developers were actively producing more sustainable products, and competed with one another over star ratings. This was driven by tenant demand, as well as government subsidies for certified buildings. Another major reason for this was that commercial developers tended to build and hold for the long term, rather than sell in the short term. Thus, reducing operational and maintenance costs of buildings was a major benefit of introducing more sustainable features. Despite this, banks were not prepared to fund the extra cost to build Green Star rated buildings unless a developer covered the cost with increased equity.

In conclusion, most residential developers were not building sustainable products, because of the conservatism of the banks, and lack of market demand. In addition, the economic climate overwhelmingly took precedence over sustainability.

\subsubsection{Development Finance and Climate Risk}

The finance sector is exposed to new climate change-related risks via its investment and lending activity across the economy, any part of which may be affected by:

- Physical risk - the direct impacts of increasing weather volatility and changing climatic conditions;

- Regulatory risk - the impact of government intervention at the global, regional, national or local level via legislation and market mechanisms specifically; and 
- Market and reputational risk—shifting consumer expectations and the ability of business to respond.

These risks can all impact investment value or future earnings. Financial institutions are required to understand these risks within their customer and investment base, model the specific effects on key sectors such as construction and tourism, and be prepared both for shifting industry dynamics which affect the market value of assets and unpredictable impacts on global markets (Westpac, 2009, p.7).

How climate change will influence the degree of risk of different projects, and thus the principle source of finance, is a matter of a case by case analysis. It is yet to be clear how lending institutions are responding or intending to respond to climate change and how this may influence their lending arrangements. Some banks around the world have started to integrate climate change related policies into their operational activities. For example, the European Investment Bank (EIB) has stated that, "the fight against climate change" is the Bank's priority ((EIB), 2012). Accordingly, climate change considerations are now, "systematically included in all EIB project appraisals to make the Bank's lending portfolio across all sectors more climate-friendly" (EIB 2012).

In this study, respondents generally associated risk with the potential for the loss of money or gain in profit. The industry responded well to risk if they could see the dollar value of an impact or adaptation, rather than hypothetical future problems.
"A lot of the risks have already been identified by these organisations or government and it is just putting a different lens on it, and saying what would be the impact if this were increased in duration or intensity, and what would be the implications of that and what would be the implications and cost of implementing adaptation measures which cope with the full impact rather than the current impact. Look at the cost benefit of the adaptations. I think that is the way that you sell it into business" (SC1, Sustainability Consultant).

Moreover, climate change, in concert with the organisational environment of some financial institutions, could exacerbate developer's existing lending issues. For example, insurance companies and superannuation funds tend to be very cautious about lending, particularly if they factor in increased risks from climate change. Thus, they may impose even tighter controls over the entire duration of the project-thereby diminishing the developer's autonomy (Ratcliffe et al. 2009).

Further, major banks generally funded short term projects, so sustainability was not a major deciding factor. However, some interest in adaptive development came from equity providers and superannuation funds. These had a longer term approach, and saw the approach to infrastructure and sustainability as related issues, so viewed adaption as a risk management strategy.

\subsection{Conclusions}

The most serious risks faced by property developers are financial and market risk (the risk of not selling or slow selling, of development products). These risks are, of course, correlated; developers have a limited time in which to repay bank loans, and if their development products do not sell, then they can incur serious costs, such as higher interest payments. Therefore, the way in which development is financed influences what developers are capable of; for example, how they manage and balance their various risks, the timing and scale of projects, the types and range of projects, and their potential political influence.

How development is funded is a significant factor which affects the developer's capacity to adapt to climate change. The development finance sector determines who can access private-sector funds and in what manner (under what conditions), thereby

The capacities of private developers in urban climate change adaptation 81 
ultimately influencing who can develop. Development financiers may also see climate change as a risk to their business reputation and may also have ethical or moral reasons for considering adaptation to climate change in their lending decisions.

The GFC had a major impact on the property development industry. Many developers went bankrupt, or exited the industry. This was partly due to financiers. Prior to the GFC, obtaining finance for property development was relatively easy, and many inexperienced players entered the market. During the property boom, property development was a lower risk activity; because of high demand and sustained capital growth, it was fairly simple to make a profit.

However, during and after the GFC, the property development sector suffered significantly, becoming a very high risk activity. Some lenders were seriously impacted by the GFC, with many no longer funding property development. All lenders had tightened requirements for property development, so obtaining finance is significantly more difficult. Bankers now require a higher proportion of pre-sales (in some cases, $100 \%$ ), lower LVR, higher proportion of security, and increased due diligence studies.

Banks still provide the bulk of finance to most property development firms. However, since the GFC, a larger proportion of finance is obtained from high net worth individuals, other business activities, and even international investors. Some larger companies financed their residential development activity from rental income from their commercial properties.

Participants reported very little demand for sustainable properties from the residential market. This was true across the board, from CBD to suburban; from high income waterfront to affordable units. Some demand for sustainable features, such as solar panels, was evident in the retirement living sector, but this was driven by economics and not by concern for the environment. Generally, the residential market valued immediate, tangible benefits (such as cost reductions or larger rooms) over long term, intangible benefits (such as savings on energy bills over a longer period).

However, in the commercial sector, there was a strong demand for sustainable buildings, with developers often competing with one another over Green Star or NABERS ratings. This was largely driven by the developers, who tended not to sell commercial buildings, but lease them; so saving on long term operation and maintenance costs was beneficial. Further, this was also driven by tenant demand; developers sought quality tenants, such as government agencies and large corporations, and these demanded certified or rated buildings. Finally, some commercial sustainability initiatives were driven because of government subsidies.

Climate change was not seen as a major factor in development finance or market demand. Banks did not consider climate change as a risk, particularly given the short period (less than two years) over which they generally financed development. Bankers also assumed that the consultants and regulators would have dealt with any climate change risk in the development approval stage.

Finally, developers, consultants and bankers considered that any climate change risk to the site, infrastructure or the buildings, would have been covered by the builder's insurance policies. 


\section{THE ADAPTIVE CAPACITY OF THE PRIVATE URBAN DEVELOPMENT SECTOR}

\subsection{Introduction}

"The biggest climate change challenge is the inability of the human system to respond in time to the challenge" (MD1, Medium Developer).

The primary focus of this study was to evaluate the institutional capacity of the property development sector in adapting to climate change. Limited current research exists on the adaptive capacity of the property development sector in the context of climate change (Hales, 2010; Taylor et al., 2012)). The future impacts of climate change are likely to become more intense and potentially destructive (CSIRO, 2011).

Global efforts to curb the amount of GHG emissions are underway, but the current scientific consensus is that a $2^{\circ} \mathrm{C}$ warming is unavoidable (New, Liverman, Schroder, \& Anderson, 2011). This is because $\mathrm{CO}_{2}$ and other $\mathrm{GHG}$ pollutants cannot be removed once they have reached the atmosphere and are "trapped" there for many decades. Because of this, it is more likely, that by the end of the 21st century, average global temperatures will increase by approximately $4^{\circ} \mathrm{C}$ (Anderson \& Bows, 2011; Meinshausen et al., 2009; Sanderson et al., 2011; Mark Stafford Smith, Horrocks, Harvey, \& Clive, 2011) (World Bank, 2012b, p. 16).

Human society (comprising governments, firms and individuals) has two major options to respond to climate change: it can choose mitigation (implementing measures, such as carbon pricing) to reduce GHG emissions; or adaptation (changing behaviour or ways of doing things to lessen the impacts when they occur) (Smit et al., 2000).

Mitigation attempts to limit global climate change by reducing total emissions or concentration of GHGs. It also includes efforts to enhance GHG sinks; for example, by planting trees or investing in technology such as Carbon Capture and Storage (CCS). Mitigation can also be approached through changes to land use planning policies, particularly those associated with urban density, transport provision and energy (Gleeson \& Spiller, 2010; Melia \& Barton, 2011). However, current mitigation efforts have not reduced $\mathrm{CO}^{2}$ emission levels. Therefore, the world should prepare to live with the impacts associated with a dramatic increase in heatwaves, intense droughts, major floods, and other severe weather events (World Bank, 2012b, p. 16). In other words, the world needs to engage in efforts to adapt to climate change.

\subsection{Developers Response to Climate Change}

Recent research into the attitudes of Australian developers has indicated that most do not perceive climate change as posing a major threat to their business (Taylor et al., 2012). Climate change is seen as less important to the property development industry than other priority issues, particularly regulation and the economy. Property developers tend to view secondary risks (such as changed or more onerous regulations) as posing more of a threat than direct climate impacts (Taylor et al., 2012).

Developers also respond to risk in different ways, depending on factors such as the size of the firm; perceived probability of the threat; personal factors; professional background; management expertise; financial capacity; and degree of innovation (Hales, 2010). Moreover, exposure to risk is also dependent on the type, location, and timing of development-and the developer's ongoing responsibility (if any) post development (Taylor et al., 2012). For example, development projects are usually short-term; and risks, particularly from direct climate hazards, generally fall to the owners and tenants, rather than the developer (Taylor et al., 2012). 
It is important to understand the drivers and barriers to climate change adaptation; some developers may be unable to adapt due to external circumstances, such as insufficient financial resources, or the lack of suitable and affordable land. Drivers of adaptation include; industry self-regulation, leadership and brand, and strong economic growth. Barriers to adaptation include; lack of consumer demand, financial uncertainty, and confusion in the climate policy environment (Taylor et al., 2012).

Developers can also implement practices to directly mitigate climate change and lower the total carbon footprint of their products. Such practices can be implemented throughout the phases of the development process, from appropriate design choices (materials, aspect, form, structure, etc.) to operating in the most energy efficient manner. Developers can even influence (to some degree) the level of emissions produced during the operational and decommissioning phases of their development.

If developers can respond rapidly to changing climate (and market) conditions, they will be better able to gain competitive advantage (and thus profit) over other developers. Thus, adapting to climate change can also be an opportunity for proactive developers (Bryson \& Lombardi, 2009; Hales, 2010).

\subsubsection{Adaptive Measures}

A huge variety of adaptive measures exist, some of which are relevant only to a specific area or system and others on a nationwide or global scale. Further, many adaptive measures are not specific responses to climate change, but can include other options, such as impact assessment, risk and disaster management, environmental management and environmentally sustainable development (Smit \& Pilifosova, 2003).

A range of tools and options are available to adapt to a particular climate change related impact. For example, a developer can choose to use technological advancements as a preventative measure or alternatively, market based mechanisms. Nor are these choices mutually exclusive, but can include a number of options: the subject of adaptation (who or what adapts); the object of adaptation (to what they are adapting); and how they adapt (Bosello et al., 2009; Smit \& Pilifosova, 2003).

Adaptation may also differ in its purpose (spontaneous vs. planned); its timing (proactive vs. reactive); its temporal and spatial scope (short term vs. long term; local vs. regional;) and its adapting agent (individual vs. collective; private vs. public) (Smit, Burton, Klein, \& Street, 1999; Smit \& Pilifosova, 2003). Further, adaptive measures can be differentiated into hard adaptation (i.e. technical and structural measures); or soft adaptation (i.e. regulation, improving research or using natural ecology, such as revegetation) (Sovacool, 2011).

A useful classification of adaptation response measures is offered by Burton (1996), and summarised in Table 6.

84 The capacities of private developers in urban climate change adaptation 
Table 6 Examples of Adaptive Measures (adapted from Sovacool, 2011; Smit \& Pilifosova 2003 and Burton, 1996)

\begin{tabular}{|l|l|l|}
\hline Measure & Examples of Adaptive Measure & $\begin{array}{l}\text { Types of } \\
\text { Adaptation }\end{array}$ \\
\hline Share the loss & $\begin{array}{l}\text { Purchase insurance, modifying contractual } \\
\text { agreements, etc. }\end{array}$ & $\begin{array}{l}\text { Soft } \\
\text { Planned }\end{array}$ \\
\hline Bear the loss & $\begin{array}{l}\text { Rebuild or abandon sites (could result in stranded } \\
\text { assets) }\end{array}$ & $\begin{array}{l}\text { Hard or Soft } \\
\text { Spontaneous }\end{array}$ \\
\hline $\begin{array}{l}\text { Modify the } \\
\text { events }\end{array}$ & Addressing flooding by modifying building designs & $\begin{array}{l}\text { Hard } \\
\text { Planned }\end{array}$ \\
\hline $\begin{array}{l}\text { Prevent the } \\
\text { effects }\end{array}$ & $\begin{array}{l}\text { Modify structures to withstand severe weather } \\
\text { events; structural/technological, legislative, } \\
\text { regulatory, financial, institutional, administrative, } \\
\text { and market-based mechanisms }\end{array}$ & $\begin{array}{l}\text { Hard or Soft } \\
\text { Planned or } \\
\text { Spontaneous }\end{array}$ \\
\hline Change use & Convert site use from development to greenspace & Soft, Planned \\
\hline $\begin{array}{l}\text { Change } \\
\text { location }\end{array}$ & $\begin{array}{l}\text { Choose alternate sites on which to develop, or } \\
\text { planned retreat. }\end{array}$ & $\begin{array}{l}\text { Hard or Soft } \\
\text { Planned }\end{array}$ \\
\hline Research & Improved research on new or modified adaptations. & Soft, Planned \\
\hline
\end{tabular}

In practice, adaptations include a combination of these measures. For example, coastal adaptation measures may include policies for planned retreat (changing use and/or location), and compensation for affected landholders (sharing and bearing the loss). In addition, such policies generally include other measures such as research and education campaigns.

This study found that most survey respondents (42\%) believed that their firm was incorporating climate change adaptation into decision making or current operations (Table 7). The majority of those who answered yes to this question were consultants, and one small and two medium-sized developers. Interestingly, no large developers answered yes to this question.

Table 7 Does your firm incorporate adaptation to future climate change into decision making or current operations?

\begin{tabular}{l|llllll}
\hline & \multicolumn{9}{|l}{ Category of Respondent } \\
& Small & Medium & Large & Consultant & $\%$ & Count \\
Answer Options & Developer & Developer & Developer & & & \\
Yes & 1 & 2 & 0 & 10 & $41.9 \%$ & 13 \\
No & 1 & 1 & 3 & 5 & $32.3 \%$ & 10 \\
Maybe & 3 & 2 & 1 & 1 & $22.6 \%$ & 7 \\
Don't know & 0 & 0 & 0 & 1 & $3.2 \%$ & 1 \\
\hline
\end{tabular}

The most common adaptive measure was changing site location (81.3\%); then climate resilient infrastructure (50\% - preventing and modifying the effects; and long term planning (50\%-preventing the effects (Figure 17). Although these said no the previous question, one large developer answered site location for this question. Of note, the majority of measures were selected by consultants and small developers rather than by medium and large developers. 
Three respondents also added additional comments: "we invest in continuing professional development courses for our staff"; "ongoing professional development" and "infrastructure design".

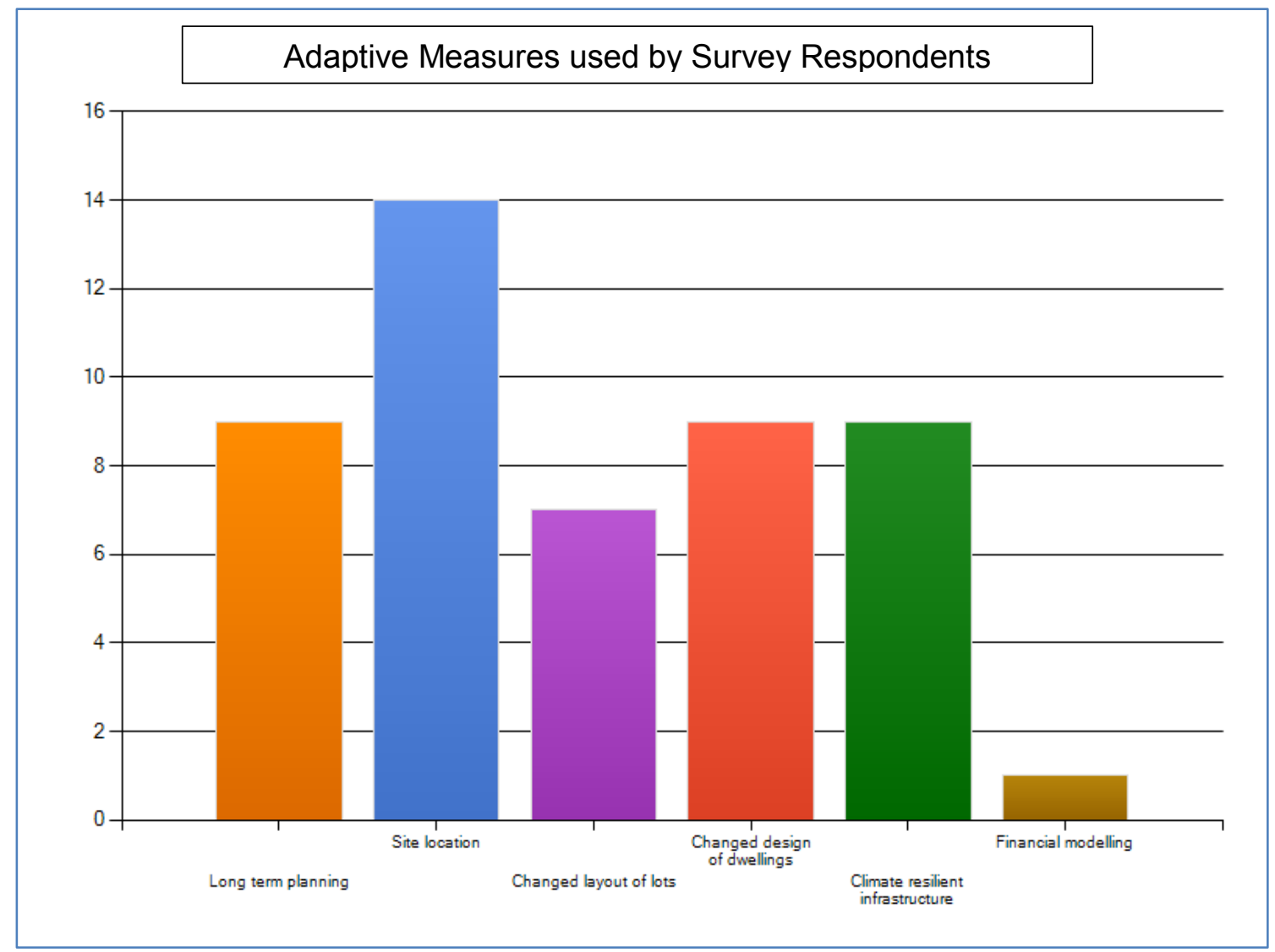

Figure 16 Adaptive Measures (Survey Respondents)

Most interview and focus group participants believed that the direct effects of climate change did not justify any adaptive response, as they felt these were not relevant in the timescales in which they developed. Many felt that responding to site risks (including severe weather impacts, such as flooding) as part of normal development risk, for which strategies were already in place. A small number said that dealing with risk in the present (i.e. of climate change) made the future less uncertain. However, the majority considered that any responses would be reactive, and they would respond to climate events when and if they occurred.

For example, a consultant said: "There are those responses that are reactive to climate change and that is, say minimum floor levels for flooding or storm surge, and they can be (not always) but they can be clear cut, they say this is what we do, and are quite easy to understand. Then there are those proactive things, that hopefully prevent climate change in the future, and...those sort of things that it is very difficult to put specific solutions on because there are so many different possibilities... and they are the really tough things to deal with" (CS4, Consultant).

Most participants apparently preferred to focus on preventing climate change impacts, prevention in this context, did not mean that they were seeking an anticipatory reaction, but rather reactive solutions to problems as, and if they occurred. The preferred solution, almost unanimously, was through practical, structural and technical measures (preventing the effects). Somewhat fewer mentioned legislative, market and financial

86 The capacities of private developers in urban climate change adaptation 
responses. However, it is important to acknowledge the financial and market environment generally dictate almost all development activities, thus, these factors strongly influence the choice of adaptive approach.

Other adaptation measures mentioned by developers included; changing location, managing social impacts, and improving education. Given that most responses could be categorised under "preventing the effects"; the following sections detail research findings under the headings, structural/technological measures; legislative measures; regulatory, financial, institutional and administrative measures, and market based mechanisms.

\section{Structural and Technical Measures}

Structural or technical solutions are the commonest types of adaptive measures used. These hard adaptive measures include; changing building codes to elevate buildings, building hard structures such as sea-walls, or soft engineering solutions. For example, Brisbane City Council raised the height limit for buildings affected by the 2010/2011 floods. Hard engineering structures, such as sea walls and levees, are expensive to build and maintain, and can have "downstream" impacts on the environment and infrastructure. Nonetheless, these are commonly used, especially in high value areas. "Soft engineering", such as beach nourishment is popular, but expensive to maintain, and can impact the environment, by changing current patterns (Vasey-Ellis, 2011).

In this study, the majority of developers and consultants felt that most direct climate change risks could be managed with hard measures such as engineering solutions and technology. This was no surprise, as planning for severe weather events, such as floods, is a normal part of development risk assessment. Further, many developers and consultants are engineers or builders, and these are practical professions.
"You could do capacity building exercises for groups focusing on practical arrangements. Because that is more engaging for those people, they probably aren't interested in being told about climate change adaptation, but they are intrigued as to how you can make these changes to the water system or the circulation system or all sorts of practical things" (CS5, Consultant).

Respondents felt that contractors (particularly engineers) had a good understanding of risk and how to design for it. However, the market played a role to what extent a developer was prepared to make any changes, particularly their competitive strategies. Some uncertainty existed about choosing to implement adaptive measures; i.e. at what adaptation point should a developer "draw the line" so as not to price a product above competitors' products.

A number of respondents suggested simple, yet practical responses to an increased risk of extreme weather incidents. For example, a consultant said, "for the actual building fabric, it is looking at the sizing and type of air-conditioning systems; the actual colouring and material used in roof design" (SC1, Sustainability Consultant). Another respondent, an architect, said, "sometimes simple engineering, like using a full brick instead of a half brick saves labour...and time and money... and raises the wall higher than the required level above a flood" (AR1, Architect). Others suggested that the easiest adaptive measures were to make houses as sustainable and energy efficient as possible; and to minimise the costs of building the development in the first place. It was considered extremely important to embed adaptive measures into buildings as early as possible; if not, they would be taken out by builders and contractors. 
One interesting finding was that single weather events could apparently drive major changes to building construction. A consultant mentioned that the result of a "tornado" that hit Brisbane in the early 1980s was a rapid change in the type of houses constructed in Brisbane. Others mentioned that the 2010/2011 floods were driving a new form of construction in flood prone areas. These adaptations included higher floor levels, sacrificial space below or moving infrastructure to higher floors.

Of note, a developer stated that equipment such as air-conditioning plants and swimming pools had a finite life. If they were going to be destroyed in a flood every 20 years or so, by then, they likely required replacing, so was it cost effective to move them? Moreover, if they were damaged by a flood, then the insurers would pay for their replacement.

Most felt that climate change would have a similar effect to that of single natural disasters, and would change the way buildings are constructed and used. They felt that the BCA and other regulations would be changed to cover sea level rise, flooding and other climate events. Moreover, some developers were already incorporating climate change risks into development, by building to 1:200 flood levels, or filling low-lying properties. They thought it was a good idea to implement passive adaptation now, such as incorporating provision for sea level rise.

A general consensus was that adapting new development to climate change could be achieved relatively simply; for example, by changing engineering parameters, such as adding new fill or building more robustly. If climate change just meant more frequent occurrence of climate events, then it would be easy to design for this. However, it was also essential that regulation changed in concert, as developers often built only to the minimum required by legislation and the BCA.

Many respondents felt that existing, not future, developments were the most threatened by climate change, and were difficult and expensive to retrofit. Another issue was providing (or maintaining) flood free access to developments. No matter how resilient a development, if access roads were flooded, it was still vulnerable to climate events.

\section{Legislative and Regulatory}

Legislative and regulatory measures are generally considered as soft adaptation, and include; the prevention and relocation of development, designation of additional protected land, the creation of wetland and setback buffers, and prevention of unsustainable land use (Vasey-Ellis, 2011). Preventing development in greenfield areas is relatively simple, and can be easily achieved through planning regulations. However, preventing development in areas where development has already been approved is much more difficult, and may result in litigation against the LGA. Relocating development is even more fraught, but may be possible in low density areas. Relocation almost always incorporates compensation (usually financial).

Designating protected land, creating buffers and revegetating vulnerable areas, can all be important adaptive policy responses. However, while protected areas are more "natural" and have little upkeep and provide environmental values; they have to be managed, usually by LGAs. Further, preventing development does not protect such land from climate change hazards. Preventing unsustainable land use can be achieved through rezoning and overlay codes, but likewise, this can negatively impact some communities, particularly lower socio-economic sectors.

In this study, respondents rarely mentioned options such as preventing or relocating development, or the other measures listed above. This is likely because these measures are preliminary to most development occurring, and form part of the process of devising LGA Planning Scheme or other regulatory zones. Most developers and consultants developed in areas that already had planning approvals in accordance with

88 The capacities of private developers in urban climate change adaptation 
existing LGA zones, because of bank lending requirements and the current risk averse nature of the industry.

With regard to regulation and legislation in general, many respondents felt that the development industry was not leading the way regarding climate change, and the industry was largely reactive to changes imposed by government. Most developers would only take climate change into account when they were required to do so by law. If they took additional adaptive measures not required by law, then they ran the risk of overpricing their products, and market failure.

Therefore, most thought that regulation would be a major driver of adaptive measures; for example, LGAs would communicate any changes to flood levels, and developers would adapt, by building higher or filling lots. It was also assumed that the BCA and LGA design codes were constantly updated in response to forecast climate risks.

On the other hand, some thought that adapting to climate change was not driven by regulation, but by industry innovation and standards, and client demands. For example, clients demanded adaptive measures in commercial buildings after the Brisbane floods, because they did not want to be out of business for any extended period of time. However, these responses referred only to commercial and not residential development.

Others (mostly consultants) felt that legislation was a blunt instrument, and alone, was inadequate to adapt to the challenge of climate change. Key to an effective regulatory regime was to establish benchmarks, and measure performance against these to reach the benefits, which would then be regulated.

However, as an engineer asked, "how much science is there in figures, for example, a 10\% increase in something, like rainfall intensity, is it just a figure pulled out of the air, to seem like governments are doing something... anything?" (CS8, Consultant).

\section{Market based}

Market-based measures are also forms of soft adaptation, and are largely spontaneous. Market-based adaptive measures include letting developers accept full development risk, or relying on private insurance for vulnerable properties and areas. For example, accepting full risks and responsibility for compensation is effectively used in other industries, such as road construction (i.e. paying an environmental bond). However, any penalties imposed have to be sufficiently high to act as a deterrent (i.e. the cost of remediating the mistake is significantly higher than the fine).

Further, if the sole responsibility for protecting vulnerable areas is left to market forces, this may well lead to effective outcomes, but it may also lead to owners of high value properties lobbying governments for extra protection for their properties; for example, to build hard defences, such as seawalls, or even legislative change.

Some commented on the negative market implications of early adaptation; saying it would disadvantage them in comparison to their competitors. This was partly due to the current post GFC economic climate, which was generally a barrier to innovation.

Further, banks would not lend money for untried products, unless a significant amount of pre-sales (at times, 100\%) was achieved. In addition, the market was not prepared to pay for innovation, especially if this was more expensive than the standard product.

Consultants felt that adaptive measures (or sustainability features) were regularly included in large master planned developments, but were often discarded in smaller developments, as "the nice to haves are cut first" (SG1, State Government). Therefore, 
it was important that such features embedded into contracts, otherwise the contractors or tradespeople could persuade the client that the measures were too expensive.

"The economic analysis of adapting now for something that might not eventuate in 90 years has not been done. It might be better deal with things when they occur; then the technology will be better, and a mitigation strategy can be implemented. The solution is probably somewhere between adapting now and mitigating when it occurs" (CS8, Consultant).

\section{Education, Information, Communication and Awareness}

Access to education and sources of knowledge are soft adaptations, and essential for adaptive capacity; leading to an increased ability to understand and prepare for the consequences of climate change. An increased knowledge and awareness of climate change can influence attitude, and thus behaviour (Trumbo \& O'Keefe, 2005). However, communication of incorrect information (such as espoused by climate change deniers) can also lead to maladaptation; i.e. a developer may feel adapting is unnecessary.

"There is a need for education around adaptation to climate change, the smaller bits and pieces of development don't really accommodate that. In comprehensively developed areas, that feature of community building has been an attraction for people buying into those areas anyway, so it has a potentially added benefit" (CS7, Consultant).

A few respondents felt that there was a need for education around adaptation to climate change. However, there was a degree of cynicism around teaching current members of the industry, and it was felt that it really should be taught to the next generation, as part of school curricula. Further, some emphasised that it was essential to communicate adaptive measures to everyone within a firm, including sales people and subcontractors.

\section{Complexity of Adaptive Measures}

One finding that could have potential benefits for adaptation was the acknowledgment by some that adaptive measures should be viewed as part of a complex system. Any adaptive measures, or combination of these, were complex and interrelated. A number of respondents noted that localised or on-site adaptive measures could actually be maladaptive.

"Traditionally a lot of consultancies looked at only the physical risks, but we also look at the social impacts of those hazards. For the rail project we looked at the climate impact of sea level rise on the bridges or track work and we look at the impact of comfort for passengers on the trains, and also the protection of passengers waiting at train stations... we also look at the accessibility to the train lines. Some communities may be physically cut off or due to the extreme temperatures that we have projected for as part of the assessment, which may not be an option for people to even walk in certain weather to get to the train line. So it was looking at the social impacts as well as the physical impacts" (SC1, Sustainability Consultant); and

"We need to think more holistically. For example, the ongoing development of road infrastructure, what about rail and cycleways? We need a more robust public transport network that can also withstand climate challenges" (AR2, Architect).

90 The capacities of private developers in urban climate change adaptation 
For example, adaptation options could include; preventing development, ignoring risk, transferring risk by using insurance, and using design measures. However, all of these involve trade-offs. Measures such as filling flood prone land can displace the problem, and can affect neighbours, thus a developer may be liable for litigation.

Other examples given included: sea walls change ocean currents and impact sand transport and beach nourishment; raising levels could make access difficult; ignoring risk could have major financial disbenefits; and not allowing development could have legal and property rights issues. Moreover, some measures, such as relocating existing development would have virtually no industry or community support.

Others suggested that soft adaptations, i.e. mimicking nature, would provide the greatest resilience; for example, using natural vegetation and waterway lines in developments. Using passive design measures, and biodiversity options, such as planting native trees for increased shade, cost less than technological options, and also provided climate mitigation benefits.

"Changes in landscaping to incorporate more passive ventilation, and also the positioning of certain road networks to increase the buffer for bushfire protection. Increasing water sensitive urban design elements to improve the drainage, and to reduce demand on potable water use. A lot of the initiatives that developers have been doing for years now, but just couching it in a climate adaptation perspective" (SC1, Sustainability Consultant).

One industry representative felt "adaptation would mean a number of measures addressing each of those threats of climate change across the site" (IN1, Industry Representative). Some developers were reportedly already incorporating features that could be quickly changed in order to adapt to climate change; as well as including resilient and easily accessible public transport infrastructure.

\subsection{Adaptive Capacity}

Adaptive capacity is the adjustment of a (human or natural) system in response to the actual or anticipated impacts of climate change, by moderating harm or exploiting beneficial opportunities (Smit \& Pilifosova, 2003). Therefore, adaptive capacity goes beyond the ability to cope with the negative impacts of climate change. Rather, it includes the ability to decrease vulnerability, improve resilience, and capture the potential benefits that may be presented by climate change.

Moreover, climate risks themselves can drive adaptation to climate change: either as direct risks (i.e. flood threat to a development) or non-climatic risks (i.e. changes to regulation). In general, developers respond more to the threat of non-climatic risks, especially changes to the regulatory environment (Berkhout et al. 2006; Taylor et al. 2012). However, many developers do not view climate change risks as separate to other market risks (Berkhout et al. 2006; Taylor et al. 2012).

"Adaptation or resilience language is starting to come into the day-today language of business; it directly relates to risk management, which is integral to development" (SC2, Sustainability Consultant).

The scientific literature of adaptive capacity offers a number of methods for measuring adaptive capacity. One common approach is to empirically evaluate how a system has responded in the past to extreme weather events (e.g., storm surges, floods, etc.) and based on this, project it's response to similar stress events in the future (Engle, 2011). While this approach has been used extensively in the climate adaptation field (Adger et al., 2007; Bussey et al., 2011); its limitations have been highlighted by Engle (2011). 
According to Engle (2011) this approach to measuring adaptive capacity may fail to produce accurate results due to two main reasons:

1. Past events may not be comparable with future events. In other words, climate change may exceed what the system has experienced in the past.

2. Some changes/ responses to events occur incrementally and in conjunction with a range of processes that can influence (constrain or enhance) adaptation. These processes may include technological innovations, change of government and a consequent paradigm shift, etc.

Another method to evaluate adaptive capacity is to use a combination of factors in a range of places and scenarios. Thus, the adaptive capacity of a human system, such as a property development firm, could depend on determinants such as access to technology, information and skills, economic resources, the structure and governance of institutions and human capital (World Bank, 2012a) adapted from ((UNDP), 2012; Adger et al., 2007; Smit \& Pilifosova, 2003)UKCIP, 2003; IPCC, 2001). Moreover, adaptive capacity is not static, and may respond to any changes in one or more of these often interrelated determinants (Smit \& Wandel, 2006).

However, Engle (2011) argues that using pre-existing determinants to evaluate adaptive capacity limits the potential to reveal what factors actually affect adaptive capacity: "Measuring (adaptive capacity) aims to build theory while characterizing primarily applies theory" (Engle 2011, p. 653).

Further complicating the matter, some drivers may also be barriers, depending on circumstances or on a specific firm. For example, acquiring information about climate change may lead to a clearer understanding and assessment of the risks of climate change, and thus motivate action. On the other hand, the 'wrong' type of information about climate change may lead to scepticism and inaction (CSIRO 2011). Due to this complexity, the measures of adaptive capacity are not discussed in terms of drivers or barriers, but how each may help or hinder adaptive capacity.

This study found that the indicators of adaptive capacity could be organised under five categories, in a dynamic combination of often interrelated determinants, that respond differently, according to a range of places and scenarios (Adger et al., 2007; Smit \& Pilifosova, 2003; Smit \& Wandel, 2006)IPCC,2001). The categories were as follows:

- Participant Characteristics: Developers and Consultants/Experts;

- Internal Characteristics: Attitude, Communication, Information and Skills, Community and Society, and Leadership, Legacy and Brand;

- Development Characteristics: Access to and Availability of Technology, Ability to Change Use and/or Location, Time and Scale of Development, and Type of Development.

- Economic and Market Characteristics: Economic Resources of a Firm, Economic Environment, Financiers, Insurance Industry, and Market Demand;

- Governance, Regulation and Legal Characteristics: Developer led Standards and Certification; Governance and Regulation; and Legal;

The major determinants of adaptive capacity found in this study are summarised in Table 8 (and Figure 25), and discussed below. Of note, adaptive capacity is flexible, dynamic and dependent on a number of interrelated determinants (Smit \& Wandel, 2006).

"Adaptive capacity changes all the time. You may be resilient one week but not resilient the next" (SC2, Sustainability Consultant). 
Table 8 General Measures of Adaptive Capacity (expanded from classifications of (Smit \& Pilifosova, 2003) and (Burton, 1996).

\begin{tabular}{|c|c|}
\hline Determinant Type & Examples of Adaptive Capacity \\
\hline \multicolumn{2}{|l|}{ Participants } \\
\hline $\begin{array}{l}\text { Consultants and } \\
\text { Other Experts }\end{array}$ & $\begin{array}{l}\text { Firms with a greater ability to access external (or in house) } \\
\text { sources of specialist knowledge are better able to adapt. }\end{array}$ \\
\hline $\begin{array}{l}\text { Developers (Firm } \\
\text { Size \& Structure) }\end{array}$ & $\begin{array}{l}\text { Larger developers with a more diverse property portfolio have } \\
\text { greater flexibility and economic resources for adaptation. }\end{array}$ \\
\hline \multicolumn{2}{|c|}{ Internal Characteristics } \\
\hline Attitude & $\begin{array}{l}\text { Accepting attitudes towards the risk of climate change improve } \\
\text { adaptive capacity, whereas scepticism can be a barrier. }\end{array}$ \\
\hline $\begin{array}{l}\text { Communication, } \\
\text { Information, Skills }\end{array}$ & $\begin{array}{l}\text { The greater the information available, the greater the capacity to } \\
\text { adapt, and to learn new skills from that adaptation. }\end{array}$ \\
\hline $\begin{array}{l}\text { Community and } \\
\text { Society }\end{array}$ & $\begin{array}{l}\text { Firms that are committed to building strong communities in } \\
\text { completed developments are more likely to promote adaptation. }\end{array}$ \\
\hline $\begin{array}{l}\text { Leadership, } \\
\text { Legacy \& Brand }\end{array}$ & $\begin{array}{l}\text { Firms concerned about legacy and brand are more likely to have } \\
\text { a longer term outlook, with improved adaptive capacity. Firms } \\
\text { with a greater degree of social and business capital have a } \\
\text { greater capacity to recover from climate change impacts. }\end{array}$ \\
\hline \multicolumn{2}{|c|}{ Development Characteristics } \\
\hline $\begin{array}{l}\text { Access/ Availability } \\
\text { of Technology and } \\
\text { Innovation }\end{array}$ & $\begin{array}{l}\text { Openness and the ability to use new, innovative technology } \\
\text { improves adaptive capacity. Developments with quality } \\
\text { infrastructure (i.e. appropriate cyclone rated buildings) have a } \\
\text { greater capacity to manage the impacts of climate change. }\end{array}$ \\
\hline $\begin{array}{l}\text { Change use and/or } \\
\text { Location }\end{array}$ & $\begin{array}{l}\text { Flexible development types that can be modified for other uses, } \\
\text { or even more, are more adaptive. }\end{array}$ \\
\hline $\begin{array}{l}\text { Time \& Scale of } \\
\text { Development }\end{array}$ & $\begin{array}{l}\text { The spatial and temporal scale of a development is intrinsic to } \\
\text { adaptive capacity; longer term, larger developments are more } \\
\text { likely to take climate change risk into account. }\end{array}$ \\
\hline $\begin{array}{l}\text { Type of } \\
\text { development }\end{array}$ & $\begin{array}{l}\text { Commercial and retail developers are introducing adaptive } \\
\text { measures into buildings; residential developers are yet to do this } \\
\text { to any extent, mainly due to lack of demand. }\end{array}$ \\
\hline \multicolumn{2}{|c|}{ Economic and Market Characteristics } \\
\hline $\begin{array}{l}\text { Economic } \\
\text { Resources }\end{array}$ & $\begin{array}{l}\text { Higher income firms have improved adaptive capacity; they have } \\
\text { better access to information, technology and external specialists. }\end{array}$ \\
\hline $\begin{array}{l}\text { Economic } \\
\text { Environment }\end{array}$ & $\begin{array}{l}\text { Strong economic growth can promote innovation and adaptation, } \\
\text { and weak growth can hinder it }\end{array}$ \\
\hline Financiers & $\begin{array}{l}\text { Finance is vital for most developers. The conditions placed on } \\
\text { development loans can help or hinder adaptive capacity. }\end{array}$ \\
\hline Insurance Industry & $\begin{array}{l}\text { The insurance industry may drive or prevent adaptation; higher } \\
\text { premiums may motivate adaptations, or lead to underinsurance. }\end{array}$ \\
\hline Market Demand & $\begin{array}{l}\text { Developers are more likely to implement adaptive measures if the } \\
\text { market demands, and is willing to pay, for these. }\end{array}$ \\
\hline \multicolumn{2}{|c|}{ Governance, Regulation and Legal Characteristics } \\
\hline $\begin{array}{l}\text { Developer } \\
\text { Standards }\end{array}$ & $\begin{array}{l}\text { Developer led standards and certification have a major potential } \\
\text { to act as drivers of adaptation. }\end{array}$ \\
\hline $\begin{array}{l}\text { Governance and } \\
\text { Regulation }\end{array}$ & $\begin{array}{l}\text { Developers working in areas with strong institutions are better } \\
\text { able to adapt to climate change. Political and institutional } \\
\text { inefficiencies and delays hinder adaptation. }\end{array}$ \\
\hline $\begin{array}{l}\text { Legal and } \\
\text { Litigation }\end{array}$ & $\begin{array}{l}\text { Related to governance and regulation; litigation or the threat of } \\
\text { this, can promote either adaptation or maladaptation. }\end{array}$ \\
\hline
\end{tabular}

The capacities of private developers in urban climate change adaptation 93 


\section{General Measures of Adaptive Capacity (Survey Respondents)}

Most survey respondents (45.5\%) stated that their firm had incorporated climate change adaptation into decision-making (Figure 18). However, a third (30.3\%) said their firms had no adaptive plans. When splitting the dataset by respondent category, and running a one way ANOVA, there were no significant differences between questions, with the exception of, "Adapting to climate change now will have future benefits" $(F(3,30)=3.294, p=0.034)$. For this question, significant differences were noted between large and medium developers, and small developers and consultants; with larger developers more likely to rate this as important.

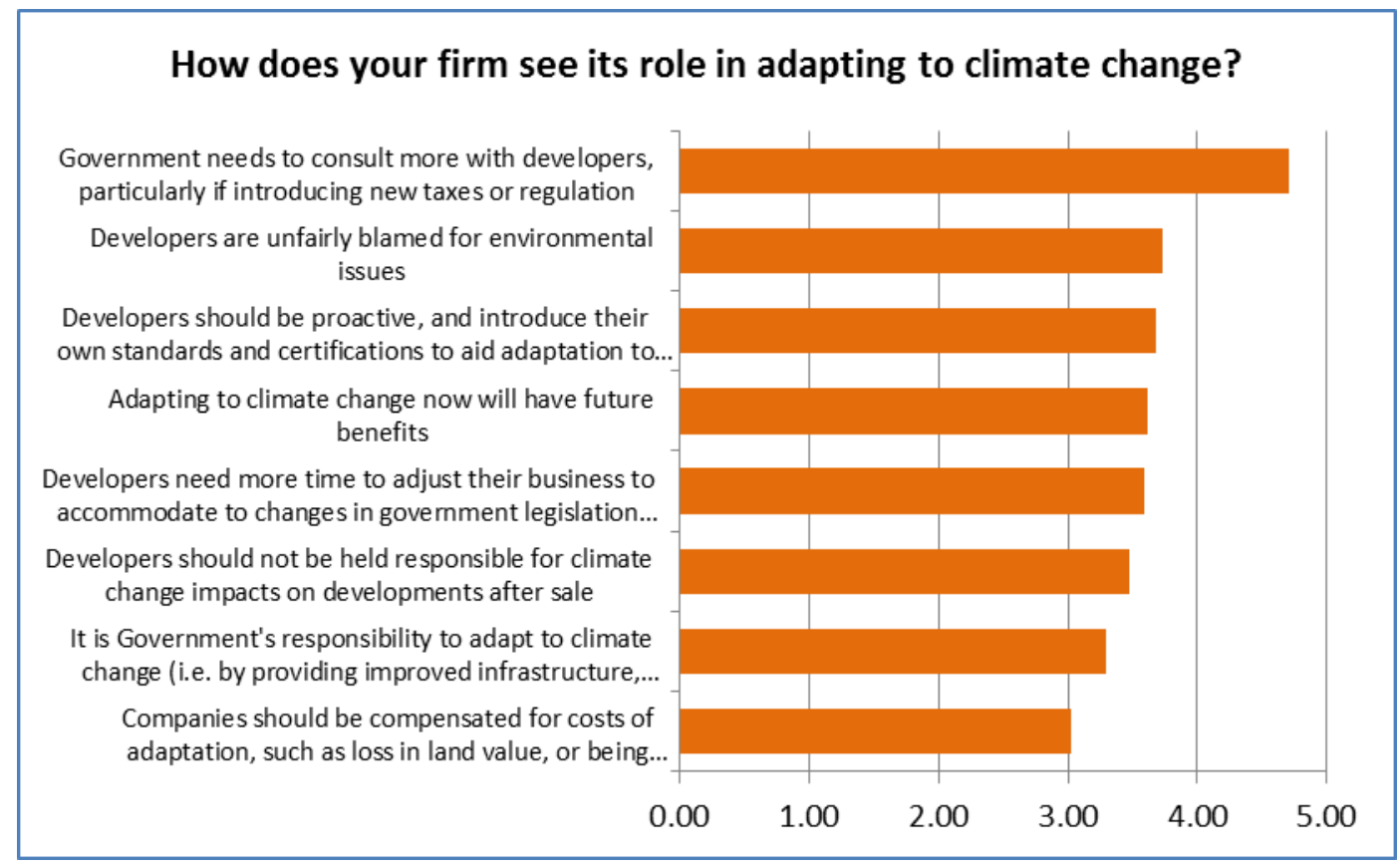

Figure 17 Survey Respondents Opinions on General Adaptability Issues

When the question was asked, "what would make your firm more likely to implement climate change adaptation measures", survey respondents rated the majority of options as 3 (neutral) to 4 (important). As indicated in Figure 19, the most important driver was regulatory (reduced DA approval time), but the following four were economic (increased profitability, increased competitive advantage, changes in market demand, and incentives or benefits). Most internal characteristics, such as ethical or moral issues, were ranked relatively low.

There was no significant difference between respondent categories in answering the questions, with the exception of legislation and regulation obligations. Most large developers felt this was more of a driver than did consultants or small developers $(F(3,30)=6.767, p=0.001)$. In general, large and medium developers considered all of these as more important drivers than did small developers or consultants. However, no respondents said that any of these were significant drivers of adaptation. Further, relatively few large developers participated in the study $(n=6)$.

94 The capacities of private developers in urban climate change adaptation 


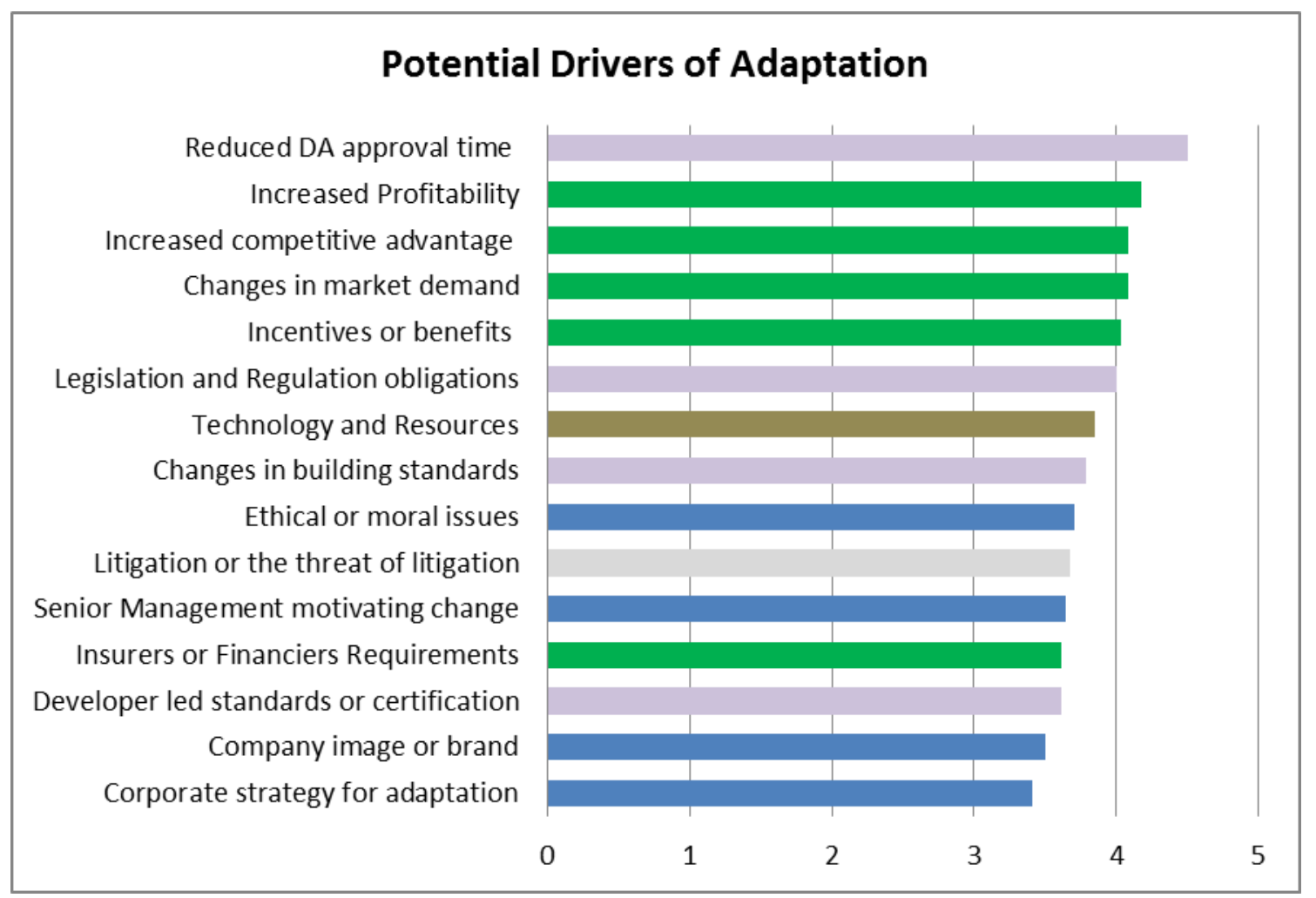

Figure 18 Potential Drivers for Adaptive Capacity (Survey Respondents)

(green = economic; lilac = regulatory; blue = internal; brown $=$ development $)$

Survey respondents ranked barriers to adaptation much higher than they did drivers of adaptation (Figure 20). The major barrier was seen as lack of market demand, then added costs and additional time needed for adaptation. For some questions, respondents answered similarly (i.e. lack of market demand and added cost), but for other questions, answers differed according to respondent type. For example, small and medium developers were sceptical about the need to adapt to climate change, whereas consultants generally agreed it was important to adapt. However, a one way ANOVA showed no significant differences between respondent categories for any questions. 


\section{Barriers to Climate Change Adaptation}

Lack of market demand

Added cost

Extra time needed

Policy and Regulatory confusion

Sceptical about necessity

Lack of access to technology

Subcontractor behaviour

Not part of Corporate Strategy

Lack of expertise

Lack of knowledge

Employee behaviour

No Shareholder / Board approval

Unconvinced of added value

Not relevant in development timelines

Uncertain of climate change risks

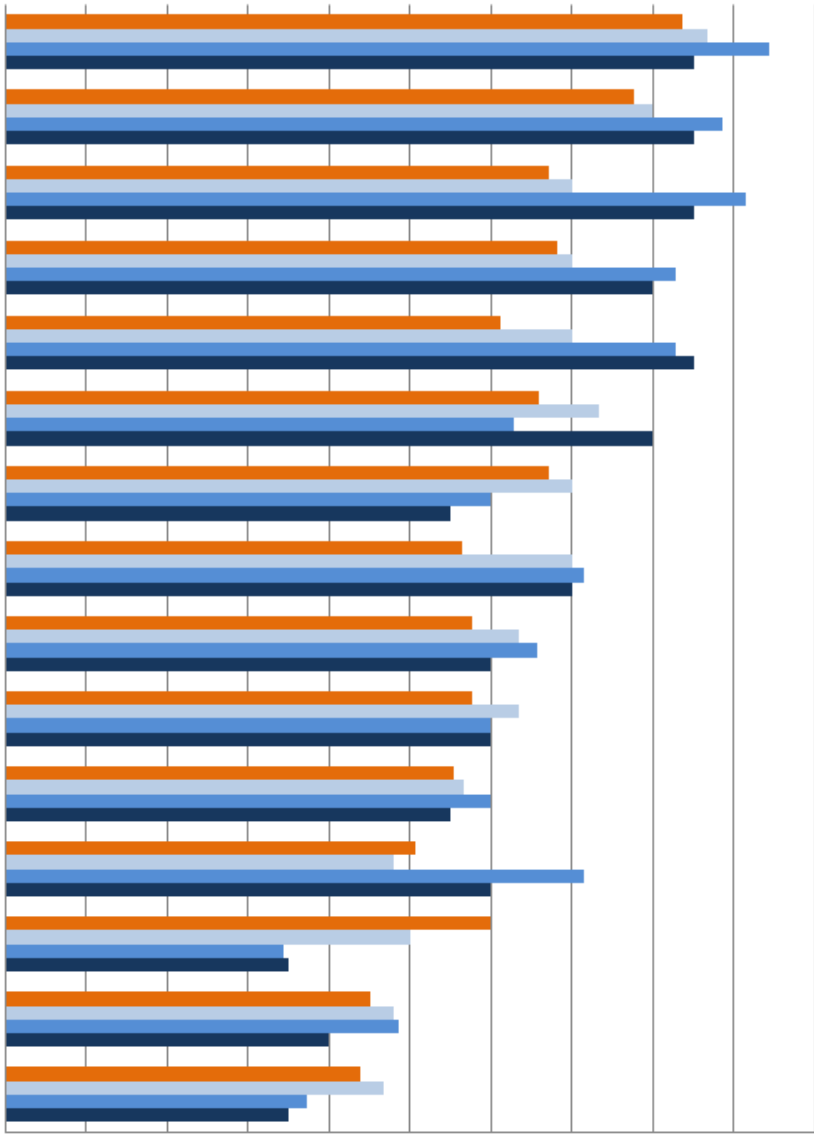

$\begin{array}{lllllllllll}0.00 & 0.50 & 1.00 & 1.50 & 2.00 & 2.50 & 3.00 & 3.50 & 4.00 & 4.50 & 5.00\end{array}$

- Consultant etc $\square$ Small $\square$ Medium $\square$ Large

Figure 19 Potential barriers to climate change adaptation (survey respondents)

\subsubsection{Participant Characteristics}

\section{Consultants and Other Experts}

The ability to source information and expertise from outside a development firm could be a significant driver of adaptation. Professional consultants specialise in many aspects of development, ranging from engineering to sustainability. Like developers, consultancies were extremely diverse, ranging from large multinational organisations to small, one-person operations.

In this study, the majority of developers used a range of consultants and subcontractors. Some aspects of development were usually outsourced to consultants, such as preparing and submitting planning and development applications. Larger developers generally had better resources to hire (or employ) professional experts, such as sustainability consultants, when necessary.

Consultants were a major source of information for survey respondents, and other participants in the research. These consultants primarily sourced their information from State Government, LGAs and the UDIA. Financiers were also heavily reliant on information provided to them by external sources, such as consultants and valuers. 
"That's why we are members of the UDIA, and got involved with EnviroDevelopment, and talk to the consultants...they keep up to date with the requirements. There are the ones who say, how about you consider this/that. Read all the magazines. Cooperative effort, deciding in design process, what you do, what you incorporate, how you meld it, then you put the budget in and see what you can afford; and then you work out what is the happy medium" (SD2, Small Developer).

Attitudes to climate change and the necessity of adaptation differed widely amongst consultants. The majority of planning consultants interviewed were, to some extent, sceptical about anthropogenic climate change. They were also extremely critical about regulation, such as the Coastal Plan. However, a smaller proportion, comprising sustainability consultants, and employees of large consultancies, were extremely knowledgeable about climate change, and climate change adaptation.

Unlike consultants, subcontractors, such as builders and other tradespeople, were viewed as extremely conservative and risk averse. These subcontractors had significant influence on building sites, and were reportedly unwilling to use innovative or new materials. In some cases, developers said the builders had persuaded the client not to use a sustainable product, because the standard product was cheaper and the tradespeople on site were more experienced in its use.

"If builders and contractors can make a saving, they will cut any adaptation initiative that they can, if they see it as a cost or if it hasn't been securely locked into a contract or consent approval. It will definitely be left off. It is very important to consider adaptation right at the early stages so it is just embedded as part of the concept plan or concept design or the master plan for a project. So that it is right there for the start. If you try and bolt it on at the end, it is not integral as part of the project. That will then be identified by the contractor or the constructor as a cost saving"(SC1, Sustainability Consultant).

\section{Firm Size and Structure}

The internal organisation and size of a development firm also influences the degree of control over the development process and the quality of the final product; the firm's capacity to tailor its product to the market and to adjust supply to changing and emerging circumstances (Coiacetto, 2007). The size of a development company can directly influence not only a developer's operational strategies, but other factors, such as exposure to risk, lending ability and type of development products. Further, increasing the institutional scale and geographic scope of operations (i.e. operating over a number of states) can give a development firm more opportunity to spread risk of either innovation or market variation. Being bigger allows developers to spread risks geographically and sectorally, and across submarkets within sectors.

In this study, respondents generally assumed that larger developers had greater adaptive capacity, because they had better internal resources, were less dependent on bank finance, and had access to specialised services, such as consultants. Smaller developers were considered to have less flexibility and more dependence on external advice and contractors.

Many developers were viewed as reactive, slow to innovate and not future focussed (except on profit). Climate change was not viewed as posing any greater risk than any other issue; and a degree of risk was expected and planned for every development. In addition, as property developers are only liable for 7 years after completing a development, they felt the short timeframes negated most future risk.

Therefore, climate change was not seen as a serious risk to most developers, unless it affected their development and hence their profits. Many stated that no major

The capacities of private developers in urban climate change adaptation 97 
adaptation initiatives were likely to occur unless major climate events happened, more frequently: "We should start to adapt when we start seeing evidence of something actually happening" (CS9, Consultant).

However, some developers, such as those specialising in the aged and retirement sector, were taking account of climate change risks, and implementing adaptive measures. This was, to some extent, driven by economic factors and this market segment wishing to reduce energy costs. Another driver in this sector was the regulators requiring measures such as backup generators in case of power failure.

\subsubsection{Internal Characteristics}

\section{Attitude}

Most Australian developers do not perceive climate change as posing a major threat to their business (Taylor et al., 2012). General opinions were that climate change (and the urgency of adaptation and mitigation) had "dropped off the radar since 2007" and had been superseded by concern over the economy (SC2, Sustainability Consultant). In general, developers only considered climate change and other sustainability aspects of development when the market demanded it.

Significant scepticism regarding the anthropogenic causation of climate change was apparent, mainly from planning consultants. The sceptical respondents often cited their own histories as evidence for not doing anything:

"We've had a few cyclones here....and generally we have come off pretty softly" (CS9, Consultant);

"We got flooded in 1974 in Brisbane but it hasn't stop me from living in a flood prone city" (CS3, Consultant); and

"I've seen a lot of climatic events, a lot of change. I've seen cyclical events. Having grown up on the beach, watched the same piece of beach for so many years...the greatest influences I see are those affected by people rather than climate" (CS9, Consultant).

For some, climate change was a matter of belief and personal experiences, and therefore, not a major risk to their business. As a small developer said, "Climate change is how you feel. Some... are concerned about it and some are not" (SD1, Small Developer). This was exacerbated by the complex nature of the issue and the diverse range of information on what actually comprises climate change. For example, some respondents considered that ozone depletion and tsunamis were impacts of climate change. Even if respondents correctly identified the impacts of climate change, they tended to associate it primarily with sea level rise, which they saw happening in the distant future; therefore not posing any serious threats.

Therefore, a major issue regarding attitude was the mistaken belief that climate change would only happen in the distant future, and thus only posed a risk to major developers with lengthy, large-scale projects. The very intangibility of climate change, coupled with the temporal disconnect between cause and effect (i.e. between increased $\mathrm{CO}^{2}$ and future climate events, or between mitigation measures and reduced $\mathrm{CO}^{2}$ ), led to some degree of apathy.

Such negative attitudes can directly influence adaptive capacity. If most developers and consultants do not view climate change as a risk, they are less likely to implement adaptive measures. As one (sceptical) consultant put it, "they are not going to spend any more money on something they don't even perceive is a real threat" (CS3, Consultant). Further, some may actively work against adaptation, by directly lobbying governments to change legislation. Arguably, this has already occurred in Queensland, and led to the suspension of the State Planning Policy 3/11: Coastal Protection (SPP). 
However, many respondents mentioned, "doing their bit for the environment"; "wanting to leave a legacy for their children" or promoting Environmentally Sustainable Development (ESD). Further, when prompted at the end of interviews, respondents emphasized that increased incidence of severe floods or cyclones (as examples) were major risks to their business. Interestingly, two consultants felt that lengthy climate events (such as droughts) were more indicative of climate change than short, catastrophic events, such as cyclones.

Therefore, many of the negative attitudes seem more due to misinformation and poor communication by policy makers, the media and even academics, rather than to a lack of concern for the risks of climate change. Improving education and communication is one way in which this can be overcome.

Attitudes on the necessity of adaptation were also related to the timing of development; those engaged in short-term development projects generally did not view climate change as a major risk.

\section{Communication, Information \& Skills}

Companies (and developers) rarely adapt spontaneously to a given issue (Berkhout et al. 2006). A necessary antecedent to adaptation is sensitisation to an issue; and this is not possible without information, which may take the form of specialised knowledge, leadership and/or prior experience (Berkhout, Hertin, \& Gann, 2006; CSIRO, 2011).

"Property developers who will survive will be <those $>$ who have deep understanding of climate change, a deep understanding of the risks that are beyond the current regulations" (SC2, Sustainability Consultant).

This study found a wide variety of climate change knowledge in the development industry; from the developers themselves, to the consultants, subcontractors and regulators. In the survey, all developers and consultants considered that they had an average to very good knowledge about climate change, with the majority stating that their knowledge was good (Figure 21). However, in the interviews and focus groups, it was obvious that many respondents did not have a good knowledge of climate change. Many considered that climate change meant only sea level rise; and others referred to tsunamis, earthquakes and ozone depletion.

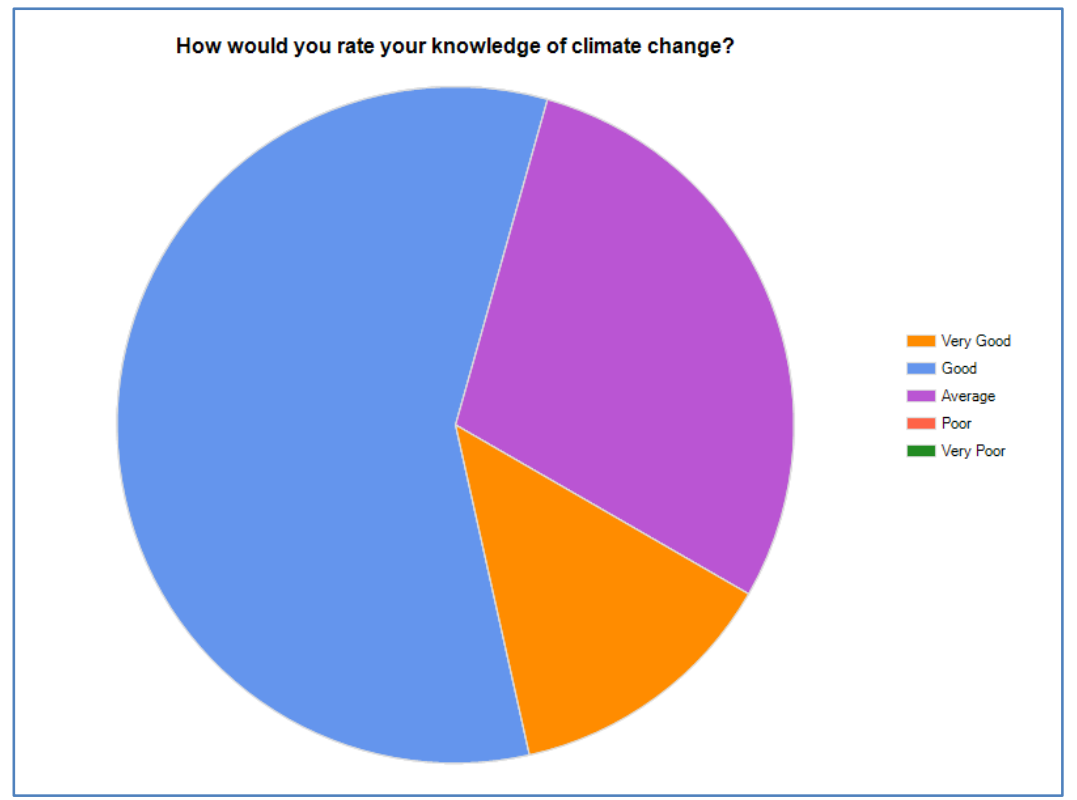

Figure 20 Survey Respondents Self Rating of Climate Change Knowledge 
Survey respondents obtained their information from a range of sources, including the UDIA, magazines, journals and the internet. In the interviews and focus groups, some respondents stated that their major source of information was consultants, who primarily sourced their information from State Government and LGAs. Others also mentioned sourcing information from the UDIA.

However, the risks of climate change were consistently miscommunicated, for the most part by the media. A large proportion of the respondents associated climate change primarily with sea level rise, possibly due to the media portrayal of melting ice caps and stranded polar bears. Others were strongly sceptical and apparently influenced by nonacademic sources, such as radio "shock jocks" and certain newspapers. Interestingly, the major point of scepticism appeared to be the anthropogenic causes of climate change, and not that the climate was changing.

Thus, most people had a limited understanding of climate change, so did not fully understand the issues and consequences of not adapting. Further, many did not understand statistics, probability, or even what Q100 meant.

However, a sustainability consultant stated that governments were equally to blame, as they often did not release full risk modelling, and instead released a more conservative model because of the political implications of "frightening the electorate". Therefore, the information flow about real risks, such as exposure to storm surge, was sometimes significantly understated. Further, even if the information were available, it was often extremely difficult and/or expensive to navigate government "silos" to obtain it.

One aspect of information that could have negative impacts on adaptive capacity was the reliance on external sources of information, predominantly from government agencies; and the assumption that this information is complete, valid and current. In addition, some respondents (i.e. financiers) assumed, possibly incorrectly, that regulators and consultants had taken account of all information regarding a site.

Given the conflicted knowledge of climate change and adaptation, an identified need for further education was identified. One way in which this could be done was to use visual tools such as Geographic Information Systems (GIS) to communicate climate change risks. However, a related issue was the knowledge and education of the educators and trainers; were these people sufficiently qualified to communicate the complex subject of climate change to developers?

Another issue was the politicisation of the term "climate change". The sustainability consultants interviewed felt that the phrases "climate change" and "global warming" were loaded terms. This was partly due to the association of climate change with radical environmentalists, who were seen as diametrically opposed to business, such as property development. Therefore, some felt it preferable to couch the issue in alternative terms. For example, many developers considered sustainability or energy efficiency as synonymous with adaptation or mitigation. In addition, communicating how it could be a financial gain would be beneficial to developers.

Finally, the information available on climate change and adaptation was complex, poorly communicated, difficult to obtain from government agencies, and often communicated in a biased manner by those with vested interests.

"I think all the solutions are there, but it is the enablers of that and how to connect it purposefully?" (LD2, Large Developer); and

"The development industry is open to being led in the right direction, but what is the right direction" (SC2, Sustainability Consultant). 


\section{Leadership, Legacy \& Brand}

One major finding of this study was that the concepts of corporate leadership, legacy and brand were strong drivers of adaptive capacity (Figure 23).

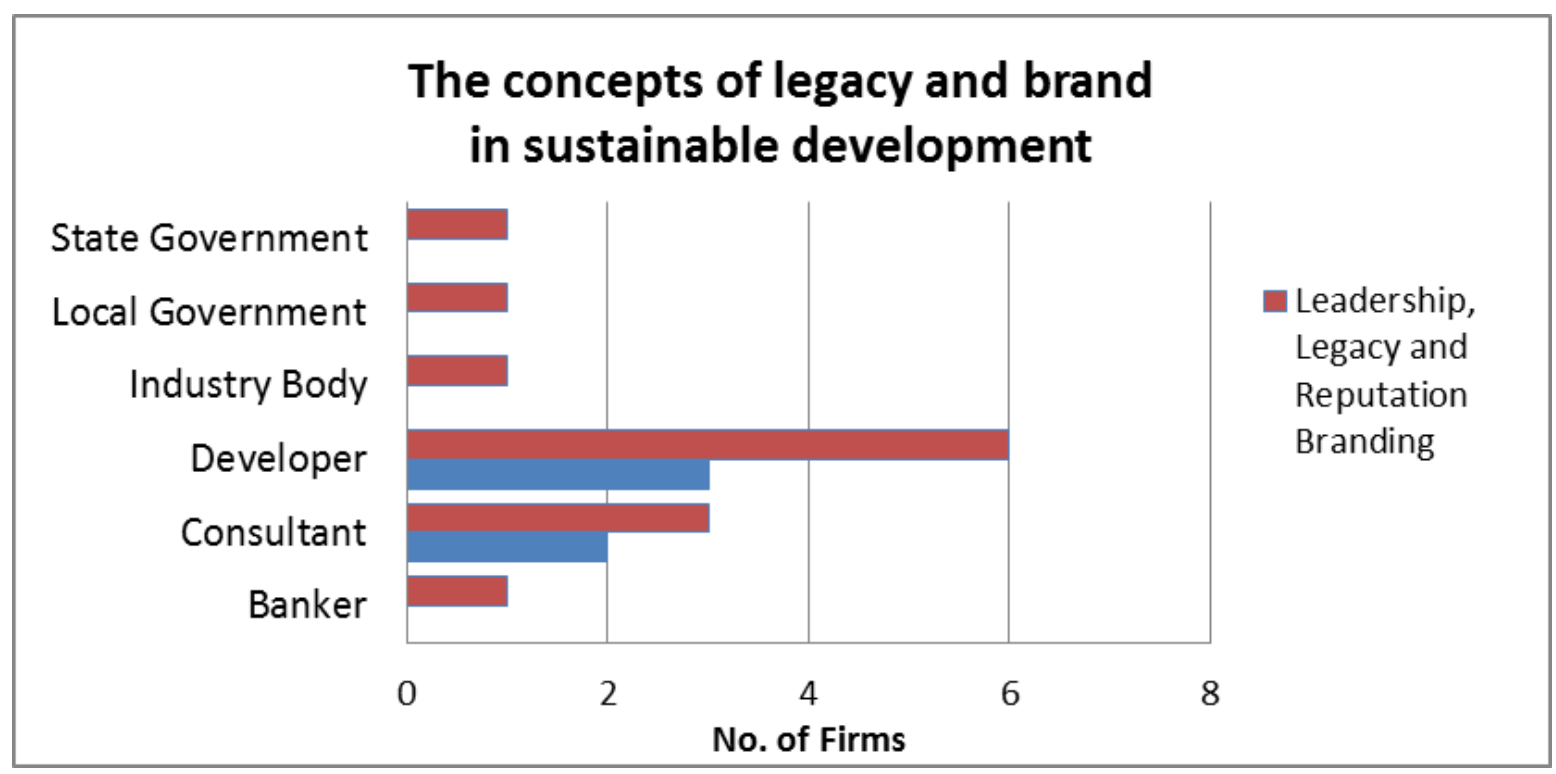

Figure 21 The concepts of leadership, legacy and brand

These concepts were generally related to the scale of the development firm, and the timescale of developments. Legacy was seen as crucial for large, public companies, which were particularly concerned about their corporate reputation and brand. Such companies sought to demonstrate enduring value and quality over time. Some respondents said that large companies were more concerned about the impact of climate change on their brand and their sales, than climate change itself.

"With regard to extreme events, especially floods, the development community is worried about it. <Big developer> was quite proactive with $<$ development on the river $>$, but am not sure if this is really acknowledging the issue or just that they are more concerned about their reputation and brand" (LG1, Local Government).

Larger companies were also viewed as leaders, with a better ability to manage risk and to deliver innovative products. They were seen as very concerned about their reputation, and would not promise products or features that they could not deliver. Many larger companies also had environmental charters, which required them to display leadership, innovation, and deliver against environmental benchmarks.

Nonetheless, in a booming market, many developers, even smaller firms, often innovated, to differentiate themselves from the competition. However, even in a recession, visionary firms still existed; and these sought to produce high quality products. Such firms were generally driven by a "champion" with decision-making power, and often were early innovators, followed in time by the rest of the industry. For example, if a developer produced a particularly innovative product, they entered competitions (i.e. run by the UDIA). If they happened to win an award, other developers viewed the product, and even used aspects of the design in their own developments. 
"He has done a good job down there <an ecologically sustainable development> but no one ever tells you the story about the massive fights that he had with the government to agree to his ideas. So leaders have to have very strong personalities and a real belief in what they are doing to fight the bureaucracy and have the resources to do it" (CS1, Consultant).

Related to brand and legacy, was a general desire by developers to be seen positively as professionals. Developers thought that they were perceived negatively by the general community, as "cowboys" or "the white shoe brigade". All developers interviewed stressed that they were professional; they used professional consultants, and tried to be innovative and bring out good products. They also felt that poor construction standards or other damage were detrimental to the environment and to the development industry. As one developer emphasised, "developers are normal people with families and kids, and we all want to reduce our footprint" (SD1, Small Developer).

Respondents also stressed that they took a significant risk bringing things to market; there was a long gestation period between purchasing a development site, and making a profit (if any) from sales of development products. Moreover, during the entire time, their money and even their own homes, were at stake. This desire by developers to be viewed as serious professionals could be a major driver for adaptive capacity.

\subsubsection{Development Characteristics}

\section{Access to and Availability of Technology and Innovation}

Developers were generally viewed as practical people, and thus wanted practical, implementable solutions rather than theoretical and untested ideals. This finding was not unexpected, given that many respondents had engineering or construction backgrounds. They also felt that any adaptive measures had to be flexible.

It was widely agreed that if property developers were taking on the climate change challenge, they were "badging" it as ESD.

"I suppose that it previously have been environmentally sustainable development. I have never had a conversation with developers about climate change per se. It is more about their response to the challenges of what the market is looking for in respect of climate change, so in my experience it has always even attached to the ESD principles"

(CS1, Consultant).

Some participants did not specifically mention climate change (particularly in the context of sea level rise) but they did couch the response to the risk in terms of ESD and energy efficiency. Further, some business communities were viewed as "highly engaged" in climate change response through ESD principles. Moreover, ESD features were commonly included in commercial and retail buildings, as well as many residential buildings:

"The project has to get a minimum score in the scorecard so it is up to the project to select what sort of initiatives in achieving the minimum sustainability requirements" (LD1, Large Developer).

Often, the underlying rationale for implementing ESD features in a development was not for overt environmental reasons, but to improve convenience and comfort of the buyers. Promoting developments as sustainable was used primarily as a marketing tool to get more sales, rather than from any actual concern about the environment. However, even if developers were personally concerned about ESD, the consumers were not requesting it and nor were they prepared to pay extra for it. 
However, if ESD features are well designed, these can be affordable. Thus, if sustainable developments are branded as affordable, these might be viewed by the market as desirable. Further, if certain sectors of the market (i.e. the retirement sector) demand features such as self-sufficiency for energy, then developers were likely to respond to that market driver; and this may filter to the rest of the residential market.

"People don't really care so much for the environment. Especially with the carbon tax on the nose as well. Why pay extra for this if you are going to get the same sort of house next door in the same community for 20 grand less, is it worth it, not too sure" (MD3, Medium Developer.

\section{Change Use and/or Location of Development}

The ability to change the use and/or location of development combines three of Burton's (1996) adaptive measures; Bear the Loss, Change Use and Change Location. These might involve converting vulnerable areas from development to greenspace, transferable development rights, choosing alternate sites on which to develop, or even planned retreat.

Very few participants mentioned any of these options, except for planned retreat, which was viewed extremely negatively.

"Council at one stage was actively seeking to prevent those people seeking to protect their houses. Thankfully that was overturned in court, but to me that was just an unacceptable condition that people clearly have a right to defend their houses" (CS8, Consultant).

Most felt that if governments tried to force people to move to less risky areas, they would resist, partly because "Australians have a love affair with the coast" (AR1, Architect). In addition, such policies needed to give people options; to what alternative (and equally desirable) areas could they move? Even the sustainability consultants felt that planned retreat was not a viable policy.

However, some respondents did change the locations of their future projects, to LGAs that were seen as more proactive and accepting of development. If possible, developers also chose sites that had fewer risks, and were more profitable to develop. A small developer said it was unlikely they would develop on flood-prone land because all the locals would know about it, and it would cost too much to fill.

Further, subsequent to the Brisbane floods of 2010/2011, some commercial or mixed developers were moving essential infrastructure from basements to higher levels. They were also using the lower floors for retail and offices and higher floors for residences.

\section{Time \& Scale of Development}

A major finding of the research was that the temporal and spatial scale of a given development was integral to whether climate change was considered a risk (particularly direct, physical risk) (Figure 24). For example, "it is all about time. Do you get in and get out, or are you there for the long term?" (SD2, Small Developer) or "timing is integral to risk perception" (SC1, Sustainability Consultant). 


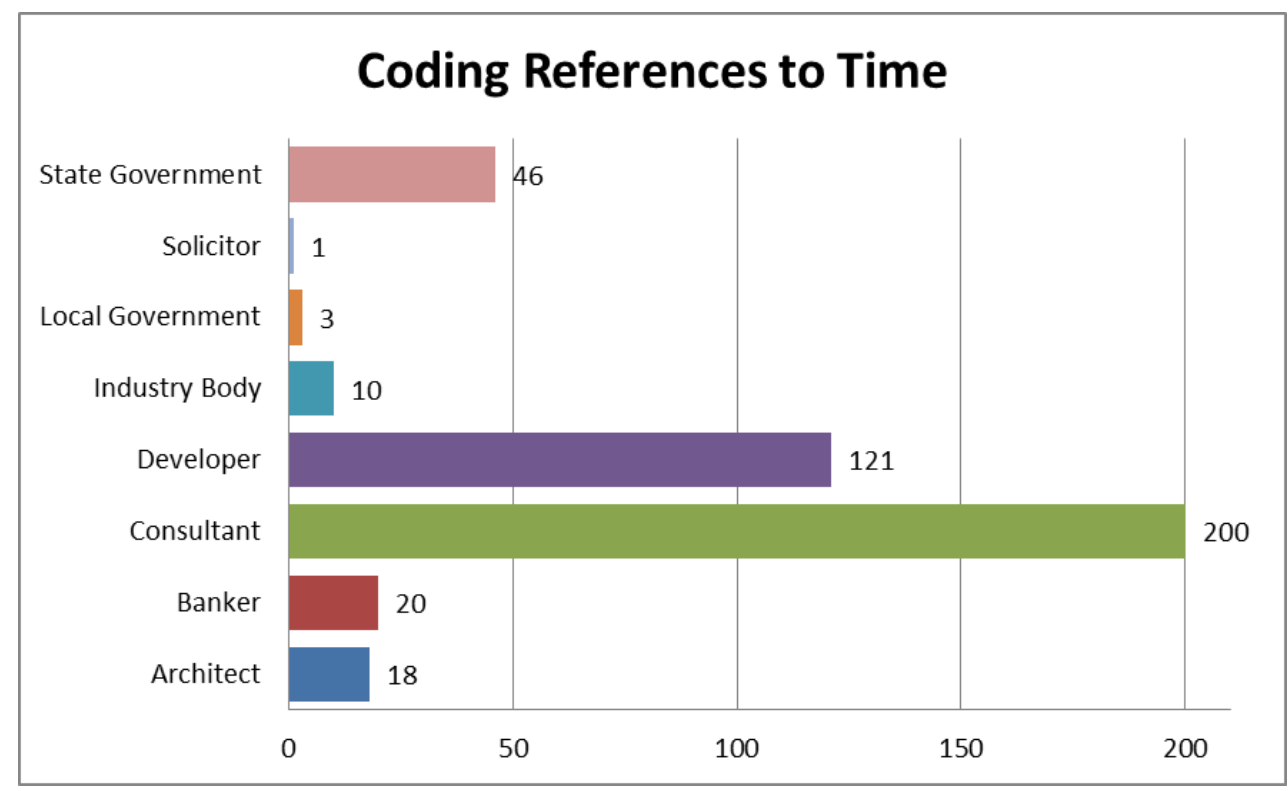

Figure 22 Coding References to Time

Many developers were only concerned with getting in and out as quickly as possible, and paying off bank loans. As the timeframes of their developments were so short, many smaller developers were not concerned about climate change. Moreover, this was exacerbated by the funders; bankers stated that the maximum period for which they would lend money for property development was two years.

The majority of participants considered that the time frames in which they worked were too short to worry about climate change, which they saw as happening far in the future. In the words of one developer, "That adaptation thing is not really at the forefront of developer's minds. Especially with the current impacts of the GFC and that, we have much more important things, in the short term, to worry about things that may happen in 90 years' time, or over a period of time" (MD3, Medium Developer).

On the other hand, institutional investors, such as superannuation funds, took a longer term view, and factored climate change into their projects. However, these mostly funded large scale, commercial projects. Indeed, climate change was seen as a potential risk to bigger developers with lengthy and large projects. Further, bigger developers were seen as wanting to leave a legacy, as their brand was associated with their developments in the long term.

"Some who have projects with a really long time frame, 20 to 25 years, under which they will be developed, have it within their thinking at some stage, in their planning at some stage knowing that they will most probably need to respond in different ways over a number of strategies. The smaller six pack developers mums and dads who want to develop and get out are driven largely by what markets will do and the codes and energy efficiency" (CS5, Consultant).

This was the major difference between residential and commercial developers. Residential developers sought to finish and sell all development projects as quickly as possible, whereas commercial developers built projects to hold and rent to tenants. Of note, many developers built both residential and commercial buildings; and they used the income from commercial buildings to help fund the riskier residential developments (or pay holding costs, such as land taxes and rates) before and during development. 
As commercial developers generally did not generally sell their buildings, they wished to reduce their long term maintenance costs, so ensured that the buildings had energy efficient fixtures and fittings. Further, more sustainable commercial buildings (i.e. high Green Star or NABERS ratings) attracted more desirable tenants, such as government agencies, or large corporations.

However, all developers felt that the time taken by governments to approve developments was excessively protracted. Some wished to undertake short term developments, and the approvals process had resulted in lengthy delays, of a decade or more. This was considered a significant risk, as land had been bought for a good price, with the expectations of making a profit when selling development projects. However, most agreed that, "time is money, and the longer it takes, the more it will cost" (SC1, Sustainability Consultant) Therefore, the cost of holding land for long periods was extremely onerous, due to land taxes, rates, interest payments, etc., and the eventual profit, if any, could be significantly less than initial projections.

\section{Type of development}

The type of development product also had an impact on willingness to implement adaptive measures, and thus adaptive capacity. Type of development is, of course, interrelated with the spatial and temporal scale of development (discussed above) and bank's lending criteria. Large master planned developments, or CBD high-rises take much longer to develop and build, than do a small block of townhouses, or a small subdivision.

The major dichotomy apparent in the research was between commercial (and retail) development and residential development. Developers of commercial buildings were actively implanting adaptive measures, such as passive ventilation, solar panels, recirculated water, water sensitive urban design, and white painted roofs. They were also "competing" to achieve higher Green Star and NABERS sustainability ratings.

There were a number of reasons for this; commercial developers were responding to demands from quality tenants; they were granted government subsidies for doing so; and they tended not to sell their buildings, but keep them and rent them out. Because they did not sell their buildings, they also wished to reduce operations and maintenance costs.

However, with the exception of very large developers, most residential developers were not actively marketing their products as sustainable. This was due to an almost total lack of market demand; desire to sell the development products as quickly as possible; and no incentives from government. Further, some developers reported that the inflexible planning approvals process of many LGAs and the State Government actually prevented them from trying innovative developments.

\subsubsection{Economic and Market Characteristics}

\section{Economic Resources and Financing}

The financial capacity of a development firm can be a driver or barrier to adaptive capacity. A large firm may easily afford to incorporate climate adaptations into developments, whereas the lack of economic resources is likely a barrier for a smaller developer. This study concurred, finding that economic and market conditions were some of the most important drivers and barriers of adaptive capacity.

For example, economic capacity has a major impact on a developer's ability to borrow money, particularly from traditional lenders, such as banks. Banks were extremely risk averse, and were wary about lending money to developers for innovative or new designs, even if sustainable. Therefore, they most often lent money to developers who had previously built and sold similar projects to the one for which they required funding. 
For this reason, some developers purportedly reproduced variations of the same product until the market for it changed.

However, if the development company had sufficient capital reserves, and could demonstrate a high degree of pre-sales, the bank would be more likely to lend them money (assuming any other lending risks had been dealt with).

"We also ask the developer if they have some financial capacity to fall back, can they cover that risk. Because if someone has every last dollar in there, and they rely on this project, albeit the project might be really profitable, if it rolls through storm season for example...I always put a month's contingency" (FI2, Financier).

The financial capacity of developers also determined the sites targeted for development, and the type of development. For example, smaller developers could not afford to practise land banking (retain land for lengthy periods, to develop in future) because of land taxes, rates and other charges. Therefore, they were more likely to be opportunistic when purchasing development sites.

This could influence adaptive capacity because the larger developers generally owned most of the prime sites; and smaller firms could be forced into purchasing lower quality sites, with more climate risk, such as on floodplains. Further, competition for sites between smaller developers might cause a firm to act too hastily, and purchase a site without adequate due diligence. Finally, the high cost of desirable land could force smaller developers further from the city, exacerbating urban sprawl.

Another potential motivation for adaptation to climate change was for bankers and insurers to set market signals, or offer incentives for adaptive developments. At present, these were not doing so, except for insurers in North Queensland, and this may potentially be maladaptation, as some are reportedly no longer insuring their property because of high premiums. Shareholders of large companies could also pressurise developers to implement adaptive measures.

\section{Insurance Industry}

The insurance industry can also be a major driver (or barrier) to climate change adaptation (as discussed in Chapter 3, Climate Change and the Private Urban Development industry). Extreme weather events can have very high costs, in money, property damage and lives. Since 1980 in North America alone, weather catastrophes have incurred a total cost of US $\$ 1,06$ billion, with US $\$ 510$ billion in insurance losses, and 30,000 deaths (Munich RE, 2012). In Australia, the cost to the insurance industry of the 2010/2011 floods in Queensland and Victoria, and Cyclone Yasi, was A\$6.6 billion, with an insurance cost of $\mathrm{A} \$ 4.26$ billion (Insurance Council of Australia (ICA), 2011).

In response to these events, the insurance sector response to risk management has shifted from 'reactive risk' management to 'rational action' (Munich Re 2010). For example, insurers in areas such as North Queensland have already increased premiums, in some cases, such as for strata titled units, by greater than $300 \%$ (IAA 2011, p.2).

These price rises have not yet filtered down to SEQ, but in future, severe weather events are likely to pressurise insurers to follow suit. For example, the ABCB has recommended moving the $\mathrm{C} 1$ cyclone limit from Bundaberg to Caboolture, which would incorporate the Sunshine Coast (population 340,000) and potentially lead to insurance premium increases and increased building costs to comply with the Category 4 cyclone ratings (Australian Building Codes Board, 2012).

Therefore, an increased risk of weather disasters from climate change presents significant monetary and reputational risks to the insurance sector as well as some

106 The capacities of private developers in urban climate change adaptation 
opportunities (Mills, 2009). The insurance industry has the potential to be a major driver of adaptation. For example, insurers could link premium rises to the provision of adaptive measures. This could encourage developers to incorporate these into developments, or to retrofit existing developments, particularly commercial developments. Further, in certain areas or market segments, insurers will not even insure properties, as they believe the risks and costs of doing so are excessive (IAA 2011, p.16). This could lead in the market purchasing elsewhere.

"Until there are financial values put on this...so this is the financial value of not acting, and this is the financial value of acting, until that level of detail comes through it is going to be challenging for businesses to be proactive in responding to climate change risks. I think that it would be great to see some research in that field to come through, from recent examples such as the Queensland floods. I think that the insurance industry can also drive it. Business responds very well when they are able to see the dollar value of a risk or an issue rather than a theoretical impact of what may or may not happen" (LD1, Large Developer).

However, insurance industry practices can also result in maladaptation. For example, extremely high prices may lead to none, or underinsuring of vulnerable properties. These could also negatively impact the development industry; for example, buyers may be put off purchasing strata titled properties due to high insurance adding to body corporate costs. This may be argued as discouraging development in vulnerable areas, but in smaller, regional areas, could have a strongly negative impact on the property industry, and on a region's economy. Arguably, this has already occurred in some areas such as Airlie Beach and Magnetic Island (off Townsville) (Studdert, 2012).

This study found a degree of complacency from most respondents, particularly developers. The general consensus was if construction or infrastructure were impacted by severe weather events, it was not an issue, as the risk was transferable to the contractors or the developer's insurance.

"Extreme weather events are natural, but we are not concerned because they are covered by insurance. It is a corporate and bank requirement to have insurance" (MD3, Medium Developer).

However, the ultimate insurance risk was borne by the lenders, as a mandatory condition of loans is developer insurance. However, a Sustainability Consultant said that despite this, few lenders actually check if the developer has sufficient insurance. Insufficient insurance cover could lead to serious litigation issues if bankers don't check on developer's policies, and a major climate event occurs.

Some felt that insurance would be an issue in the future, but it was not driving anything at present. They also felt that if different sites had different insurance criteria, or some sites became uninsurable, that would have a major impact on the industry. One large developer said, as far as he knew, no developers had storm surge insurance, and very few insurers offered it.

Maladaptation may also occur when governments, insurers and industry regulators send the "wrong" message to consumers and developers about climate change (Crichton, 2003). When government intervenes to ensure that people are covered by flood insurance no matter where they are located, the result is that more people are going to move to that place and consequently the number of those exposed to floods in the future increases (Crichton, D; 2003).

\section{Market conditions}

Markets and marketing are critical and central to development, as there is no point in developing products that do not sell. Therefore, market conditions are arguably, the key 
factor that influences development, and how developers adapt (or not) to climate change. Developers are likely to respond to market signals and to consumer preferences. Market forces, such as property booms, can be a major driver of development; and recessions or localised economic issues, can be a major limiter of development. In addition, property booms can drive innovation, with the inclusion of sustainability features, used as a point of difference for competing developers. On the other hand, recessions, such as the GFC, can result in developers cutting sustainability features to save costs.

In this study, the willingness to adapt was largely dependent on the cost-benefit of the adaptation-any adaptive measure had to have positive cost benefits and returns on investment for developers. For example, if the market did not see a tangible value in an adaptive measure-i.e. a house built to higher cyclone rating than required by lawthen they were not prepared to pay for it, and the developer would lose money. On the other hand, if they could see a tangible benefit-i.e. a water tank allowing them to maintain a green lawn-then they were prepared to pay for it. Developers also said that customers found it difficult to comprehend the long term benefit of certain adaptive measures, as they did not intend to remain in the property for longer than $5-7$ years.

It was thought that unless a financial value or other incentives were put on climate change adaptation, nothing would happen (or would happen very slowly). Business usually responded well when given the dollar value of a risk or an issue, rather than theoretical or intangible threats. Only if developers could successfully brand and sell climate resilience as something for which there was willingness to pay, could the market drive change in the industry. However, "people don't care about the environment if they have to pay extra for it" (MD3, Medium Developer).

Further, developers did not like to spend extra money on adaptations, and tended to choose the easiest and cheapest possible under law. When purchasing sites for development, and if two sites were the same price, they usually chose the less risky site, unless the riskier site had a better chance of return. Further, developers did not consider it cost effective to build to a higher standard (i.e. higher cyclone standards on the Gold Coast), when they felt that the chances of this happening were minimal. However, cyclones and severe tropical lows regularly impact SEQ, and if a Category 4 cyclone crossed the coast, there would be massive property damage, and a huge impact to the Queensland and Australian economy.

In general, it was thought that eventual changes in market demand (as have been seen in commercial development) would force developers to change, but this would take too long. Therefore, it was essential that the power of regulation was used to promote adaptation.

\subsubsection{Regulation, Legal and Governance Characteristics Developer Standards}

As discussed in Chapter 5, many property developers, and allied organisations have developed various voluntary industry-specific codes, standards and measures to address the sustainability of property development. These schemes include reporting tools such as Corporate Social Responsibility (CSR); the Green Star and the National Australian Built Environment Rating System (NABERS) rating systems; and the EnviroDevelopment certification scheme. For example, industry certification tools, such as EnviroDevelopment, are encouraging developers to take a sustainable approach to property development by incorporating a range of sustainable practices with respect to waste, energy and materials. Although EnviroDevelopment does not brand itself as a tool to mitigate climate change, many of its proposed practices carry ancillary benefits to climate change mitigation and thus need to be recognised in the context of climate change mitigation. 
This research found that nearly half (48\%) of survey respondents used the Green Star system, and to a lesser extent, NABERS and EnviroDevelopment. These schemes were commonly used by many interviewees and focus group participants. All participants viewed them positively.

"It would be useful to take that general market perception and how to translate into real benefits and the Green Star - because you can say there is a real benefit here, we are delivering extra environmental goodies, and there is an independence to that and people are convinced that it is real" (MD2, Medium Developer).

However, most schemes are used in commercial development, and only to a lesser extent in residential development. A strong commercial demand existed for Green Star and NABERS ratings, driven by tenant demand, lower operating and maintenance costs, competition between developers for ratings, and government subsidies. Developers said these ratings helped attract quality tenants, who demanded them.

Industry self-regulation thus appears to be a potentially valuable driver for sustainability and increased adaptation to climate change. Of note, little demand for "green" buildings was reported as coming from the residential sector. Therefore, only the largest developers introduced substantial sustainability initiatives into residential developments. Some demand for sustainability initiatives was noted from the retirement sector, based on a desire to save money. If this could translate into the wider residential sector, then the existing industry support for such tools, could be a valuable adaptive measure.

\section{Legal and Litigation}

Like insurance, litigation has the potential to be a driver or barrier, to climate change adaptation. For example, developers may be liable for legal consequences, if they develop in areas vulnerable to climate change (UDIA (NSW), 2008). Some legal disputes have already occurred between developers and LGAs in coastal areas deemed at high risk for inundation from sea level rise (Briggs et al., 2010; Fitzgibbon, 2010). To date, these lawsuits have resulted in maladaptation rather than adaptation, as the residents of areas, such as Belongil Beach in NSW, prevented the LGA from enforcing a planned retreat policy (Perinotto, 2012).

In this study, respondents were largely unconcerned about the possibility of litigation. They felt that if they adhered to the BCA and LGA standards and planning regulations, they would not be held liable for legal action. A consultant said such companies were protected by law; and as they always worked in accordance with the law, and standards, they were protected against litigation. It was also stressed the engineers are very tightly controlled by law and professional standards, and as a group tended to be extremely cautious.

A sustainability consultant said that property developers largely did not recognise the potential litigation risks that could result from climate change. However, in Queensland, there were no legal precedents for litigation based on negligence of climate change risk. This was emerging, but was as yet, untried. The consultant also felt that the nexus of climate change and property development wold be determined in court, and would come down to who had the best lawyers (i.e. firms with the most money) or those who could successfully lobby government to change the law.

This is not to say that developers and consultants were not sued; this happens frequently. Litigation between developers, consultants, local and state government and proponents is frequently argued in the Planning and Environment Court. Such cases are not overtly about climate change, but if a site was flood prone, and not specified as such, a developer could sue the local government for loss of value. 
Where cases have been argued in court (for example, Belongil Beach in NSW), the residents of properties at risk (in this case, an upper income area that the Byron Shire Council wished to resume under a planned retreat scenario), sued the LGA. At present, the legal environment favours private property rights; and if property owners thought governments were inadequately defending their properties, they often took legal action.

\section{Regulation}

The regulatory regime can also be a major driver or barrier to adaptive capacity. Regulation aims to manage development in a number of ways including: prevention and removal of development; regulation of some market aspects; and engineering and building codes. For example, development may not be permitted in certain areas, and according to specified rules; such as below $5 \mathrm{~m}$ AHD on a frequently inundated floodplain. Regulatory policies require a developer to adhere to certain conditions, such as density and site coverage; and to implement environmental management plans. Compulsory regulations are often backed by punitive measures, such as penalties.

Regulatory drivers, such as changes to coastal planning policy, are not voluntary, and are often developed by the public sector, with little private sector input (Taylor et al. 2012). This can lead to industry dissatisfaction, and may result in companies suffering financially, choosing not to develop, or even resorting to illegal practices to circumvent the policy, particularly if decision makers are sceptical about climate change.

This study found that respondents held markedly different views on regulation: some thought that regulation would drive adaptation, whereas others thought the market would drive adaptation. The latter assumed that private companies, such as bankers, insurers and developers, would be the main drivers of change, then the government would follow suit. To some extent, this is supported by the findings; most evidence of adaptive measures (i.e. insistence on Green Star rated commercial buildings) was driven by the private sector, and not by the regulators.

Regulatory issues were also seen as barriers to adaptation; some felt that the degree of regulation was inversely proportional to the degree of innovation. One significant barrier was the time taken to approve development. Respondents felt that if regulators took twice the time to approve an innovative development, then they were not prepared to take that risk again. If their development took twice as long to build as the developer's competitors, this was seen as a major disincentive.

The lack of information from government was also seen as a barrier. Some were sceptical about government risk modelling, emphasising that much government data are regional assumptions, and not locally specific. As an example, a consultant had analysed the use of shopping centres as designated refuges during climate events, and had found that the owners were generally unaware of building's status as a designated refuge. Moreover, the construction and facilities were largely inadequate to protect a large number of people during a major event.

"You are very heavily reliant on what you are told about what the parameters are; the risk of flooding or your risks of that particular location. You are relying on the planning agencies to have the right knowledge, and they will give you the right information...because the knowledge is not in the hands of the developer. They don't generally have the scientists employed. They are not looking in 30 year's out, they are looking in the planning framework, which says you can build within these guidelines. And these guidelines are not very clear" (LD2, Large Developer).

Other respondents thought that regulation and not the market, would drive adaptation; and that developers would not do anything more than the minimum required by the regulations and BCA unless there were a market advantage in doing so. Moreover, if

110 The capacities of private developers in urban climate change adaptation 
they felt a regulation significantly disadvantaged them, some large developers and the PCA reportedly lobbied the government to change the law. Despite this, some of these respondents felt that adaptation to climate change would eventually be mandatory, and eventually all developers would have no choice but to conform.

An innovative driver suggested by a consultant, was to put flood hazard zones on rates bills or property titles. This might dissuade people from buying in certain areas (either from perceived threats to property, litigation, insurability, or capital growth).

\subsubsection{Putting it all together: Systems}

As indicted in the previous sections, a number of potential indicators of adaptive capacity exist. To a greater or lesser extent, all these indicators are intercorrelated:

"The property development is one cog in a large world. They are so connected to everything else. They are connected to government regulations to local government, they are connected to supply chain and to lending and insurance risks and I think that we need to have more of a systems' way of exploring adaptive capacity" (SC2, Sustainability Consultant).

Further exacerbating the complexity of the environment in which developers operate are the actions of local LGAs. For example, one developer spoke of purchasing a development site with no historical record of flooding. However, the LGA then changed the management of the local dam, with the subsequent result that it partially flooded the site, thus reducing its value; thus it is also important to consider, "how local authorities manage urban systems (such as dams and waterways) in the context of climate change" (MD1, Medium Developer).

Ignoring this complexity will likely result in maladaptation, rather than improving adaptive capacity. Thus it is likely that the adaptive capacity of the property development sector will be a complex, multi-dimensional, interrelated system, rather than a collection of indicators.

Therefore, Figure 25 (below) has been adapted from Table 8 (General Measures of Adaptive Capacity). The measures of adaptive capacity have been differentiated into Participants, Internal Characteristics, Development Characteristics, Market Characteristics, and Regulatory Characteristics.

Below, two hypothetical case studies are given to illustrate how a developer could respond to a direct climate threat (a cyclone) or an indirect climate threat (a new policy aimed to adapting to sea level rise). For each threat, the characteristics of a hypothetical developer with strong adaptive capacity, and a hypothetical developer with weak adaptive capacity, are described. Of note, these are fictional examples, used for illustrative purposes, and they do not refer to any developer, known or interviewed, in SEQ or elsewhere. 


\section{Physical Threat - A Category 3 Cyclone impacting SEQ}

After weeks of heavy rain, a tropical low forms to the north-east of Fraser Island, and begins to track in a south-westerly direction. As it moves closer to the coast, it intensifies to a Category 3 storm, and crosses the coastline in the vicinity of Southport. The cyclone coincides with high tide, and a major storm surge is experienced in lowlying areas of the Gold Coast, Brisbane and Sunshine Coast. Significant property and infrastructure damage occurs due to the high winds and storm surge.

ABC Firm is a large developer, with a diverse property portfolio, including a new, mixed use (retail and residential) high-rise building next to the ocean on Main Beach Parade. The storm surge and Nerang River flooding isolates the area, preventing access. No wind damage occurs, save for some broken windows on the lower level due to flying rocks from nearby landscaping. The lower levels of the building, including the car park, are flooded. Further north, XYZ Firm is a small developer, with a new townhouse complex near Hope Island. Likewise, the complex is cut off by flooding from the Coomera River. Although it is less impacted by storm surge, significant wind damage occurs, from roof losses, and water entry into inadequately sealed windows and doors.

As part of their development, ABC Firm have prepared for events such as the cyclone, and no essential infrastructure (such as air-conditioning or lift mechanisms) are located in the basement. They also have an emergency preparedness plan, with an early warning system so residents can evacuate the area prior to the cyclone. Because they have also introduced sustainability measures and higher rated construction, the building suffers very little damage, for which the company is already insured.

However, XYZ Firm acquired their development site in a flood-prone area, because they did not have the financial capacity to compete with larger developers. The development was running behind schedule, and the banks were insisting on early repayment of loans. The developers told the contractors to cut corners to speed up the process. The development suffered major structural damage from flooding and wind, mainly due to cheaper materials used and poorer construction quality. The firm could not afford full insurance, so their policy did not cover most of the damage. In the aftermath, the firm entered into liquidation, with the townhouses left incomplete.

In this case, the larger developer (Firm Size) had a greater capacity to cope with an unexpected climate event such as a cyclone. They had greater economic resources, and are less reliant on finance, so were more resilient, and can recover faster (Economic and Market). Further, ABC Firm's high-rise was a very large development, which took substantial time to plan, build and sell. ABC Firm was well aware of the potential risk from storm surge (Internal) and incorporated adaptive measures, such as flood retention barriers and relocatable infrastructure into the building (Development). The firm could afford to do so, because of its large and varied property portfolio (Economic and Market).

$A B C$ Firm were also very concerned about leaving an ongoing legacy, and any impacts on their brand and thus sales (Internal). Therefore, they hired specialist consultants to advise on their statutory and regulatory requirements. They also subscribed to Green Star and NABERS ratings for their development (Regulatory).

On the other hand, XYZ Firm had poorer adaptive capacity. They had fewer resources, and were almost completely reliant on the bank to finance their development (Economic and Market). Thus, they could not afford expensive technological measures or to build to a higher standard than the BCA and LGA regulations; thus their development was significantly damaged (Development). Moreover, they only incorporated the very minimum required by law, and because of financial pressure, the subcontractors cut a few corners (Development; Regulation). 


\section{Indirect Threat - Onerous Regulatory Change}

After a series of severe weather events, a new State Government is elected with a climate change mandate. They rapidly introduce a new policy which requires Local Governments to designate areas for planned retreat. Instead of engineering structures to counter coastal erosion, planned retreat allows for the relocation or removal of development with the landward movement of erosion. Many areas already subject to coastal erosion are listed; mass outcry is heard from the development industry.

PQR Firm is a small boutique property developer, specialising in high end apartments. The owner of PQR Firm had previously worked for larger developers; and also has a particular interest in the risks of climate change. She has entered and won a number of UDIA competitions, and all her properties are EnviroDevelopment certified. She cannot afford to hire specialist consultants, so conducted her own research into coastal hazards. Because she is highly networked, through the UDIA and other organisations, she already knew about the new policy, and even commented on preliminary drafts.

HIJ Firm is a medium-sized property developer, which has been in business for many years. They employ their own engineers and building contractors, and also subcontract work to consultants, typically planning consultants from established firms. They are currently redeveloping an old marina, with apartments, retail and tourist operations. HIJ has low staff turnover, and their management team have been in position for a long time. They are a traditional company, and their philosophy is "tried and true".

The owner of PQR Firm has conducted research into similar policies, and hires a specialist architectural firm to incorporate adaptation and sustainability measures into the development. The residential building is situated at the landward side of the lot, further than $20 \mathrm{~m}$ from the coast. The beachside part of the lot is landscaped with dense native bush, and incorporates natural drainage lines. The swimming pool is also designed to be flooded, and easily drained. As the development is so well designed, it has a high proportion of pre-sales, so she easily obtained funding from a major bank.

On the other hand, HIJ Firm is more restricted; the site is already developed, with existing infrastructure. They will not locate the buildings at the rear of the site, as they feel the market will pay a premium to be "on the water". They want to attract the yachting fraternity, and want the hotel to be next to the marina. The new policy will significantly reduce their property values, and potential sales. In response, they employ specialist lobbyists to persuade the government to water down, or overturn the policy. If this is impossible, they want a moratorium on existing development, and the policy only to be implemented in 2020 (when a new government might be more cooperative).

In this example, PQR Firm has greater adaptive capacity because the owner has greater awareness, and access to information (Internal). The development incorporates the latest technology, and is flexible, with the ability to change use (Development). The owner is aware of regulation changes, and follows industry standards and certification (Regulatory). Specialist Consultants create an innovative design, thus there is good market demand, so financiers loan the money, and the insurers charge reduced premiums because of the adaptive measures (Economic and Market).

In contrast, HIJ Firm is a lesser degree of adaptive capacity. They are sceptical about climate change, and do not keep up to date with research (Internal). They use their own Consultants, who are also sceptical. The marina is similar to other, previously successful, developments, but it is very inflexible, and does not incorporate any innovative technology (Development). They lobby against the new policy, and consider commencing litigation against the government; nor do they subscribe to any voluntary industry standards (Regulatory). Their financiers and insurers impose strict conditions and high premiums after the new policy is announced; and this seriously affects demand for the development products (Economic and Market). 


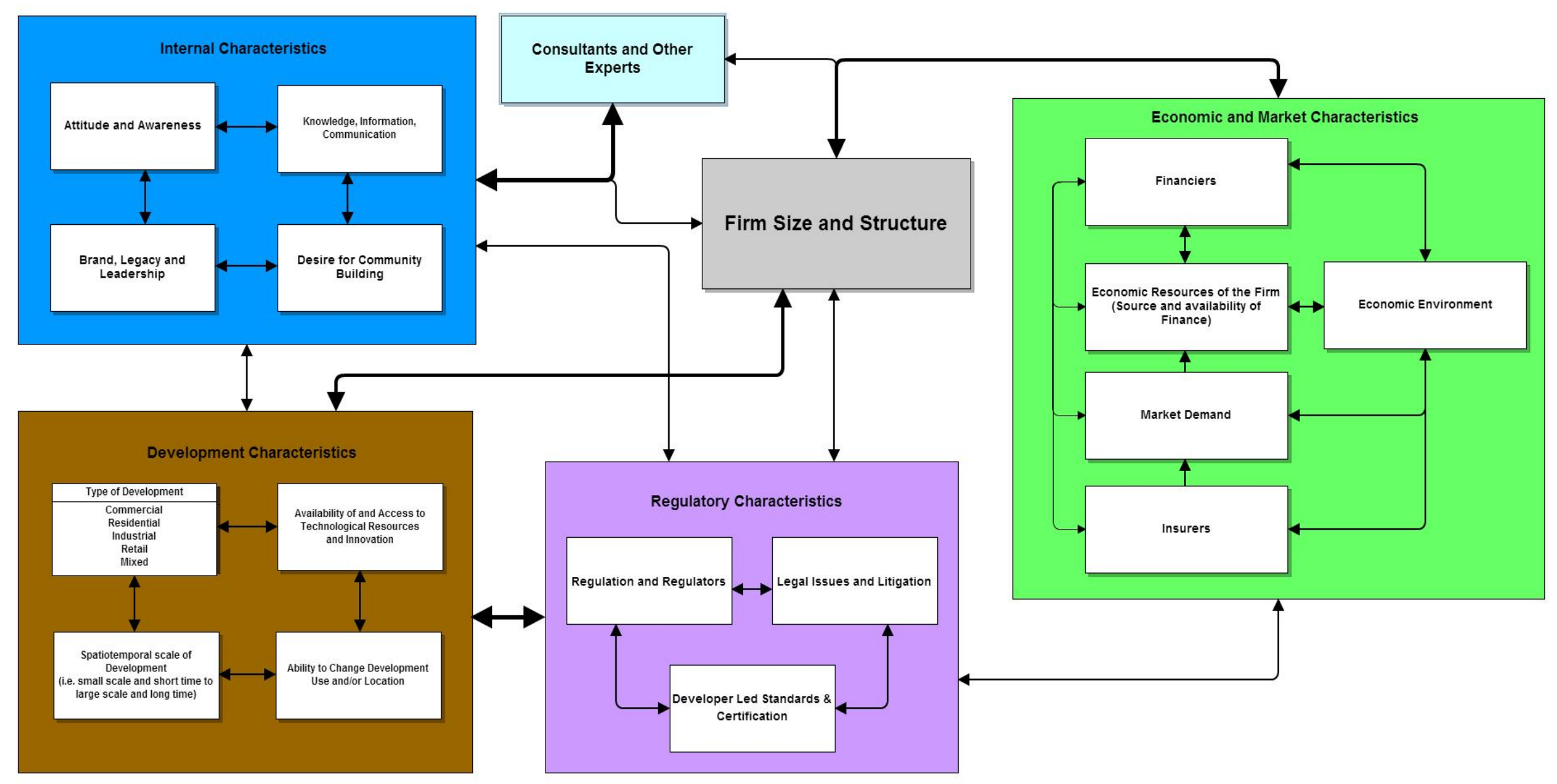

Figure 23 Schematic of Measures of Adaptive Capacity

114 The capacities of private developers in urban climate change adaptation 


\subsection{Conclusions}

Many respondents had already implemented adaptive measures into their developments. These were generally technical or engineering measures, such as raising floor levels and moving critical infrastructure out of basements. Most respondents said they took into account site location, climate resilient infrastructure and long term planning. Interestingly, no large developers surveyed said they intended to incorporate adaptation to future climate change into decision making or current operations. However, this may be due to the fact that large developers already include sustainability measures in their developments.

Adaptive capacity was seen as a flexible, dynamic combination of interrelated determinants, that respond differently, according to a range of places and scenarios (Adger et al., 2007; Smit \& Pilifosova, 2003; Smit \& Wandel, 2006) IPCC,2001). The research found that a range of possible drivers and barriers to the adaptive capacity of the property development sector existed.

These factors could be grouped under five categories: Participant Characteristics: (Developers and Consultants/Experts); Internal Characteristics: (Attitude, Communication, Information and Skills, Community and Society, and Leadership, Legacy and Brand); Development Characteristics: (Access to and Availability of Technology, Ability to Change Use and/or Location, Time and Scale of Development, and Type of Development); Governance and Regulation Characteristics: (Developer led Standards and Certification; Governance and Regulation; and Legal); and Economic and Market Characteristics: (Economic Resources of a Firm, Economic Environment, Financiers, Insurance Industry, and Market Demand).

Of note, these factors are not discrete, but are complex and interconnected. For example, any factor can act as a barrier or a driver, depending on the firm, the current state of the economy, or the regulatory environment.

"Drivers are what the market wants, what the developer thinks the market wants, and what the regulator wants" (SG1, State Government);

"There are probably a few responses and the responses are difficult, they are not easy, because they need to challenge established cultures. That means the established culture of the market, as well as cultures within government and councils" (CS4, Consultant).

The most important drivers of adaptive capacity were economic and market characteristics, participant characteristics and internal structure, and regulatory characteristics. However, these drivers were all interconnected; market demand and economic resources were related to development type and spatiotemporal characteristics, as well as to firm size and structure.

In the post GFC environment, the current market conditions and the availability of finance largely dictated the type of development produced, particularly for smaller firms. For example, if a small development firm was highly leveraged, sometimes with their own home as security for the development, they were less able to risk producing an innovative, different product that may not sell. Further, larger developers with a range of diverse products had more flexibility to introduce adaptive measures, as they were less dependent on bank funding. Such firms were also conscious of their enduring legacy and brand, and did not want to produce anything that would impact on this. They could also afford to employ or contract specialist consultants.

The regulatory environment was just as likely to be a barrier as a driver of adaptive capacity. The major barrier posed by regulation was the time taken to approve innovative developments. The legislative and policy environment was viewed as too complex, inflexible, and unable to deal with issues that fell outside government codes. 
Respondents felt frustrated dealing with a system (i.e. legislation) that did not have the capacity to respond proactively and flexibly to the issues of climate change: "The market and councils limit going ahead with great ideas" (MD3, Medium Developer). They also saw too many overlaps between government departments and responsibilities. The (now superseded) Queensland Coastal Plan was almost universally criticised, as reactive, implemented too hastily, and too complicated.

More onerous regulatory measures, such as prevention or removal of development (i.e. planned retreat) were viewed extremely unfavourably; with many saying that these left LGAs and the State Government liable for litigation. However, many thought that regulation could drive adaptation, particularly in difficult economic times. In good economic times, developers frequently introduced innovative features, as a point of competitive difference, but in poor economic times, they (and the market) were primarily price conscious.

The BCA was the most cited legislation for managing the risks of development. Interestingly, one respondent commented that single climatic events could drive rapid changes in the BCA; i.e. a "tornado" in the southern suburbs of Brisbane (1980), the Brisbane floods (1974 and 2011), and Cyclones Althea (1971), Tracy (1974), and potentially Yasi (2011). Very few respondents mentioned the Carbon Tax, and none felt it had any impact on their business.

The time, scale and type of development were major determinants of adaptive capacity. The larger the development, and the longer the development time frames, the more likely it was that developers were concerned about climate change, and would introduce adaptive measures. The converse was true for smaller developments with shorter time frames. For these, the main goal was to "get in and out as quickly as possible, and pay the bank back" (CS5, Consultant). This was exacerbated by financiers, as banks would not lend money to developers for more than a maximum of two years.

Another potentially significant driver for adaptation was the widespread support by industry for voluntary certification and rating schemes, such as Green Star, NABERS or EnviroDevelopment. Many commercial and retail developers used these schemes in their developments and introduced adaptive measures into buildings. The motivation for this included: reduced operating and maintenance costs (these developers tended not to sell their buildings, but lease them out); attracting quality tenants (i.e.

government agencies); government subsidies and incentives; and competition between developers.

On the other hand, most residential developers were not introducing adaptive measures, other than those required by law, such as rainwater tanks ${ }^{14}$. Some of the larger developers did incorporate sustainability features, but reported virtually no market demand for these. This lack of market demand was seen as "across the board", from detached houses in the suburbs, to high income units on the water. This was thought to result from the GFC and current economic conditions; with the result that purchasers were extremely price conscious. It was also seen as associated with the intangibility of some adaptive measures; purchasers could not see any long term value in these features. In addition, residential developers were not interested in the development after it was sold, and had moved on to the next development.

\footnotetext{
${ }^{14}$ Of note, in December 2012, the Queensland Government flagged that they will remove this requirement in 2013 .
} 
One potential avenue for introducing sustainable or adaptive measures into developments was in the retirement sector. Some mentioned that this market were requesting features such as solar panels and passive cooling; largely driven by the recent increases in energy prices, and the desire to save money. Of note, there was residential demand for energy ratings in areas with more extreme climates than SEQ, such as Canberra.

There were few current drivers for adaptation from the insurance industry, although this sector is very active in climate risk analysis at the corporate level. No respondent in SEQ spoke of insurance premium rises, even if their properties had been flooded in 2010/2011. Nor were any insurance companies requiring adaptive measures. However, this was not the case in North Queensland where, since 2007, premiums on strata titled units had risen by over $300 \%$ because of Cyclone Yasi and storms in Mackay (in 2008). However these rises in policy prices were not linked to any requirement for adaptive measures. Without such, dramatic price rises may promote maladaptation rather than adaptation, as high prices may lead to underinsurance in areas already vulnerable to climate change. ${ }^{15}$

Other practices of the insurance industry could also promote maladaptation. Many were unconcerned about the direct risks of climate change, because they felt that insurance would mitigate any risk; and if a severe weather even occurred, the insurers would cover any damage. Further, one respondent stated that they may not move infrastructure (i.e. air-conditioning units) from basements, because when the next flood came, it would probably be time to replace these; and then the insurers would pay.

Given that these determinants of adaptive capacity are so complex, and interrelated, some respondents felt it would be better to view this from a systems perspective, rather than a "tick box" list. For example, a developer told of a change in dam management that flooded a previously flood-free site. Therefore, no matter what the adaptive capacity of a given property developer, external issues totally beyond their control could have a negative impact. However, adaptability is not a static measure:

"And adaptive capacity changes all the time. You may be resilient one week but nor resilient the next". On a rather sombre note, he concluded, "it <adaptation> probably needs a whole <of> society response. And at the moment I don't think it is a possibility" (SC2, Sustainability Consultant).

\footnotetext{
15 Of note, since this initial drafting of this report, subsequent floods occurred in Queensland in early 2013. Media articles have also reported substantial raises in insurance premiums for certain research. This is a fruitful area for further research.
} 


\section{CONCLUSIONS, RECOMMENDATIONS AND FUTURE RESEARCH DIRECTIONS}

\subsection{Conclusions}

Climate change presents a number of risks to developers because of its complexity and uncertain impact from a range of direct and indirect sources. Most climate change research investigating urban socio-economic and institutional contexts has focused on household and community vulnerability, and on governance and policy. There is however a major policy and knowledge gap in social scientific understanding of private sector developers and financiers' capacity and capability to respond in an effective and timely way to the climate adaptation challenge.

Current and future development decisions and the flows of investment that follow from these decisions will strongly shape future urban capacity to respond and adapt to climate change impacts. Improving the knowledge of the capacity and weaknesses of private urban development and financing institutions in the area of climate change is thus a critical imperative within a wider policy of responsive climate change adaptation. Ignoring the private development sector and its institutional complexities would be to ignore a major potential source of improved adaptive activity.

To this end, this research was undertaken with a variety of South East Queensland (SEQ) property developers and allied professionals, such as consultants and financiers. The key findings of this study were as follows (also summarised in Table 9):

- The development sector in SEQ was highly diverse, as were the types and location of development and sources of funding.

- In general, knowledge of climate change, and awareness of the degree and likelihood of risk, was relatively poor; many associated it only with sea level rise.

- The economic and market environment post GFC had a significant influence on all aspects of development; influencing the provision of adaptive measures (no market demand) and acquisition of finance (conservative lending policies).

- Many did not consider the direct (physical) impact of climate change to be a major threat. However, indirect impacts, such as changed legislation, and more onerous planning requirements, were viewed as more serious threats.

- Most LGAs and some State Government agencies were seen as reactive, inefficient, and hindering innovative development.

- The greater the spatiotemporal scale (size and duration) of a development, and the size of the development firm, the more likely the provision of adaptive or mitigation measures.

- The commercial sector was actively incorporating "green" features into buildings, due to tenant demand, competition for star ratings and government subsidies; but no demand for such features existed in the residential sector.

- Adaptive capacity therefore could be categorised in the following:

○ participants (i.e. developers);

- internal characteristics (i.e. attitude and brand);

- development characteristics (i.e. spatiotemporal scale);

- governance and regulation (i.e. legislation); and

- economic and market characteristics (i.e. economic resources and financiers).

118 The capacities of private developers in urban climate change adaptation 
The SEQ development sector was highly heterogeneous, with a wide diversity of firms and development products. Nearly all developers used consultants (especially planning consultants to prepare development applications). Consultants undertook a range of roles, from specialists to development coordinators; and were regarded as an important source of expertise, to provide extra "checks and balances" to further reduce risk.

In general, the SEQ development industry did not consider that climate change posed a serious risk to them. Most felt that their exposure to risk could be managed by adhering to government regulations and the BCA, and by using insurance. Larger developers and sustainability consultants generally viewed climate change as posing a greater threat to their development operations than did small developers and planning consultants. This is likely to represent the spatial scale of developers and developments; the scale and duration of a development project was directly related to the size of a firm, and the degree to which a developer considered climate change a risk. However, most participants sought to complete and sell development products in as short a time as possible. This haste was exacerbated by financiers, restricting loan terms to a maximum of two years. Thus, the time and scale of development were major determinants of adaptive capacity, in the way that developers perceived climate risks.

From approximately 2000 to 2007 , property development was a relatively low risk activity, due to a booming property market and good capital growth, which resulted in sustained profits. Finance was extremely easy to obtain, and many inexperienced players had entered the property development industry. However, the GFC had a major impact on the industry, with many firms (and financiers) exiting the industry. As a result, financiers are now extremely risk averse, and unwilling to loan money on new or innovative products. Financiers have also significantly tightened lending conditions, requiring higher pre-sales (>75\%), a lower LVR, higher proportion of equity, and increased due diligence studies. Therefore, although banks still provide the bulk of finance to developers, some now obtain finance from high net worth individuals, other business activities and even international investors.

Nonetheless, lending institutions could potentially be a driver for adaptation, by refusing loans for high risk properties. However, financiers did not consider climate change as a risk, particularly given the short period over which they financed development. Bankers also assumed that climate change risk would be dealt with by consultants and regulators in the development approval stage. Further, nearly all respondents (including financiers) considered that any climate change risk to the site, infrastructure or buildings, would be covered by the developer and the builder's insurance policies.

Thus, the insurance industry is highly exposed to climate risk; but it also has the potential to drive climate adaptation, by linking premiums to the provision of adaptive measures. However, no participant in SEQ reported premium rises after the Brisbane floods, or additional requirements for adaptation. However, steep premium rises, likewise without any requirements for adaptation, had occurred in North Queensland, following Cyclone Yasi. Such premium rises might lead to maladaptation, as high prices could result in underinsurance in areas already vulnerable to severe weather events and climate change. Further, the reliance by developers on insurance cover as a risk mitigation strategy for potential severe weather events could be maladaptive, as insurance payouts negate the need for implementing adaptive measures.

Indirect risks, such as more onerous regulation or strengthened building codes, were seen as more important than direct risks, such as cyclones or floods. The regulatory environment was viewed as a major risk, particularly State and Local Government policy. State planning processes were seen as complex, inefficient, inflexible and unable to deal with uncertainty. State Government was criticised because of lack of integration between government agencies, with variable requirements depending on the agency or the assessing officer. 
Local Government was also widely criticised, particularly on delays in approving developments (especially innovative developments), unsophisticated planning staff (particularly in regional LGAs), and inconsistencies between State and Local regulations.

Some legislation, such as the now suspended Coastal Plan, were widely criticised by virtually all participants. Other regulatory measures aimed at forcing developers to adapt, such as planned retreat, were also viewed extremely negatively. Litigation was not seen as a threat to developers; and was more commonly a maladaptive measure, as developers took action against LGAs that tried to implement measures, such as planned retreat. Developers felt that, as long as they adhered to the BCA and all regulatory requirements, they were protected against litigation. Conversely, the BCA was generally viewed positively. Perhaps because of the certainty associated with such codes, participants saw the BCA as having the potential to manage climate change risks. This had already happened in the past, as single climate events, such as Cyclone Tracey, had driven rapid changes to building codes.

The regulatory environment was seen as both a barrier and a driver of adaptation. Participants held diverse views on regulation; some felt that the industry and the market should drive innovation and adaptation to climate change, but others felt that developers would not adapt without "the regulatory stick". For example, some participants supported implementing adaptive measures into developments; i.e. raising floor levels or moving critical infrastructure to higher levels. However, few had actually done so unless required to do so by regulation.

Moreover, the majority wanted any climate change adaptation legislation to be logical and practical, and to be introduced incrementally, with comprehensive consultation with industry. Participants felt that the most onerous issues resulting from regulatory change were increased approval times, government charges, and economic impacts such as lack of market demand, and problems with financing. The GFC had severely impacted the development industry, and this was exacerbated by financiers unwilling to loan money, and excess regulation, which delayed development approvals, and cost money.

The commercial sector also reported strong support for voluntary rating and certification schemes, such as Green Star, NABERS and EnviroDevelopment, with developers often competing with one another over ratings. This was driven by developers' retaining ownership of commercial buildings and saving on operations and maintenance; demands by quality tenants; and government subsidies.

However, there was virtually no demand for sustainable (or "green") properties from any sector of the residential market, except, to a minor degree, from the retirement sector. This lack of demand was largely blamed on the GFC and economic conditions; buyers were more interested in price and immediate tangible benefits (such as larger rooms), and were unwilling to pay for long term intangible benefits. Thus industry selfregulation appears to be a potentially valuable driver for sustainability and increased adaptation to climate change. However, if some way of translating this to the residential market could be found, they could be powerful drivers of adaptive capacity.

The actual adaptive capacity of developers was dynamic and flexible, and a combination of interrelated factors. These factors were not discrete, and any factor could act as a barrier or a driver, depending on the type of climate impact, and a range of places and scenarios. Adaptive capacity could be categorised into participants (i.e. developers); internal characteristics (i.e. attitude and brand); development characteristics (i.e. spatiotemporal scale); governance and regulation (i.e. legislation); and economic and market characteristics (i.e. economic resources and financiers). 
The most important drivers of adaptive capacity were economic and market characteristics, participant characteristics and internal structure, and regulatory characteristics. Market demand was a strong driver of adaptation, but generally only in boom times. Market conditions and the availability of finance largely dictated the type of development, particularly for smaller firms. Banks were unwilling to lend money without a high proportion of pre-sales, and the market was highly price conscious, and not prepared to pay extra for sustainable products.

Larger developers were generally more adaptive, as they were less reliant on bank funding; had a large diversity of product; could afford to employ specialist consultants; were more conscious of leaving an enduring legacy; and tended to produce larger and lengthier developments. On the other hand, some smaller developers were also passionate about producing innovative, quality product, were self-educated, and subscribed to industry self-regulation schemes.

Although respondents considered their knowledge of climate change was good, this was not backed up by evidence. A number of participants were very sceptical about the anthropogenic nature of climate change, and the type, time and scale of possible events. Many associated climate change only with sea level rise, and were unconcerned, as they felt it would impact too far in the future, if at all. Few respondents mentioned risks other than cyclones or flooding, unless prompted.

Given that these determinants of adaptive capacity are so complex, and interrelated, some respondents felt it would be better to view this from a systems perspective, rather than a "tick box" list. For example, a sustainability consultant felt that adaptive capacity changed all the time, a firm could be adaptive one day, and not the next. Another developer told of a change in dam management that inundated part of a previously flood-free site. Therefore, no matter what the adaptive capacity of a given property developer, external issues totally beyond their control could have a negative impact.

\subsection{Knowledge Gaps and Future Research Directions}

A marked dichotomy was apparent between commercial and residential developers, in the incorporation of adaptive measures into buildings, and the emphasis on voluntary sustainability schemes, such as Green Star. Further investigation into methods to encourage residential developers to incorporate climate change adaptation into their developments would likely prove fruitful.

Economic issues, particularly post GFC market demand and stringent lending requirements by banks, were some of the major barriers to a firm's adaptive capacity, particularly smaller firms. The role of finance in development and how it influences development outcomes is poorly dealt with in the scholarly literature. The positive side of this is that there is considerable scope for empirical work to investigate this matter, particularly for more recent development trends, such as the financier's insistence on a high percentage of pre-sales. The short time scale of loans apparently exacerbates maladaptation, as developers consider their exposure to climate risk as very low.

In the short term, it is often not cost-effective to adapt, but in the long term, it is more beneficial. This problem identifies a possible new research agenda; to identify new mechanisms by which the short term incentives to not undertake adaptation and planning responses are reduced in favour of longer run incentives to undertake adaptive challenges. Given the nature of the climate change problem, this is not however, an issue that is unique to the private development sector; it is a challenge that affects almost all sectors in their response to the challenge of climate change.

The urban property development industry comprises the developers themselves; but also a wide range of allied professionals, such as consultants, engineers, subcontractors, architects, solicitors, insurers, financiers, and builders. These other 
industries perform essential supportive and collaborative roles. Participants in this study reported that builders and subcontractors were very conservative, and often blocked adaptive measures on site. Further investigation of potential for the construction industry to be a barrier (or driver) of adaptation, would be useful.

Another important research gap is the role of insurers in climate change adaptation, or maladaptation. Insurers were not seen as posing any problems to SEQ developers; instead many spoke of using insurance as a rationale for not incorporating adaptive measures. One even mentioned not moving infrastructure, because it was likely the next flood would happen when it needed replacing. However, insurers in North Queensland had significantly raised premium, particularly for strata titled units. Unless price rises are coupled with rebates for adaptive measures, premium rises will likely drive consumers or even developers away from properly insuring their property.

Therefore, the insurance industry appears to be promoting maladaptation rather than adaptation. Although lenders insist on insurance, one banker stated that they rarely check whether this has been done. Further, unless insurers place limits on developers, they will continue to use insurance as a backup in case anything happens. Therefore, the insurance sector has a significant potential to increase or decrease adaptive capacity, and needs more research.

Of note, a relatively high proportion of property developers and consultants (particularly planning consultants) appeared to have limited knowledge on climate change, and the potential impacts it may pose to their businesses. This lack of knowledge was particularly relevant to the physical impacts of climate change, such as an increased risk of floods, droughts, and more intense cyclones tracking further south. Many of these were unconcerned about the risk of climate change, because they associated it only with sea level rise, and saw this happening, if at all, many years in the future. Therefore, it would be worthwhile to investigate means by which the different physical aspects of climate change and the risks of these can be better communicated; including more detailed research on the sources of information used by the property development industry.

The study flagged that taking an integrated systems approach to address this issue may be worthwhile. Pragmatically speaking, for policy-makers, this could just mean seeking more holistic solutions, working with industry groups, the community and the development industry. Public participation is fundamental to many planning systems around the world, but in practice is often given lip service, or hijacked by single interest groups.

A systems approach is also relevant when considering communicating and disseminating information on climate change; whether producing awareness raising information for industry members, or distributing data, such as GIS layers, to support development planning. Good decision making, such as where to site development infrastructure, depends on good data. If government agencies are unwilling or unable to release data, this could have long term impacts on the future sustainability of urban areas. A systems approach would also look at data from areas external to the project site. The current Queensland planning and development system is often site-specific, and only investigates potential issues directly related to a site. As was highlighted on $p$ 116, a LGA change of management to an upstream dam had major impacts on a development site further down a river.

\subsection{Limitations}

Researchers have to be mindful of sampling and the extent to which it is representative of the composition and diversity of the industry in a given period. Too narrow a sample can miss important perspectives. Engaging with, and even identifying firms from across the whole spectrum of developer types in the industry, poses a challenge in the context

122 The capacities of private developers in urban climate change adaptation 
of a large, ever-changing, and heterogeneous industry. Further, researchers need to be mindful of the type of player who is interviewed when interpreting the findings of qualitative and interview based research.

All respondents were self-selected; thus it may be argued that they already have some knowledge and interest in climate change adaptation; and at worst, might be biased. Moreover, the initial contacts for the interviews were provided by the UDIA EnviroDevelopment manager, and were likely already sensitised to the issue of climate change and relatively proactive. However, the general climate scepticism expressed by many may indicate a relatively balanced view. Of note, it is impossible to randomly select respondents by snowballing, so this potential bias cannot be overcome.

The original questionnaire survey was distributed via the UDIA email newsletter list, and it was impossible to estimate the exact number of recipients. Therefore, due to low response to this method (one reply), the survey was sent by direct email to developers and consultants with email addresses listed on public websites. As only those respondents with active websites were contacted, these results are likely not indicative of all developers, particularly smaller developers without websites.

Further, all survey questions were optional, and some applied only to specific respondents; and no one respondent completed every question. Therefore, some types of statistical analyses were impossible, and it must be cautioned that all sample sizes were relatively small, particularly when differentiating respondents by category (i.e. type of developer or consultant).

For the three focus groups, it was originally aimed to differentiate the participants by category; i.e. developers, consultants and peak bodies (i.e. UDIA, Australian Green Infrastructure Council, Planning Institute of Australia, State Government). However, due to difficulties with scheduling, the eventual focus groups were more diverse, with representatives from more than one category. The average number of participants was also low, around three participants per focus group.

Of note, the original research proposal aimed to contrast SEQ developers with Victorian (Melbourne) developers. However, it proved extremely difficult and time consuming to contact members of the Victorian development industry. The Queensland Branch of the UDIA was extremely helpful in assisting the research team, however, it was difficult to contact their Victorian counterparts. In the end, this aspect of the research was not conducted. It is not considered a major limitation, as arguably,

Victoria and Queensland have markedly different property development sectors.

Although a more limited study than originally planned was conducted, the work still references the broader context; such as regulations and planning policy in Australia and in other states. Moreover, the literature review preceding each chapter begins with a discussion on general scholarly research specific to that section (i.e. adaptation), prior discussing specific details of the local SEQ context.

Finally, it is important to note that all comments are only the personal opinion of the respondent, and no representations on their veracity and accuracy can be made.

\subsection{Recommendations for policy makers}

The question is thus asked, how can climate adaptation policy improve the development industry's capacity to deliver climate adaptive urban development and, conversely, not impede this capacity or contribute to maladaptation? This is particularly important, given the spatial immobility and path dependence of the built environment.

A number of methods (and agents) exist to enhance (or hinder) the adaptive capacity of the private property development industry. For example, the financial sector can hinder a developer's adaptive capacity by imposing stricter conditions, and shorter time 
frames on loans. Conversely, stricter conditions on finance can enhance the overall adaptive capacity of the broader economy, by avoiding loan defaults and economic decline; and thus giving greater resilience to climate change.

Thus, it is obvious that multiple, often competing goals exist in this space. To this end, policy makers will need to be aware of the various goals, and the trade-offs that might be required. Moreover, it is important to ensure that any decisions made do not lead to future path dependency or maladaptation. This issue is, of course, well recognised by policymakers and other stakeholders, however, is worth restating.

Therefore, Table 9 (below) offers some key findings, recommendations for policy makers, and suggested agents to implement some or a combination of these. These recommendations are not prioritised, either in terms of urgency, or degree of difficulty, and nor have they been subject to any cost benefit analysis. Some may be relatively simple to address, while others may be extremely difficult to achieve. Moreover, these are given as discrete choices, where in reality, they are interconnected, dependent on the cooperation of multiple actors, and the attitudes of individual developers, staff within development firms, and the consultants hired to advise them.

For example, a key finding of this study was that many developers and planning consultants do not feel that climate change poses a serious threat to their business; and associate it only with sea level rise. The recommendation is made for "Improved communication and awareness-raising regarding the risks of climate change, and what it actually is". Producing communications and awareness material is relatively simple; as is distributing this to specific stakeholder groups (such as property developers).

However, even if this leads to an improved knowledge of climate change, this may well not be sufficient to change behaviour, which is far more difficult. Moreover, as was evidenced during this study, even if respondents were well aware of the potential threats from climate change, they were constrained by other circumstances, such as lack of market demand and the low exposure to climate risk due to the relatively short development process.

Nonetheless, to ensure the most cost-effective use of public funds, policy measures should be carefully developed; to strategically target those industry groups that have can have the most impact, as well as those with the greatest capacity to change (Allon \& Sofoulis, 2006; Jorgensen, Graymore, \& O'Toole, 2009; Shearer, 2012). For example, in the context of the development industry, by targeting innovative "champion" developers with wide influence on their peers. One manner in which this may be achieved (and is already being done) is to use the resources of industry bodies, such as the UDIA, to identify and communicate to these individuals.

With reference to Table 9; a possible timetable for implementation may be considered in light of the forecast impacts of climate change. Many of these recommendations (i.e. improved awareness and communication) could be easily carried out in the short to medium term ( 2020). Others (i.e. encouraging demand in the residential sector) may require a combination of circumstances, such as an improved economy, increased political will, government incentives, and a series of major weather events. 
Table 9. Key findings and associated recommendations

\begin{tabular}{|c|c|c|}
\hline Key Finding & Recommendation & Responsible \\
\hline $\begin{array}{l}\text { 1. Developer structure and } \\
\text { development type: } \\
\text { - Larger developers with bigger } \\
\text { projects were more likely to have } \\
\text { greater adaptive capacity } \\
\text { - Consultants were highly } \\
\text { influential and commonly used } \\
\text { - Commercial developers were } \\
\text { actively competing with one } \\
\text { another, and introducing } \\
\text { adaptive and mitigation } \\
\text { measures }\end{array}$ & $\begin{array}{l}\text { - Investigate methods to } \\
\text { encourage similar demand for } \\
\text { green measures by residential } \\
\text { buyers/ residential developers } \\
\text { introduce adaptive measures } \\
\text { - Provide incentives for adaptive } \\
\text { residential developments } \\
\text { Identify and address, if possible, } \\
\text { barriers preventing smaller } \\
\text { developers from implementing } \\
\text { adaptive measures }\end{array}$ & $\begin{array}{l}\text { Govt } \\
\text { UDIA } \\
\text { Developers } \\
\text { Academia }\end{array}$ \\
\hline $\begin{array}{l}\text { 2. Attitude, awareness and } \\
\text { information: } \\
\text { - Most developers and planning } \\
\text { consultants do not feel that } \\
\text { climate change poses a serious } \\
\text { threat to their business. } \\
\text { - Most associate climate change } \\
\text { only with sea level rise. } \\
\text { - GIS data can be expensive, low } \\
\text { quality \& difficult to source }\end{array}$ & $\begin{array}{l}\text { - Improved communication and } \\
\text { awareness raising regarding the } \\
\text { risks of climate change, and what } \\
\text { it actually is. } \\
\text { - Improved access to GIS and } \\
\text { other data on climate change } \\
\text { risks to support development } \\
\text { applications (including } \\
\text { government opening up access } \\
\text { to data) }\end{array}$ & $\begin{array}{l}\text { Media } \\
\text { UDIA } \\
\text { Govt. } \\
\text { Academia }\end{array}$ \\
\hline $\begin{array}{l}\text { 3. Economics and market: } \\
\text { - Post GFC economic factors/ } \\
\text { bank lending conditions are } \\
\text { major barriers to adaptive } \\
\text { capacity } \\
\text { - The insurance industry is } \\
\text { potentially a driver of adaptation } \\
\text { - A general lack of demand and } \\
\text { unwillingness to pay for "greener" } \\
\text { products in the residential market }\end{array}$ & $\begin{array}{l}\text { - Improve awareness of climate } \\
\text { change and adaptation for major } \\
\text { development financiers (and } \\
\text { insurers) } \\
\text { - } \quad \text { Reduced insurance premiums/ } \\
\text { interest rates for providing } \\
\text { adaptive measures in } \\
\text { developments. } \\
\text { - Cost effective adaptive measures } \\
\text { "sold" in developments } \\
\text { - Awareness raising re long term } \\
\text { savings for green measures }\end{array}$ & $\begin{array}{l}\text { Banks } \\
\text { Insurers } \\
\text { Developers } \\
\text { Media } \\
\text { UDIA }\end{array}$ \\
\hline $\begin{array}{l}\text { 4. Governance and regulation: } \\
\text { - State and LGA regulation and } \\
\text { planning processes are viewed } \\
\text { as complex, inflexible and } \\
\text { inconsistent. } \\
\text { - LGA staff seen as } \\
\text { unsophisticated, and unable to } \\
\text { deal with uncertainty (not in the } \\
\text { Planning Codes) } \\
\text { BCA seen as a major vehicle for } \\
\text { adaptation, with rapid results } \\
\text { after climate impact (also } \\
\text { supported by industry) }\end{array}$ & $\begin{array}{l}\text { Increased and continued } \\
\text { cooperation between all levels of } \\
\text { Govt. and developers; } \\
\text { consultants and industry bodies. } \\
\text { State Government and industry } \\
\text { bodies collaborate to increase } \\
\text { LGA awareness, particularly for } \\
\text { smaller councils. } \\
\text { Emphasise BCA/ Building Codes } \\
\text { Board, as a method by which } \\
\text { adaptive measures can be } \\
\text { legislated, especially given their } \\
\text { general acceptance by } \\
\text { developers. }\end{array}$ & $\begin{array}{l}\text { Govt. } \\
\text { Developers } \\
\text { Consultants } \\
\text { UDIA } \\
\text { ABCB }\end{array}$ \\
\hline
\end{tabular}




\section{REFERENCES}

ABC News (21 December 2011). Australian Broadcasting Corporation. Retrieved from http://www.abc.net.au/news/2011-12-21/insurance-losses-amount-to-billions/3741932

ABS. (2009) 1386.0 - What's New in Regional Statistics, Jun 2009.

Adams, D., Croudace, R., \& Tiesdell, S. (2012). Exploring the 'notional property developer' as a policy construct. Urban Studies, Published Online First 5 January, 20 pages.

Adams, D., Dunse, N., \& White, M. (2005). Conceptualising state-market relations in land and property: the growth of Institutionalism - extension or challenge to mainstream economics. In D. Adams (Ed.), Planning, Public Policy \& Property Markets (pp. 37-55). Oxford: Blackwell.

Adger, W. N., Agrawala, S., Mirza, M. M. Q., Conde, C., O'Brien, K., Pulhin, J., Takahashi, K. (2007). 2007: Assessment of adaptation practices, options, constraints and capacity. Climate Change 2007: Impacts, Adaptation and Vulnerability. Contribution of Working Group II to the Fourth Assessment Report of the Intergovernmental Panel on Climate Change (pp. 717-743). Cambridge: Cambridge University Press.

Allen, D., \& Faff, R. (2012). The Global Financial Crisis: some attributes and responses. Accounting and Finance, 52(1).

Allon, F., \& Sofoulis, Z. (2006). Everyday Water: Cultures in transition. Australian Geographer, 37(1), 45-55.

Anderson, K., \& Bows, A. (2011). Beyond 'dangerous' climate change: emission scenarios for a new world. Philos Transact A Math Phys Eng Sci, 369(1934), 20-44.

Ashe Morgan Winthrop. (2011). Property Development Finance Post GFC, from http://www.qld.api.org.au/assets/media library/000/000/206/original.pdf?1314684262

Australia Green Infrastructure Council (AGIC). (2010). AGIC guidelines for Climate Change Adaptation.

Australian Building Codes Board. (11 October 2011). The Building Code of Australia, from http://www.abcb.gov.au/about-the-national-construction-code/the-building-codeof-australia

Australian Building Codes Board. (2012). Regulation Impact Statement for Final Decision: Proposal to revise the National Construction Code requirements for construction in cyclone regions. In F. G. o. Australia (Ed.). Canberra: Australian Building Codes Board.

Australian Government Actuary. (2012). Report On Investigation Into Strata Title Insurance Price Rises In North Queensland. Canberra, ACT: Federal Government of Australia.

Australian Government Productivity Commission. (2011). Barriers to Effective Climate Change Adaptation. Melbourne, Victoria: Australian Government

Australian Government. (2011). Securing a Clean Energy Future: The Australian

Government's Climate Change Plan. Retrieved from

http://www.cleanenergyfuture.gov.au/wp-content/uploads/2011/07/Consolidated-

Final.pdf.

Ball, M. (1998). Institutions in British property research: a review. Urban Studies, 35(9), 1501-1517. 
Bambrick, H. J., Capon, A. G., Barnett, G. B., Beaty, R. M., \& Burton, A. J. (2011). Climate Change and Health in the Urban Environment: Adaptation Opportunities in Australian Cities. Asia-Pacific Journal of Public Health, Supplement to 23(2).

Barnett, J., \& O'Neill, S. (2010). Maladaptation. Global Environmental Change, 20, 211-213.

Bengtsson, J. (2008a). Adaptation to Climate Change- Sea level rise and flooding. Retrieved from yourdevelopment.org/factsheet/view/id/62

Bengtsson, J. (2008b). Possible effects on buildings through climate change. Retrieved from http://yourdevelopment.org/factsheet/view/id/65

Berkhout, F., Hertin, J., \& Gann, D. M. (2006). Learning to adapt: organisational adaptation to climate change impacts. Climatic Change, 78(1), 135-156.

Bindoff, N. L., Willebrand, J., Artale, V., Cazenave, A., Gregory, J. M., Gulev, S., \& Unnikrishnan, A. S. (2007). Observations: oceanic climate change and sea level. In Solomons et al (Eds.), Climate Change 2007: The Physical Science Basis. Contribution of Working Group 1 to the Forth Assessment Report of the Intergovernmental Panel on Climate Change (pp. 385-432). Cambridge and New York: Intergovernmental Panel on Climate Change.

Birrell, G., \& Gao, S. (1997). The UK property development process: its phases ad their degree of importance to profitability. Paper presented at the RICS Cutting Edge Conference, Dublin.

http://www.rics.org/site/download feed.aspx?filelD=2101\&fileExtension=PDF

Bosello, F., Carraro, C., \& De Cian, E. (2009). An Analysis of Adaptation as a Response to Climate Change Copenhagen

Bouwer, L. M. (2011). Have disaster Losses Increased due to anthropogenic climate change? American Meteorological Society.

Braganza, K., \& Church, J. A. (2011). Observations of Global and Australian Climate. In H. Cleugh, et al (Eds.), Climate Change; Science and Solutions for Australia (pp. 114). Victoria: CSIRO.

Briggs, P., Taberner, J., Voros, M., \& Bilski, A. (2010). Climate Change litigation to flood planning and development in coastal areas. Retrieved from http://www.freehills.com/5789.aspx

Bryant, L. (2012). An assessment of development funding for new housing post GFC in Queensland, Australia. International Journal of Housing Markets and Analysis, 5(2), 118 - 133.

Bryson, J. R., \& Lombardi, R. (2009). Balancing product and process sustainability against business profitability: Sustainability as a competitive strategy in the property development process. Business Strategy and the Environment 18, 97-107.

Bureau of Meteorology (BOM). (2011). Tropical Cyclone Trends, from http://www.bom.gov.au/cyclone/climatology/trends.shtml

Burton, I. (1996). The growth of adaptation capacity: practice and policy. Adapting to climate change: an international perspective, 55-67.

Bussey, M., R. W. , Carter, N., Keys, J., Carter, R., Mangoyana, J., Matthews, D., \& Smith, T. F. (2011). Framing adaptive capacity through a history-futures lens: lessons from the South East Queensland Climate Adaptation Research Initiative Futures, 44(4), 385-397 
Chen, K., \& McAneney, J. (2010). Bushfire Penetration into Urban Areas in Australia: a Spatial Analysis, from http://www.bushfirecrc.com/managed/resource/bushfire-

penetration-urban-areas.pdf

Climate Risk (2008). From Risk to Advantage: General Insurers as Key Agents for Climate Change Adaptation.

Coiacetto, E. (2001). Diversity in Real Estate Developer Behaviour: A case for research. Urban Policy and Research, 19(1), 43-59.

Coiacetto, E. (2006). Real estate development industry structure: consequences for urban planning and development. Planning Practice and Research, 21(4), 423-441.

Coiacetto, E. (2007). Residential sub-market targeting by developers in Brisbane. Urban Policy and Research, 25(2), 257-274.

Coiacetto, E. (2009). Industry structure in real estate development: Is city building competitive? Urban Policy and Research, 27(2), 117-135.

Coiacetto, E. (2012). A new way for built environment professionals to understand and think about land development: Understanding Land Development: A project based approach. Collingwood, Victoria: CSIRO Publishing.

Crichton, D. (2003). Insurance implications of climate change. Paper presented at the Managing risk and liability in a changing climate, London.

CSIRO. (2011). Climate Change Science and Solutions for Australia. Collingwood, Victoria.

Decker, S. O. (2004). Corporate social responsibility and structural change in financial services. Managerial Accounting Journal 19(6), 712- 728.

Della-Marta, P., Collins, D., \& Braganza, K. (2003). Updating Australia's high-quality annual temperature database. Australian Meteorological Magazine, 53, 57-67.

Deloitte Touche Tohmatsu. (2011). Australia's carbon pricing mechanism- Key issues for business, from http://www. deloitte.com/assets/Dcom-

Australia/Local\%20Assets/Documents/Services/Climate\%20change\%20and\%20sustai nability/Deloitte carbon pricing mechanism\%20.pdf

Department of Climate Change and Energy Efficiency (DCCEE). (2011). Climate Change Risks to Coastal Buildings and Infrastructure: A supplement to the First Pass National Assessment

Department of Climate Change. (2009). Climate Change Risks to Australia's Coast:

The First Pass National Assessment from

http://www.climatechange.gov.au/ /media/publications/coastline/cc-risks-full-report.pdf

Department of Climate Change. (2011). Climate Change: Counties Acting Now.

Department of Climate Change and Energy Efficiency (DCCEE),. (2011). Climate Change Risks to Coastal Buildings and Infrastructure: A supplement to the First Pass National Assessment

Dodson, J., Coiacetto, E., \& Banhalmi-Zakar, Z. (2011). Developing adaptively: The role and capacities of private sector developers and financing in urban climate change adaptation. Griffith University.

Durack, P., \& Wijjfels, S. (2010). 50 years trends in global ocean salinities and their relationship to broad-scale warming. Journal of Climate, 23, 4342-4362.

Engle, N. L. (2011). Adaptive capacity and its assessment. Global Environmental Change, 21, 647-756.

128 The capacities of private developers in urban climate change adaptation 
European Investment Bank (EIB), (2012). Climate Action Retrieved March 2012, 2012, from http://www.eib.org/projects/topics/environment/climate-action/index.htm

Fitzgibbon, T. (2010). Legal Consequences of Climate Change; from

http://www.propertyoz.com.au/Article/NewsDetail.aspx?p=56\&mid=1653

Francis, J. J., Eccles, M. P., Johnston, M., Walker, A. E., Grimshaw, J. M., Foy, R., \& Bonetti, D. (2004). Constructing questionnaires based on the theory of planned behaviour. A manual for health services researchers.

Freehills. (2010, 12 February 2010). Climate change litigation to flood planning and development in coastal areas, from http://www.freehills.com/5789.aspx

Garnaut, R. (2011). The Garnaut Review 2011 Australia in the Global Response to Climate Change. Cambridge: Cambridge University Press.

Geoscience Australia (GA) (2001). What is a Flood, 2012, from

http://www.ga.gov.au/hazards/flood/flood-basics/what.html

Glaser, B. G., \& Strauss, A. (1967). The Discovery of Grounded Theory: Strategies for Qualitative Research. Chicago, IL: Aldine Publishing Co.

Gleeson, B. D., J., \& Spiller, M. (2010). Metropolitan governance for the Australian city: The case for reform.: Urban Research Program, Griffith University.

Gore, T., \& Nicholson, D. (1991). Models of Land Development Process: A Critical Review. Environment and Planning 23, 705-730.

Government News. (2011, 31 August 2011). UDIA calls for carbon tax certainty, Government News. Retrieved from

http://www.governmentnews.com.au/2011/08/31/article/UDIA-calls-for-carbon-taxcertainty/RQUUJPIGNN.html

Green Cross Australia. (2011). Harden Up: Protecting Queensland. Severe weather archive.

Guzy, G. S. (2007). A Change in the Weather: New Climate Change Challenges Increase the Potential for Litigation and Regulation. Viewpoint, from http://www.c2es.org/

Hales, A. C. (2010). Resilient Buildings and Resilient Property Developers: A case study of Three Property Development Companies University of Birmingham.

Hall, T. (2009). The Death of the Australian Backyard - A Lesson For Canberra. Paper presented at the Sustainable future project: Bush Capital - Our city in the landscape, Canberra, ACT.

http://www.actpla.act.gov.au/topics/significant projects/planning studies/sustainable f uture/workshop five/presenters menu

Hansen, J. (2008). Tipping point: Perspective of a climatologist. In E. Fearn (Ed.), State of the Wild 2008-2009: A Global Portrait of Wildlife, Wildlands, and Oceans (pp. 6-15):

Wildlife Conservation Society/lsland Press.

Harper, I., \& Thomas, M. (2009). Making Sense of the GFC - Where did it Come from and what do we do Now? Economic Papers: A journal of applied economics and policy, 28(3).

Hastings, A., \& Wysham, D. B. (2010). Regime shifts in ecological systems can occur with no warning. Ecology Letters, 13(4), 464-472.

Hay, C. (1995). Structure and Agency. In A. Marsh \& G. Stoker (Eds.), Theory and Methods in Political Science. Basingstoke: Macmillan Jessop, B. (1996). Interpretative sociology and the dialectic of structure and agency. Theory Culture and Society 13, 119-128.

The capacities of private developers in urban climate change adaptation 129 
Healey, P. (1991). Models of the development process: A review. Journal of Property Research, 8(3), 219-238.

Hennessy, K., Lucas, C., Nicholls, N., Bathols, J., Suppiah, R., \& Ricketts, J. (2005). Climate Change Impacts on Fire-Weather in South-East Australia.

Hoffmann, M., Worthington, A., \& Higgs, H. (2006). Urban water demand with fixed volumetric charging in a large municipality: The case of Brisbane, Australia. Australian Journal of Agricultural and Resource Economics, 50(3), 347-359.

Hughes, G., Lewins, A., \& Silver, C. (2010, 11 February 2010). Analysing Open-Ended Survey Question Data In CAQDAS Packages: Data Preparation For Nvivo (V7 And V8), 2012, from

http://www2.surrey.ac.uk/sociology/research/researchcentres/caqdas/support/analysing survey/analysing openended survey question data in caqdas packages data prep aration for nvivo v7 v8.htm

Institute of Actuaries of Australia (IAA), (2011). Submission to the Garnaut Climate Change Review - Update 2011, from

http://www.pc.gov.au/ data/assets/pdf file/0017/114533/sub043.pdf

Insurance Australia group (IAG). (2002). The Impacts of Climate Change on Insurance Against Catastrophes from

http://stephenschneider.stanford.edu/Publications/PDF Papers/IAG-

Climate Change Paper.pdf

Insurance Council of Australia (ICA). (2011). Disaster Statistics, from

http://www.insurancecouncil.com.au/industry-statistics-data/disaster-statistics

Insurance Council of Australia. (2012). House Of Representatives Standing Committee On Social Policy And Legal Affairs' Inquiry Into Residential Strata Insurance -, from http://www.insurancecouncil.com.au/assets/files/committee $\% 20$ secretary $\% 20$ inquiry $\% 2$ 0into\%20residential\%20strata\%20insurance.pdf ;

http://www.insurancecouncil.com.au/issues-submissions/issues/residential-stratainsurance

Intergovernmental Panel on Climate Change (IPCC), (2011). Summary for Policymakers. In: Intergovernmental Panel on Climate Change Special Report on Managing the Risks of Extreme Events and Disasters to Advance Climate Change Adaptation, from http://www.ipcc.ch/pdf/specialreports/srren/SRREN FD SPM final.pdf

IPCC. (2007). Climate Change 2007 Synthesis Report.

http://www.ipcc.ch/publications and data/publications ipcc fourth assessment report synthesis report.htm

IPCC. (2011). Summary for Policymakers. In: Intergovernmental Panel on Climate Change Special Report on Managing the Risks of Extreme Events and Disasters to Advance Climate Change Adaptation, from http://www.ipcc.ch/pdf/specialreports/srren/SRREN FD SPM final.pdf

Jorgensen, B., Graymore, M., \& O'Toole, K. (2009). Household water use behavior: An integrated model. Journal of Environmental Management, 91(1), 227-236.

Kiehl, J. T., \& Trenberth, K. E. (1997). Earth's Annual Global Mean Energy Budget. Bull. Amer. Meteor. Soc., 78, 197-208.

Krosnick, J. A., \& Presser, S. (2010). Handbook of Survey Research, Second Edition (Vol. Second Edition): Emerald Group Publishing Limited.

Li, G. (2009). (Mal)adapting to tropical cyclone risk: the case of 'Tempestuous Tracy'. The Australian Journal of Emergency Management 24(3), 44-51.

130 The capacities of private developers in urban climate change adaptation 
Local Government Association Queensland (LGAQ). (2012). Half-baked planning policies no good for state's economic health, from

http://www.lgaq.asn.au/web/guest/news/-/journal content/56/10136/1651511

Logan, J. (1993). Cycle and Trends in the Globalization of Real Estate. In P. L. Knox (Ed.), The Restless Urban Landscape (pp. 35-54). Englewood Cliffs, New Jersey: Prentice Hall.

McWilliams, A., D. S. Siegel, et al. (2006). Guest Editors' Introduction: Corporate Social Responsibility: Strategic Implications. Journal of Management Studies . 43(1), 1-18.

Meinshausen, M., Meinshausen, N., Hare, W., Raper, S. C., Frieler, K., Knutti, R., \& Allen, M. R. (2009). Greenhouse-gas emission targets for limiting global warming to 2 degrees C. [Research Support, Non-U.S. Gov't]. Nature, 458(7242), 1158-1162

Melia, S. P. G., \& Barton, H. (2011). The paradox of intensification. Transport Policy 18(1), 46 - 52.

Miles, M., Berens, G., \& Eweiss, M. (2000). Real Estate Development (3rd ed. ed.). Washington DC. Urban and Land Institute.

Mills, E. (2009). From Risk to Opportunity: Insurer Responses to Climate Change (pp. 100 ): Ceres.

Mills, E., Lecomte, E., \& Peara, A. (2001 ). U.S. Insurance Industry Perspectives on Global Climate Change.

Munich RE. (2009). Climate Change and Impacts, from

http://www.munichre.com/app pages/www/@res/pdf/media relations/press releases/2 009/2009 1126 app1 en.pdf

Nalewaik, A., \& Venters, V. (2009). Cost Benefits of Building Green. Cost Engineering, 51(2), 28-34.

National Greenhouse and Energy Reporting Act 2007, 175 (2007).

National Sea Change Task Force (NSCTF). (2011). Submission to the Productivity Commission on Barriers to Effective Climate Change Adaptation: NSCT.

New, M., Liverman, D., Schroder, H., \& Anderson, K. (2011). Four degrees and beyond: the potential for a global temperature increase of four degrees and its implications. Philosophical Transactions of the Royal Society, 369, 6-19.

Nicholls, R. J., Hanson, S., Herweijer, C., Patmore, N., Hallegatte, S., Corfee-Morlot, J., (2007). Ranking of the world's cities most exposed to coastal flooding today and in the future: OECD.

NSW Office of Environment and Heritage. (2012). NABERS: National Australian Built Environment Rating System, 2012, from

http://www.nabers.gov.au/public/WebPages/Home.aspx

Office of Urban Management. (2009). South East Queensland Regional Plan: 2005 2026.

Owens, T. (2012). Defining the Constant Comparative Method, 2012, from http://www.qdatraining.eu/content/defining-constant-comparative-method

Perinotto, T. (2012, 19 September 2012 ). NSW coastal planning in storm of confusion, from http://www.thefifthestate.com.au/archives/38793/

Prakash, A. (2001). Why do firms adopt 'beyond-compliance' environmental policies. Business Strategy and the Environment, 10, 286-299.

Property Council of Australia (PCA). (2012). State Planning Policies create uncertainty for industry, from http://www.propertyoz.com.au/Article/Resource.aspx?media=1932

The capacities of private developers in urban climate change adaptation 131 
PwC. (2011). Economic Impact of Queensland's Natural Disasters. http://pandora.nla.gov.au/col/10701\#11201

QSR International. (2012). Nvivo, 2012, from http://www.qsrinternational.com/products nvivo.aspx

Ratcliffe, J., Stubbs, M., \& Keeping, M. (2009). Urban Planning and Real Estate Development (3rd ed.). London: Routledge.

Sanderson, M. G., Hemming, D. L., \& Betts, R. A. (2011). Regional temperature and precipitation changes under high-end ( $>/=4$ degrees $C$ ) global warming. Philos Transact A Math Phys Eng Sci, 369(1934), 85-98.

Shearer, H. (2012). Using Geographic Information Systems to Explore the Determinants of Household Water Consumption and Response to the Queensland Government Demand-Side Policy Measures imposed during the drought of 2006-2008 PhD, Griffith, Unpublished PhD Thesis.

Smit, B., \& Pilifosova, O. (2003). Adaptation to climate change in the context of sustainable development and equity. In J. B. Smit, R. J. T. Klein \& S. Hug (Eds.), Climate Change Adaptive Capacity and Development London: Imperial College Press.

Smit, B., \& Wandel, J. (2006). Adaptation, adaptive capacity and vulnerability. Global Environmental Change, 16, 282-292.

Smit, B., Burton, I., Klein, R. J. T., \& Wandel, J. (2000). An anatomy of adaptation to climate change and variability. Climatic Change, 45, 223-251.

Smith, T. F., Brooke, C., Measham, T., Preston, B., Gorddard, R., Withycombe, G., \& Morrison, C. (2008). Case Studies of Adaptive Capacity: Systems Approach to Regional Climate Change Adaptation Strategies (Report prepared for the Sydney Coastal Councils Group, Sydney Australia). Canberra, ACT: CSIRO Sustainable Ecosystems

Snow, M., \& Prasad, D. (2011). Climate change Adaptation for Building Designers: An Introduction.

Sovacool, B. K. (2011). Hard and soft paths for climate change adaptation. Climate Policy, 11(4), 1177-1183.

Stafford Smith, M., Horrocks, L., Harvey, A., \& Hamilton, C. (2011). Rethinking adaptation for a 4 degrees C world. Philos Transact A Math Phys Eng Sci, 369(1934), 196-216.

Stewart, M. G., \& Wang, X. (2011). Risk Assessment of Climate Adaptation Strategies for Extreme Wind Events in Queensland, from

http://www.csiro.au/resources/ /media/CSIROau/Flagships/Climate\%20Adaptation/Extr emeWind CAF pdf\%20Standard.pdf

Studdert, J. (2012, Oct 27, 2012). Holiday homes can be bargain buys, The Australian.

Syms, P. (2002). Land Development and Design: Blackwell Publishing.

Taygfeld, P., Coiacetto, E., Shearer, H., Dodson, J., \& Banhalmi-Zakar, Z. (2012). Background Paper: Developing Adaptively: The role and capacities of private sector developers and financing in urban climate change adaptation.

Taylor, B. M., Harman, B. P., Heyenga, S., \& McAllister, R. J. (2012). Property Developers and Urban Adaptation: Conceptual and Empirical Perspectives on Governance. Urban Policy and Research, 30(1), 5-24.

The State of Queensland Department of Environment and Resource Management (DERM). (2012a). Queensland Coastal Plan: Coastal Hazard Guideline. Retrieved from http://www.derm.qld.gov.au/coastalplan/pdf/hazards-guideline.pdf .

132 The capacities of private developers in urban climate change adaptation 
The State of Queensland. (2012b). Queensland Coastal Plan. Retrieved from http://www.derm.qld.gov.au/coastalplan/pdf/qcp-web.pdf .

Thompson, T. (2013). Skyrocketing insurance premiums hit Queensland flood victims two years on Retrieved January 7 2013, 2013, from http://www.couriermail.com.au/news/queensland/skyrocketing-insurance-premiums-hitqueensland-flood-victims-and-others/story-e6freoof-1226548519016

Tiesdell, S., \& Allmendinger, P. (2005). Planning Tools and Markets: Towards an extended conceptualisation. In D. Adams, C. Watkins \& M. White (Eds.), Planning, Public Policy \& Property Markets (pp. 56-76). Oxford: Blackwell Publishing.

Trumbo, C. W., \& O'Keefe, G. J. (2005). Intention to conserve water: Environmental values, reasoned action, and information effects across time. Society and Natural Resources, 18(6), 573-585.

United Nations Development Program (UNDP). (2012). Climate Change Retrieved March 2012, from http://www.undp.org/climatechange/adapt/definitions.html\#21

Urban Development Institute of Australia (UDIA), (2011). About Enviro-Development, from http://www.envirodevelopment.com.au/01 cms/details.asp?ID=1

Urban Development Institute of Australia (UDIA) South Australia. (2011). Climate Change Mitigation and Adaptation for the Urban Development Industry. Retrieved from http://www.udiasa.com.au/uploads/UDIA\%20Climate\%20Change \%20briefing\%20paper \%20-\%20final\%20June\%202011\%20 2 .pdf

Urban Development Institute of Australia (UDIA). (2008). Climate Change Discussion Paper and Draft Policy Position, from http://www.udia-

nsw.com.au/resource/UDIA\%20NSW\%20Climate\%20Change\%20Discussion\%20Pape r.pdf

Vasey-Ellis, N. (2011). Planning for Climate Change in Coastal Victoria. Urban Policy and Research, 27(2), 157-169.

Victorian Bushfire Royal Commission (VBRC). (2010). 2009 Victorian Bushfire Royal Commission - Final report.

Walsh, E. (2002). Dealing with Data: Using NVivo in the Qualitative Data Analysis Process. Forum: Qualitative Social Research, Volume 3, (No. 2).

Wang, X., Stafford Smith, M., McAllister, R., Leitch, C. J., McFallan, S., \& Meharg, S. (2011). Coastal inundation under climate change: a case study in South East Queensland. Retrieved from http://www.csiro.au/Organisation-

Structure/Flagships/Climate-Adaptation-

Flagship/ /media/CSIROau/Flagships/Climate\%20Adaptation/WorkingPaper6 CAF pd f\%20Standard.pdf

Ward, R. E. T., Herweijer, C., Patmore, N., \& Muir-Wood, R. (2008). The role of Insurers in Promoting Adaptation to the Impacts of Climate Change. The Geneva Papers 33, 133-139.

Westpac. (2009). Westpac Climate Change Position Statement : Financing the Transition to a Low Carbon Economy, from http://www.westpac.com.au/docs/pdf/aw/Transit to low carbon econo1.pdf

Whitehead, J. C. (1987). Decision making in the property development industry during a business cycle, The University of British Columbia, Unpublished PhD Thesis.

Wiltshier, F. (2011). Researching with Nvivo 8. Forum: Qualitative Social Research, Volume 12(No. 1). 
World Bank. (2012). Adaptation Guidance Notes - Key Words and Definitions, from http://climatechange.worldbank.org/climatechange/content/adaptation-guidance-noteskey-words-and-definitions

World Bank. (2012b). Turn Down the Heat: Why a 4 Degrees Centrigrade Warmer World Must be Avoided: Potsdam Institute for Climate Impact Research and Climate Analytics.

Zhang, Y., \& Wildemuth, B. M. (2009). Qualitative Analysis of Content. In B. Wildemuth (Ed.), Applications of Social Research Methods to Questions in Information and Library Science (pp. pp.308-319). Westport, CT: Libraries Unlimited 


\title{
9. APPENDICES
}

\subsection{Information Sheet and Consent Form for Interview and Focus Group Participants 16}

\section{Developing Adaptively: The role and capacities of private sector developers and financing in urban climate change adaptation}

\author{
INFORMATION SHEET \\ Who is conducting the \\ research
}

\author{
Associate Professor Jago Dodson; Dr Eddo \\ Coiacetto; Ms Zsuzsa Banhalmi-Zakar; Ms Pazit \\ Taygfeld; and Ms Heather Shearer. \\ Urban Research Program, Griffith University \\ Contact: Heather Shearer \\ Telephone: 0407338942 \\ Email: h.shearer@griffith.edu.au
}

\section{Why is the research being conducted?}

The Urban Research Program of Griffith University (with funding and in-kind support from the National Climate Change Adaptation Research Facility (NCCARF), and the Urban Development Institute of Australia (UDIA)), are undertaking a research project to investigate the capacity of the private urban development sector to adapt to climate change.

The outputs of the research will also be used to produce a final project report, and 3 peer reviewed academic publications.

\section{What you will be asked to do}

You will be asked to participate in a one hour semi-structured interview.

\section{The basis by which participants will be selected or screened}

Participants will be members of the urban development industry sector in SEQ and Melbourne; and either developers or developer peak body representatives.

Selection of participants has been undertaken in consultation with the Queensland division of the Urban Development Institute of Australia (UDIA). Participants in the interview process have been identified (with their consent) by the UDIA.

\footnotetext{
${ }^{16}$ Note: Identical for focus group participants, save for replacing interview with focus group, and one hour with one and a half hours.
} 


\section{The expected benefits of the research}

By understanding the capacity of the sector to improve its climate adaptation responses, the project will advance the national objective of ensuring Australian cities can weather the effects of climate change.

\section{Risks to you}

We do not anticipate any risks to you as a result of your participating in this interview process.

\section{Your confidentiality}

Your responses are confidential, and can only be accessed by the researchers. You are not required to give your name or that of your organisation. It may be possible to infer the identity of some organisations because of information collected, but not the identity of the actual person completing the survey. No identifying information will be available to anyone outside the research team, who are bound by the Griffith University Ethics policy.

All material collected will be stored securely in the premises of the Urban Research Program, Nathan Campus, Griffith University. Interviews will be audio-taped; only the research team will have access to this tape; and audio will be erased following transcription.

The results of the research may be used in academic publications and conferences by the researchers, and reports to Industry Partners. No identifying details of the participants or their present or previous employers will be disclosed in any publications.

\section{Your participation is voluntary}

Participation is voluntary and you are free to withdraw from the research at any time.

\section{Questions / further information}

Any questions or requests for further information, please contact Heather Shearer on 0407 338942 or h.shearer@griffith.edu.au.

\section{The ethical conduct of this research}

This survey has been approved by the Griffith University Research Ethics Committee: Approval Number; ENV/54/11/HREC. Griffith University conducts research in accordance with the National Statement on Ethical Conduct in Human Research (2007). If you have any concerns or complaints about the ethical conduct of the research project they should contact the Manager, Research Ethics on 37355585 or research-ethics@griffith.edu.au

\section{Feedback to you}

The project findings and reporting will be accessed by users in three key ways: the Project website; Project bulletins; and Hard copy project reports.

\section{Privacy Statement}

For further information consult the University's Privacy Plan at: http://www.gu.edu.au/ua/aa/vc/pp or telephone (07) 38755585. 


\section{Developing Adaptively: The role and capacities of private sector developers and financing in urban climate change adaptation}

\section{CONSENT FORM}

Research

Team
Jago Dodson; Eddo Coiacetto; Heather Shearer; Pazit Taygfeld; Zsuzsa Banhalmi-Zakar

Urban Research Program, Griffith University

Contact: Heather Shearer

Telephone: 0407338942

Email: h.shearer@griffith.edu.au

By signing below, I confirm that I have read and understood the information package and in particular have noted that:

- I understand that my involvement in this research will include an interview of approximately one hour's duration;

- I understand that my interview will be audio-taped; and

- I understand that only the research team will have access to this tape; and

- I understand that the audiotape will be erased following transcription.

- I have had any questions answered to my satisfaction;

- I understand the risks involved;

- I understand that there will be no direct benefit to me from my participation in this research;

- I understand that my participation in this research is voluntary;

- I understand that if I have any additional questions I can contact the research team;

- I understand that I am free to withdraw at any time, without comment or penalty;

- I understand that I can contact the Manager, Research Ethics, at Griffith University Human Research Ethics Committee on 37355585 (or research-ethics@griffith.edu.au) if I have any concerns about the ethical conduct of the project; and

- I agree to participate in the project.

\begin{tabular}{|l|l|}
\hline Name & \\
\hline Signature & \\
\hline Date & \\
\hline
\end{tabular}




\subsection{Sample Interview Schedule}

\section{Context setting and warm up}

Introduce myself (Senior Research Assistant, background in Environmental Planning with main field of expertise and interests...)

Introduce the research: we are trying to understand how climate change adaptation fits inside the world of the private property development sector and the specific company/firm that you are employed with.

The aim of this interview is to discuss your perspective on what you think are some of the challenges (physical / regulatory) that climate change presents to the development sector and your firm.

Before we start, we need to briefly discuss some ethical issues/housekeeping matters:

- The content of this interview is completely confidential and no identifiable information will be used at any time.

- We would like top record this interview. The reason for this is to give us an opportunity to transcribe and review the material after the interview.

- If you agree to these, can you please sign this consent form?

- Can you please tell us a little bit about yourself and your firm? (e.g., what is your role? how did your firm and yourself got into development?)

- What does your firm do?

$>$ What kind of development does it do and what markets and submarkets is it in?

$>$ Does it only do development? What other activities is your firm involved in (e.g. timber, banking, property investment, mining, etc.)

$>$ Where and how does development fits into your firm's larger operation? (e.g. a small high risk side activity)

$>$ What is the geographical scope of your firm's development operations? What sort of development do you do in SEQ?

$>$ Can you tell me about other developers in SEQ that do different types of development from you? Does what they and you do change over time (e.g. boom bust or place?)

- How is your firm organised (its form- e.g. 2-3 people doing everything?, external consultants?, etc.) can you tell me how this may be different to other developers operating in SEQ? Does it make a difference to the process of development and its outcomes? What are the advantages?

- I would like to hear what do you think are the challenges and risks for development that concern you (e.g. market, GFC, getting finance, planning rules, competition)

- Can you tell me how the funding works for your development/ how that might be different for other developers? Can you give me an idea of how it effects what you can and can't do?

\section{Climate Change}

One issue that we think might have a big impact on development is the big questions of climate change. How it could affect development is not clear- and we are interested in exploring that with you. We are also interested to know how you think developers could get ready for it and what does it mean for the industry. We believe that climate change could have direct impacts on development (e.g. from severe weather events and sea level rise) but also indirect affects thought new rules and regulations, through markets (changing consumer demand, through lending and borrowing, etc.) 
1. Have you given much thought as to how climate change might mean for your business? What it might mean for other developers?

$>$ What do you think about this matter and how it might affect developers?

We have been reading and thinking about this and these are some thoughts we have come up with for what it might mean for development and developers. Can we go over these and get your perspective on them?

We are interested in considering:

$\checkmark$ your and other firm's exposure to these risks

$\checkmark$ other types of risks in the future?

$\checkmark$ How these risks might be managed?

$\checkmark$ What could make it easier to manage such risks?

(a) Site and location risks

- Some sites are more at risk than others- which are at risk and why? E.g. coastal. What sites might open up?

- -specific requirements for development in high risk areas? (different building materials, design requirements?)

- - funding and insurance issues?

(b) Lending and borrowing risks

- Are some projects hard to get finds for?

- Some types of funding might mean that developers won't get money for climate risky projects but they might be able to get other types of funds, e.g. securities, etc.

(c) Regulatory risks

- Planning rules (e.g. coastal plan)

- Infrastructure charges- increased costs perhaps uncertainty about charges

- Changing building codes

- Carbon Tax?

- Building codes?

(d) Market risks

- Do you think some types of sub-markets going to be more sensitive to climate risk (residential, industrial?)

- Do you think that some sub markets groups may be underserviced by the development industry?

- Do you consider how climate change policies and changing insurance practices could affect your target markets and yourself?

2. How to you think that issue of climate change should be handled by (a) developers or (b) regulators and government?

3. When you hear the term "climate change adaptation" what comes to your mind?

4. Do you have any other matters you would like to bring up in relation to the topic?

5. Feel free to give us a call or send an email if you have any further ideas

6. Can you suggest any other developers you think we should talk to?

- Many many thanks 


\subsection{Focus Group Questions}

- Introduce selves, each other. Purpose of group...perspectives.

- What is the timescale of the projects on what you work? Spatial Scale

- What funding sources do you generally use?

- How much does financing set the parameters for what the developer can and cannot do?

- What do you think about the capacity of advisors and consultants to influence what developers do. Perhaps in the context of climate change, but general sustainability.

- The whole idea of the expert professional giving advice to the developer. How realistic is that, and to what extent are they constrained by their company, to make money, etc.

- Do you think climate change is a significant issue for developers and the industry in general?

- Maybe we can talk about the physical impacts, such as increasing cyclones and temperatures, maybe put aside whether you think it is manmade or not; but say in the future, there has been enough of a change... what do you think increasing floods and cyclones, etc. might mean for your business, what changes might you have to make to deal with that? Like changing locations, or markets.

- Do you think the physical risks of climate change are real to developers, that they take notice of these risks; like the risks of flooding?

- Have you seen any evidence of some of these responses?

- Information about climate change; perceptions and attitudes. Understanding of risk.

- Perhaps a bit more about the policy and broader political changes? Regulation etc.?

- Can you comment on the changes and the response to regulation, and who's capable of adapting and who is not? Capacity of developers to respond to changes in regulation?

- What makes a difference between some developers and others, in how long they can hold out when regulatory issues take extensive time? So you need more clarity and certainty of decision-making; that it should not take as long.

- What do you think adaptation to climate change, what do you think it means in the context in what property developers do? What does actual adaptation mean?

- To you ideally, what should a developer do to adapt to climate change?

- One of the assumptions is that it is going to be different not only on the developer, but on the way they might respond and the risks. What it means for different players?

- What about a bit of the learning between developers, when a developer makes some sort of innovation, say doing something a bit more green. Do other developers emulate any of that? 
- Is it coming from the market or from the developers; like the take-up of solar PVs.

- Do the exceptional ones shape what happens in the industry? Are they more likely to do innovations during boom times as opposed to GFC times?

- For those developers who are not innovative, what stops it happening?

- What about the sustainability signal coming from the market for commercial development. How long has this been coming through and what developers are doing this?

- Capacity to make changes, and who is more likely to be in a position to think strategically to protect themselves and deal with some of the changes. Who is more capable of thinking in the longer term about some of the users? Some of the differences in the industry.

- Thinking about capacity building in the development building. How would you go about it? Is there a scope for professionals to be involved to help the development industry adapt.

- Is there something that you would like to bring up, that we haven't thought of 


\subsection{Glossary and Definitions}

Adaptation: Adjustment in natural or human systems in response to actual or expected climatic stimuli or their effects, which moderates harm or exploits beneficial opportunities. Various types of adaptation can be distinguished, including anticipatory, autonomous and planned adaptation:

Anticipatory adaptation: Adaptation that takes place before impacts of climate change are observed. Also referred to as proactive adaptation.

Autonomous adaptation: Adaptation that does not constitute a conscious response to climatic stimuli but is triggered by ecological changes in natural systems and by market or welfare changes in human systems. Also referred to as spontaneous adaptation.

Planned adaptation: Adaptation that is the result of a deliberate policy decision, based on an awareness that conditions have changed or are about to change and that action is required to return to, maintain, or achieve a desired state.

Adaptive capacity (in relation to climate change impacts): The ability of a system to adjust to climate change (including climate variability and extremes) to moderate potential damages, to take advantage of opportunities, or to cope with the consequences

Anthropogenic: Resulting from or produced by human beings.

Climate change: Climate change refers to a change in the state of the climate that can be identified (e.g., by using statistical tests) by changes in the mean and/or the variability of its properties, and that persists for an extended period, typically decades or longer. Climate change may be due to natural internal processes or external forcings, or to persistent anthropogenic changes in the composition of the atmosphere or in land use. Note that the Framework Convention on Climate Change (UNFCCC), in its Article 1, defines climate change as: 'a change of climate which is attributed directly or indirectly to human activity that alters the composition of the global atmosphere and which is in addition to natural climate variability observed over comparable time periods'. The UNFCCC thus makes a distinction between climate change attributable to human activities altering the atmospheric composition, and climate variability attributable to natural causes.

Climate projection: The calculated response of the climate system to emissions or concentration scenarios of greenhouse gases and aerosols, or radiative forcing scenarios, often based on simulations by climate models. Climate projections are distinguished from climate predictions, in that the former critically depend on the emissions/concentration/radiative forcing scenario used, and therefore on highly uncertain assumptions of future socio-economic and technological development.

Extreme weather event: An extreme weather event is an event that is rare at a particular place and time of year. Definitions of rare vary, but an extreme weather event would normally be as rare as or rarer than the 10th or 90th percentile of the observed probability density function. By definition, the characteristics of what is called extreme weather may vary from place to place in an absolute sense. Single extreme events cannot be simply and directly attributed to anthropogenic climate change, as there is always a fi nite chance the event in question might have occurred naturally. When a pattern of extreme weather persists for some time, such as a season, it may be classed as an extreme climate event, especially if it yields an average or total that is itself extreme (e.g., drought or heavy rainfall over a season). 
Greenhouse gas (GHG): Greenhouse gases are those gaseous constituents of the atmosphere, both natural and anthropogenic, that absorb and emit radiation at specific wavelengths within the spectrum of thermal infrared radiation emitted by the Earth's surface, the atmosphere itself, and by clouds. This property causes the greenhouse effect. Water vapour $(\mathrm{H} 2 \mathrm{O})$, carbon dioxide (CO2), nitrous oxide (N2O), methane $(\mathrm{CH} 4)$ and ozone $(\mathrm{O} 3)$ are the primary greenhouse gases in the Earth's atmosphere. Moreover, there are a number of entirely human made greenhouse gases in the atmosphere, such as the halocarbons and other chlorine- and bromine-containing substances, dealt with under the Montreal Protocol. Beside $\mathrm{CO} 2, \mathrm{~N} 2 \mathrm{O}$ and $\mathrm{CH} 4$, the Kyoto Protocol deals with the greenhouse gases sulphur hexafluoride (SF6), hydrofluorocarbons (HFCs) and perfluorocarbons (PFCs).

Mitigation: A human intervention to reduce the sources or enhance the sinks of greenhouse gases.

Path dependency: Path dependency describes how decisions made in the past or present restrict the options available in future. For example, the decision to build an airport in a certain area often does not take account future growth of the urban area (or the risk of future climate change impacting that site).

Sea-level rise: An increase in the mean level of the ocean. Eustatic sea-level rise is a change in global average sea level brought about by an increase in the volume of the world ocean. Relative sea-level rise occurs where there is a local increase in the level of the ocean relative to the land, which might be due to ocean rise and/or land level subsidence. In areas subject to rapid land-level uplift, relative sea level can fall.

Spatial immobility: The fact that infrastructure and the like are fixed in space, and cannot easily or cost-effectively be moved or replaced elsewhere. For example, a major highway that runs along a river prone to flooding is fixed in space, and suitable alternate land may not be readily available.

Spatiotemporal scale: Existing in both a spatial scale (i.e. an urban area or smaller area within, such as a suburb) and time (i.e. the change in urban form over time).

Sustainable development: Development that meets the cultural, social, political and economic needs of the present generation without compromising the ability of future generations to meet their own needs.

Urban heat island (UHI): The relative warmth of a city compared with surrounding rural areas, associated with changes in runoff, the concrete jungle effects on heat retention, changes in surface albedo, changes in pollution and aerosols, and so on.

Vulnerability: Vulnerability is the degree to which a system is susceptible to, and unable to cope with, adverse effects of climate change, including climate variability and extremes. Vulnerability is a function of the character, magnitude, and rate of climate change and variation to which a system is exposed, its sensitivity, and its adaptive capacity. 


\subsection{Acronyms}

ABARE: Australian Bureau of Agricultural and Resource Economics

ABCB: Australian Building Codes Board

AGPC: Australian Government Productivity Commission

AHD: Australian Height Datum

ASBEC: Australian Sustainable Built Environment Council

BoM: Bureau of Meteorology

CCS: Carbon Capture and Storage

CMA: Coastal Management Act

CMD: Coastal Management District

COAG: Council of Australian Governments

CPRS: Carbon pollution Reduction Scheme

CSIRO: Commonwealth Scientific and Industrial Research Organisation

CSR: Corporate Social Responsibility

DCC: Department of Climate Change

DCCEE: Department of Climate Change and Energy Efficiency

DERM: Department of Environment and Resource Management

DLGP: Department of Local Government and Planning

DRO: Desired Regional Outcomes

EIB: European Investment Bank

EMS: Environment Management System

GBCA: Green Building Council of Australia

GDP: Gross Domestic Product

GFC: Global Financial Crisis

GHG: Greenhouse Gases

GST: Goods and Service Tax

IAA: Institute of Actuaries

IAG: Insurance Australia Group

ICA: Insurance Council Australia

IPCC: Intergovernmental Panel on Climate Change

LGA: Local Government Association

MAV: Municipal Association of Victoria

NCCAF: National Climate Change Adaptation Framework

NCCF: National Climate Change Forum

NCCT: National Climate Change Taskforce

NGER: National Greenhouse and Energy Reporting

NHMA: Natural Hazard Management Area

144 The capacities of private developers in urban climate change adaptation 
NSEE: National Strategy for Energy Efficiency

QCP: Queensland Coastal Plan

SEQ: South East Queensland

SEQRP: South East Queensland Regional Plan

SPA: Sustainable Planning Act

SPOLAA: Sustainable Planning and other Legislation Amendment Act SPP: State Planning Policy

SPPF: State Planning Policy Framework

UDIA: Urban Development Institute of Australia

UNDP: United Nations Development Program

UNEP FI: United Nations Environmental Program Finance Initiative

UNEP SBCI: United National Environmental Program Sustainable Buildings and Climate Initiative

UNISDR: United Nations International Strategy for Disaster Reduction

VBRC: Victorian Bushfire Royal Commission

VMT: Vehicles Miles Travelled 


\subsection{Coding for Interviews and Focus Groups}

$\begin{array}{ll}\text { Code } & \text { Interpretation } \\ \text { AR1 } & \text { Architect 1 } \\ \text { AR2 } & \text { Architect 2 } \\ \text { CS1 } & \text { Consultant 1 } \\ \text { CS2 } & \text { Consultant 2 } \\ \text { CS3 } & \text { Consultant 3 } \\ \text { CS4 } & \text { Consultant 4 } \\ \text { CS5 } & \text { Consultant 5 } \\ \text { CS6 } & \text { Consultant 6 } \\ \text { CS7 } & \text { Consultant 7 } \\ \text { CS8 } & \text { Consultant 8 } \\ \text { CS9 } & \text { Consultant 9 } \\ \text { FI1 } & \text { Financier 1 } \\ \text { FI2 } & \text { Financier 2 } \\ \text { IN1 } & \text { Industry Body 1 } \\ \text { LD1 } & \text { Large Developer 1 } \\ \text { LD2 } & \text { Large Developer 2 } \\ \text { LG1 } & \text { Local Government 1 } \\ \text { LW1 } & \text { Lawyer 1 } \\ \text { MD1 } & \text { Medium Developer 1 } \\ \text { MD2 } & \text { Medium Developer 2 } \\ \text { MD3 } & \text { Medium Developer 3 } \\ \text { SC1 } & \text { Sustainability Consultant 1 } \\ \text { SC2 } & \text { Sustainability Consultant 2 } \\ \text { SD1 } & \text { Small Developer 1 } \\ \text { SD2 } & \text { Small Developer 2 } \\ \text { SG1 } & \text { State Government 1 } \\ & \\ \text { Con } 1\end{array}$

146 The capacities of private developers in urban climate change adaptation 


\subsection{Survey Questions}

\section{Property Developers Adapting to Climate Change}

Introduction

Developing Adaptively: The role and capacities of private sector developers and financing in urban climate change adaptation.

Are you a property developer, or employed by a property development firm?

This Questionnaire is specifically aimed at private sector property developers.

The Urban Research Program of Griffith University (with funding and in-kind support from the National Climate Change Adaptation Research Facility (NCCARF), and the Urban Development Institute of Australia (UDIA)), are undertaking a research project to investigate the capacity of the private urban development sector to adapt to climate change. The research team includes: Associate Professor Jago Dodson; Dr Eddo Coiacetto; Ms Zsuzsa Banhalmi-Zakar. Ms Pazit Taygfeld, and Ms Heather Shearer.

By understanding the capacity of the sector to improve its climate adaptation responses, the project will advance the national objective of ensuring Australian cities can weather the effects of climate change.

The outputs of the research will also be used to produce a final project report, and 3 peer reviewed academic publications.

We encourage you to complete this questionnaire and thank you in advance for your time. The questionnaire will take about $10-20$ minutes to complete.

Please note that your participation is completely confidential and that no identifiable information will be used at any time.

By completing this survey you will have the opportunity of winning a $\$ 150$ voucher from Dymocks Book Store. Please see terms and conditions for details.

For more information and/or questions please contact:

Heather Shearer

Senior Research Assistant

Urban Research Program

Griffith University

Phone (07)3735 4803

Email: h. shearer@griffith edu au

*1. If you wish further information on the Terms and Conditions, and the Ethical Conduct of this survey, please select Yes; or select No, to continue to Survey. If you select No, you will be deemed to have consented to your participation in the research.

Yes

No 


\section{Property Developers Adapting to Climate Change}

\section{Ethical Conduct of Survey - Information Sheet}

The ethical conduct of this research This survey has been approved by the Griftith Universily Research Ethics Committee: Approval Number; ENV/54/11/HREC. Griffith University conducts research in accordance with the National Statement on Ethical Conduct in Human Research (2007). If you have any concerns or complaints in relation to the ethical conduct of this survey. please contact the Manager, Research Ethics, Office for Research, Bray Centre Nathan Campus, Griffith University, Tet; 38755585 or EMail.

\section{Participation in this research}

Participants will be members of the urban development industry sector in South East Queensland and Melbourne: and either developers or developer peak body representatives.

Selection of participants will be undertaken in consultation with the Queensland division of the Urban Development Institute of Australia (UDIA).

Participation is voluntary and you are free to withdraw from the research at any time.

We do not anticipate any risks to you as a result of your completing this survey.

\section{Your confidentiality}

Participants will be self-selected by means of a link in the UDIA email newsletter. Your survey responses are confidential, and can only be accessed by the researchers. You are not required to give your name or that of your organisation. It may be possible to infer the identity of some organisations because of information collected, but not the identity of the person completing the survey. No identifying information will be available to anyone outside the research team, who are bound by the Griffith University Ethics policy.

All material collected will be stored in a secure on-line account (Survey Monkey). The results of the research may be used in academic publications and conferences by the researchers, and reports to Industry Partners. No identifying details of the participants or their present or previous employers will be disclosed in any publications

\section{Privacy Statement}

For further information consult the University's Privacy Plan at Griffith University Privacy Plan or telephone (07) 3875 5585.

\section{Feedback to you}

The project findings and reporting will be accessed by users in three key ways: the Project website; Project bulletins: and Hard copy project reports.

\section{Prize Draw}

If you choose to participate in the prize draw. your contact information will only be used for the purpose of the prize draw and will not be associated with your survey responses.

\section{Expressing consent}

If you complete, or partially complete, and submit the questionnaire, you will be deemed to have consented to your participation in the research. You may print and retain this sheet for your later reference. 


\section{Property Developers Adapting to Climate Change}

\section{About Your Company}

To begin with, we would Ike to ask you a couple of questions about your company. The purpose of this is to differentiate between developers; and those in allied industries, such as construction or consulting. With the exception of Question 3; all questions are optional.

\section{Are you (or your firm) a member of the UDIA?}
(res
No
Dont Know

\section{Category of Developer}

DevelcperiBuider $<\$ 10$ milion turnover

ContractormanufactureerSupplieriNon Cont

DevelopeciBuilder \& \$20 milition turnover

Financial Institution

DevelepeciBuider > $\$ 20$ milition turnover

stane Schoel / Local Governmem / Utilly Authority

Consintam

Insstution or Non Prefit

Other (please specify)

4. How many years has your firm been involved in property development?
is
$5-10$
$10-15$
$15-20$
$\mathrm{O}>20$

5. Number of Staff in Development Section of Firm
$0<5$
$5-20$
$20-200$
> $>200$

\section{Average Size of Development Project}
O $<2$ lots or dwellings
$11 \cdot 20$ lots or owellings
3- 5 lots or dwelings
21 - 50 lots or owellings
$6 \cdot 10$ bots or owellings
$>50$ lots or dwellings

7. Is your company listed on the ASX?

Yes

No

8. Is property development the primary business activity of your firm?

res

No

9. Apart from property development (if relevant), in what other sectors does your company operate? 


\section{Property Developers Adapting to Climate Change}

\section{Development Profile}

This page aims to establish what type of development your company practices. As the focus of this research is on adaptation to climate change; the type of development, and the target market, may be relevant in identifying adaptation options, and capacity for adaptation. The final question on this page aims to identify some of the issues that you see as most relevant to your company. As with the previous page, all questions are optional.

\section{Areas in which your firm operates? (Select any that apply)}
International
Australia
New ZealandPacinc Islands
$\square$ victoria
Other Austratan States and Temborion

11. If Queensland, Regions in which your firm operates? (Select any that apply)

$\square$ South East Queensland
$\square$ Dating Downs
$\square$ Wde Bay Bumett
$\square$ South West
$\square$ Contral Wost

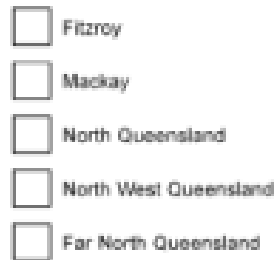

Map of Regions

12. If South East Queensland, what locations does your company usually develop (Select any that apply)
Brisbane CeD
Inner Urban (<5km from CBO)
Inner Suburban (5 - 10km from CBD)

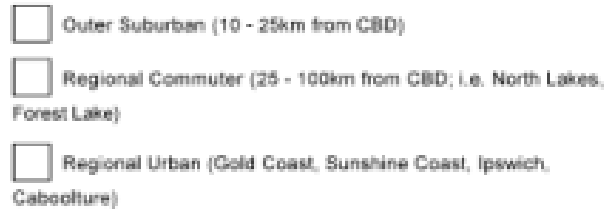

13. If Victoria, areas in which your firm operates? (Select any that apply)
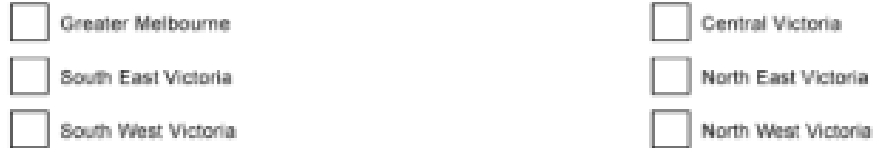

Map of Regions

14. If Greater Melbourne, in what locations does your firm usually develop? (Select any that apply)
$\square$ Mebourne CBD
Outer Suburtan (10 - 2tkm from CBD)
Inner Urban $(<5 \mathrm{~km}$ from $\mathrm{CBO})$
$\square$ Regional Commuter $(25-100 \mathrm{~km}$ from CBD $)$
Inner Suburban (\$ - $10 \mathrm{~km}$ from CBD)
Regional Urban 0.e. Sunbury, Metion, Geelong) 


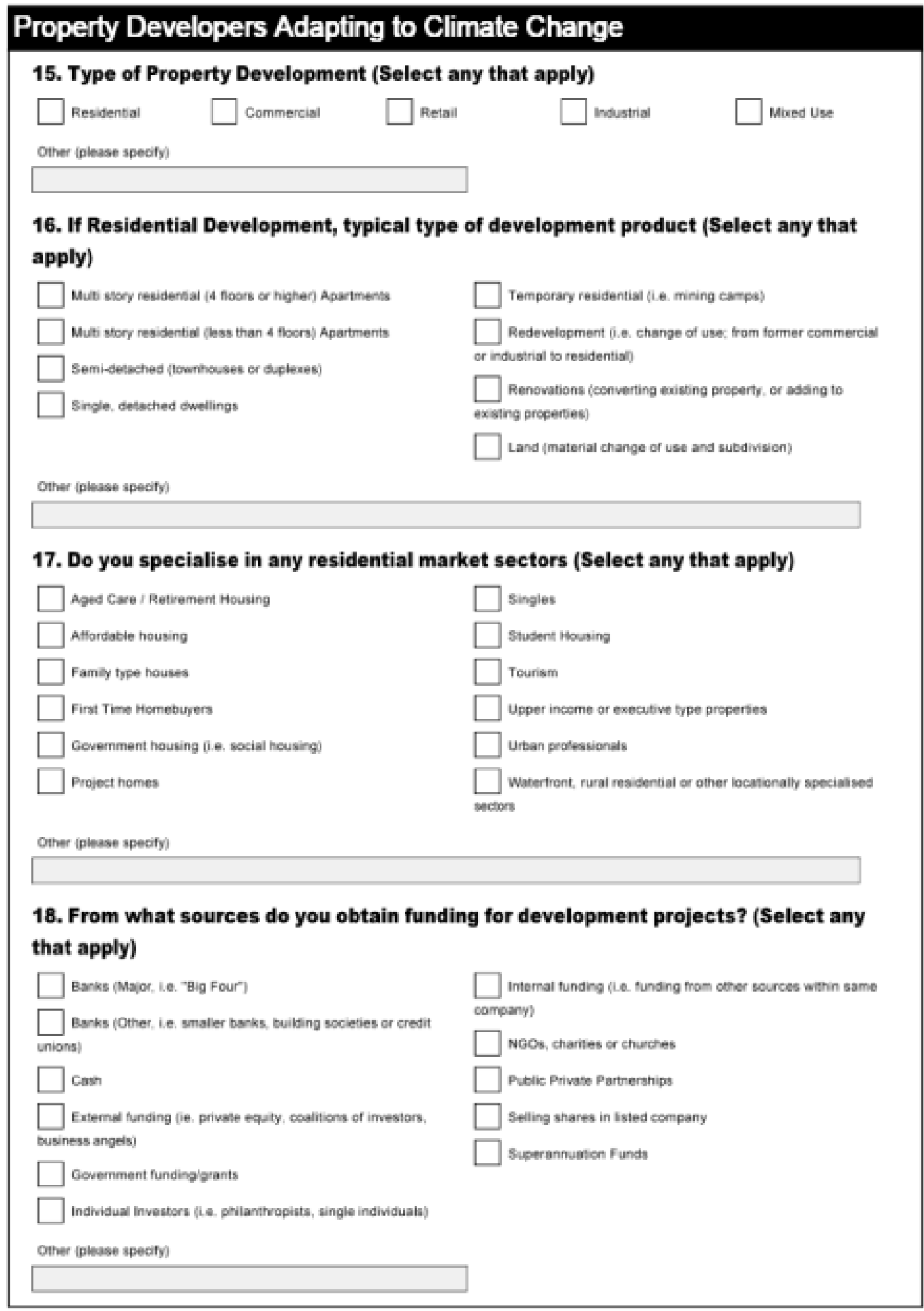




\section{Property Developers Adapting to Climate Change}

19. What issues are of most significance to you as a developer? (Select any that apply)

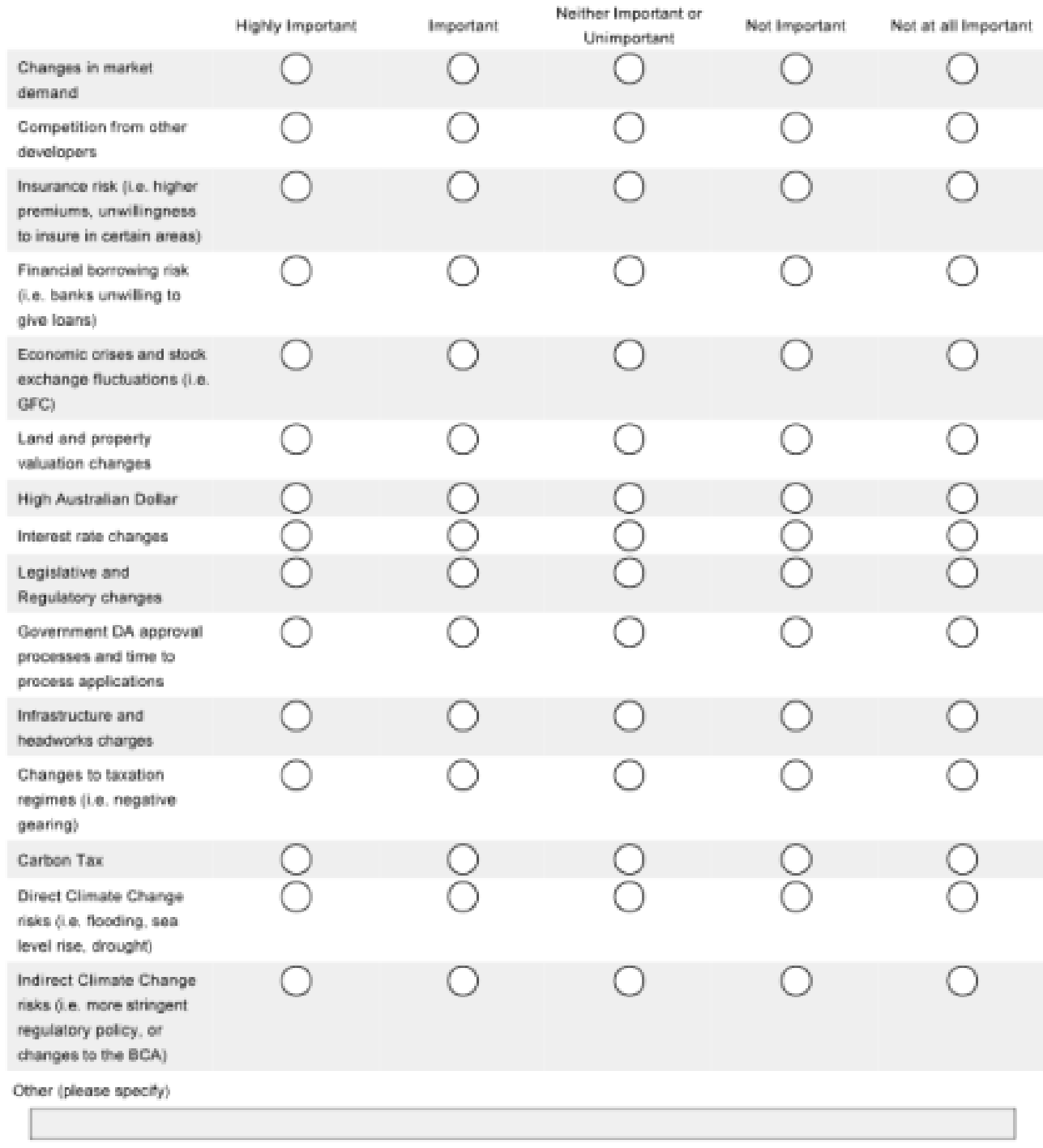

Page 6 


\section{Property Developers Adapting to Climate Change}

\section{Your Firm and Climate Change}

This section is designed to find out some of your firm's responses to climate change.

Climate change is forecast to affect the human environment; in a direct and also indirect manner. Direct (or primary) hazards from climate change include increased incidence of extreme weather events, such as floods or bushfire. Indirect (or secondary and tertiary) hazards are related to regulatory interventions by government or industry such as carbon taxes; and also include legal or insurance issues. Tertiary hazards include societal reactions to change and regulation; such as an increased demand for "greener" properties.

\section{How would you rate your knowledge of climate change?}
$\bigcirc$ Very Good
$($ Peer
Good
verypow
Average

\section{To what extent do you think climate change poses a threat to the development} activities of your firm?
Very high threst
Low threat
High threat
very low threat
Neither high nor low threat

\section{From where do you obtain your information on climate change?}

$\square$ Academic journals
$\square$ Gevemment
$\square$ intemet

Other (plesse specify)

23. How do you rate your knowledge of the following climate change related policy options?

Clean Entrgy Lrgislative
Package (aka Carbon Tax)
National Strategy on
Enorgy Emoiency
National Greenhouse and
Energy Reporting Act




\section{Property Developers Adapting to Climate Change}

24. If you develop in Queensland, how do you rate your knowledge of the following climate change related policy options?

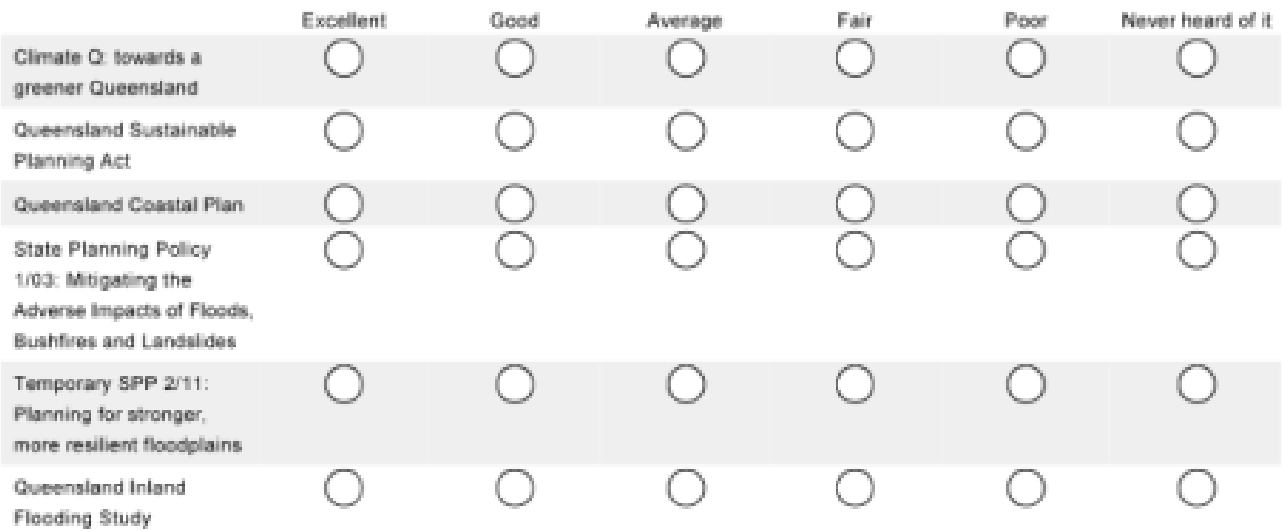

25. If you develop in Victoria, how do you rate your knowledge of the following climate change related policy options?

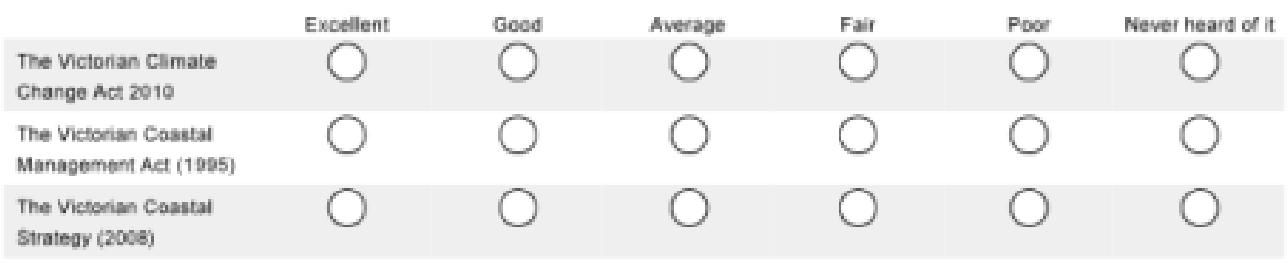

26. To what extent would the following primary hazards of climate change impact the development activity of your firm? (Primary hazards are physical weather or climate impacts, such as individual weather events (hailstorms or cyclones); changes in means (e.g. reduced average rainfall) or shifts in climate-linked systems (e.g. El Nino).

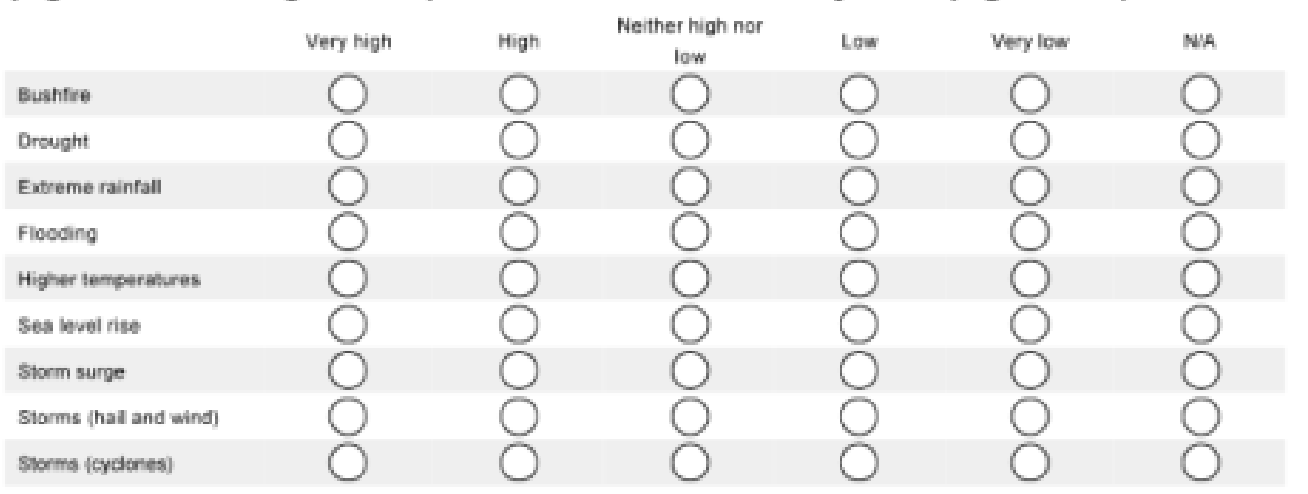

Ceter (pleast specily) 


\section{Property Developers Adapting to Climate Change}

27. To what extent would the following secondary hazards of climate change impact the development activities of your firm? (Note: secondary (or indirect) hazards include regulatory interventions by government or industry to address climate change; e.g. GHG emissions trading schemes, Carbon taxes, or new building standards; and indirect tertiary hazards include societal reactions to change and regulation, such as changes in market demand due to climate change).

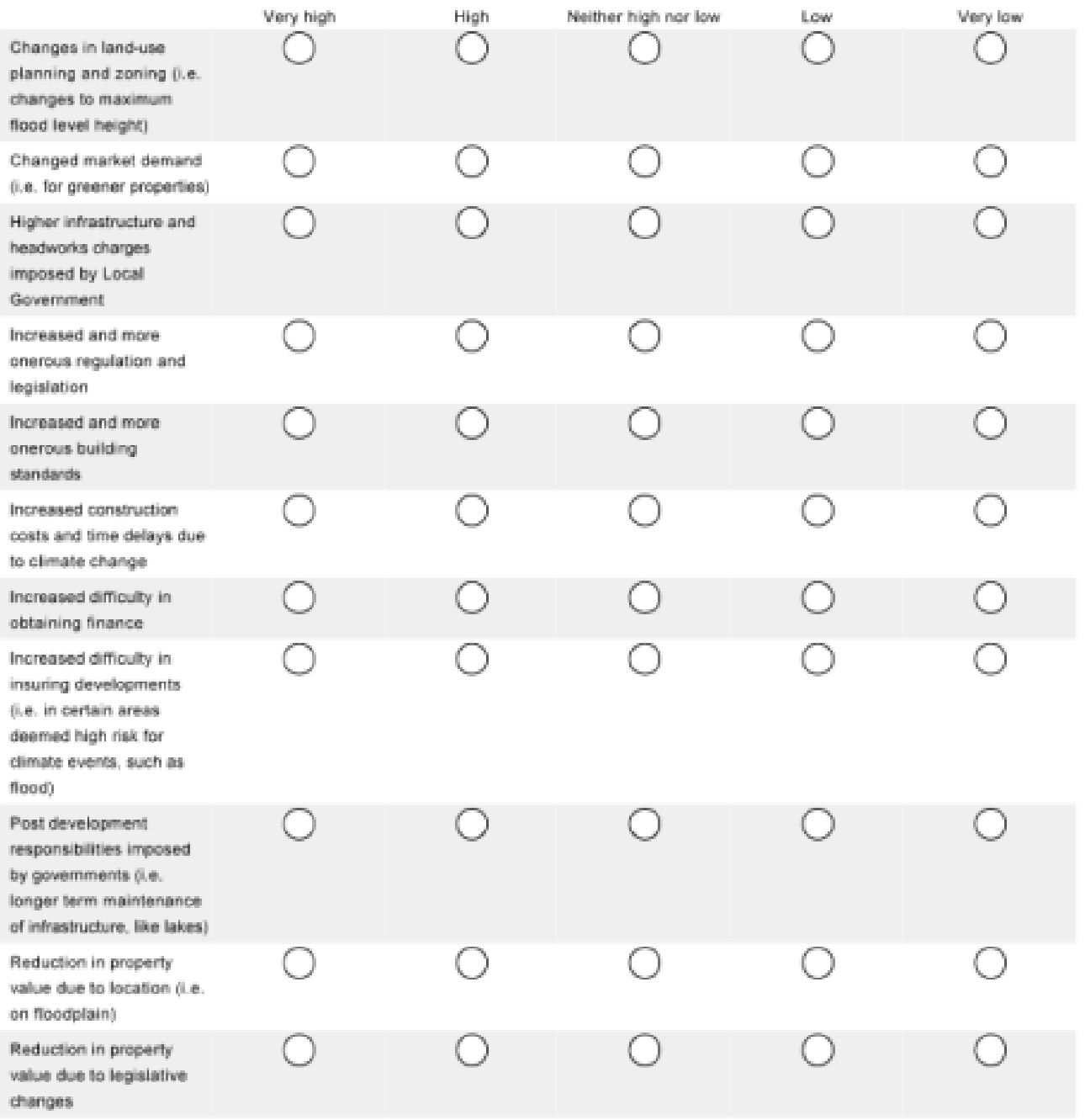




\section{Property Developers Adapting to Climate Change}

\section{Adaptation to Climate Change}

This page is designed to find out some more about whether your firm is currently adapting. or planning to adapt, to climate change.

A company may chose to adapt to climate change; i.e. to change behaviour or ways of doing things to lessen the impacts when they occur.

Adaptation may be defined as "Adjustment in ecological, social, or economic systems in response to actual or expected climatic stimuli, and their effects or impacts" (IPCC), 1995).

The page also seeks to understand what you see as some of the drivers that would encourage your fim to implement adaptive measures, and some barriers to adaptation.

28. Does your firm incorporate adaptation to future climate change into decision making or current operations?
Yes
No
Maybe
Donit know

29. If yes, how?

$\square$ Long term planning

sine location

Clmate resilent infrastructure

Changed layout of lots

Financial modelling

Other (please specify) 


\section{Property Developers Adapting to Climate Change}

30. How does your firm see its role in adapting to climate change? Please say whether you agree or disagree with each statement.

It is Covernmerts:

responsibility to adapt to

climate change fi.e. by

providing improved

intrastructure, such as ses

walls,

Companiss should be

compensated for cosss of

adaptason, such as loss in

land value. or being

forced to provide more

expensive infrastructure

Developers should be

proactive and introduce

their own standards and

certifications to aid

adaptation to climate

change

Devetopers should not be

held responsible for

climate change imgacts

on developments aties

sale

Govemment needs to

conaull more with

develepers, particularty if

interodueing new tares of

regulation

Adapting to climate

change now will have

future benefits

Developera need more

time to adjust their

business to accommodate

to changes in povernment

legislation and regulation

Developers are unfairty

blamed for emvironmental

iscues
Strongly Agree

(

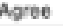

0

Neutral<smiles>O</smiles><smiles>[CH]1[CH]C1</smiles>

Strongly Disagree

0

0

0

0

0

0

0

0

O

0

0

0
0

0

0

0
0

0

O

0

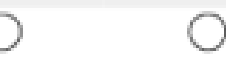

O<smiles></smiles>

0

O

$\mathrm{O}$

0

0 


\section{Property Developers Adapting to Climate Change}

31. Which of the following would make your firm more likely to implement climate change adaptation measures?

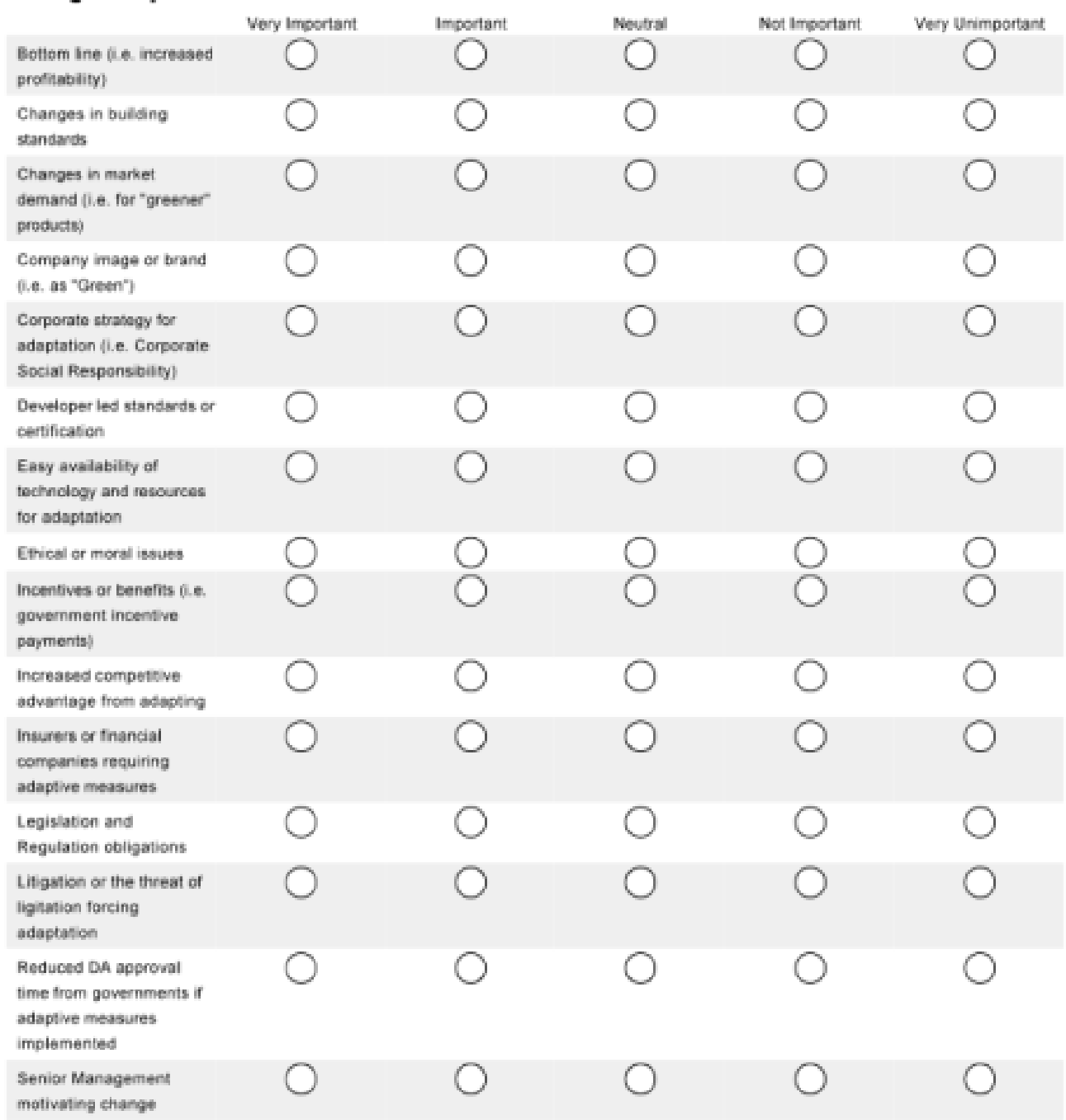

32. Do you use any of the following Industry Tools, Certification and Standards

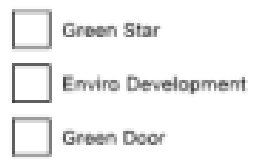

$\square$ Corporase Social Responsibility

ther (please specity) 


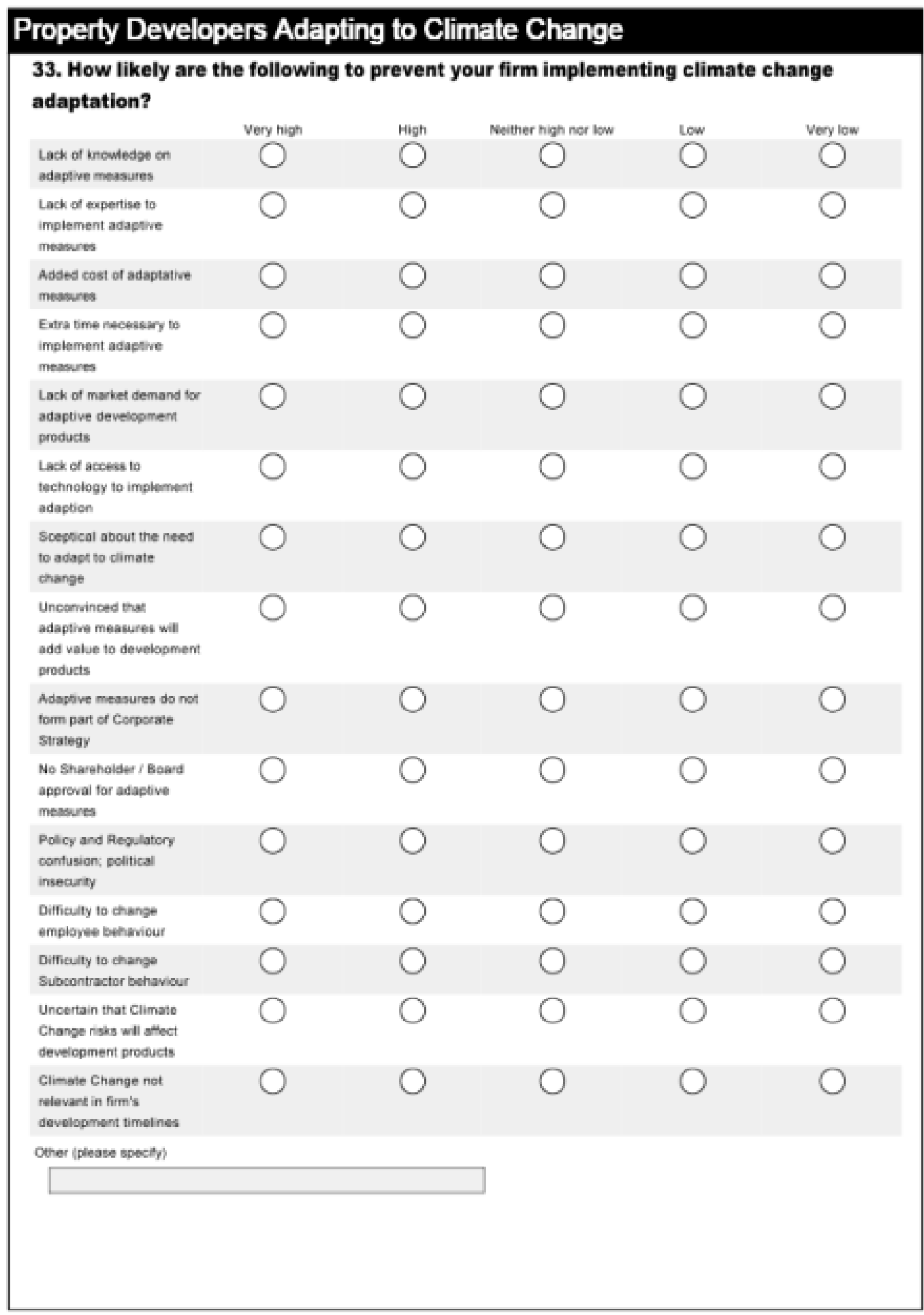

Page 13 


\section{Property Developers Adapting to Climate Change}

34. Do you have any further comments?

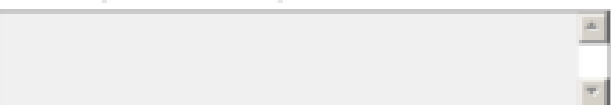

35. Thank you very much for taking the time to help us with our research. Finally, would you like to be involved in further research; i.e. attend a short focus group, and or enter our draw to win a $\mathbf{\$ 1 5 0}$ book voucher?

Participase in Further Research

Enter the Prize Drase

Neither 


\section{Property Developers Adapting to Climate Change}

\section{Prize Draw}

Please fill in the following information. All such details are confidential, will not be associated with your survey responses, and such information will be permanently deleted after drawing the prize. All prize winners will be notified by email by no later than 31 August 2012.

36. Do you want to participate in further research, enter the prize draw, or both?

Prize Draw

Further Research

37. If you wish to enter our Prize Draw or Participate in Further Research, or both; please fill in your name, email address and telephone number.

Name:

Email Address:

Phone Number 




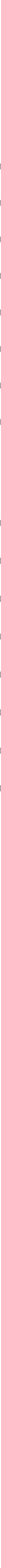

